\title{
Nonlinear Expectations and Stochastic Calculus under Uncertainty
}

—with Robust Central Limit Theorem and G-Brownian Motion

\author{
Shige PENG \\ Institute of Mathematics \\ Shandong University \\ 250100, Jinan, China \\ peng@sdu.edu.cn
}

Version: first edition 


\section{Preface}

This book is focused on the recent developments on problems of probability model under uncertainty by using the notion of nonlinear expectations and, in particular, sublinear expectations. Roughly speaking, a nonlinear expectation $\mathbb{E}$ is a monotone and constant preserving functional defined on a linear space of random variables. We are particularly interested in sublinear expectations, i.e., $\mathbb{E}[X+Y] \leq \mathbb{E}[X]+\mathbb{E}[Y]$ for all random variables $X, Y$ and $\mathbb{E}[\lambda X]=\lambda \mathbb{E}[X]$ if $\lambda \geq 0$.

A sublinear expectation $\mathbb{E}$ can be represented as the upper expectation of a subset of linear expectations $\left\{E_{\theta}: \theta \in \Theta\right\}$, i.e., $\mathbb{E}[X]=\sup _{\theta \in \Theta} E_{\theta}[X]$. In most cases, this subset is often treated as an uncertain model of probabilities $\left\{P_{\theta}: \theta \in \Theta\right\}$ and the notion of sublinear expectation provides a robust way to measure a risk loss $X$. In fact, the sublinear expectation theory provides many rich, flexible and elegant tools.

A remarkable point of view is that we emphasize the term "expectation" rather than the well-accepted classical notion "probability" and its non-additive counterpart "capacity". A technical reason is that in general the information contained in a nonlinear expectation $\mathbb{E}$ will be lost if one consider only its corresponding "non-additive probability" or "capacity" $\mathbb{P}(A)=\mathbb{E}\left[\mathbf{1}_{A}\right]$. Philosophically, the notion of expectation has its direct meaning of "mean", "average" which is not necessary to be derived from the corresponding "relative frequency" which is the origin of the probability measure. For example, when a person gets a sample $\left\{x_{1}, \cdots, x_{N}\right\}$ from a random variable $X$, he can directly use $\bar{X}=\frac{1}{N} \sum x_{i}$ to calculate its mean. In general he uses $\overline{\varphi(X)}=\frac{1}{N} \sum \varphi\left(x_{i}\right)$ for the mean of $\varphi(X)$. We will discuss in detail this issue after the overview of our new law of large numbers (LLN) and central limit theorem (CLT).

A theoretical foundation of the above expectation framework is our new LLN and CLT under sublinear expectations. Classical LLN and CLT have been widely used in probability theory, statistics, data analysis as well as in many practical situations such as financial pricing and risk management. They provide a strong and convincing way to explain why in practice normal distributions are so widely utilized. But often a serious problem is that, in general, the "i.i.d." condition is difficult to be satisfied. In practice, for the most real-time processes and data for which the classical trials and samplings become impossible, the uncertainty of probabilities and distributions can not be neglected. In fact the abuse of normal distributions in finance and many other industrial or commercial 
domains has been criticized.

Our new CLT does not need this strong "i.i.d." assumption. Instead of fixing a probability measure $P$, we introduce an uncertain subset of probability measures $\left\{P_{\theta}: \theta \in \Theta\right\}$ and consider the corresponding sublinear expectation $\mathbb{E}[X]=\sup _{\theta \in \Theta} E_{\theta}[X]$. Our main assumptions are:

(i) The distribution of $X_{i}$ is within a subset of distributions $\left\{F_{\theta}(x): \theta \in \Theta\right\}$ with

$$
\bar{\mu}=\mathbb{E}\left[X_{i}\right] \geq \underline{\mu}=-\mathbb{E}\left[-X_{i}\right]
$$

(ii) Any realization of $X_{1}, \cdots, X_{n}$ does not change the distributional uncertainty of $X_{n+1}$.

Under $\mathbb{E}$, we call $X_{1}, X_{2}, \cdots$ to be identically distributed if condition (i) is satisfied, and we call $X_{n+1}$ is independent from $X_{1}, \cdots, X_{n}$ if condition (ii) is fulfilled. Mainly under the above weak "i.i.d." assumptions, we have proved that for each continuous function $\varphi$ with linear growth we have the following LLN:

$$
\lim _{n \rightarrow \infty} \mathbb{E}\left[\varphi\left(\frac{S_{n}}{n}\right)\right]=\sup _{\underline{\mu} \leq v \leq \bar{\mu}} \varphi(v) .
$$

Namely, the uncertain subset of the distributions of $S_{n} / n$ is approximately a subset of dirac measures $\left\{\delta_{v}: \mu \leq v \leq \bar{\mu}\right\}$.

In particular, if $\mu=\bar{\mu}=0$, then $S_{n} / n$ converges in law to 0 . In this case, if we assume furthermore that $\bar{\sigma}^{2}=\mathbb{E}\left[X_{i}^{2}\right]$ and $\underline{\sigma}^{2}=-\mathbb{E}\left[-X_{i}^{2}\right], i=1,2, \cdots$, then we have the following generalization of the CLT:

$$
\lim _{n \rightarrow \infty} \mathbb{E}\left[\varphi\left(S_{n} / \sqrt{n}\right)\right]=\mathbb{E}[\varphi(X)] .
$$

Here $X$ is called $G$-normal distributed and denoted by $N\left(\{0\} \times\left[\underline{\sigma}^{2}, \bar{\sigma}^{2}\right]\right)$. The value $\mathbb{E}[\varphi(X)]$ can be calculated by defining $u(t, x):=\mathbb{E}[\varphi(x+\sqrt{t} X)]$ which solves the partial differential equation (PDE) $\partial_{t} u=G\left(u_{x x}\right)$ with $G(a):=$ $\frac{1}{2}\left(\bar{\sigma}^{2} a^{+}-\underline{\sigma}^{2} a^{-}\right)$. Our results reveal a deep and essential relation between the theory of probability and statistics under uncertainty and second order fully nonlinear parabolic equations (HJB equations). We have two interesting situations: when $\varphi$ is a convex function, then

$$
\mathbb{E}[\varphi(X)]=\frac{1}{\sqrt{2 \pi \bar{\sigma}^{2}}} \int_{-\infty}^{\infty} \varphi(x) \exp \left(-\frac{x^{2}}{2 \bar{\sigma}^{2}}\right) d x
$$

but if $\varphi$ is a concave function, the above $\bar{\sigma}^{2}$ must be replaced by $\underline{\sigma}^{2}$. If $\underline{\sigma}=\bar{\sigma}=\sigma$, then $N\left(\{0\} \times\left[\underline{\sigma}^{2}, \bar{\sigma}^{2}\right]\right)=N\left(0, \sigma^{2}\right)$ which is a classical normal distribution.

This result provides a new way to explain a well-known puzzle: many practitioners, e.g., traders and risk officials in financial markets can widely use normal distributions without serious data analysis or even with data inconsistence. In many typical situations $\mathbb{E}[\varphi(X)]$ can be calculated by using normal distributions with careful choice of parameters, but it is also a high risk calculation if the reasoning behind has not been understood. 
We call $N\left(\{0\} \times\left[\underline{\sigma}^{2}, \bar{\sigma}^{2}\right]\right)$ the $G$-normal distribution. This new type of sublinear distributions was first introduced in Peng (2006) 100 (see also 102], 103], [104], 105]) for a new type of " $G$-Brownian motion" and the related calculus of Itô's type. The main motivations were uncertainties in statistics, measures of risk and superhedging in finance (see El Karoui, Peng and Quenez (1997) [44], Artzner, Delbaen, Eber and Heath (1999) [3], Chen and Epstein (2002) [19], Föllmer and Schied (2004) [51]). Fully nonlinear super-hedging is also a possible domain of applications (see Avellaneda, Levy and Paras (1995) [5], Lyons (1995) 82], see also Cheridito, Soner, Touzi and Victoir (2007) 23] where a new BSDE approach was introduced).

Technically we introduce a new method to prove our CLT on a sublinear expectation space. This proof is short since we have borrowed a deep interior estimate of fully nonlinear partial differential equation (PDE) in Krylov (1987) [76]. In fact the theory of fully nonlinear parabolic PDE plays an essential role in deriving our new results of LLN and CLT. In the classical situation the corresponding PDE becomes a heat equation which is often hidden behind its heat kernel, i.e., the normal distribution. In this book we use the powerful notion of viscosity solutions for our nonlinear PDE initially introduced by Crandall and Lions (1983) [29]. This notion is specially useful when the equation is degenerate. For reader's convenience, we provide an introductory chapter in Appendix C. If readers are only interested in the classical non-degenerate cases, the corresponding solutions will become smooth (see the last section of Appendix C).

We define a sublinear expectation on the space of continuous paths from $\mathbb{R}^{+}$ to $\mathbb{R}^{d}$ which is an analogue of Wiener's law, by which a $G$-Brownian motion is formulated. Briefly speaking, a $G$-Brownian motion $\left(B_{t}\right)_{t \geq 0}$ is a continuous process with independent and stationary increments under a given sublinear expectation $\mathbb{E}$.

$G$-Brownian motion has a very rich and interesting new structure which nontrivially generalizes the classical one. We can establish the related stochastic calculus, especially Itô's integrals and the related quadratic variation process $\langle B\rangle$. A very interesting new phenomenon of our $G$-Brownian motion is that its quadratic variation process $\langle B\rangle$ is also a continuous process with independent and stationary increments, and thus can be still regarded as a Brownian motion. The corresponding G-Itô's formula is obtained. We have also established the existence and uniqueness of solutions to stochastic differential equation under our stochastic calculus by the same Picard iterations as in the classical situation.

New norms were introduced in the notion of $G$-expectation by which the corresponding stochastic calculus becomes significantly more flexible and powerful. Many interesting, attractive and challenging problems are also automatically provided within this new framework.

In this book we adopt a novel method to present our $G$-Brownian motion theory. In the first two chapters as well as the first two sections of Chapter III, our sublinear expectations are only assumed to be finitely sub-additive, instead of " $\sigma$-sub-additive". This is just because all the related results obtained in this part do not need the " $\sigma$-sub-additive" assumption, and readers even need not to 
have the background of classical probability theory. In fact, in the whole part of the first five chapters we only use a very basic knowledge of functional analysis such as Hahn-Banach Theorem (see Appendix A). A special situation is when all the sublinear expectations in this book become linear. In this case this book can be still considered as using a new and very simple approach to teach the classical Itô's stochastic calculus, since this book does not need the knowledge of probability theory. This is an important advantage to use expectation as our basic notion.

The "authentic probabilistic parts", i.e., the pathwise analysis of our $G$ Brownian motion and the corresponding random variables, view as functions of $G$-Brownian path, is presented in Chapter VI. Here just as the classical " $P$-sure analysis", we introduce "ĉ-sure analysis" for $G$-capacity ĉ. Readers who are not interested in the deep parts of stochastic analysis of $G$-Brownian motion theory do not need to read this chapter.

This book was based on the author's Lecture Notes [102] for several series of lectures, for the 2nd Workshop Stochastic Equations and Related Topic Jena, July 23-29, 2006; Graduate Courses of Yantai Summer School in Finance, Yantai University, July 06-21, 2007; Graduate Courses of Wuhan Summer School, July 24-26, 2007; Mini-Course of Institute of Applied Mathematics, AMSS, April 16-18, 2007; Mini-course in Fudan University, May 2007 and August 2009; Graduate Courses of CSFI, Osaka University, May 15-June 13, 2007; Minerva Research Foundation Lectures of Columbia University in Fall of 2008; MiniWorkshop of $G$-Brownian motion and $G$-expectations in Weihai, July 2009, a series talks in Department of Applied Mathematics, Hong Kong Polytechnic University, November-December, 2009 and an intensive course in WCU Center for Financial Engineering, Ajou University. The hospitalities and encouragements of the above institutions and the enthusiasm of the audiences are the main engine to realize this lecture notes. I thank for many comments and suggestions given during those courses, especially to Li Juan and Hu Mingshang. During the preparation of this book, a special reading group was organized with members Hu Mingshang, Li Xinpeng, Xu Xiaoming, Lin Yiqing, Su Chen, Wang Falei and Yin Yue. They proposed very helpful suggestions for the revision of the book. Hu Mingshang and Li Xinpeng have made a great effort for the final edition. Their efforts are decisively important to the realization of this book. 


\section{Contents}

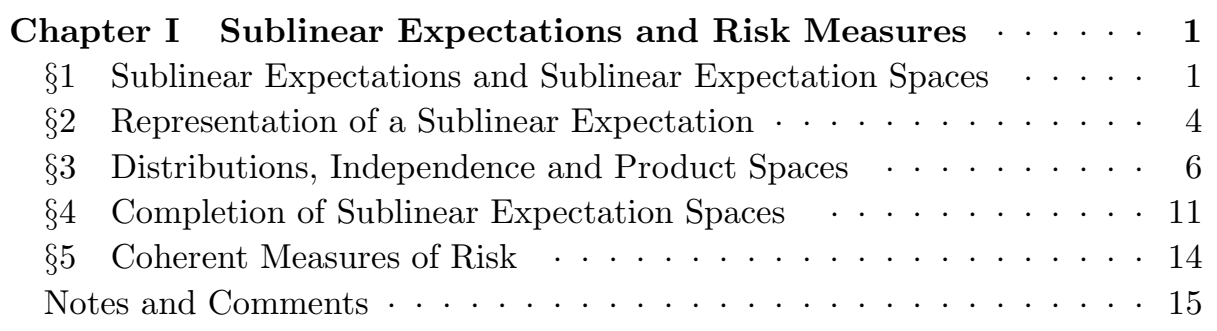

Chapter II Law of Large Numbers and Central Limit Theorem $\mathbf{1 7}$

$\S 1$ Maximal Distribution and $G$-normal Distribution $\ldots \ldots \ldots . . .17$

$\S 2$ Existence of $G$-distributed Random Variables . . . . . . . . . . 24

$\S 3$ Law of Large Numbers and Central Limit Theorem . . . . . . . . 25

Notes and Comments . . . . . . . . . . . . . . 33

Chapter III $G$-Brownian Motion and Itô's Integral $\ldots \ldots \ldots$

$\S 1 \quad G$-Brownian Motion and its Characterization . . . . . . 35

$\S 2$ Existence of $G$-Brownian Motion . . . . . . . . . . . 38

$\S 3$ Itô's Integral with $G$-Brownian Motion $\ldots \ldots \ldots \ldots . . . .42$

$\S 4$ Quadratic Variation Process of $G$-Brownian Motion $\ldots . . . .46$

$\S 5$ The Distribution of $\langle B\rangle \ldots \ldots \ldots \ldots \ldots \ldots \ldots \ldots$

$\S 6 \quad G$-Itô's Formula $\ldots \ldots \ldots \ldots \ldots \ldots$

$\S 7$ Generalized $G$-Brownian Motion $\ldots \ldots \ldots \ldots$

$\S 8 \quad \widetilde{G}$-Brownian Motion under a Nonlinear Expectation . . . . . . . . 64

$\S 9$ Construction of $\widetilde{G}$-Brownian Motions under Nonlinear Expectation 66

Notes and Comments . . . . . . . . . . . . . . . 69

Chapter IV $G$-martingales and Jensen's Inequality $\ldots \ldots \ldots 71$

$\S 1$ The Notion of $G$-martingales $\ldots \ldots \ldots \ldots . \ldots . \ldots 71$

$\S 2 \quad$ On $G$-martingale Representation Theorem . . . . . . . . . . . . 73

$\S 3 \quad G$-convexity and Jensen's Inequality for $G$-expectations $\ldots \ldots$

Notes and Comments . . . . . . . . . . . . . . . . . . 79 
Chapter V Stochastic Differential Equations . . . . . . . 81

$\S 1$ Stochastic Differential Equations . . . . . . . . . . . . . . . 81

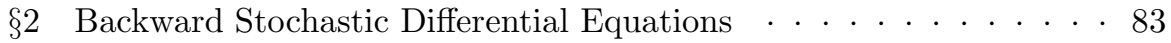

$\S 3$ Nonlinear Feynman-Kac Formula . . . . . . . . . . . . . 85

Notes and Comments . . . . . . . . . . . . . . . . 88

\section{Chapter VI Capacity and Quasi-Surely Analysis for $G$-Brownian}

Paths . . . . . . . . . . . . . . . . . . . 91

$\S 1$ Integration Theory associated to an Upper Probability . . . . . . 91

$\S 2 \quad G$-expectation as an Upper Expectation . . . . . . . . . . . 101

$\S 3 \quad G$-capacity and Paths of $G$-Brownian Motion $\ldots \ldots \ldots$

Notes and Comments . . . . . . . . . . . . . . . . . 106

Appendix A Preliminaries in Functional Analysis $\ldots \ldots \ldots$

$\S 1$ Completion of Normed Linear Spaces . . . . . . . . . . . 107

$\S 2$ The Hahn-Banach Extension Theorem … . . . . . . . . 108

$\S 3$ Dini's Theorem and Tietze's Extension Theorem . . . . . . . 108

Appendix B Preliminaries in Probability Theory . . . . . . . . . 109

$\S 1$ Kolmogorov's Extension Theorem . . . . . . . . . . . . . . . 109

$\S 2$ Kolmogorov's Criterion . . . . . . . . . . . . . . . . . . . . . 1110

$\S 3 \quad$ Daniell-Stone Theorem $\ldots \ldots \ldots 112$

Appendix C Solutions of Parabolic Partial Differential Equation 113

$\S 1$ The Definition of Viscosity Solutions . . . . . . . . . . . . . . . . . . . . . .

$\S 2$ Comparison Theorem . . . . . . . . . . . . . . . . . . 115

$\S 3$ Perron's Method and Existence . . . . . . . . . . . . . 122

$\S 4$ Krylov's Regularity Estimate for Parabolic PDE . . . . . . . 125

Bibliography $\ldots \ldots \ldots \ldots \ldots$

Index of Symbols . . . . . . . . . . . . . . . . . . . . . . . . . 139

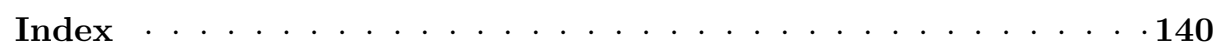




\section{Chapter I}

\section{Sublinear Expectations and Risk Measures}

The sublinear expectation is also called the upper expectation or the upper prevision, and this notion is used in situations when the probability models have uncertainty. In this chapter, we present the basic notion of sublinear expectations and the corresponding sublinear expectation spaces. We give the representation theorem of a sublinear expectation and the notions of distributions and independence under the framework of sublinear expectations. We also introduce a natural Banach norm of a sublinear expectation in order to get the completion of a sublinear expectation space which is a Banach space. As a fundamentally important example, we introduce the notion of coherent risk measures in finance. A large part of notions and results in this chapter will be throughout this book.

\section{$\S 1$ Sublinear Expectations and Sublinear Expec- tation Spaces}

Let $\Omega$ be a given set and let $\mathcal{H}$ be a linear space of real valued functions defined on $\Omega$. In this book, we suppose that $\mathcal{H}$ satisfies $c \in \mathcal{H}$ for each constant $c$ and $|X| \in \mathcal{H}$ if $X \in \mathcal{H}$. The space $\mathcal{H}$ can be considered as the space of random variables.

Definition 1.1 A Sublinear expectation $\mathbb{E}$ is a functional $\mathbb{E}: \mathcal{H} \rightarrow \mathbb{R}$ satisfying

(i) Monotonicity:

$$
\mathbb{E}[X] \geq \mathbb{E}[Y] \text { if } X \geq Y \text {. }
$$

(ii) Constant preserving:

$$
\mathbb{E}[c]=c \quad \text { for } c \in \mathbb{R} .
$$


(iii) Sub-additivity: For each $X, Y \in \mathcal{H}$,

$$
\mathbb{E}[X+Y] \leq \mathbb{E}[X]+\mathbb{E}[Y] .
$$

(iv) Positive homogeneity:

$$
\mathbb{E}[\lambda X]=\lambda \mathbb{E}[X] \quad \text { for } \lambda \geq 0 .
$$

The triple $(\Omega, \mathcal{H}, \mathbb{E})$ is called a sublinear expectation space. If (i) and (ii) are satisfied, $\mathbb{E}$ is called a nonlinear expectation and the triple $(\Omega, \mathcal{H}, \mathbb{E})$ is called a nonlinear expectation space.

Definition 1.2 Let $\mathbb{E}_{1}$ and $\mathbb{E}_{2}$ be two nonlinear expectations defined on $(\Omega, \mathcal{H})$. $\mathbb{E}_{1}$ is said to be dominated by $\mathbb{E}_{2}$ if

$$
\mathbb{E}_{1}[X]-\mathbb{E}_{1}[Y] \leq \mathbb{E}_{2}[X-Y] \quad \text { for } X, Y \in \mathcal{H} .
$$

Remark 1.3 From (iii), a sublinear expectation is dominated by itself. In many situations, (iii) is also called the property of self-domination. If the inequality in (iii) becomes equality, then $\mathbb{E}$ is a linear expectation, i.e., $\mathbb{E}$ is a linear functional satisfying (i) and (ii).

Remark 1.4 (iii)+(iv) is called sublinearity. This sublinearity implies (v) Convexity:

$$
\mathbb{E}[\alpha X+(1-\alpha) Y] \leq \alpha \mathbb{E}[X]+(1-\alpha) \mathbb{E}[Y] \text { for } \alpha \in[0,1] .
$$

If a nonlinear expectation $\mathbb{E}$ satisfies convexity, we call it a convex expectation.

The properties (ii)+(iii) implies

(vi) Cash translatability:

$$
\mathbb{E}[X+c]=\mathbb{E}[X]+c \text { for } c \in \mathbb{R} .
$$

In fact, we have

$$
\mathbb{E}[X]+c=\mathbb{E}[X]-\mathbb{E}[-c] \leq \mathbb{E}[X+c] \leq \mathbb{E}[X]+\mathbb{E}[c]=\mathbb{E}[X]+c .
$$

For property (iv), an equivalence form is

$$
\mathbb{E}[\lambda X]=\lambda^{+} \mathbb{E}[X]+\lambda^{-} \mathbb{E}[-X] \text { for } \lambda \in \mathbb{R} .
$$

In this book, we will systematically study the sublinear expectation spaces. In the following chapters, unless otherwise stated, we consider the following sublinear expectation space $(\Omega, \mathcal{H}, \mathbb{E})$ : if $X_{1}, \cdots, X_{n} \in \mathcal{H}$ then $\varphi\left(X_{1}, \cdots, X_{n}\right) \in$ $\mathcal{H}$ for each $\varphi \in C_{\text {l.Lip }}\left(\mathbb{R}^{n}\right)$ where $C_{\text {l.Lip }}\left(\mathbb{R}^{n}\right)$ denotes the linear space of functions $\varphi$ satisfying

$$
\begin{gathered}
|\varphi(x)-\varphi(y)| \leq C\left(1+|x|^{m}+|y|^{m}\right)|x-y| \text { for } x, y \in \mathbb{R}^{n}, \\
\text { some } C>0, m \in \mathbb{N} \text { depending on } \varphi .
\end{gathered}
$$

In this case $X=\left(X_{1}, \cdots, X_{n}\right)$ is called an $n$-dimensional random vector, denoted by $X \in \mathcal{H}^{n}$. 
Remark 1.5 It is clear that if $X \in \mathcal{H}$ then $|X|, X^{m} \in \mathcal{H}$. More generally, $\varphi(X) \psi(Y) \in \mathcal{H}$ if $X, Y \in \mathcal{H}$ and $\varphi, \psi \in C_{l . L i p}(\mathbb{R})$. In particular, if $X \in \mathcal{H}$ then $\mathbb{E}\left[|X|^{n}\right]<\infty$ for each $n \in \mathbb{N}$.

Here we use $C_{\text {l.Lip }}\left(\mathbb{R}^{n}\right)$ in our framework only for some convenience of techniques. In fact our essential requirement is that $\mathcal{H}$ contains all constants and, moreover, $X \in \mathcal{H}$ implies $|X| \in \mathcal{H}$. In general, $C_{\text {l.Lip }}\left(\mathbb{R}^{n}\right)$ can be replaced by any one of the following spaces of functions defined on $\mathbb{R}^{n}$.

- $\mathbb{L}^{\infty}\left(\mathbb{R}^{n}\right)$ : the space of bounded Borel-measurable functions;

- $C_{b}\left(\mathbb{R}^{n}\right)$ : the space of bounded and continuous functions;

- $C_{b}^{k}\left(\mathbb{R}^{n}\right)$ : the space of bounded and $k$-time continuously differentiable functions with bounded derivatives of all orders less than or equal to $k$;

- $C_{\text {unif }}\left(\mathbb{R}^{n}\right)$ : the space of bounded and uniformly continuous functions;

- $C_{b . L i p}\left(\mathbb{R}^{n}\right)$ : the space of bounded and Lipschitz continuous functions;

- $L^{0}\left(\mathbb{R}^{n}\right)$ : the space of Borel measurable functions.

Next we give two examples of sublinear expectations.

Example 1.6 In a game we select a ball from a box containing $W$ white, $B$ black and $Y$ yellow balls. The owner of the box, who is the banker of the game, does not tell us the exact numbers of $W, B$ and $Y$. He or she only informs us that $W+B+Y=100$ and $W=B \in[20,25]$. Let $\xi$ be a random variable defined by

$$
\xi=\left\{\begin{aligned}
1 & \text { if we get a white ball; } \\
0 & \text { if we get a yellow ball; } \\
-1 & \text { if we get a black ball }
\end{aligned}\right.
$$

Problem: how to measure a loss $X=\varphi(\xi)$ for a given function $\varphi$ on $\mathbb{R}$.

We know that the distribution of $\xi$ is

$$
\left\{\begin{array}{ccc}
-1 & 0 & 1 \\
\frac{p}{2} & 1-p & \frac{p}{2}
\end{array}\right\} \text { with uncertainty: } p \in[\underline{\mu}, \bar{\mu}]=[0.4,0.5]
$$

Thus the robust expectation of $X=\varphi(\xi)$ is

$$
\begin{aligned}
\mathbb{E}[\varphi(\xi)] & :=\sup _{P \in \mathcal{P}} E_{P}[\varphi(\xi)] \\
& =\sup _{p \in[\underline{\mu}, \bar{\mu}]}\left[\frac{p}{2}(\varphi(1)+\varphi(-1))+(1-p) \varphi(0)\right] .
\end{aligned}
$$

Here, $\xi$ has distribution uncertainty. 
Example 1.7 A more general situation is that the banker of a game can choose among a set of distributions $\{F(\theta, A)\}_{A \in \mathcal{B}(\mathbb{R}), \theta \in \Theta}$ of a random variable $\xi$. In this situation the robust expectation of a risk position $\varphi(\xi)$ for some $\varphi \in C_{\text {l.Lip }}(\mathbb{R})$ is

$$
\mathbb{E}[\varphi(\xi)]:=\sup _{\theta \in \Theta} \int_{\mathbb{R}} \varphi(x) F(\theta, d x) .
$$

Exercise 1.8 Prove that a functional $\mathbb{E}$ satisfies sublinearity if and only if it satisfies convexity and positive homogeneity.

Exercise 1.9 Suppose that all elements in $\mathcal{H}$ are bounded. Prove that the strongest sublinear expectation on $\mathcal{H}$ is

$$
\mathbb{E}^{\infty}[X]:=X^{*}=\sup _{\omega \in \Omega} X(\omega)
$$

Namely, all other sublinear expectations are dominated by $\mathbb{E}^{\infty}[\cdot]$.

\section{$\S 2 \quad$ Representation of a Sublinear Expectation}

A sublinear expectation can be expressed as a supremum of linear expectations.

Theorem 2.1 Let $\mathbb{E}$ be a functional defined on a linear space $\mathcal{H}$ satisfying subadditivity and positive homogeneity. Then there exists a family of linear functionals $\left\{E_{\theta}: \theta \in \Theta\right\}$ defined on $\mathcal{H}$ such that

$$
\mathbb{E}[X]=\sup _{\theta \in \Theta} E_{\theta}[X] \text { for } X \in \mathcal{H}
$$

and, for each $X \in \mathcal{H}$, there exists $\theta_{X} \in \Theta$ such that $\mathbb{E}[X]=E_{\theta_{X}}[X]$.

Furthermore, if $\mathbb{E}$ is a sublinear expectation, then the corresponding $E_{\theta}$ is a linear expectation.

Proof. Let $\mathcal{Q}=\left\{E_{\theta}: \theta \in \Theta\right\}$ be the family of all linear functionals dominated by $\mathbb{E}$, i.e., $E_{\theta}[X] \leq \mathbb{E}[X]$, for all $X \in \mathcal{H}, E_{\theta} \in \mathcal{Q}$.

We first prove that $\mathcal{Q}$ is non empty. For a given $X \in \mathcal{H}$, we set $L=\{a X: a \in$ $\mathbb{R}\}$ which is a subspace of $\mathcal{H}$. We define $I: L \rightarrow \mathbb{R}$ by $I[a X]=a \mathbb{E}[X], \forall a \in \mathbb{R}$, then $I[\cdot]$ forms a linear functional on $\mathcal{H}$ and $I \leq \mathbb{E}$ on $L$. Since $\mathbb{E}[\cdot]$ is subadditive and positively homogeneous, by Hahn-Banach theorem (see Appendix A), there exists a linear functional $E$ on $\mathcal{H}$ such that $E=I$ on $L$ and $E \leq \mathbb{E}$ on $\mathcal{H}$. Thus $E$ is a linear functional dominated by $\mathbb{E}$ such that $\mathbb{E}[X]=E[X]$.

We now define

$$
\mathbb{E}_{\Theta}[X]:=\sup _{\theta \in \Theta} E_{\theta}[X] \text { for } X \in \mathcal{H} .
$$

It is clear that $\mathbb{E}_{\Theta}=\mathbb{E}$.

Furthermore, if $\mathbb{E}$ is a sublinear expectation, then we have that, for each nonnegative element $X \in \mathcal{H}, E[X]=-E[-X] \geq-\mathbb{E}[-X] \geq 0$. For each $c \in \mathbb{R}$, $-E[c]=E[-c] \leq \mathbb{E}[-c]=-c$ and $E[c] \leq \mathbb{E}[c]=c$, so we get $E[c]=c$. Thus $E$ is a linear expectation. The proof is complete. 
Remark 2.2 It is important to observe that the above linear expectation $E_{\theta}$ is only "finitely additive". A sufficient condition for the $\sigma$-additivity of $E_{\theta}$ is to assume that $\mathbb{E}\left[X_{i}\right] \rightarrow 0$ for each sequence $\left\{X_{i}\right\}_{i=1}^{\infty}$ of $\mathcal{H}$ such that $X_{i}(\omega) \downarrow 0$ for each $\omega$. In this case, it is clear that $E_{\theta}\left[X_{i}\right] \rightarrow 0$. Thus we can apply the well-known Daniell-Stone Theorem (see Theorem 3.3 in Appendix B) to find a $\sigma$-additive probability measure $P_{\theta}$ on $(\Omega, \sigma(\mathcal{H}))$ such that

$$
E_{\theta}[X]=\int_{\Omega} X(\omega) d P_{\theta}, \quad X \in \mathcal{H} .
$$

The corresponding model uncertainty of probabilities is the subset $\left\{P_{\theta}: \theta \in \Theta\right\}$, and the corresponding uncertainty of distributions for an n-dimensional random vector $X$ in $\mathcal{H}$ is $\left\{F_{X}(\theta, A):=P_{\theta}(X \in A): A \in \mathcal{B}\left(\mathbb{R}^{n}\right)\right\}$.

In many situation, we may concern the probability uncertainty, and the probability maybe only finitely additive. So next we will give another version of the above representation theorem.

Let $\mathcal{P}_{f}$ be the collection of all finitely additive probability measures on $(\Omega, \mathcal{F})$, we consider $\mathbb{L}_{0}^{\infty}(\Omega, \mathcal{F})$ the collection of risk positions with finite values, which consists risk positions $X$ of the form

$$
X(\omega)=\sum_{i=1}^{N} x_{i} \mathbf{I}_{A_{i}}(\omega), x_{i} \in \mathbb{R}, A_{i} \in \mathcal{F}, i=1, \cdots, N .
$$

It is easy to check that, under the norm $\|\cdot\|_{\infty}, \mathbb{L}_{0}^{\infty}(\Omega, \mathcal{F})$ is dense in $\mathbb{L}^{\infty}(\Omega, \mathcal{F})$. For a fixed $Q \in \mathcal{P}_{f}$ and $X \in \mathbb{L}_{0}^{\infty}(\Omega, \mathcal{F})$ we define

$$
E_{Q}[X]=E_{Q}\left[\sum_{i=1}^{N} x_{i} \mathbf{I}_{A_{i}}(\omega)\right]:=\sum_{i=1}^{N} x_{i} Q\left(A_{i}\right)=\int_{\Omega} X(\omega) Q(d \omega) .
$$

$E_{Q}: \mathbb{L}_{0}^{\infty}(\Omega, \mathcal{F}) \rightarrow \mathbb{R}$ is a linear functional. It is easy to check that $E_{Q}$ satisfies (i) monotonicity and (ii) constant preserving. It is also continuous under $\|X\|_{\infty}$.

$$
\left|E_{Q}[X]\right| \leq \sup _{\omega \in \Omega}|X(\omega)|=\|X\|_{\infty} .
$$

Since $\mathbb{L}_{0}^{\infty}$ is dense in $\mathbb{L}^{\infty}$ we then can extend $E_{Q}$ from $\mathbb{L}_{0}^{\infty}$ to a linear continuous functional on $\mathbb{L}^{\infty}(\Omega, \mathcal{F})$.

Proposition 2.3 The linear functional $E_{Q}[\cdot]: \mathbb{L}^{\infty}(\Omega, \mathcal{F}) \rightarrow \mathbb{R}$ satisfies (i) and (ii). Inversely each linear functional $\eta(\cdot): \mathbb{L}^{\infty}(\Omega, \mathcal{F}) \rightarrow \mathbb{R}$ satisfying (i) and (ii) induces a finitely additive probability measure via $Q_{\eta}(A)=\eta\left(\mathbf{I}_{A}\right), A \in \mathcal{F}$. The corresponding expectation is $\eta$ itself

$$
\eta(X)=\int_{\Omega} X(\omega) Q_{\eta}(d \omega) .
$$


Theorem 2.4 A sublinear expectation $\mathbb{E}$ has the following representation: there exists a subset $\mathcal{Q} \subset \mathcal{P}_{f}$, such that

$$
\mathbb{E}[X]=\sup _{Q \in \mathcal{Q}} E_{Q}[X] \text { for } X \in \mathcal{H} .
$$

Proof. By Theorem 2.1, we have

$$
\mathbb{E}[X]=\sup _{\theta \in \Theta} E_{\theta}[X] \text { for } X \in \mathcal{H},
$$

where $E_{\theta}$ is a linear expectation on $\mathcal{H}$ for fixed $\theta \in \Theta$.

We can define a new sublinear expectation on $\mathbb{L}^{\infty}(\Omega, \sigma(\mathcal{H}))$ by

$$
\tilde{\mathbb{E}}_{\theta}[X]:=\inf \left\{E_{\theta}[Y] ; Y \geq X, Y \in \mathcal{H}\right\} .
$$

It is not difficult to check that $\tilde{\mathbb{E}}_{\theta}$ is a sublinear expectation on $\mathbb{L}^{\infty}(\Omega, \sigma(\mathcal{H}))$, where $\sigma(\mathcal{H})$ is the smallest $\sigma$-algebra generated by $\mathcal{H}$. We also have $E_{\theta} \leq \widetilde{\mathbb{E}}_{\theta}$ on $\mathcal{H}$, by Hahn-Banach theorem, $E_{\theta}$ can be extended from $\mathcal{H}$ to $\mathbb{L}^{\infty}(\Omega, \sigma(\mathcal{H}))$, by Proposition 2.3, there exists $Q \in \mathcal{P}_{f}$, such that

$$
E_{\theta}[X]=E_{Q}[X] \text { for } X \in \mathcal{H} .
$$

So there exists $\mathcal{Q} \subset \mathcal{P}_{f}$, such that

$$
\mathbb{E}[X]=\sup _{Q \in \mathcal{Q}} E_{Q}[X] \text { for } X \in \mathcal{H} .
$$

Exercise 2.5 Prove that $\tilde{\mathbb{E}}_{\theta}$ is a sublinear expectation.

\section{$\S 3 \quad$ Distributions, Independence and Product Spaces}

We now give the notion of distributions of random variables under sublinear expectations.

Let $X=\left(X_{1}, \cdots, X_{n}\right)$ be a given $n$-dimensional random vector on a nonlinear expectation space $(\Omega, \mathcal{H}, \mathbb{E})$. We define a functional on $C_{\text {l.Lip }}\left(\mathbb{R}^{n}\right)$ by

$$
\mathbb{F}_{X}[\varphi]:=\mathbb{E}[\varphi(X)]: \varphi \in C_{l . L i p}\left(\mathbb{R}^{n}\right) \rightarrow \mathbb{R} .
$$

The triple $\left(\mathbb{R}^{n}, C_{\text {l.Lip }}\left(\mathbb{R}^{n}\right), \mathbb{F}_{X}\right)$ forms a nonlinear expectation space. $\mathbb{F}_{X}$ is called the distribution of $X$ under $\mathbb{E}$. This notion is very useful for a sublinear expectation space $\mathbb{E}$. In this case $\mathbb{F}_{X}$ is also a sublinear expectation. Furthermore we can prove that (see Remark 2.2), there exists a family of probability measures $\left\{F_{X}^{\theta}(\cdot)\right\}_{\theta \in \Theta}$ defined on $\left(\mathbb{R}^{n}, \mathcal{B}\left(\mathbb{R}^{n}\right)\right)$ such that

$$
\mathbb{F}_{X}[\varphi]=\sup _{\theta \in \Theta} \int_{\mathbb{R}^{n}} \varphi(x) F_{X}^{\theta}(d x), \text { for each } \varphi \in C_{b . L i p}\left(\mathbb{R}^{n}\right) .
$$

Thus $\mathbb{F}_{X}[\cdot]$ characterizes the uncertainty of the distributions of $X$. 
Definition 3.1 Let $X_{1}$ and $X_{2}$ be two $n$-dimensional random vectors defined on nonlinear expectation spaces $\left(\Omega_{1}, \mathcal{H}_{1}, \mathbb{E}_{1}\right)$ and $\left(\Omega_{2}, \mathcal{H}_{2}, \mathbb{E}_{2}\right)$, respectively. They are called identically distributed, denoted by $X_{1} \stackrel{d}{=} X_{2}$, if

$$
\mathbb{E}_{1}\left[\varphi\left(X_{1}\right)\right]=\mathbb{E}_{2}\left[\varphi\left(X_{2}\right)\right] \quad \text { for } \varphi \in C_{l . L i p}\left(\mathbb{R}^{n}\right) .
$$

It is clear that $X_{1} \stackrel{d}{=} X_{2}$ if and only if their distributions coincide. We say that the distribution of $X_{1}$ is stronger than that of $X_{2}$ if $\mathbb{E}_{1}\left[\varphi\left(X_{1}\right)\right] \geq \mathbb{E}_{2}\left[\varphi\left(X_{2}\right)\right]$, for each $\varphi \in C_{l . L i p}\left(\mathbb{R}^{n}\right)$.

Remark 3.2 In the case of sublinear expectations, $X_{1} \stackrel{d}{=} X_{2}$ implies that the uncertainty subsets of distributions of $X_{1}$ and $X_{2}$ are the same, e.g., in the framework of Remark 2.2,

$$
\left\{F_{X_{1}}\left(\theta_{1}, \cdot\right): \theta_{1} \in \Theta_{1}\right\}=\left\{F_{X_{2}}\left(\theta_{2}, \cdot\right): \theta_{2} \in \Theta_{2}\right\} .
$$

Similarly if the distribution of $X_{1}$ is stronger than that of $X_{2}$, then

$$
\left\{F_{X_{1}}\left(\theta_{1}, \cdot\right): \theta_{1} \in \Theta_{1}\right\} \supset\left\{F_{X_{2}}\left(\theta_{2}, \cdot\right): \theta_{2} \in \Theta_{2}\right\} .
$$

The distribution of $X \in \mathcal{H}$ has the following four typical parameters:

$$
\bar{\mu}:=\mathbb{E}[X], \quad \underline{\mu}:=-\mathbb{E}[-X], \quad \bar{\sigma}^{2}:=\mathbb{E}\left[X^{2}\right], \quad \underline{\sigma}^{2}:=-\mathbb{E}\left[-X^{2}\right] .
$$

The intervals $[\underline{\mu}, \bar{\mu}]$ and $\left[\underline{\sigma}^{2}, \bar{\sigma}^{2}\right]$ characterize the mean-uncertainty and the variance-uncertainty of $X$ respectively.

A natural question is: can we find a family of distribution measures to represent the above sublinear distribution of $X$ ? The answer is affirmative:

Lemma 3.3 Let $(\Omega, \mathcal{H}, \mathbb{E})$ be a sublinear expectation space. Let $X \in \mathcal{H}^{d}$ be given. Then for each sequence $\left\{\varphi_{n}\right\}_{n=1}^{\infty} \subset C_{\text {l.Lip }}\left(\mathbb{R}^{d}\right)$ satisfying $\varphi_{n} \downarrow 0$, we have $\mathbb{E}\left[\varphi_{n}(X)\right] \downarrow 0$.

Proof. For each fixed $N>0$,

$$
\varphi_{n}(x) \leq k_{n}^{N}+\varphi_{1}(x) \mathbf{I}_{[|x|>N]} \leq k_{n}^{N}+\frac{\varphi_{1}(x)|x|}{N} \text { for each } x \in \mathbb{R}^{d \times m},
$$

where $k_{n}^{N}=\max _{|x| \leq N} \varphi_{n}(x)$. We then have

$$
\mathbb{E}\left[\varphi_{n}(X)\right] \leq k_{n}^{N}+\frac{1}{N^{\delta}} \mathbb{E}\left[\varphi_{1}(X)|X|^{\delta}\right] .
$$

It follows from $\varphi_{n} \downarrow 0$ that $k_{n}^{N} \downarrow 0$. Thus we have $\lim _{n \rightarrow \infty} \mathbb{E}\left[\varphi_{n}(X)\right] \leq$ $\frac{C}{N} \mathbb{E}\left[\varphi_{1}(X)|X|\right]$. Since $N$ can be arbitrarily large, we get $\mathbb{E}\left[\varphi_{n}(X)\right] \downarrow 0$.

Lemma 3.4 Let $(\Omega, \mathcal{H}, \mathbb{E})$ be a sublinear expectation space and let $\mathbb{F}_{X}[\varphi]:=$ $\mathbb{E}[\varphi(X)]$ be the sublinear distribution of $X \in \mathcal{H}^{d}$. Then there exists a family of probability measures $\left\{F_{\theta}\right\}_{\theta \in \Theta}$ defined on $\left(\mathbb{R}^{d}, \mathcal{B}\left(\mathbb{R}^{d}\right)\right)$ such that

$$
\mathbb{F}_{X}[\varphi]=\sup _{\theta \in \Theta} \int_{\mathbb{R}^{d}} \varphi(x) F_{\theta}(d x), \quad \varphi \in C_{l, L i p}\left(\mathbb{R}^{d}\right) .
$$


Proof. By the representation theorem, for the sublinear expectation $\mathbb{F}_{X}[\varphi]$ defined on $\left(\mathbb{R}^{d}, C_{\text {l.Lip }}\left(\mathbb{R}^{n}\right)\right)$, there exists a family of linear expectations $\left\{f_{\theta}\right\}_{\theta \in \Theta}$ on $\left(\mathbb{R}^{d}, C_{\text {l.Lip }}\left(\mathbb{R}^{n}\right)\right)$ such that

$$
\mathbb{F}_{X}[\varphi]=\sup _{\theta \in \Theta} f_{\theta}[\varphi], \varphi \in C_{l . L i p}\left(\mathbb{R}^{n}\right) .
$$

By the above lemma, for each sequence $\left\{\varphi_{n}\right\}_{n=1}^{\infty}$ in $C_{b . L i p}\left(\mathbb{R}^{n}\right)$ such that $\varphi_{n} \downarrow 0$ on $\mathbb{R}^{d}, \mathbb{F}_{X}\left[\varphi_{n}\right] \downarrow 0$, thus $f_{\theta}\left[\varphi_{n}\right] \downarrow 0$ for each $\theta \in \Theta$. It follows from Daniell-Stone Theorem (see Theorem 3.3 in Appendix B) that, for each $\theta \in \Theta$, there exists a unique probability measure $F_{\theta}(\cdot)$ on $\left(\mathbb{R}^{d}, \sigma\left(C_{b . L i p}\left(\mathbb{R}^{d}\right)\right)=\left(\mathbb{R}^{d}, \mathcal{B}\left(\mathbb{R}^{d}\right)\right)\right.$, such that $f_{\theta}[\varphi]=\int_{\mathbb{R}^{d}} \varphi(x) F_{\theta}(d x)$. Thus we have (3.2).

Remark 3.5 The above lemma tells us that in fact the sublinear distribution $\mathbb{F}_{X}$ of $X$ characterizes the uncertainty of distribution of $X$ which is an subset of distributions $\left\{F_{\theta}\right\}_{\theta \in \Theta}$.

The following property is very useful in our sublinear expectation theory.

Proposition 3.6 Let $(\Omega, \mathcal{H}, \mathbb{E})$ be a sublinear expectation space and $X, Y$ be two random variables such that $\mathbb{E}[Y]=-\mathbb{E}[-Y]$, i.e., $Y$ has no mean-uncertainty. Then we have

$$
\mathbb{E}[X+\alpha Y]=\mathbb{E}[X]+\alpha \mathbb{E}[Y] \text { for } \alpha \in \mathbb{R} .
$$

In particular, if $\mathbb{E}[Y]=\mathbb{E}[-Y]=0$, then $\mathbb{E}[X+\alpha Y]=\mathbb{E}[X]$.

Proof. We have

$$
\mathbb{E}[\alpha Y]=\alpha^{+} \mathbb{E}[Y]+\alpha^{-} \mathbb{E}[-Y]=\alpha^{+} \mathbb{E}[Y]-\alpha^{-} \mathbb{E}[Y]=\alpha \mathbb{E}[Y] \text { for } \alpha \in \mathbb{R} .
$$

Thus

$\mathbb{E}[X+\alpha Y] \leq \mathbb{E}[X]+\mathbb{E}[\alpha Y]=\mathbb{E}[X]+\alpha \mathbb{E}[Y]=\mathbb{E}[X]-\mathbb{E}[-\alpha Y] \leq \mathbb{E}[X+\alpha Y]$

A more general form of the above proposition is:

Proposition 3.7 We make the same assumptions as the previous proposition. Let $\tilde{\mathbb{E}}$ be a nonlinear expectation on $(\Omega, \mathcal{H})$ dominated by the sublinear expectation $\mathbb{E}$ in the sense of (1.1). If $\mathbb{E}[Y]=\mathbb{E}[-Y]$, then we have

$$
\tilde{\mathbb{E}}[\alpha Y]=\alpha \tilde{\mathbb{E}}[Y]=\alpha \mathbb{E}[Y], \quad \alpha \in \mathbb{R}
$$

as well as

$$
\tilde{\mathbb{E}}[X+\alpha Y]=\tilde{\mathbb{E}}[X]+\alpha \tilde{\mathbb{E}}[Y], \quad X \in \mathcal{H}, \quad \alpha \in \mathbb{R} .
$$

In particular

$$
\tilde{\mathbb{E}}[X+c]=\tilde{\mathbb{E}}[X]+c, \text { for } c \in \mathbb{R} .
$$


Proof. We have

$$
-\tilde{\mathbb{E}}[Y]=\tilde{\mathbb{E}}[0]-\tilde{\mathbb{E}}[Y] \leq \mathbb{E}[-Y]=-\mathbb{E}[Y] \leq-\tilde{\mathbb{E}}[Y]
$$

and

$$
\begin{aligned}
\mathbb{E}[Y] & =-\mathbb{E}[-Y] \leq-\tilde{\mathbb{E}}[-Y] \\
& =\tilde{\mathbb{E}}[0]-\tilde{\mathbb{E}}[-Y] \leq \mathbb{E}[Y] .
\end{aligned}
$$

From the above relations we have $\tilde{\mathbb{E}}[Y]=\mathbb{E}[Y]=-\tilde{\mathbb{E}}[-Y]$ and thus (3.3). Still by the domination,

$$
\begin{aligned}
& \tilde{\mathbb{E}}[X+\alpha Y]-\tilde{\mathbb{E}}[X] \leq \mathbb{E}[\alpha Y] \\
& \tilde{\mathbb{E}}[X]-\tilde{\mathbb{E}}[X+\alpha Y] \leq \mathbb{E}[-\alpha Y]=-\mathbb{E}[\alpha Y] .
\end{aligned}
$$

Thus (3.4) holds.

Definition 3.8 A sequence of n-dimensional random vectors $\left\{\eta_{i}\right\}_{i=1}^{\infty}$ defined on a sublinear expectation space $(\Omega, \mathcal{H}, \mathbb{E})$ is said to converge in distribution (or converge in law) under $\mathbb{E}$ if for each $\varphi \in C_{b . L i p}\left(\mathbb{R}^{n}\right)$, the sequence $\left\{\mathbb{E}\left[\varphi\left(\eta_{i}\right)\right]\right\}_{i=1}^{\infty}$ converges.

The following result is easy to check.

Proposition 3.9 Let $\left\{\eta_{i}\right\}_{i=1}^{\infty}$ converge in law in the above sense. Then the mapping $\mathbb{F}[\cdot]: C_{\text {b.Lip }}\left(\mathbb{R}^{n}\right) \rightarrow \mathbb{R}$ defined by

$$
\mathbb{F}[\varphi]:=\lim _{i \rightarrow \infty} \mathbb{E}\left[\varphi\left(\eta_{i}\right)\right] \text { for } \varphi \in C_{b . \operatorname{Lip}}\left(\mathbb{R}^{n}\right)
$$

is a sublinear expectation defined on $\left(\mathbb{R}^{n}, C_{b . L i p}\left(\mathbb{R}^{n}\right)\right)$.

The following notion of independence plays a key role in the nonlinear expectation theory.

Definition 3.10 In a nonlinear expectation space $(\Omega, \mathcal{H}, \mathbb{E})$, a random vector $Y \in \mathcal{H}^{n}$ is said to be independent from another random vector $X \in \mathcal{H}^{m}$ under $\mathbb{E}[\cdot]$ if for each test function $\varphi \in C_{l . L i p}\left(\mathbb{R}^{m+n}\right)$ we have

$$
\mathbb{E}[\varphi(X, Y)]=\mathbb{E}\left[\mathbb{E}[\varphi(x, Y)]_{x=X}\right] .
$$

Remark 3.11 In particular, for a sublinear expectation space $(\Omega, \mathcal{H}, \mathbb{E}), Y$ is independent from $X$ means that the uncertainty of distributions $\left\{F_{Y}(\theta, \cdot): \theta \in\right.$ $\Theta\}$ of $Y$ does not change after the realization of $X=x$. In other words, the "conditional sublinear expectation" of $Y$ with respect to $X$ is $\mathbb{E}[\varphi(x, Y)]_{x=X}$. In the case of linear expectation, this notion of independence is just the classical one.

Remark 3.12 It is important to note that under sublinear expectations the condition " $Y$ is independent from $X$ " does not imply automatically that " $X$ is independent from $Y$ ". 
Example 3.13 We consider a case where $\mathbb{E}$ is a sublinear expectation and $X, Y \in \mathcal{H}$ are identically distributed with $\mathbb{E}[X]=\mathbb{E}[-X]=0$ and $\bar{\sigma}^{2}=$ $\mathbb{E}\left[X^{2}\right]>\underline{\sigma}^{2}=-\mathbb{E}\left[-X^{2}\right]$. We also assume that $\mathbb{E}[|X|]=\mathbb{E}\left[X^{+}+X^{-}\right]>0$, thus $\mathbb{E}\left[X^{+}\right]=\frac{1}{2} \mathbb{E}[|X|+X]=\frac{1}{2} \mathbb{E}[|X|]>0$. In the case where $Y$ is independent from $X$, we have

$$
\mathbb{E}\left[X Y^{2}\right]=\mathbb{E}\left[X^{+} \bar{\sigma}^{2}-X^{-} \underline{\sigma}^{2}\right]=\left(\bar{\sigma}^{2}-\underline{\sigma}^{2}\right) \mathbb{E}\left[X^{+}\right]>0 .
$$

But if $X$ is independent from $Y$, we have

$$
\mathbb{E}\left[X Y^{2}\right]=0 .
$$

The independence property of two random vectors $X, Y$ involves only the "joint distribution" of $(X, Y)$. The following result tells us how to construct random vectors with given "marginal distributions" and with a specific direction of independence.

Definition 3.14 Let $\left(\Omega_{i}, \mathcal{H}_{i}, \mathbb{E}_{i}\right), i=1,2$ be two sublinear (resp. nonlinear) expectation spaces. We denote

$$
\begin{gathered}
\mathcal{H}_{1} \otimes \mathcal{H}_{2}:=\left\{Z\left(\omega_{1}, \omega_{2}\right)=\varphi\left(X\left(\omega_{1}\right), Y\left(\omega_{2}\right)\right):\left(\omega_{1}, \omega_{2}\right) \in \Omega_{1} \times \Omega_{2},\right. \\
\left.(X, Y) \in \mathcal{H}_{1}^{m} \times \mathcal{H}_{2}^{n}, \varphi \in C_{l . L i p}\left(\mathbb{R}^{m+n}\right)\right\},
\end{gathered}
$$

and, for each random variable of the above form $Z\left(\omega_{1}, \omega_{2}\right)=\varphi\left(X\left(\omega_{1}\right), Y\left(\omega_{2}\right)\right)$,

$$
\left(\mathbb{E}_{1} \otimes \mathbb{E}_{2}\right)[Z]:=\mathbb{E}_{1}[\bar{\varphi}(X)] \text {, where } \bar{\varphi}(x):=\mathbb{E}_{2}[\varphi(x, Y)], x \in \mathbb{R}^{m} .
$$

It is easy to check that the triple $\left(\Omega_{1} \times \Omega_{2}, \mathcal{H}_{1} \otimes \mathcal{H}_{2}, \mathbb{E}_{1} \otimes \mathbb{E}_{2}\right)$ forms a sublinear (resp. nonlinear) expectation space. We call it the product space of sublinear (resp. nonlinear) expectation spaces $\left(\Omega_{1}, \mathcal{H}_{1}, \mathbb{E}_{1}\right)$ and $\left(\Omega_{2}, \mathcal{H}_{2}, \mathbb{E}_{2}\right)$. In this way, we can define the product space

$$
\left(\prod_{i=1}^{n} \Omega_{i}, \bigotimes_{i=1}^{n} \mathcal{H}_{i}, \bigotimes_{i=1}^{n} \mathbb{E}_{i}\right)
$$

of given sublinear (resp. nonlinear) expectation spaces $\left(\Omega_{i}, \mathcal{H}_{i}, \mathbb{E}_{i}\right), i=$ $1,2, \cdots, n$. In particular, when $\left(\Omega_{i}, \mathcal{H}_{i}, \mathbb{E}_{i}\right)=\left(\Omega_{1}, \mathcal{H}_{1}, \mathbb{E}_{1}\right)$ we have the product space of the form $\left(\Omega_{1}^{n}, \mathcal{H}_{1}^{\otimes n}, \mathbb{E}_{1}^{\otimes n}\right)$.

Let $X, \bar{X}$ be two $n$-dimensional random vectors on a sublinear (resp. nonlinear) expectation space $(\Omega, \mathcal{H}, \mathbb{E}) . \quad \bar{X}$ is called an independent copy of $X$ if $\bar{X} \stackrel{d}{=} X$ and $\bar{X}$ is independent from $X$.

The following property is easy to check.

Proposition 3.15 Let $X_{i}$ be an $n_{i}$-dimensional random vector on sublinear (resp. nonlinear) expectation space $\left(\Omega_{i}, \mathcal{H}_{i}, \mathbb{E}_{i}\right)$ for $i=1, \cdots, n$, respectively. We denote

$$
Y_{i}\left(\omega_{1}, \cdots, \omega_{n}\right):=X_{i}\left(\omega_{i}\right), \quad i=1, \cdots, n .
$$


Then $Y_{i}, i=1, \cdots, n$, are random vectors on $\left(\prod_{i=1}^{n} \Omega_{i}, \bigotimes_{i=1}^{n} \mathcal{H}_{i}, \bigotimes_{i=1}^{n} \mathbb{E}_{i}\right)$. Moreover we have $Y_{i} \stackrel{d}{=} X_{i}$ and $Y_{i+1}$ is independent from $\left(Y_{1}, \cdots, Y_{i}\right)$, for each $i$.

Furthermore, if $\left(\Omega_{i}, \mathcal{H}_{i}, \mathbb{E}_{i}\right)=\left(\Omega_{1}, \mathcal{H}_{1}, \mathbb{E}_{1}\right)$ and $X_{i} \stackrel{d}{=} X_{1}$, for all $i$, then we also have $Y_{i} \stackrel{d}{=} Y_{1}$. In this case $Y_{i}$ is said to be an independent copy of $Y_{1}$ for $i=2, \cdots, n$.

Remark 3.16 In the above construction the integer $n$ can be also infinite. In this case each random variable $X \in \bigotimes_{i=1}^{\infty} \mathcal{H}_{i}$ belongs to $\left(\prod_{i=1}^{k} \Omega_{i}, \bigotimes_{i=1}^{k} \mathcal{H}_{i}, \bigotimes_{i=1}^{k} \mathbb{E}_{i}\right)$ for some positive integer $k<\infty$ and

$$
\bigotimes_{i=1}^{\infty} \mathbb{E}_{i}[X]:=\bigotimes_{i=1}^{k} \mathbb{E}_{i}[X]
$$

Remark 3.17 The situation " $Y$ is independent from $X$ " often appears when $Y$ occurs after $X$, thus a robust expectation should take the information of $X$ into account.

Exercise 3.18 Suppose $X, Y \in \mathcal{H}^{d}$ and $Y$ is an independent copy of $X$. Prove that for each $a \in \mathbb{R}, b \in \mathbb{R}^{d}, a+\langle b, Y\rangle$ is an independent copy of $a+\langle b, X\rangle$.

In a sublinear expectation space we have:

Example 3.19 We consider a situation where two random variables $X$ and $Y$ in $\mathcal{H}$ are identically distributed and their common distribution is

$$
\mathbb{F}_{X}[\varphi]=\mathbb{F}_{Y}[\varphi]=\sup _{\theta \in \Theta} \int_{\mathbb{R}} \varphi(y) F(\theta, d y) \text { for } \varphi \in C_{l . L i p}(\mathbb{R}),
$$

where for each $\theta \in \Theta,\{F(\theta, A)\}_{A \in \mathcal{B}(\mathbb{R})}$ is a probability measure on $(\mathbb{R}, \mathcal{B}(\mathbb{R}))$. In this case, " $Y$ is independent from $X$ " means that the joint distribution of $X$ and $Y$ is

$$
\mathbb{F}_{X, Y}[\psi]=\sup _{\theta_{1} \in \Theta} \int_{\mathbb{R}}\left[\sup _{\theta_{2} \in \Theta} \int_{\mathbb{R}} \psi(x, y) F\left(\theta_{2}, d y\right)\right] F\left(\theta_{1}, d x\right) \text { for } \psi \in C_{\text {l.Lip }}\left(\mathbb{R}^{2}\right) .
$$

Exercise 3.20 Let $(\Omega, \mathcal{H}, \mathbb{E})$ be a sublinear expectation space. Prove that if $\mathbb{E}[\varphi(X)]=\mathbb{E}[\varphi(Y)]$ for any $\varphi \in C_{b, \text { Lip }}$, then it still holds for any $\varphi \in C_{l, \text { Lip }}$. That is, we can replace $\varphi \in C_{l, L i p}$ in Definition 3.1 by $\varphi \in C_{b, L i p}$.

\section{$\S 4$ Completion of Sublinear Expectation Spaces}

Let $(\Omega, \mathcal{H}, \mathbb{E})$ be a sublinear expectation space. We have the following useful inequalities.

We first give the following well-known inequalities. 
Lemma 4.1 For $r>0$ and $1<p, q<\infty$ with $\frac{1}{p}+\frac{1}{q}=1$, we have

$$
\begin{aligned}
|a+b|^{r} & \leq \max \left\{1,2^{r-1}\right\}\left(|a|^{r}+|b|^{r}\right) \quad \text { for } a, b \in \mathbb{R}, \\
|a b| & \leq \frac{|a|^{p}}{p}+\frac{|b|^{q}}{q} .
\end{aligned}
$$

Proposition 4.2 For each $X, Y \in \mathcal{H}$, we have

$$
\begin{aligned}
\mathbb{E}\left[|X+Y|^{r}\right] & \leq 2^{r-1}\left(\mathbb{E}\left[|X|^{r}\right]+\mathbb{E}\left[|Y|^{r}\right]\right), \\
\mathbb{E}[|X Y|] & \leq\left(\mathbb{E}\left[|X|^{p}\right]\right)^{1 / p} \cdot\left(\mathbb{E}\left[|Y|^{q}\right]\right)^{1 / q}, \\
\left(\mathbb{E}\left[|X+Y|^{p}\right]\right)^{1 / p} & \leq\left(\mathbb{E}\left[|X|^{p}\right]\right)^{1 / p}+\left(\mathbb{E}\left[|Y|^{p}\right]\right)^{1 / p},
\end{aligned}
$$

where $r \geq 1$ and $1<p, q<\infty$ with $\frac{1}{p}+\frac{1}{q}=1$.

In particular, for $1 \leq p<p^{\prime}$, we have $\left(\mathbb{E}\left[|X|^{p}\right]\right)^{1 / p} \leq\left(\mathbb{E}\left[|X|^{p^{\prime}}\right]\right)^{1 / p^{\prime}}$.

Proof. The inequality (4.8) follows from (4.6).

For the case $\mathbb{E}\left[|X|^{p}\right] \cdot \mathbb{E}\left[|Y|^{q}\right]>0$, we set

$$
\xi=\frac{X}{\left(\mathbb{E}\left[\left.|X|\right|^{p}\right]\right)^{1 / p}}, \quad \eta=\frac{Y}{\left(\mathbb{E}\left[|Y|^{q}\right]\right)^{1 / q}} .
$$

By (4.7) we have

$$
\begin{aligned}
\mathbb{E}[|\xi \eta|] & \leq \mathbb{E}\left[\frac{|\xi|^{p}}{p}+\frac{|\eta|^{q}}{q}\right] \leq \mathbb{E}\left[\frac{|\xi|^{p}}{p}\right]+\mathbb{E}\left[\frac{|\eta|^{q}}{q}\right] \\
& =\frac{1}{p}+\frac{1}{q}=1 .
\end{aligned}
$$

Thus (4.9) follows.

For the case $\mathbb{E}\left[|X|^{p}\right] \cdot \mathbb{E}\left[|Y|^{q}\right]=0$, we consider $\mathbb{E}\left[|X|^{p}\right]+\varepsilon$ and $\mathbb{E}\left[|Y|^{q}\right]+\varepsilon$ for $\varepsilon>0$. Applying the above method and letting $\varepsilon \rightarrow 0$, we get (4.9).

We now prove (4.10). We only consider the case $\mathbb{E}\left[|X+Y|^{p}\right]>0$.

$$
\begin{aligned}
\mathbb{E}\left[|X+Y|^{p}\right]= & \mathbb{E}\left[|X+Y| \cdot|X+Y|^{p-1}\right] \\
\leq & \mathbb{E}\left[|X| \cdot|X+Y|^{p-1}\right]+\mathbb{E}\left[|Y| \cdot|X+Y|^{p-1}\right] \\
\leq & \left(\mathbb{E}\left[|X|^{p}\right]\right)^{1 / p} \cdot\left(\mathbb{E}\left[|X+Y|^{(p-1) q}\right]\right)^{1 / q} \\
& +\left(\mathbb{E}\left[|Y|^{p}\right]\right)^{1 / p} \cdot\left(\mathbb{E}\left[|X+Y|^{(p-1) q}\right]\right)^{1 / q} .
\end{aligned}
$$

Since $(p-1) q=p$, we have (4.10).

By (4.9), it is easy to deduce that $\left(\mathbb{E}\left[|X|^{p}\right]\right)^{1 / p} \leq\left(\mathbb{E}\left[|X|^{p^{\prime}}\right]\right)^{1 / p^{\prime}}$ for $1 \leq p<p^{\prime}$.

For each fixed $p \geq 1$, we observe that $\mathcal{H}_{0}^{p}=\left\{X \in \mathcal{H}, \mathbb{E}\left[|X|^{p}\right]=0\right\}$ is a linear subspace of $\mathcal{H}$. Taking $\mathcal{H}_{0}^{p}$ as our null space, we introduce the quotient 
space $\mathcal{H} / \mathcal{H}_{0}^{p}$. Observing that, for every $\{X\} \in \mathcal{H} / \mathcal{H}_{0}^{p}$ with a representation $X \in \mathcal{H}$, we can define an expectation $\mathbb{E}[\{X\}]:=\mathbb{E}[X]$ which is still a sublinear expectation. We set $\|X\|_{p}:=\left(\mathbb{E}\left[|X|^{p}\right]\right)^{\frac{1}{p}}$. By Proposition 4.2 it is easy to check that $\|\cdot\|_{p}$ forms a Banach norm on $\mathcal{H} / \mathcal{H}_{0}^{p}$. We extend $\mathcal{H} / \mathcal{H}_{0}^{p}$ to its completion $\hat{\mathcal{H}}_{p}$ under this norm, then $\left(\hat{\mathcal{H}}_{p},\|\cdot\|_{p}\right)$ is a Banach space. In particular, when $p=1$, we denote it by $(\hat{\mathcal{H}},\|\cdot\|)$.

For each $X \in \mathcal{H}$, the mappings

$$
X^{+}(\omega): \mathcal{H} \rightarrow \mathcal{H} \quad \text { and } \quad X^{-}(\omega): \mathcal{H} \rightarrow \mathcal{H}
$$

satisfy

$$
\left|X^{+}-Y^{+}\right| \leq|X-Y| \quad \text { and } \quad\left|X^{-}-Y^{-}\right|=\left|(-X)^{+}-(-Y)^{+}\right| \leq|X-Y| .
$$

Thus they are both contraction mappings under $\|\cdot\|_{p}$ and can be continuously extended to the Banach space $\left(\hat{\mathcal{H}}_{p},\|\cdot\|_{p}\right)$.

We can define the partial order " $\geq$ " in this Banach space.

Definition 4.3 An element $X$ in $(\hat{\mathcal{H}},\|\cdot\|)$ is said to be nonnegative, or $X \geq 0$, $0 \leq X$, if $X=X^{+}$. We also denote by $X \geq Y$, or $Y \leq X$, if $X-Y \geq 0$.

It is easy to check that $X \geq Y$ and $Y \geq X$ imply $X=Y$ on $\left(\hat{\mathcal{H}}_{p},\|\cdot\|_{p}\right)$. For each $X, Y \in \mathcal{H}$, note that

$$
|\mathbb{E}[X]-\mathbb{E}[Y]| \leq \mathbb{E}[|X-Y|] \leq\|X-Y\|_{p} .
$$

We then can define

Definition 4.4 The sublinear expectation $\mathbb{E}[\cdot]$ can be continuously extended to $\left(\hat{\mathcal{H}}_{p},\|\cdot\|_{p}\right)$ on which it is still a sublinear expectation. We still denote by $\left(\Omega, \hat{\mathcal{H}}_{p}, \mathbb{E}\right)$.

Let $\left(\Omega, \mathcal{H}, \mathbb{E}_{1}\right)$ be a nonlinear expectation space. $\mathbb{E}_{1}$ is said to be dominated by $\mathbb{E}$ if

$$
\mathbb{E}_{1}[X]-\mathbb{E}_{1}[Y] \leq \mathbb{E}[X-Y] \quad \text { for } X, Y \in \mathcal{H} .
$$

From this we can easily deduce that $\left|\mathbb{E}_{1}[X]-\mathbb{E}_{1}[Y]\right| \leq \mathbb{E}[|X-Y|]$, thus the nonlinear expectation $\mathbb{E}_{1}[\cdot]$ can be continuously extended to $\left(\hat{\mathcal{H}}_{p},\|\cdot\|_{p}\right)$ on which it is still a nonlinear expectation. We still denote by $\left(\Omega, \hat{\mathcal{H}}_{p}, \mathbb{E}_{1}\right)$.

Remark 4.5 It is important to note that $X_{1}, \cdots, X_{n} \in \hat{\mathcal{H}}$ does not imply $\varphi\left(X_{1}, \cdots, X_{n}\right) \in \hat{\mathcal{H}}$ for each $\varphi \in C_{l . L i p}\left(\mathbb{R}^{n}\right)$. Thus, when we talk about the notions of distributions, independence and product spaces on $(\Omega, \hat{\mathcal{H}}, \mathbb{E})$, the space $C_{\text {l.Lip }}\left(\mathbb{R}^{n}\right)$ is replaced by $C_{\text {b.Lip }}\left(\mathbb{R}^{n}\right)$ unless otherwise stated.

Exercise 4.6 Prove that the inequalities (4.8), (4.9), (4.10) still hold for $(\Omega, \hat{\mathcal{H}}, \mathbb{E})$. 


\section{$\S 5$ Coherent Measures of Risk}

Let the pair $(\Omega, \mathcal{H})$ be such that $\Omega$ is a set of scenarios and $\mathcal{H}$ is the collection of all possible risk positions in a financial market.

If $X \in \mathcal{H}$, then for each constant $c, X \vee c, X \wedge c$ are all in $\mathcal{H}$. One typical example in finance is that $X$ is the tomorrow's price of a stock. In this case, any European call or put options with strike price $K$ of forms $(S-K)^{+},(K-S)^{+}$ are in $\mathcal{H}$.

A risk supervisor is responsible for taking a rule to tell traders, securities companies, banks or other institutions under his supervision, which kind of risk positions is unacceptable and thus a minimum amount of risk capitals should be deposited to make the positions acceptable. The collection of acceptable positions is defined by

$$
\mathcal{A}=\{X \in \mathcal{H}: X \text { is acceptable }\} .
$$

This set has meaningful properties in economy.

Definition 5.1 A set $\mathcal{A}$ is called a coherent acceptable set if it satisfies

(i) Monotonicity:

$$
X \in \mathcal{A}, Y \geq X \text { imply } Y \in \mathcal{A} .
$$

(ii) $0 \in \mathcal{A}$ but $-1 \notin \mathcal{A}$.

(iii) Positive homogeneity

$$
X \in \mathcal{A} \text { implies } \lambda X \in \mathcal{A} \text { for } \lambda \geq 0 .
$$

(iv) Convexity:

$$
X, Y \in \mathcal{A} \text { imply } \alpha X+(1-\alpha) Y \in \mathcal{A} \text { for } \alpha \in[0,1] .
$$

Remark 5.2 (iii)+(iv) imply

(v) Sublinearity:

$$
X, Y \in \mathcal{A} \Rightarrow \mu X+\nu Y \in \mathcal{A} \text { for } \mu, \nu \geq 0 .
$$

Remark 5.3 If the set $\mathcal{A}$ only satisfies (i),(ii) and (iv), then $\mathcal{A}$ is called a convex acceptable set.

In this section we mainly study the coherent case. Once the rule of the acceptable set is fixed, the minimum requirement of risk deposit is then automatically determined.

Definition 5.4 Given a coherent acceptable set $\mathcal{A}$, the functional $\rho(\cdot)$ defined by

$$
\rho(X)=\rho_{\mathcal{A}}(X):=\inf \{m \in \mathbb{R}: m+X \in \mathcal{A}\}, \quad X \in \mathcal{H}
$$

is called the coherent risk measure related to $\mathcal{A}$. 
It is easy to see that

$$
\rho(X+\rho(X))=0 .
$$

Proposition 5.5 $\rho(\cdot)$ is a coherent risk measure satisfying four properties:

(i) Monotonicity: If $X \geq Y$ then $\rho(X) \leq \rho(Y)$.

(ii) Constant preserving: $\rho(1)=-\rho(-1)=-1$.

(iii) Sub-additivity: For each $X, Y \in \mathcal{H}, \quad \rho(X+Y) \leq \rho(X)+\rho(Y)$.

(iv) Positive homogeneity: $\rho(\lambda X)=\lambda \rho(X)$ for $\lambda \geq 0$.

Proof. (i), (ii) are obvious.

We now prove (iii). Indeed,

$$
\begin{aligned}
\rho(X+Y) & =\inf \{m \in \mathbb{R}: m+(X+Y) \in \mathcal{A}\} \\
& =\inf \{m+n: m, n \in \mathbb{R},(m+X)+(n+Y) \in \mathcal{A}\} \\
& \leq \inf \{m \in \mathbb{R}: m+X \in \mathcal{A}\}+\inf \{n \in \mathbb{R}: n+Y \in \mathcal{A}\} \\
& =\rho(X)+\rho(Y) .
\end{aligned}
$$

To prove (iv), in fact the case $\lambda=0$ is trivial; when $\lambda>0$,

$$
\begin{aligned}
\rho(\lambda X) & =\inf \{m \in \mathbb{R}: m+\lambda X \in \mathcal{A}\} \\
& =\lambda \inf \{n \in \mathbb{R}: n+X \in \mathcal{A}\}=\lambda \rho(X),
\end{aligned}
$$

where $n=m / \lambda$.

Obviously, if $\mathbb{E}$ is a sublinear expectation, we define $\rho(X):=\mathbb{E}[-X]$, then $\rho$ is a coherent risk measure. Inversely, if $\rho$ is a coherent risk measure, we define $\mathbb{E}[X]:=\rho(-X)$, then $\mathbb{E}$ is a sublinear expectation.

Exercise 5.6 Let $\rho(\cdot)$ be a coherent risk measure. Then we can inversely define

$$
\mathcal{A}_{\rho}:=\{X \in \mathcal{H}: \rho(X) \leq 0\} .
$$

Prove that $\mathcal{A}_{\rho}$ is a coherent acceptable set.

\section{Notes and Comments}

The sublinear expectation is also called the upper expectation (see Huber (1981) [61] in robust statistics), or the upper prevision in the theory of imprecise probabilities (see Walley (1991) [120] and a rich literature provided in the Notes of this book). To our knowledge, the Representation Theorem 2.1 was firstly obtained for the case where $\Omega$ is a finite set by [61], and this theorem was rediscovered independently by Artzner, Delbaen, Eber and Heath (1999) [3] and then by Delbaen (2002) 35] for the general $\Omega$. A typical example of dynamic nonlinear expectation, called $g$-expectation (small $g$ ), was introduced in Peng (1997) [92] in the framework of backward stochastic differential equations. Readers are 
referred to Briand, Coquet, Hu, Mémin and Peng (2000) [14], Chen (1998) 18], Chen and Epstein (2002) [19], Chen, Kulperger and Jiang (2003) 20], Chen and Peng (1998) [21] and (2000) [22], Coquet, Hu, Mémin and Peng (2001) [26] (2002) [27], Jiang (2004) 69], Jiang and Chen (2004) 70, 71], Peng (1999) [94] and (2004) [97], Peng and Xu (2003) [107] and Rosazza (2006) [112] for the further development of this theory. It seems that the notions of distributions and independence under nonlinear expectations were new. We think that these notions are perfectly adapted for the further development of dynamic nonlinear expectations. For other types of the related notions of distributions and independence under nonlinear expectations or non-additive probabilities, we refer to the Notes of the book [120] and the references listed in Marinacci (1999) 83] and Maccheroni and Marinacci (2005) 844]. Coherent risk measures can be also regarded as sublinear expectations defined on the space of risk positions in financial market. This notion was firstly introduced in [3]. Readers can be referred also to the well-known book of Föllmer and Schied (2004) [51] for the systematical presentation of coherent risk measures and convex risk measures. For the dynamic risk measure in continuous time, see [97] or [112], Barrieu and El Karoui (2004) 9] using $g$-expectations. Super-hedging and super pricing (see El Karoui and Quenez (1995) [43] and El Karoui, Peng and Quenez (1997) [44]) are also closely related to this formulation. 


\section{Chapter II}

\section{Law of Large Numbers and Central Limit Theorem}

In this chapter, we first introduce two types of fundamentally important distributions, namely, maximal distribution and $G$-normal distribution, in the theory of sublinear expectations. The former corresponds to constants and the latter corresponds to normal distribution in classical probability theory. We then present the law of large numbers (LLN) and central limit theorem (CLT) under sublinear expectations. It is worth pointing out that the limit in LLN is a maximal distribution and the limit in CLT is a $G$-normal distribution.

\section{$\S 1$ Maximal Distribution and $G$-normal Distri- bution}

We will firstly define a special type of very simple distributions which are frequently used in practice, known as "worst case risk measure".

Definition 1.1 (maximal distribution) $A$ d-dimensional random vector $\eta=$ $\left(\eta_{1}, \cdots, \eta_{d}\right)$ on a sublinear expectation space $(\Omega, \mathcal{H}, \mathbb{E})$ is called maximal distributed if there exists a bounded, closed and convex subset $\Gamma \subset \mathbb{R}^{d}$ such that

$$
\mathbb{E}[\varphi(\eta)]=\max _{y \in \Gamma} \varphi(y) .
$$

Remark 1.2 Here $\Gamma$ gives the degree of uncertainty of $\eta$. It is easy to check that this maximal distributed random vector $\eta$ satisfies

$$
a \eta+b \bar{\eta} \stackrel{d}{=}(a+b) \eta \quad \text { for } a, b \geq 0,
$$

where $\bar{\eta}$ is an independent copy of $\eta$. We will see later that in fact this relation characterizes a maximal distribution. Maximal distribution is also called "worst case risk measure" in finance. 
Remark 1.3 When $d=1$ we have $\Gamma=[\underline{\mu}, \bar{\mu}]$, where $\bar{\mu}=\mathbb{E}[\eta]$ and $\underline{\mu}=-\mathbb{E}[-\eta]$. The distribution of $\eta$ is

$$
\mathbb{F}_{\eta}[\varphi]=\mathbb{E}[\varphi(\eta)]=\sup _{\underline{\mu} \leq y \leq \bar{\mu}} \varphi(y) \quad \text { for } \varphi \in C_{l . L i p}(\mathbb{R}) .
$$

Recall a well-known characterization: $X \stackrel{d}{=} N(0, \Sigma)$ if and only if

$$
a X+b \bar{X} \stackrel{d}{=} \sqrt{a^{2}+b^{2}} X \text { for } a, b \geq 0,
$$

where $\bar{X}$ is an independent copy of $X$. The covariance matrix $\Sigma$ is defined by $\Sigma=E\left[X X^{T}\right]$. We now consider the so called $G$-normal distribution in probability model uncertainty situation. The existence, uniqueness and characterization will be given later.

Definition 1.4 (G-normal distribution) $A$ d-dimensional random vector $X=$ $\left(X_{1}, \cdots, X_{d}\right)^{T}$ on a sublinear expectation space $(\Omega, \mathcal{H}, \mathbb{E})$ is called (centralized) G-normal distributed if

$$
a X+b \bar{X} \stackrel{d}{=} \sqrt{a^{2}+b^{2}} X \quad \text { for } a, b \geq 0,
$$

where $\bar{X}$ is an independent copy of $X$.

Remark 1.5 Noting that $\mathbb{E}[X+\bar{X}]=2 \mathbb{E}[X]$ and $\mathbb{E}[X+\bar{X}]=\mathbb{E}[\sqrt{2} X]=$ $\sqrt{2} \mathbb{E}[X]$, we then have $\mathbb{E}[X]=0$. Similarly, we can prove that $\mathbb{E}[-X]=0$. Namely, $X$ has no mean-uncertainty.

The following property is easy to prove by the definition.

Proposition 1.6 Let $X$ be $G$-normal distributed. Then for each $A \in \mathbb{R}^{m \times d}$, $A X$ is also $G$-normal distributed. In particular, for each $\mathbf{a} \in \mathbb{R}^{d},\langle\mathbf{a}, X\rangle$ is a 1-dimensional $G$-normal distributed random variable, but its inverse is not true (see Exercise 1.15).

We denote by $\mathbb{S}(d)$ the collection of all $d \times d$ symmetric matrices. Let $X$ be $G$-normal distributed and $\eta$ be maximal distributed $d$-dimensional random vectors on $(\Omega, \mathcal{H}, \mathbb{E})$. The following function is very important to characterize their distributions:

$$
G(p, A):=\mathbb{E}\left[\frac{1}{2}\langle A X, X\rangle+\langle p, \eta\rangle\right], \quad(p, A) \in \mathbb{R}^{d} \times \mathbb{S}(d) .
$$

It is easy to check that $G$ is a sublinear function monotonic in $A \in \mathbb{S}(d)$ in the following sense: for each $p, \bar{p} \in \mathbb{R}^{d}$ and $A, \bar{A} \in \mathbb{S}(d)$

$$
\left\{\begin{aligned}
G(p+\bar{p}, A+\bar{A}) & \leq G(p, A)+G(\bar{p}, \bar{A}) \\
G(\lambda p, \lambda A) & =\lambda G(p, A), \quad \forall \lambda \geq 0 \\
G(p, A) & \geq G(p, \bar{A}), \quad \text { if } A \geq \bar{A}
\end{aligned}\right.
$$


Clearly, $G$ is also a continuous function. By Theorem 2.1 in Chapter $[$ there exists a bounded and closed subset $\Gamma \subset \mathbb{R}^{d} \times \mathbb{R}^{d \times d}$ such that

$$
G(p, A)=\sup _{(q, Q) \in \Gamma}\left[\frac{1}{2} \operatorname{tr}\left[A Q Q^{T}\right]+\langle p, q\rangle\right] \quad \text { for }(p, A) \in \mathbb{R}^{d} \times \mathbb{S}(d) .
$$

We have the following result, which will be proved in the next section.

Proposition 1.7 Let $G: \mathbb{R}^{d} \times \mathbb{S}(d) \rightarrow \mathbb{R}$ be a given sublinear and continuous function, monotonic in $A \in \mathbb{S}(d)$ in the sense of (1.3). Then there exists a $G$ normal distributed d-dimensional random vector $X$ and a maximal distributed $d$-dimensional random vector $\eta$ on some sublinear expectation space $(\Omega, \mathcal{H}, \mathbb{E})$ satisfying (1.2) and

$$
\left(a X+b \bar{X}, a^{2} \eta+b^{2} \bar{\eta}\right) \stackrel{d}{=}\left(\sqrt{a^{2}+b^{2}} X,\left(a^{2}+b^{2}\right) \eta\right), \text { for } a, b \geq 0,
$$

where $(\bar{X}, \bar{\eta})$ is an independent copy of $(X, \eta)$.

Definition 1.8 The pair $(X, \eta)$ satisfying (1.5) is called G-distributed.

Remark 1.9 In fact, if the pair $(X, \eta)$ satisfies (1.5), then

$$
a X+b \bar{X} \stackrel{d}{=} \sqrt{a^{2}+b^{2}} X, a \eta+b \bar{\eta} \stackrel{d}{=}(a+b) \eta \text { for } a, b \geq 0 .
$$

Thus $X$ is G-normal and $\eta$ is maximal distributed.

The above pair $(X, \eta)$ is characterized by the following parabolic partial differential equation (PDE for short) defined on $[0, \infty) \times \mathbb{R}^{d} \times \mathbb{R}^{d}$ :

$$
\partial_{t} u-G\left(D_{y} u, D_{x}^{2} u\right)=0,
$$

with Cauchy condition $\left.u\right|_{t=0}=\varphi$, where $G: \mathbb{R}^{d} \times \mathbb{S}(d) \rightarrow \mathbb{R}$ is defined by (1.2) and $D^{2} u=\left(\partial_{x_{i} x_{j}}^{2} u\right)_{i, j=1}^{d}, D u=\left(\partial_{x_{i}} u\right)_{i=1}^{d}$. The PDE (1.6) is called a $G$-equation.

In this book we will mainly use the notion of viscosity solution to describe the solution of this PDE. For reader's convenience, we give a systematical introduction of the notion of viscosity solution and its related properties used in this book (see Appendix C, Section 1-3). It is worth to mention here that for the case where $G$ is non-degenerate, the viscosity solution of the $G$-equation becomes a classical $C^{1,2}$ solution (see Appendix C, Section 4). Readers without knowledge of viscosity solutions can simply understand solutions of the $G$-equation in the classical sense along the whole book.

Proposition 1.10 For the pair $(X, \eta)$ satisfying (1.5) and a function $\varphi \in$ $C_{\text {l.Lip }}\left(\mathbb{R}^{d} \times \mathbb{R}^{d}\right)$, we define

$$
u(t, x, y):=\mathbb{E}[\varphi(x+\sqrt{t} X, y+t \eta)],(t, x, y) \in[0, \infty) \times \mathbb{R}^{d} \times \mathbb{R}^{d} .
$$


Then we have

$$
u(t+s, x, y)=\mathbb{E}[u(t, x+\sqrt{s} X, y+s \eta)], \quad s \geq 0 .
$$

We also have the estimates: for each $T>0$, there exist constants $C, k>0$ such that, for all $t, s \in[0, T]$ and $x, \bar{x}, y, \bar{y} \in \mathbb{R}^{d}$,

$$
|u(t, x, y)-u(t, \bar{x}, \bar{y})| \leq C\left(1+|x|^{k}+|y|^{k}+|\bar{x}|^{k}+|\bar{y}|^{k}\right)(|x-\bar{x}|+|y-\bar{y}|)
$$

and

$$
|u(t, x, y)-u(t+s, x, y)| \leq C\left(1+|x|^{k}+|y|^{k}\right)\left(s+|s|^{1 / 2}\right) .
$$

Moreover, $u$ is the unique viscosity solution, continuous in the sense of (1.8) and (1.9), of the PDE (1.6).

Proof. Since

$$
\begin{aligned}
u(t, x, y)-u(t, \bar{x}, \bar{y})= & \mathbb{E}[\varphi(x+\sqrt{t} X, y+t \eta)]-\mathbb{E}[\varphi(\bar{x}+\sqrt{t} X, \bar{y}+t \eta)] \\
\leq & \mathbb{E}[\varphi(x+\sqrt{t} X, y+t \eta)-\varphi(\bar{x}+\sqrt{t} X, \bar{y}+t \eta)] \\
\leq & \mathbb{E}\left[C_{1}\left(1+|X|^{k}+|\eta|^{k}+|x|^{k}+|y|^{k}+|\bar{x}|^{k}+|\bar{y}|^{k}\right)\right] \\
& \times(|x-\bar{x}|+|y-\bar{y}|) \\
\leq & C\left(1+|x|^{k}+|y|^{k}+|\bar{x}|^{k}+|\bar{y}|^{k}\right)(|x-\bar{x}|+|y-\bar{y}|),
\end{aligned}
$$

we have (1.8).

Let $(\bar{X}, \bar{\eta})$ be an independent copy of $(X, \eta)$. By (1.5),

$$
\begin{aligned}
u(t+s, x, y) & =\mathbb{E}[\varphi(x+\sqrt{t+s} X, y+(t+s) \eta)] \\
& =\mathbb{E}[\varphi(x+\sqrt{s} X+\sqrt{t} \bar{X}, y+s \eta+t \bar{\eta})] \\
& =\mathbb{E}\left[\mathbb{E}[\varphi(x+\sqrt{s} \widetilde{x}+\sqrt{t} \bar{X}, y+s \widetilde{y}+t \bar{\eta})]_{(\widetilde{x}, \widetilde{y})=(X, \eta)}\right] \\
& =\mathbb{E}[u(t, x+\sqrt{s} X, y+s \eta)],
\end{aligned}
$$

we thus obtain (1.7). From this and (1.8) it follows that

$$
\begin{aligned}
& u(t+s, x, y)-u(t, x, y)=\mathbb{E}[u(t, x+\sqrt{s} X, y+s \eta)-u(t, x, y)] \\
& \leq \mathbb{E}\left[C_{1}\left(1+|x|^{k}+|y|^{k}+|X|^{k}+|\eta|^{k}\right)(\sqrt{s}|X|+s|\eta|)\right],
\end{aligned}
$$

thus we obtain (1.9).

Now, for a fixed $(t, x, y) \in(0, \infty) \times \mathbb{R}^{d} \times \mathbb{R}^{d}$, let $\psi \in C_{b}^{2,3}\left([0, \infty) \times \mathbb{R}^{d} \times \mathbb{R}^{d}\right)$ be such that $\psi \geq u$ and $\psi(t, x, y)=u(t, x, y)$. By (1.7) and Taylor's expansion, it follows that, for $\delta \in(0, t)$,

$$
\begin{aligned}
0 \leq & \mathbb{E}[\psi(t-\delta, x+\sqrt{\delta} X, y+\delta \eta)-\psi(t, x, y)] \\
\leq & \bar{C}\left(\delta^{3 / 2}+\delta^{2}\right)-\partial_{t} \psi(t, x, y) \delta \\
& +\mathbb{E}\left[\left\langle D_{x} \psi(t, x, y), X\right\rangle \sqrt{\delta}+\left\langle D_{y} \psi(t, x, y), \eta\right\rangle \delta+\frac{1}{2}\left\langle D_{x}^{2} \psi(t, x, y) X, X\right\rangle \delta\right] \\
= & -\partial_{t} \psi(t, x, y) \delta+\mathbb{E}\left[\left\langle D_{y} \psi(t, x, y), \eta\right\rangle+\frac{1}{2}\left\langle D_{x}^{2} \psi(t, x, y) X, X\right\rangle\right] \delta+\bar{C}\left(\delta^{3 / 2}+\delta^{2}\right) \\
= & -\partial_{t} \psi(t, x, y) \delta+\delta G\left(D_{y} \psi, D_{x}^{2} \psi\right)(t, x, y)+\bar{C}\left(\delta^{3 / 2}+\delta^{2}\right),
\end{aligned}
$$


from which it is easy to check that

$$
\left[\partial_{t} \psi-G\left(D_{y} \psi, D_{x}^{2} \psi\right)\right](t, x, y) \leq 0 \text {. }
$$

Thus $u$ is a viscosity subsolution of (1.6). Similarly we can prove that $u$ is a viscosity supersolution of (1.6).

Corollary 1.11 If both $(X, \eta)$ and $(\bar{X}, \bar{\eta})$ satisfy 1.5 with the same $G$, i.e.,

$G(p, A):=\mathbb{E}\left[\frac{1}{2}\langle A X, X\rangle+\langle p, \eta\rangle\right]=\mathbb{E}\left[\frac{1}{2}\langle A \bar{X}, \bar{X}\rangle+\langle p, \bar{\eta}\rangle\right] \quad$ for $(p, A) \in \mathbb{R}^{d} \times \mathbb{S}(d)$, then $(X, \eta) \stackrel{d}{=}(\bar{X}, \bar{\eta})$. In particular, $X \stackrel{d}{=}-X$.

Proof. For each $\varphi \in C_{\text {l.Lip }}\left(\mathbb{R}^{d} \times \mathbb{R}^{d}\right)$, we set

$$
\begin{aligned}
& u(t, x, y):=\mathbb{E}[\varphi(x+\sqrt{t} X, y+t \eta)], \\
& \bar{u}(t, x, y):=\mathbb{E}[\varphi(x+\sqrt{t} \bar{X}, y+t \bar{\eta})],(t, x, y) \in[0, \infty) \times \mathbb{R}^{d} \times \mathbb{R}^{d} .
\end{aligned}
$$

By Proposition 1.10, both $u$ and $\bar{u}$ are viscosity solutions of the $G$-equation (1.6) with Cauchy condition $\left.u\right|_{t=0}=\left.\bar{u}\right|_{t=0}=\varphi$. It follows from the uniqueness of the viscosity solution that $u \equiv \bar{u}$. In particular,

$$
\mathbb{E}[\varphi(X, \eta)]=\mathbb{E}[\varphi(\bar{X}, \bar{\eta})]
$$

Thus $(X, \eta) \stackrel{d}{=}(\bar{X}, \bar{\eta})$.

Corollary 1.12 Let $(X, \eta)$ satisfy (1.5). For each $\psi \in C_{l . L i p}\left(\mathbb{R}^{d}\right)$ we define

$$
v(t, x):=\mathbb{E}\left[\psi((x+\sqrt{t} X+t \eta)],(t, x) \in[0, \infty) \times \mathbb{R}^{d} .\right.
$$

Then $v$ is the unique viscosity solution of the following parabolic PDE:

$$
\partial_{t} v-G\left(D_{x} v, D_{x}^{2} v\right)=0,\left.\quad v\right|_{t=0}=\psi
$$

Moreover, we have $v(t, x+y) \equiv u(t, x, y)$, where $u$ is the solution of the PDE (1.6) with initial condition $\left.u(t, x, y)\right|_{t=0}=\psi(x+y)$.

Example 1.13 Let $X$ be $G$-normal distributed. The distribution of $X$ is characterized by

$$
u(t, x)=\mathbb{E}[\varphi(x+\sqrt{t} X)], \quad \varphi \in C_{l . L i p}\left(\mathbb{R}^{d}\right) .
$$

In particular, $\mathbb{E}[\varphi(X)]=u(1,0)$, where $u$ is the solution of the following parabolic $P D E$ defined on $[0, \infty) \times \mathbb{R}^{d}$ :

$$
\partial_{t} u-G\left(D^{2} u\right)=0,\left.\quad u\right|_{t=0}=\varphi
$$

where $G=G_{X}(A): \mathbb{S}(d) \rightarrow \mathbb{R}$ is defined by

$$
G(A):=\frac{1}{2} \mathbb{E}[\langle A X, X\rangle], \quad A \in \mathbb{S}(d) .
$$


The parabolic PDE (1.12) is called a G-heat equation.

It is easy to check that $G$ is a sublinear function defined on $\mathbb{S}(d)$. By Theorem

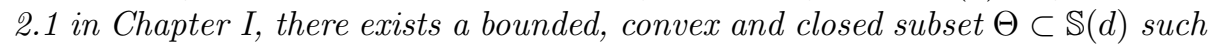
that

$$
\frac{1}{2} \mathbb{E}[\langle A X, X\rangle]=G(A)=\frac{1}{2} \sup _{Q \in \Theta} \operatorname{tr}[A Q], \quad A \in \mathbb{S}(d) .
$$

Since $G(A)$ is monotonic: $G\left(A_{1}\right) \geq G\left(A_{2}\right)$, for $A_{1} \geq A_{2}$, it follows that

$$
\Theta \subset \mathbb{S}_{+}(d)=\{\theta \in \mathbb{S}(d): \theta \geq 0\}=\left\{B B^{T}: B \in \mathbb{R}^{d \times d}\right\},
$$

where $\mathbb{R}^{d \times d}$ is the set of all $d \times d$ matrices. If $\Theta$ is a singleton: $\Theta=\{Q\}$, then $X$ is classical zero-mean normal distributed with covariance $Q$. In general, $\Theta$ characterizes the covariance uncertainty of $X$. We denote $X \stackrel{d}{=} N(\{0\} \times \Theta)$ (Recall equation (1.4), we can set $(q, Q) \in\{0\} \times \Theta)$.

When $d=1$, we have $X \stackrel{d}{=} N\left(\{0\} \times\left[\underline{\sigma}^{2}, \bar{\sigma}^{2}\right]\right.$ ) (We also denoted by $X \stackrel{d}{=}$ $\left.N\left(0,\left[\underline{\sigma}^{2}, \bar{\sigma}^{2}\right]\right)\right)$, where $\bar{\sigma}^{2}=\mathbb{E}\left[X^{2}\right]$ and $\underline{\sigma}^{2}=-\mathbb{E}\left[-X^{2}\right]$. The corresponding $G$ heat equation is

$$
\partial_{t} u-\frac{1}{2}\left(\bar{\sigma}^{2}\left(\partial_{x x}^{2} u\right)^{+}-\underline{\sigma}^{2}\left(\partial_{x x}^{2} u\right)^{-}\right)=0,\left.u\right|_{t=0}=\varphi .
$$

For the case $\underline{\sigma}^{2}>0$, this equation is also called the Barenblatt equation.

In the following two typical situations, the calculation of $\mathbb{E}[\varphi(X)]$ is very easy:

- For each convex function $\varphi$, we have

$$
\mathbb{E}[\varphi(X)]=\frac{1}{\sqrt{2 \pi}} \int_{-\infty}^{\infty} \varphi\left(\bar{\sigma}^{2} y\right) \exp \left(-\frac{y^{2}}{2}\right) d y .
$$

Indeed, for each fixed $t \geq 0$, it is easy to check that the function $u(t, x):=$ $\mathbb{E}[\varphi(x+\sqrt{t} X)]$ is convex in $x$ :

$$
\begin{aligned}
u(t, \alpha x+(1-\alpha) y) & =\mathbb{E}[\varphi(\alpha x+(1-\alpha) y+\sqrt{t} X)] \\
& \leq \alpha \mathbb{E}[\varphi(x+\sqrt{t} X)]+(1-\alpha) \mathbb{E}[\varphi(x+\sqrt{t} X)] \\
& =\alpha u(t, x)+(1-\alpha) u(t, x) .
\end{aligned}
$$

It follows that $\left(\partial_{x x}^{2} u\right)^{-} \equiv 0$ and thus the above $G$-heat equation becomes

$$
\partial_{t} u=\frac{\bar{\sigma}^{2}}{2} \partial_{x x}^{2} u,\left.\quad u\right|_{t=0}=\varphi .
$$

- For each concave function $\varphi$, we have

$$
\mathbb{E}[\varphi(X)]=\frac{1}{\sqrt{2 \pi}} \int_{-\infty}^{\infty} \varphi\left(\underline{\sigma}^{2} y\right) \exp \left(-\frac{y^{2}}{2}\right) d y .
$$


In particular,

$$
\mathbb{E}[X]=\mathbb{E}[-X]=0, \quad \mathbb{E}\left[X^{2}\right]=\bar{\sigma}^{2}, \quad-\mathbb{E}\left[-X^{2}\right]=\underline{\sigma}^{2}
$$

and

$$
\mathbb{E}\left[X^{4}\right]=3 \bar{\sigma}^{4},-\mathbb{E}\left[-X^{4}\right]=3 \underline{\sigma}^{4} .
$$

Example 1.14 Let $\eta$ be maximal distributed, the distribution of $\eta$ is characterized by the following parabolic PDE defined on $[0, \infty) \times \mathbb{R}^{d}$ :

$$
\partial_{t} u-g(D u)=0,\left.\quad u\right|_{t=0}=\varphi,
$$

where $g=g_{\eta}(p): \mathbb{R}^{d} \rightarrow \mathbb{R}$ is defined by

$$
g_{\eta}(p):=\mathbb{E}[\langle p, \eta\rangle], \quad p \in \mathbb{R}^{d} .
$$

It is easy to check that $g_{\eta}$ is a sublinear function defined on $\mathbb{R}^{d}$. By Theorem 2.1 in Chapter [, there exists a bounded, convex and closed subset $\bar{\Theta} \subset R^{d}$ such that

$$
g(p)=\sup _{q \in \bar{\Theta}}\langle p, q\rangle, \quad p \in \mathbb{R}^{d} .
$$

By this characterization, we can prove that the distribution of $\eta$ is given by

$$
\hat{\mathbb{F}}_{\eta}[\varphi]=\mathbb{E}[\varphi(\eta)]=\sup _{v \in \bar{\Theta}} \varphi(v)=\sup _{v \in \bar{\Theta}} \int_{\mathbb{R}^{d}} \varphi(x) \delta_{v}(d x), \quad \varphi \in C_{l . L i p}\left(\mathbb{R}^{d}\right),
$$

where $\delta_{v}$ is Dirac measure. Namely it is the maximal distribution with the uncertainty subset of probabilities as Dirac measures concentrated at $\bar{\Theta}$. We denote $\eta \stackrel{d}{=} N(\bar{\Theta} \times\{0\})$ (Recall equation (1.4), we can set $(q, Q) \in \bar{\Theta} \times\{0\})$.

In particular, for $d=1$,

$$
g_{\eta}(p):=\mathbb{E}[p \eta]=\bar{\mu} p^{+}-\underline{\mu} p^{-}, \quad p \in \mathbb{R},
$$

where $\bar{\mu}=\mathbb{E}[\eta]$ and $\underline{\mu}=-\hat{\mathbb{E}}[-\eta]$. The distribution of $\eta$ is given by (1.16). We denote $\eta \stackrel{d}{=} N([\underline{\mu}, \bar{\mu}] \times\{0\})$.

Exercise 1.15 We consider $X=\left(X_{1}, X_{2}\right)$, where $X_{1} \stackrel{d}{=} N\left(\{0\} \times\left[\underline{\sigma}^{2}, \bar{\sigma}^{2}\right]\right)$ with $\bar{\sigma}>\underline{\sigma}, X_{2}$ is an independent copy of $X_{1}$. Show that

(1) For each $a \in \mathbb{R}^{2},\langle a, X\rangle$ is a 1-dimensional $G$-normal distributed random variable.

(2) $X$ is not $G$-normal distributed.

Exercise 1.16 Let $X$ be G-normal distributed. For each $\varphi \in C_{\text {l.Lip }}\left(\mathbb{R}^{d}\right)$, we define a function

$$
u(t, x):=\mathbb{E}[\varphi(x+\sqrt{t} X)], \quad(t, x) \in[0, \infty) \times \mathbb{R}^{d} .
$$

Show that $u$ is the unique viscosity solution of the PDE (1.12) with Cauchy condition $\left.u\right|_{t=0}=\varphi$. 
Exercise 1.17 Let $\eta$ be maximal distributed. For each $\varphi \in C_{l . L i p}\left(\mathbb{R}^{d}\right)$, we define a function

$$
u(t, y):=\mathbb{E}[\varphi(y+t \eta)],(t, y) \in[0, \infty) \times \mathbb{R}^{d} .
$$

Show that $u$ is the unique viscosity solution of the PDE (1.14) with Cauchy condition $\left.u\right|_{t=0}=\varphi$.

\section{$\S 2$ Existence of $G$-distributed Random Variables}

In this section, we give the proof of the existence of G-distributed random variables, namely, the proof of Proposition 1.7

Let $G: \mathbb{R}^{d} \times \mathbb{S}(d) \rightarrow \mathbb{R}$ be a given sublinear function monotonic in $A \in \mathbb{S}(d)$ in the sense of (1.3). We now construct a pair of $d$-dimensional random vectors $(X, \eta)$ on some sublinear expectation space $(\Omega, \mathcal{H}, \mathbb{E})$ satisfying (1.2) and (1.5).

For each $\varphi \in C_{l . L i p}\left(\mathbb{R}^{2 d}\right)$, let $u=u^{\varphi}$ be the unique viscosity solution of the $G$-equation (1.6) with $\left.u^{\varphi}\right|_{t=0}=\varphi$. We take $\widetilde{\Omega}=\mathbb{R}^{2 d}, \widetilde{\mathcal{H}}=C_{\text {l.Lip }}\left(\mathbb{R}^{2 d}\right)$ and $\widetilde{\omega}=(x, y) \in \mathbb{R}^{2 d}$. The corresponding sublinear expectation $\widetilde{\mathbb{E}}[\cdot]$ is defined by $\widetilde{\mathbb{E}}[\xi]=u^{\varphi}(1,0,0)$, for each $\xi \in \widetilde{\mathcal{H}}$ of the form $\xi(\widetilde{\omega})=(\varphi(x, y))_{(x, y) \in \mathbb{R}^{2 d}} \in$ $C_{\text {l.Lip }}\left(\mathbb{R}^{2 d}\right)$. The monotonicity and sub-additivity of $u^{\varphi}$ with respect to $\varphi$ are known in the theory of viscosity solution. For reader's convenience we provide a new and simple proof in Appendix C (see Corollary 2.4 and Corollary 2.5). The constant preserving and positive homogeneity of $\widetilde{\mathbb{E}}[\cdot]$ are easy to check. Thus the functional $\widetilde{\mathbb{E}}[\cdot]: \widetilde{\mathcal{H}} \rightarrow \mathbb{R}$ forms a sublinear expectation.

We now consider a pair of $d$-dimensional random vectors $(\widetilde{X}, \widetilde{\eta})(\widetilde{\omega})=(x, y)$. We have

$$
\widetilde{\mathbb{E}}[\varphi(\tilde{X}, \widetilde{\eta})]=u^{\varphi}(1,0,0) \quad \text { for } \varphi \in C_{l . L i p}\left(\mathbb{R}^{2 d}\right) .
$$

In particular, just setting $\varphi_{0}(x, y)=\frac{1}{2}\langle A x, x\rangle+\langle p, y\rangle$, we can check that

$$
u^{\varphi_{0}}(t, x, y)=G(p, A) t+\frac{1}{2}\langle A x, x\rangle+\langle p, y\rangle .
$$

We thus have

$$
\widetilde{\mathbb{E}}\left[\frac{1}{2}\langle A \widetilde{X}, \widetilde{X}\rangle+\langle p, \widetilde{\eta}\rangle\right]=u^{\varphi_{0}}(1,0,0)=G(p, A), \quad(p, A) \in \mathbb{R}^{d} \times \mathbb{S}(d) .
$$

We construct a product space

$$
(\Omega, \mathcal{H}, \mathbb{E})=(\widetilde{\Omega} \times \widetilde{\Omega}, \widetilde{\mathcal{H}} \otimes \widetilde{\mathcal{H}}, \widetilde{\mathbb{E}} \otimes \widetilde{\mathbb{E}}),
$$

and introduce two pairs of random vectors

$$
(X, \eta)\left(\widetilde{\omega}_{1}, \widetilde{\omega}_{2}\right)=\widetilde{\omega}_{1},(\bar{X}, \bar{\eta})\left(\widetilde{\omega}_{1}, \widetilde{\omega}_{2}\right)=\widetilde{\omega}_{2}, \quad\left(\widetilde{\omega}_{1}, \widetilde{\omega}_{2}\right) \in \widetilde{\Omega} \times \widetilde{\Omega} .
$$

By Proposition 3.15 in Chapter $\llbracket(X, \eta) \stackrel{d}{=}(\widetilde{X}, \widetilde{\eta})$ and $(\bar{X}, \bar{\eta})$ is an independent copy of $(X, \eta)$. 
We now prove that the distribution of $(X, \eta)$ satisfies condition (1.5). For each $\varphi \in C_{l \text {.Lip }}\left(\mathbb{R}^{2 d}\right)$ and for each fixed $\lambda>0,(\bar{x}, \bar{y}) \in \mathbb{R}^{2 d}$, since the function $v$ defined by $v(t, x, y):=u^{\varphi}(\lambda t, \bar{x}+\sqrt{\lambda} x, \bar{y}+\lambda y)$ solves exactly the same equation (1.6), but with Cauchy condition

$$
\left.v\right|_{t=0}=\varphi(\bar{x}+\sqrt{\lambda} \times \cdot, \bar{y}+\lambda \times \cdot) .
$$

Thus

$$
\mathbb{E}[\varphi(\bar{x}+\sqrt{\lambda} X, \bar{y}+\lambda \eta)]=v(1,0,0)=u^{\varphi}(\lambda, \bar{x}, \bar{y}) .
$$

By the definition of $\mathbb{E}$, for each $t>0$ and $s>0$,

$$
\begin{aligned}
\mathbb{E}[\varphi(\sqrt{t} X+\sqrt{s} \bar{X}, t \eta+s \bar{\eta})] & =\mathbb{E}\left[\mathbb{E}[\varphi(\sqrt{t} x+\sqrt{s} \bar{X}, t y+s \bar{\eta})]_{(x, y)=(X, \eta)}\right] \\
& =\mathbb{E}\left[u^{\varphi}(s, \sqrt{t} X, t \eta)\right]=u^{u^{\varphi}(s, \cdot, \cdot)}(t, 0,0) \\
& =u^{\varphi}(t+s, 0,0) \\
& =\mathbb{E}[\varphi(\sqrt{t+s} X,(t+s) \eta)] .
\end{aligned}
$$

Namely $(\sqrt{t} X+\sqrt{s} \bar{X}, t \eta+s \bar{\eta}) \stackrel{d}{=}(\sqrt{t+s} X,(t+s) \eta)$. Thus the distribution of $(X, \eta)$ satisfies condition (1.5).

Remark 2.1 From now on, when we mention the sublinear expectation space $(\Omega, \mathcal{H}, \mathbb{E})$, we suppose that there exists a pair of random vectors $(X, \eta)$ on $(\Omega, \mathcal{H}, \mathbb{E})$ such that $(X, \eta)$ is $G$-distributed.

Exercise 2.2 Prove that $\mathbb{E}\left[X^{3}\right]>0$ for $X \stackrel{d}{=} N\left(\{0\} \times\left[\underline{\sigma}^{2}, \bar{\sigma}^{2}\right]\right)$ with $\underline{\sigma}^{2}<\bar{\sigma}^{2}$.

It is worth to point that $\mathbb{E}[\varphi(X)]$ not always equal to $\sup _{\underline{\sigma}^{2} \leq \sigma \leq \bar{\sigma}^{2}} E_{\sigma}[\varphi(X)]$ for $\varphi \in C_{l, L i p}(\mathbb{R})$, where $E_{\sigma}$ denotes the linear expectation corresponding to the normal distributed density function $N\left(0, \sigma^{2}\right)$.

\section{$\S 3$ Law of Large Numbers and Central Limit Theorem}

Theorem 3.1 (Law of large numbers) Let $\left\{Y_{i}\right\}_{i=1}^{\infty}$ be a sequence of $\mathbb{R}^{d}$ valued random variables on a sublinear expectation space $(\Omega, \mathcal{H}, \mathbb{E})$. We assume that $Y_{i+1} \stackrel{d}{=} Y_{i}$ and $Y_{i+1}$ is independent from $\left\{Y_{1}, \cdots, Y_{i}\right\}$ for each $i=1,2, \cdots$. Then the sequence $\left\{\bar{S}_{n}\right\}_{n=1}^{\infty}$ defined by

$$
\bar{S}_{n}:=\frac{1}{n} \sum_{i=1}^{n} Y_{i}
$$

converges in law to a maximal distribution, i.e.,

$$
\lim _{n \rightarrow \infty} \mathbb{E}\left[\varphi\left(\bar{S}_{n}\right)\right]=\mathbb{E}[\varphi(\eta)]
$$


for all functions $\varphi \in C\left(\mathbb{R}^{d}\right)$ satisfying linear growth condition $(|\varphi(x)| \leq C(1+$ $|x|)$ ), where $\eta$ is a maximal distributed random vector and the corresponding sublinear function $g: \mathbb{R}^{d} \rightarrow \mathbb{R}$ is defined by

$$
g(p):=\mathbb{E}\left[\left\langle p, Y_{1}\right\rangle\right], \quad p \in \mathbb{R}^{d} .
$$

Remark 3.2 When $d=1$, the sequence $\left\{\bar{S}_{n}\right\}_{n=1}^{\infty}$ converges in law to $N([\underline{\mu}, \bar{\mu}] \times$ $\{0\})$, where $\bar{\mu}=\mathbb{E}\left[Y_{1}\right]$ and $\underline{\mu}=-\mathbb{E}\left[-Y_{1}\right]$. For the general case, the sum $\frac{1}{n} \sum_{i=1}^{n} Y_{i}$ converges in law to $N(\bar{\Theta} \times\{0\})$, where $\bar{\Theta} \subset \mathbb{R}^{d}$ is the bounded, convex and closed subset defined in Example 1.14. If we take in particular $\varphi(y)=d_{\bar{\Theta}}(y)=\inf \{|x-y|: x \in \bar{\Theta}\}$, then by (3.17) we have the following generalized law of large numbers:

$$
\lim _{n \rightarrow \infty} \mathbb{E}\left[d_{\bar{\Theta}}\left(\frac{1}{n} \sum_{i=1}^{n} Y_{i}\right)\right]=\sup _{\theta \in \bar{\Theta}} d_{\bar{\Theta}}(\theta)=0 .
$$

If $Y_{i}$ has no mean-uncertainty, or in other words, $\bar{\Theta}$ is a singleton: $\bar{\Theta}=\{\bar{\theta}\}$, then (3.18) becomes

$$
\lim _{n \rightarrow \infty} \mathbb{E}\left[\left|\frac{1}{n} \sum_{i=1}^{n} Y_{i}-\bar{\theta}\right|\right]=0 .
$$

Theorem 3.3 (Central limit theorem with zero-mean) Let $\left\{X_{i}\right\}_{i=1}^{\infty}$ be a sequence of $\mathbb{R}^{d}$-valued random variables on a sublinear expectation space $(\Omega, \mathcal{H}, \mathbb{E})$. We assume that $X_{i+1} \stackrel{d}{=} X_{i}$ and $X_{i+1}$ is independent from $\left\{X_{1}, \cdots, X_{i}\right\}$ for each $i=1,2, \cdots$. We further assume that

$$
\mathbb{E}\left[X_{1}\right]=\mathbb{E}\left[-X_{1}\right]=0 .
$$

Then the sequence $\left\{\bar{S}_{n}\right\}_{n=1}^{\infty}$ defined by

$$
\bar{S}_{n}:=\frac{1}{\sqrt{n}} \sum_{i=1}^{n} X_{i}
$$

converges in law to $X$, i.e.,

$$
\lim _{n \rightarrow \infty} \mathbb{E}\left[\varphi\left(\bar{S}_{n}\right)\right]=\mathbb{E}[\varphi(X)],
$$

for all functions $\varphi \in C\left(\mathbb{R}^{d}\right)$ satisfying linear growth condition, where $X$ is a $G$-normal distributed random vector and the corresponding sublinear function $G: \mathbb{S}(d) \rightarrow \mathbb{R}$ is defined by

$$
G(A):=\mathbb{E}\left[\frac{1}{2}\left\langle A X_{1}, X_{1}\right\rangle\right], \quad A \in \mathbb{S}(d) .
$$

Remark 3.4 When $d=1$, the sequence $\left\{\bar{S}_{n}\right\}_{n=1}^{\infty}$ converges in law to $N(\{0\} \times$ $\left.\left[\underline{\sigma}^{2}, \bar{\sigma}^{2}\right]\right)$, where $\bar{\sigma}^{2}=\mathbb{E}\left[X_{1}^{2}\right]$ and $\underline{\sigma}^{2}=-\mathbb{E}\left[-X_{1}^{2}\right]$. In particular, if $\bar{\sigma}^{2}=\underline{\sigma}^{2}$, then it becomes a classical central limit theorem. 
The following theorem is a nontrivial generalization of the above two theorems.

Theorem 3.5 (Central limit theorem with law of large numbers) Let $\left\{\left(X_{i}, Y_{i}\right)\right\}_{i=1}^{\infty}$ be a sequence of $\mathbb{R}^{d} \times \mathbb{R}^{d}$-valued random vectors on a sublinear expectation space $(\Omega, \mathcal{H}, \mathbb{E})$. We assume that $\left(X_{i+1}, Y_{i+1}\right) \stackrel{d}{=}\left(X_{i}, Y_{i}\right)$ and $\left(X_{i+1}, Y_{i+1}\right)$ is independent from $\left\{\left(X_{1}, Y_{1}\right), \cdots,\left(X_{i}, Y_{i}\right)\right\}$ for each $i=1,2, \cdots$. We further assume that

$$
\mathbb{E}\left[X_{1}\right]=\mathbb{E}\left[-X_{1}\right]=0 \text {. }
$$

Then the sequence $\left\{\bar{S}_{n}\right\}_{n=1}^{\infty}$ defined by

$$
\bar{S}_{n}:=\sum_{i=1}^{n}\left(\frac{X_{i}}{\sqrt{n}}+\frac{Y_{i}}{n}\right)
$$

converges in law to $X+\eta$, i.e.,

$$
\lim _{n \rightarrow \infty} \mathbb{E}\left[\varphi\left(\bar{S}_{n}\right)\right]=\mathbb{E}[\varphi(X+\eta)]
$$

for all functions $\varphi \in C\left(\mathbb{R}^{d}\right)$ satisfying a linear growth condition, where the pair $(X, \eta)$ is $G$-distributed. The corresponding sublinear function $G: \mathbb{R}^{d} \times \mathbb{S}(d) \rightarrow \mathbb{R}$ is defined by

$$
G(p, A):=\mathbb{E}\left[\left\langle p, Y_{1}\right\rangle+\frac{1}{2}\left\langle A X_{1}, X_{1}\right\rangle\right], \quad A \in \mathbb{S}(d), \quad p \in \mathbb{R}^{d} .
$$

Thus $\mathbb{E}[\varphi(X+\eta)]$ can be calculated by Corollary 1.12 .

The following result is equivalent to the above central limit theorem.

Theorem 3.6 We make the same assumptions as in Theorem 3.5. Then for each function $\varphi \in C\left(\mathbb{R}^{d} \times \mathbb{R}^{d}\right)$ satisfying linear growth condition, we have

$$
\lim _{n \rightarrow \infty} \mathbb{E}\left[\varphi\left(\sum_{i=1}^{n} \frac{X_{i}}{\sqrt{n}}, \sum_{i=1}^{n} \frac{Y_{i}}{n}\right)\right]=\mathbb{E}[\varphi(X, \eta)]
$$

Proof. It is easy to prove Theorem 3.5 by Theorem 3.6 . To prove Theorem 3.6 from Theorem 3.5. it suffices to define a pair of $2 d$-dimensional random vectors

$$
\bar{X}_{i}=\left(X_{i}, 0\right), \quad \bar{Y}_{i}=\left(0, Y_{i}\right) \text { for } i=1,2, \cdots .
$$

We have

$$
\begin{aligned}
\lim _{n \rightarrow \infty} \mathbb{E}\left[\varphi\left(\sum_{i=1}^{n} \frac{X_{i}}{\sqrt{n}}, \sum_{i=1}^{n} \frac{Y_{i}}{n}\right)\right] & =\lim _{n \rightarrow \infty} \mathbb{E}\left[\varphi\left(\sum_{i=1}^{n}\left(\frac{\bar{X}_{i}}{\sqrt{n}}+\frac{\bar{Y}_{i}}{n}\right)\right)\right]=\mathbb{E}[\varphi(\bar{X}+\eta)] \\
& =\mathbb{E}[\varphi(X, \eta)]
\end{aligned}
$$

with $\bar{X}=(X, 0)$ and $\bar{\eta}=(0, \eta)$. 
To prove Theorem 3.5. we need the following norms to measure the regularity of a given real functions $u$ defined on $Q=[0, T] \times \mathbb{R}^{d}$ :

$$
\begin{aligned}
\|u\|_{C^{0,0}(Q)} & =\sup _{(t, x) \in Q}|u(t, x)| \\
\|u\|_{C^{1,1}(Q)} & =\|u\|_{C^{0,0}(Q)}+\left\|\partial_{t} u\right\|_{C^{0,0}(Q)}+\sum_{i=1}^{d}\left\|\partial_{x_{i}} u\right\|_{C^{0,0}(Q)}, \\
\|u\|_{C^{1,2}(Q)} & =\|u\|_{C^{1,1}(Q)}+\sum_{i, j=1}^{d}\left\|\partial_{x_{i} x_{j}} u\right\|_{C^{0,0}(Q)} .
\end{aligned}
$$

For given constants $\alpha, \beta \in(0,1)$, we denote

$$
\begin{aligned}
\|u\|_{C^{\alpha, \beta}(Q)} & =\sup _{\substack{x, y \in \mathbb{R}^{d}, x \neq y \\
s, t \in[0, T], s \neq t}} \frac{|u(s, x)-u(t, y)|}{|r-s|^{\alpha}+|x-y|^{\beta}}, \\
\|u\|_{C^{1+\alpha, 1+\beta}(Q)} & =\|u\|_{C^{\alpha, \beta}(Q)}+\left\|\partial_{t} u\right\|_{C^{\alpha, \beta}(Q)}+\sum_{i=1}^{d}\left\|\partial_{x_{i}} u\right\|_{C^{\alpha, \beta}(Q)}, \\
\|u\|_{C^{1+\alpha, 2+\beta}(Q)} & =\|u\|_{C^{1+\alpha, 1+\beta}(Q)}+\sum_{i, j=1}^{d}\left\|\partial_{x_{i} x_{j}} u\right\|_{C^{\alpha, \beta}(Q)} .
\end{aligned}
$$

If, for example, $\|u\|_{C^{1+\alpha, 2+\beta}(Q)}<\infty$, then $u$ is said to be a $C^{1+\alpha, 2+\beta}$-function on $Q$.

We need the following lemma.

Lemma 3.7 We assume the same assumptions as in Theorem 3.5. We further assume that there exists a constant $\beta>0$ such that, for each $A, \bar{A} \in \mathbb{S}(d)$ with $A \geq \bar{A}$, we have

$$
\mathbb{E}\left[\left\langle A X_{1}, X_{1}\right\rangle\right]-\mathbb{E}\left[\left\langle\bar{A} X_{1}, X_{1}\right\rangle\right] \geq \beta \operatorname{tr}[A-\bar{A}] .
$$

Then our main result (3.19) holds.

Proof. We first prove (3.19) for $\varphi \in C_{b . L i p}\left(\mathbb{R}^{d}\right)$. For a small but fixed $h>0$, let $V$ be the unique viscosity solution of

$$
\partial_{t} V+G\left(D V, D^{2} V\right)=0,(t, x) \in[0,1+h) \times \mathbb{R}^{d},\left.\quad V\right|_{t=1+h}=\varphi .
$$

Since $(X, \eta)$ satisfies (1.5), we have

$$
V(h, 0)=\mathbb{E}[\varphi(X+\eta)], \quad V(1+h, x)=\varphi(x) .
$$

Since (3.21) is a uniformly parabolic PDE and $G$ is a convex function, by the interior regularity of $V$ (see Appendix $\mathrm{C}$ ), we have

$$
\|V\|_{C^{1+\alpha / 2,2+\alpha}\left([0,1] \times \mathbb{R}^{d}\right)}<\infty \text { for some } \alpha \in(0,1) .
$$


We set $\delta=\frac{1}{n}$ and $S_{0}=0$. Then

$$
\begin{aligned}
& V\left(1, \bar{S}_{n}\right)-V(0,0)=\sum_{i=0}^{n-1}\left\{V\left((i+1) \delta, \bar{S}_{i+1}\right)-V\left(i \delta, \bar{S}_{i}\right)\right\} \\
& =\sum_{i=0}^{n-1}\left\{\left[V\left((i+1) \delta, \bar{S}_{i+1}\right)-V\left(i \delta, \bar{S}_{i+1}\right)\right]+\left[V\left(i \delta, \bar{S}_{i+1}\right)-V\left(i \delta, \bar{S}_{i}\right)\right]\right\} \\
& =\sum_{i=0}^{n-1}\left\{I_{\delta}^{i}+J_{\delta}^{i}\right\}
\end{aligned}
$$

with, by Taylor's expansion,

$$
\begin{aligned}
J_{\delta}^{i}= & \partial_{t} V\left(i \delta, \bar{S}_{i}\right) \delta+\frac{1}{2}\left\langle D^{2} V\left(i \delta, \bar{S}_{i}\right) X_{i+1}, X_{i+1}\right\rangle \delta+\left\langle D V\left(i \delta, \bar{S}_{i}\right), X_{i+1} \sqrt{\delta}+Y_{i+1} \delta\right\rangle \\
I_{\delta}^{i}= & \delta \int_{0}^{1}\left[\partial_{t} V\left((i+\beta) \delta, \bar{S}_{i+1}\right)-\partial_{t} V\left(i \delta, \bar{S}_{i+1}\right)\right] d \beta+\left[\partial_{t} V\left(i \delta, \bar{S}_{i+1}\right)-\partial_{t} V\left(i \delta, \bar{S}_{i}\right)\right] \delta \\
& +\left\langle D^{2} V\left(i \delta, \bar{S}_{i}\right) X_{i+1}, Y_{i+1}\right\rangle \delta^{3 / 2}+\frac{1}{2}\left\langle D^{2} V\left(i \delta, \bar{S}_{i}\right) Y_{i+1}, Y_{i+1}\right\rangle \delta^{2} \\
& +\int_{0}^{1} \int_{0}^{1}\left\langle\Theta_{\beta \gamma}^{i}\left(X_{i+1} \sqrt{\delta}+Y_{i+1} \delta\right), X_{i+1} \sqrt{\delta}+Y_{i+1} \delta\right\rangle \gamma d \beta d \gamma
\end{aligned}
$$

with

$$
\Theta_{\beta \gamma}^{i}=D^{2} V\left(i \delta, \bar{S}_{i}+\gamma \beta\left(X_{i+1} \sqrt{\delta}+Y_{i+1} \delta\right)\right)-D^{2} V\left(i \delta, \bar{S}_{i}\right) .
$$

Thus

$$
\mathbb{E}\left[\sum_{i=0}^{n-1} J_{\delta}^{i}\right]-\mathbb{E}\left[-\sum_{i=0}^{n-1} I_{\delta}^{i}\right] \leq \mathbb{E}\left[V\left(1, \bar{S}_{n}\right)\right]-V(0,0) \leq \mathbb{E}\left[\sum_{i=0}^{n-1} J_{\delta}^{i}\right]+\mathbb{E}\left[\sum_{i=0}^{n-1} I_{\delta}^{i}\right] .
$$

We now prove that $\mathbb{E}\left[\sum_{i=0}^{n-1} J_{\delta}^{i}\right]=0$. For $J_{\delta}^{i}$, note that

$$
\mathbb{E}\left[\left\langle D V\left(i \delta, \bar{S}_{i}\right), X_{i+1} \sqrt{\delta}\right\rangle\right]=\mathbb{E}\left[-\left\langle D V\left(i \delta, \bar{S}_{i}\right), X_{i+1} \sqrt{\delta}\right\rangle\right]=0,
$$

then, from the definition of the function $G$, we have

$$
\mathbb{E}\left[J_{\delta}^{i}\right]=\mathbb{E}\left[\partial_{t} V\left(i \delta, \bar{S}_{i}\right)+G\left(D V\left(i \delta, \bar{S}_{i}\right), D^{2} V\left(i \delta, \bar{S}_{i}\right)\right)\right] \delta .
$$

Combining the above two equalities with $\partial_{t} V+G\left(D V, D^{2} V\right)=0$ as well as the independence of $\left(X_{i+1}, Y_{i+1}\right)$ from $\left\{\left(X_{1}, Y_{1}\right), \cdots,\left(X_{i}, Y_{i}\right)\right\}$, it follows that

$$
\mathbb{E}\left[\sum_{i=0}^{n-1} J_{\delta}^{i}\right]=\mathbb{E}\left[\sum_{i=0}^{n-2} J_{\delta}^{i}\right]=\cdots=0
$$

Thus (3.23) can be rewritten as

$$
-\mathbb{E}\left[-\sum_{i=0}^{n-1} I_{\delta}^{i}\right] \leq \mathbb{E}\left[V\left(1, \bar{S}_{n}\right)\right]-V(0,0) \leq \mathbb{E}\left[\sum_{i=0}^{n-1} I_{\delta}^{i}\right] .
$$


But since both $\partial_{t} V$ and $D^{2} V$ are uniformly $\frac{\alpha}{2}$-hölder continuous in $t$ and $\alpha$ hölder continuous in $x$ on $[0,1] \times \mathbb{R}^{d}$, we then have

$$
\left|I_{\delta}^{i}\right| \leq C \delta^{1+\alpha / 2}\left(1+\left|X_{i+1}\right|^{2+\alpha}+\left|Y_{i+1}\right|^{2+\alpha}\right) .
$$

It follows that

$$
\mathbb{E}\left[\left|I_{\delta}^{i}\right|\right] \leq C \delta^{1+\alpha / 2}\left(1+\mathbb{E}\left[\left|X_{1}\right|^{2+\alpha}+\left|Y_{1}\right|^{2+\alpha}\right]\right) .
$$

Thus

$$
\begin{aligned}
-C\left(\frac{1}{n}\right)^{\alpha / 2}\left(1+\mathbb{E}\left[\left|X_{1}\right|^{2+\alpha}+\left|Y_{1}\right|^{2+\alpha}\right]\right) & \leq \mathbb{E}\left[V\left(1, \bar{S}_{n}\right)\right]-V(0,0) \\
& \leq C\left(\frac{1}{n}\right)^{\alpha / 2}\left(1+\mathbb{E}\left[\left|X_{1}\right|^{2+\alpha}+\left|Y_{1}\right|^{2+\alpha}\right]\right) .
\end{aligned}
$$

As $n \rightarrow \infty$, we have

$$
\lim _{n \rightarrow \infty} \mathbb{E}\left[V\left(1, \bar{S}_{n}\right)\right]=V(0,0) .
$$

On the other hand, for each $t, t^{\prime} \in[0,1+h]$ and $x \in \mathbb{R}^{d}$, we have

$$
\left|V(t, x)-V\left(t^{\prime}, x\right)\right| \leq C\left(\sqrt{\left|t-t^{\prime}\right|}+\left|t-t^{\prime}\right|\right) .
$$

Thus $|V(0,0)-V(h, 0)| \leq C(\sqrt{h}+h)$ and, by (3.24)

$$
\left|\mathbb{E}\left[V\left(1, \bar{S}_{n}\right)\right]-\mathbb{E}\left[\varphi\left(\bar{S}_{n}\right)\right]\right|=\left|\mathbb{E}\left[V\left(1, \bar{S}_{n}\right)\right]-\mathbb{E}\left[V\left(1+h, \bar{S}_{n}\right)\right]\right| \leq C(\sqrt{h}+h) .
$$

It follows from (3.22) and (3.24) that

$$
\limsup _{n \rightarrow \infty}\left|\mathbb{E}\left[\varphi\left(\bar{S}_{n}\right)\right]-\mathbb{E}[\varphi(X+\eta)]\right| \leq 2 C(\sqrt{h}+h) .
$$

Since $h$ can be arbitrarily small, we have

$$
\lim _{n \rightarrow \infty} \mathbb{E}\left[\varphi\left(\bar{S}_{n}\right)\right]=\mathbb{E}[\varphi(X+\eta)]
$$

Remark 3.8 From the proof we can check that the main assumption of identical distribution of $\left\{X_{i}, Y_{i}\right\}_{i=1}^{\infty}$ can be weaken to

$$
\mathbb{E}\left[\left\langle p, Y_{i}\right\rangle+\frac{1}{2}\left\langle A X_{i}, X_{i}\right\rangle\right]=G(p, A), \quad i=1,2, \cdots, p \in \mathbb{R}^{d}, A \in \mathbb{S}(d) .
$$

Another essential condition is $\mathbb{E}\left[\left|X_{i}\right|^{2+\delta}\right]+\mathbb{E}\left[\left|Y_{i}\right|^{1+\delta}\right] \leq C$ for some $\delta>0$. We do not need the condition $\mathbb{E}\left[\left|X_{i}\right|^{n}\right]+\mathbb{E}\left[\left|Y_{i}\right|^{n}\right]<\infty$ for each $n \in \mathbb{N}$.

We now give the proof of Theorem 3.5 .

Proof of Theorem 3.5. For the case when the uniform elliptic condition (3.20) does not hold, we first introduce a perturbation to prove the above convergence for $\varphi \in C_{b . L i p}\left(\mathbb{R}^{d}\right)$. According to Definition 3.14 and Proposition 3.15in Chap I, 
we can construct a sublinear expectation space $(\bar{\Omega}, \overline{\mathcal{H}}, \overline{\mathbb{E}})$ and a sequence of three random vectors $\left\{\left(\bar{X}_{i}, \bar{Y}_{i}, \bar{\kappa}_{i}\right)\right\}_{i=1}^{\infty}$ such that, for each $n=1,2, \cdots,\left\{\left(\bar{X}_{i}, \bar{Y}_{i}\right)\right\}_{i=1}^{n} \stackrel{d}{=}$ $\left\{\left(X_{i}, Y_{i}\right)\right\}_{i=1}^{n}$ and $\left(\bar{X}_{n+1}, \bar{Y}_{n+1}, \bar{\kappa}_{n+1}\right)$ is independent from $\left\{\left(\bar{X}_{i}, \bar{Y}_{i}, \bar{\kappa}_{i}\right)\right\}_{i=1}^{n}$ and, moreover,

$$
\overline{\mathbb{E}}\left[\psi\left(\bar{X}_{i}, \bar{Y}_{i}, \bar{\kappa}_{i}\right)\right]=(2 \pi)^{-d / 2} \int_{\mathbb{R}^{d}} \mathbb{E}\left[\psi\left(X_{i}, Y_{i}, x\right)\right] e^{-|x|^{2} / 2} d x \text { for } \psi \in C_{l . L i p}\left(\mathbb{R}^{3 \times d}\right) .
$$

We then use the perturbation $\bar{X}_{i}^{\varepsilon}=\bar{X}_{i}+\varepsilon \bar{\kappa}_{i}$ for a fixed $\varepsilon>0$. It is easy to see that the sequence $\left\{\left(\bar{X}_{i}^{\varepsilon}, \bar{Y}_{i}\right)\right\}_{i=1}^{\infty}$ satisfies all conditions in the above CLT, in particular,

$$
G_{\varepsilon}(p, A):=\overline{\mathbb{E}}\left[\frac{1}{2}\left\langle A \bar{X}_{1}^{\varepsilon}, \bar{X}_{1}^{\varepsilon}\right\rangle+\left\langle p, \bar{Y}_{1}\right\rangle\right]=G(p, A)+\frac{\varepsilon^{2}}{2} \operatorname{tr}[A] .
$$

Thus it is strictly elliptic. We then can apply Lemma 3.7 to

$$
\bar{S}_{n}^{\varepsilon}:=\sum_{i=1}^{n}\left(\frac{\bar{X}_{i}^{\varepsilon}}{\sqrt{n}}+\frac{\bar{Y}_{i}}{n}\right)=\sum_{i=1}^{n}\left(\frac{\bar{X}_{i}}{\sqrt{n}}+\frac{\bar{Y}_{i}}{n}\right)+\varepsilon J_{n}, \quad J_{n}=\sum_{i=1}^{n} \frac{\bar{\kappa}_{i}}{\sqrt{n}}
$$

and obtain

$$
\lim _{n \rightarrow \infty} \overline{\mathbb{E}}\left[\varphi\left(\bar{S}_{n}^{\varepsilon}\right)\right]=\overline{\mathbb{E}}[\varphi(\bar{X}+\bar{\eta}+\varepsilon \bar{\kappa})],
$$

where $((\bar{X}, \bar{\kappa}),(\bar{\eta}, 0))$ is $\bar{G}$-distributed under $\overline{\mathbb{E}}[\cdot]$ and

$$
\bar{G}(\bar{p}, \bar{A}):=\overline{\mathbb{E}}\left[\frac{1}{2}\left\langle\bar{A}\left(\bar{X}_{1}, \bar{\kappa}_{1}\right)^{T},\left(\bar{X}_{1}, \bar{\kappa}_{1}\right)^{T}\right\rangle+\left\langle\bar{p},\left(\bar{Y}_{1}, 0\right)^{T}\right\rangle\right], \quad \bar{A} \in \mathbb{S}(2 d), \quad \bar{p} \in \mathbb{R}^{2 d} .
$$

By Proposition 1.6, it is easy to prove that $(\bar{X}+\varepsilon \bar{\kappa}, \bar{\eta})$ is $G_{\varepsilon}$-distributed and $(\bar{X}, \bar{\eta})$ is $G$-distributed. But we have

$$
\begin{aligned}
\left|\mathbb{E}\left[\varphi\left(\bar{S}_{n}\right)\right]-\overline{\mathbb{E}}\left[\varphi\left(\bar{S}_{n}^{\varepsilon}\right)\right]\right| & =\left|\overline{\mathbb{E}}\left[\varphi\left(\bar{S}_{n}^{\varepsilon}-\varepsilon J_{n}\right)\right]-\overline{\mathbb{E}}\left[\varphi\left(\bar{S}_{n}^{\varepsilon}\right)\right]\right| \\
& \leq \varepsilon C \overline{\mathbb{E}}\left[\left|J_{n}\right|\right] \leq C^{\prime} \varepsilon
\end{aligned}
$$

and similarly,

$|\mathbb{E}[\varphi(X+\eta)]-\overline{\mathbb{E}}[\varphi(\bar{X}+\bar{\eta}+\varepsilon \bar{\kappa})]|=|\overline{\mathbb{E}}[\varphi(\bar{X}+\bar{\eta})]-\overline{\mathbb{E}}[\varphi(\bar{X}+\bar{\eta}+\varepsilon \bar{\kappa})]| \leq C \varepsilon$. Since $\varepsilon$ can be arbitrarily small, it follows that

$$
\lim _{n \rightarrow \infty} \mathbb{E}\left[\varphi\left(\bar{S}_{n}\right)\right]=\mathbb{E}[\varphi(X+\eta)] \text { for } \varphi \in C_{b . L i p}\left(\mathbb{R}^{d}\right) .
$$

On the other hand, it is easy to check that $\sup _{n} \mathbb{E}\left[\left|\bar{S}_{n}\right|^{2}\right]+\mathbb{E}\left[|X+\eta|^{2}\right]<\infty$. We then can apply the following lemma to prove that the above convergence holds for $\varphi \in C\left(\mathbb{R}^{d}\right)$ with linear growth condition. The proof is complete.

Lemma 3.9 Let $(\Omega, \mathcal{H}, \mathbb{E})$ and $(\widetilde{\Omega}, \widetilde{\mathcal{H}}, \widetilde{\mathbb{E}})$ be two sublinear expectation spaces and let $Y_{n} \in \mathcal{H}$ and $Y \in \widetilde{\mathcal{H}}, n=1,2, \cdots$, be given. We assume that, for a given $p \geq$ $1, \sup _{n} \mathbb{E}\left[\left|Y_{n}\right|^{p}\right]+\widetilde{\mathbb{E}}\left[|Y|^{p}\right]<\infty$. If the convergence $\lim _{n \rightarrow \infty} \mathbb{E}\left[\varphi\left(Y_{n}\right)\right]=\widetilde{\mathbb{E}}[\varphi(Y)]$ holds for each $\varphi \in C_{b . L i p}\left(\mathbb{R}^{d}\right)$, then it also holds for all functions $\varphi \in C\left(\mathbb{R}^{d}\right)$ with the growth condition $|\varphi(x)| \leq C\left(1+|x|^{p-1}\right)$. 
Proof. We first prove that the above convergence holds for $\varphi \in C_{b}\left(\mathbb{R}^{d}\right)$ with a compact support. In this case, for each $\varepsilon>0$, we can find a $\bar{\varphi} \in C_{b . L i p}\left(\mathbb{R}^{d}\right)$ such that $\sup _{x \in \mathbb{R}^{d}}|\varphi(x)-\bar{\varphi}(x)| \leq \frac{\varepsilon}{2}$. We have

$$
\begin{aligned}
& \left|\mathbb{E}\left[\varphi\left(Y_{n}\right)\right]-\widetilde{\mathbb{E}}[\varphi(Y)]\right| \leq\left|\mathbb{E}\left[\varphi\left(Y_{n}\right)\right]-\mathbb{E}\left[\bar{\varphi}\left(Y_{n}\right)\right]\right|+|\widetilde{\mathbb{E}}[\varphi(Y)]-\widetilde{\mathbb{E}}[\bar{\varphi}(Y)]| \\
& +\left|\mathbb{E}\left[\bar{\varphi}\left(Y_{n}\right)\right]-\widetilde{\mathbb{E}}[\bar{\varphi}(Y)]\right| \leq \varepsilon+\left|\mathbb{E}\left[\bar{\varphi}\left(Y_{n}\right)\right]-\widetilde{\mathbb{E}}[\bar{\varphi}(Y)]\right|
\end{aligned}
$$

Thus $\lim \sup _{n \rightarrow \infty}\left|\mathbb{E}\left[\varphi\left(Y_{n}\right)\right]-\widetilde{\mathbb{E}}[\varphi(Y)]\right| \leq \varepsilon$. The convergence must hold since $\varepsilon$ can be arbitrarily small.

Now let $\varphi$ be an arbitrary $C\left(\mathbb{R}^{d}\right)$-function with growth condition $|\varphi(x)| \leq$ $C\left(1+|x|^{p-1}\right)$. For each $N>0$ we can find $\varphi_{1}, \varphi_{2} \in C\left(\mathbb{R}^{d}\right)$ such that $\varphi=\varphi_{1}+\varphi_{2}$ where $\varphi_{1}$ has a compact support and $\varphi_{2}(x)=0$ for $|x| \leq N$, and $\left|\varphi_{2}(x)\right| \leq|\varphi(x)|$ for all $x$. It is clear that

$$
\left|\varphi_{2}(x)\right| \leq \frac{2 C\left(1+|x|^{p}\right)}{N} \text { for } x \in \mathbb{R}^{d} .
$$

Thus

$$
\begin{aligned}
\left|\mathbb{E}\left[\varphi\left(Y_{n}\right)\right]-\widetilde{\mathbb{E}}[\varphi(Y)]\right| & =\left|\mathbb{E}\left[\varphi_{1}\left(Y_{n}\right)+\varphi_{2}\left(Y_{n}\right)\right]-\widetilde{\mathbb{E}}\left[\varphi_{1}(Y)+\varphi_{2}(Y)\right]\right| \\
& \leq\left|\mathbb{E}\left[\varphi_{1}\left(Y_{n}\right)\right]-\widetilde{\mathbb{E}}\left[\varphi_{1}(Y)\right]\right|+\mathbb{E}\left[\left|\varphi_{2}\left(Y_{n}\right)\right|\right]+\widetilde{\mathbb{E}}\left[\left|\varphi_{2}(Y)\right|\right] \\
& \leq\left|\mathbb{E}\left[\varphi_{1}\left(Y_{n}\right)\right]-\widetilde{\mathbb{E}}\left[\varphi_{1}(Y)\right]\right|+\frac{2 C}{N}\left(2+\mathbb{E}\left[\left|Y_{n}\right|^{p}\right]+\widetilde{\mathbb{E}}\left[|Y|^{p}\right]\right) \\
& \leq\left|\mathbb{E}\left[\varphi_{1}\left(Y_{n}\right)\right]-\widetilde{\mathbb{E}}\left[\varphi_{1}(Y)\right]\right|+\frac{\bar{C}}{N},
\end{aligned}
$$

where $\bar{C}=2 C\left(2+\sup _{n} \mathbb{E}\left[\left|Y_{n}\right|^{p}\right]+\widetilde{\mathbb{E}}\left[|Y|^{p}\right]\right)$. We thus have $\lim _{\sup } \rightarrow \infty \mid \mathbb{E}\left[\varphi\left(Y_{n}\right)\right]-$ $\widetilde{\mathbb{E}}[\varphi(Y)] \mid \leq \frac{\bar{C}}{N}$. Since $N$ can be arbitrarily large, $\mathbb{E}\left[\varphi\left(Y_{n}\right)\right]$ must converge to $\widetilde{\mathbb{E}}[\varphi(Y)]$.

Exercise 3.10 Let $X_{i} \in \mathcal{H}, i=1,2, \cdots$, be such that $X_{i+1}$ is independent from $\left\{X_{1}, \cdots, X_{i}\right\}$, for each $i=1,2, \cdots$. We further assume that

$$
\begin{gathered}
\mathbb{E}\left[X_{i}\right]=-\mathbb{E}\left[-X_{i}\right]=0, \\
\lim _{i \rightarrow \infty} \mathbb{E}\left[X_{i}^{2}\right]=\bar{\sigma}^{2}<\infty, \lim _{i \rightarrow \infty}-\mathbb{E}\left[-X_{i}^{2}\right]=\underline{\sigma}^{2}, \\
\mathbb{E}\left[\left|X_{i}\right|^{2+\delta}\right] \leq M \text { for some } \delta>0 \text { and a constant } M .
\end{gathered}
$$

Prove that the sequence $\left\{\bar{S}_{n}\right\}_{n=1}^{\infty}$ defined by

$$
\bar{S}_{n}=\frac{1}{\sqrt{n}} \sum_{i=1}^{n} X_{i}
$$

converges in law to $X$, i.e.,

$$
\lim _{n \rightarrow \infty} \mathbb{E}\left[\varphi\left(\bar{S}_{n}\right)\right]=\mathbb{E}[\varphi(X)] \text { for } \varphi \in C_{b, l i p}(\mathbb{R}),
$$

where $X \sim N\left(\{0\} \times\left[\underline{\sigma}^{2}, \bar{\sigma}^{2}\right]\right)$.

In particular, if $\bar{\sigma}^{2}=\underline{\sigma}^{2}$, it becomes a classical central limit theorem. 


\section{Notes and Comments}

The contents of this chapter are mainly from Peng (2008) 105] (see also Peng (2007) 101]).

The notion of $G$-normal distribution was firstly introduced by Peng (2006) [100] for 1-dimensional case, and Peng (2008) 104 for multi-dimensional case. In the classical situation, a distribution satisfying equation (1.1) is said to be stable (see Lévy (1925) 77] and (1965) [78]). In this sense, our $G$-normal distribution can be considered as the most typical stable distribution under the framework of sublinear expectations.

Marinacci (1999) [83] used different notions of distributions and independence via capacity and the corresponding Choquet expectation to obtain a law of large numbers and a central limit theorem for non-additive probabilities (see also Maccheroni and Marinacci (2005) [84] ). But since a sublinear expectation can not be characterized by the corresponding capacity, our results can not be derived from theirs. In fact, our results show that the limit in CLT, under uncertainty, is a $G$-normal distribution in which the distribution uncertainty is not just the parameter of the classical normal distributions (see Exercise 2.2).

The notion of viscosity solutions plays a basic role in the definition and properties of $G$-normal distribution and maximal distribution. This notion was initially introduced by Crandall and Lions (1983) 29]. This is a fundamentally important notion in the theory of nonlinear parabolic and elliptic PDEs. Readers are referred to Crandall, Ishii and Lions (1992) [30] for rich references of the beautiful and powerful theory of viscosity solutions. For books on the theory of viscosity solutions and the related HJB equations, see Barles (1994) [8], Fleming and Soner (1992) [49] as well as Yong and Zhou (1999) [124].

We note that, for the case when the uniform elliptic condition holds, the viscosity solution (1.10) becomes a classical $C^{1+\frac{\alpha}{2}, 2+\alpha}$-solution (see Krylov (1987) 76] and the recent works in Cabre and Caffarelli (1997) [17] and Wang (1992) [119]). In 1-dimensional situation, when $\underline{\sigma}^{2}>0$, the $G$-equation becomes the following Barenblatt equation:

$$
\partial_{t} u+\gamma\left|\partial_{t} u\right|=\triangle u,|\gamma|<1 .
$$

This equation was first introduced by Barenblatt (1979) 7] (see also Avellaneda, Levy and Paras (1995) [5]). 


\section{Chapter III}

\section{$G$-Brownian Motion and Itô's Integral}

The aim of this chapter is to introduce the concept of $G$-Brownian motion, to study its properties and to construct Itô's integral with respect to $G$-Brownian motion. We emphasize here that our definition of $G$-Brownian motion is consistent with the classical one in the sense that if there is no volatility uncertainty. Our $G$-Brownian motion also has independent increments with identical $G$-normal distributions. $G$-Brownian motion has a very rich and interesting new structure which non-trivially generalizes the classical one. We thus can establish the related stochastic calculus, especially Itô's integrals and the related quadratic variation process. A very interesting new phenomenon of our $G$-Brownian motion is that its quadratic process also has independent increments which are identically distributed. The corresponding $G$-Itô's formula is obtained.

\section{$\S 1 \quad G$-Brownian Motion and its Characterization}

Definition 1.1 Let $(\Omega, \mathcal{H}, \mathbb{E})$ be a sublinear expectation space. $\left(X_{t}\right)_{t \geq 0}$ is called a d-dimensional stochastic process if for each $t \geq 0, X_{t}$ is a d-dimensional random vector in $\mathcal{H}$.

Let $G(\cdot): \mathbb{S}(d) \rightarrow \mathbb{R}$ be a given monotonic and sublinear function. By Theorem 2.1 in Chapter [1. there exists a bounded, convex and closed subset $\Sigma \subset \mathbb{S}_{+}(d)$ such that

$$
G(A)=\frac{1}{2} \sup _{B \in \Sigma}(A, B), \quad A \in \mathbb{S}(d) .
$$

By Section 2 in Chapter [1], we know that the $G$-normal distribution $N(\{0\} \times \Sigma)$ exists.

We now give the definition of $G$-Brownian motion. 
Definition $1.2 A$ d-dimensional process $\left(B_{t}\right)_{t \geq 0}$ on a sublinear expectation space $(\Omega, \mathcal{H}, \mathbb{E})$ is called a $G-B$ rownian motion if the following properties are satisfied:

(i) $B_{0}(\omega)=0$;

(ii) For each $t, s \geq 0$, the increment $B_{t+s}-B_{t}$ is $N(\{0\} \times s \Sigma)$-distributed and is independent from $\left(B_{t_{1}}, B_{t_{2}}, \cdots, B_{t_{n}}\right)$, for each $n \in \mathbb{N}$ and $0 \leq t_{1} \leq \cdots \leq t_{n} \leq t$.

Remark 1.3 We can prove that, for each $t_{0}>0,\left(B_{t+t_{0}}-B_{t_{0}}\right)_{t \geq 0}$ is a $G$ Brownian motion. For each $\lambda>0,\left(\lambda^{-\frac{1}{2}} B_{\lambda t}\right)_{t \geq 0}$ is also a G-Brownian motion. This is the scaling property of $G$-Brownian motion, which is the same as that of the classical Brownian motion.

We will denote in the rest of this book

$$
B_{t}^{\mathbf{a}}=\left\langle\mathbf{a}, B_{t}\right\rangle \quad \text { for each } \mathbf{a}=\left(a_{1}, \cdots, a_{d}\right)^{T} \in \mathbb{R}^{d} .
$$

By the above definition we have the following proposition which is important in stochastic calculus.

Proposition 1.4 Let $\left(B_{t}\right)_{t \geq 0}$ be a d-dimensional G-Brownian motion on a sublinear expectation space $(\Omega, \mathcal{H}, \mathbb{E})$. Then $\left(B_{t}^{\mathbf{a}}\right)_{t \geq 0}$ is a 1-dimensional $G_{\mathbf{a}^{-}}$ Brownian motion for each $\mathbf{a} \in \mathbb{R}^{d}$, where $G_{\mathbf{a}}(\alpha)=\frac{1}{2}\left(\sigma_{\mathbf{a a}^{T}}^{2} \alpha^{+}-\sigma_{-\mathbf{a a}^{T}}^{2} \alpha^{-}\right)$, $\sigma_{\mathbf{a a}^{T}}^{2}=2 G\left(\mathbf{a a}^{T}\right)=\mathbb{E}\left[\left\langle\mathbf{a}, B_{1}\right\rangle^{2}\right], \sigma_{-\mathbf{a a}^{T}}^{2}=-2 G\left(-\mathbf{a a}^{T}\right)=-\mathbb{E}\left[-\left\langle\mathbf{a}, B_{1}\right\rangle^{2}\right]$.

In particular, for each $t, s \geq 0, B_{t+s}^{\mathbf{a}}-B_{t}^{\mathbf{a}} \stackrel{d}{=} N\left(\{0\} \times\left[s \sigma_{-\mathbf{a a}^{T}}^{2}, s \sigma_{\mathbf{a a}^{T}}^{2}\right]\right)$.

Proposition 1.5 For each convex function $\varphi$, we have

$$
\mathbb{E}\left[\varphi\left(B_{t+s}^{\mathbf{a}}-B_{t}^{\mathbf{a}}\right)\right]=\frac{1}{\sqrt{2 \pi s \sigma_{\mathbf{a a}^{T}}^{2}}} \int_{-\infty}^{\infty} \varphi(x) \exp \left(-\frac{x^{2}}{2 s \sigma_{\mathbf{a a}^{T}}^{2}}\right) d x .
$$

For each concave function $\varphi$ and $\sigma_{-\mathbf{a a}^{T}}^{2}>0$, we have

$$
\mathbb{E}\left[\varphi\left(B_{t+s}^{\mathbf{a}}-B_{t}^{\mathbf{a}}\right)\right]=\frac{1}{\sqrt{2 \pi s \sigma_{-\mathbf{a a}^{T}}^{2}}} \int_{-\infty}^{\infty} \varphi(x) \exp \left(-\frac{x^{2}}{2 s \sigma_{-\mathbf{a a}^{T}}^{2}}\right) d x .
$$

In particular, we have

$$
\begin{aligned}
\mathbb{E}\left[\left(B_{t}^{\mathbf{a}}-B_{s}^{\mathbf{a}}\right)^{2}\right] & =\sigma_{\mathbf{a a}^{T}}^{2}(t-s), \quad \mathbb{E}\left[\left(B_{t}^{\mathbf{a}}-B_{s}^{\mathbf{a}}\right)^{4}\right]=3 \sigma_{\mathbf{a} \mathbf{a}^{T}}^{4}(t-s)^{2}, \\
\mathbb{E}\left[-\left(B_{t}^{\mathbf{a}}-B_{s}^{\mathbf{a}}\right)^{2}\right] & =-\sigma_{-\mathbf{a a}^{T}}^{2}(t-s), \quad \mathbb{E}\left[-\left(B_{t}^{\mathbf{a}}-B_{s}^{\mathbf{a}}\right)^{4}\right]=-3 \sigma_{-\mathbf{a a}^{T}}^{4}(t-s)^{2} .
\end{aligned}
$$

The following theorem gives a characterization of $G$-Brownian motion.

Theorem 1.6 Let $\left(B_{t}\right)_{t \geq 0}$ be a d-dimensional process defined on a sublinear expectation space $(\Omega, \mathcal{H}, \mathbb{E})$ such that

(i) $B_{0}(\omega)=0$;

(ii) For each $t, s \geq 0, B_{t+s}-B_{t}$ and $B_{s}$ are identically distributed and $B_{t+s}-B_{t}$ is independent from $\left(B_{t_{1}}, B_{t_{2}}, \cdots, B_{t_{n}}\right)$, for each $n \in \mathbb{N}$ and $0 \leq t_{1} \leq \cdots \leq t_{n} \leq$ $t$.

(iii) $\mathbb{E}\left[B_{t}\right]=\mathbb{E}\left[-B_{t}\right]=0$ and $\lim _{t \downarrow 0} \mathbb{E}\left[\left|B_{t}\right|^{3}\right] t^{-1}=0$.

Then $\left(B_{t}\right)_{t \geq 0}$ is a $G$-Brownian motion with $G(A)=\frac{1}{2} \mathbb{E}\left[\left\langle A B_{1}, B_{1}\right\rangle\right], A \in \mathbb{S}(d)$. 
Proof. We only need to prove that $B_{1}$ is $G$-normal distributed and $B_{t} \stackrel{d}{=} \sqrt{t} B_{1}$. We first prove that

$$
\mathbb{E}\left[\left\langle A B_{t}, B_{t}\right\rangle\right]=2 G(A) t, \quad A \in \mathbb{S}(d) .
$$

For each given $A \in \mathbb{S}(d)$, we set $b(t)=\mathbb{E}\left[\left\langle A B_{t}, B_{t}\right\rangle\right]$. Then $b(0)=0$ and $|b(t)| \leq$ $|A|\left(\mathbb{E}\left[\left|B_{t}\right|^{3}\right]\right)^{2 / 3} \rightarrow 0$ as $t \rightarrow 0$. Since for each $t, s \geq 0$,

$$
\begin{aligned}
b(t+s) & =\mathbb{E}\left[\left\langle A B_{t+s}, B_{t+s}\right\rangle\right]=\hat{\mathbb{E}}\left[\left\langle A\left(B_{t+s}-B_{s}+B_{s}\right), B_{t+s}-B_{s}+B_{s}\right\rangle\right] \\
& =\mathbb{E}\left[\left\langle A\left(B_{t+s}-B_{s}\right),\left(B_{t+s}-B_{s}\right)\right\rangle+\left\langle A B_{s}, B_{s}\right\rangle+2\left\langle A\left(B_{t+s}-B_{s}\right), B_{s}\right\rangle\right] \\
& =b(t)+b(s),
\end{aligned}
$$

we have $b(t)=b(1) t=2 G(A) t$.

We now prove that $B_{1}$ is $G$-normal distributed and $B_{t} \stackrel{d}{=} \sqrt{t} B_{1}$. For this, we just need to prove that, for each fixed $\varphi \in C_{b . L i p}\left(\mathbb{R}^{d}\right)$, the function

$$
u(t, x):=\mathbb{E}\left[\varphi\left(x+B_{t}\right)\right], \quad(t, x) \in[0, \infty) \times \mathbb{R}^{d}
$$

is the viscosity solution of the following $G$-heat equation:

$$
\partial_{t} u-G\left(D^{2} u\right)=0,\left.\quad u\right|_{t=0}=\varphi .
$$

We first prove that $u$ is Lipschitz in $x$ and $\frac{1}{2}$-Hölder continuous in $t$. In fact, for each fixed $t, u(t, \cdot) \in C_{b . L i p}\left(\mathbb{R}^{d}\right)$ since

$$
\begin{aligned}
|u(t, x)-u(t, y)| & =\left|\mathbb{E}\left[\varphi\left(x+B_{t}\right)\right]-\mathbb{E}\left[\varphi\left(y+B_{t}\right)\right]\right| \\
& \leq \mathbb{E}\left[\left|\varphi\left(x+B_{t}\right)-\varphi\left(y+B_{t}\right)\right|\right] \\
& \leq C|x-y|,
\end{aligned}
$$

where $C$ is Lipschitz constant of $\varphi$.

For each $\delta \in[0, t]$, since $B_{t}-B_{\delta}$ is independent from $B_{\delta}$, we also have

$$
\begin{aligned}
u(t, x) & =\mathbb{E}\left[\varphi\left(x+B_{\delta}+\left(B_{t}-B_{\delta}\right)\right]\right. \\
& =\mathbb{E}\left[\mathbb{E}\left[\varphi\left(y+\left(B_{t}-B_{\delta}\right)\right)\right]_{y=x+B_{\delta}}\right],
\end{aligned}
$$

hence

$$
u(t, x)=\mathbb{E}\left[u\left(t-\delta, x+B_{\delta}\right)\right]
$$

Thus

$$
\begin{aligned}
|u(t, x)-u(t-\delta, x)| & =\left|\mathbb{E}\left[u\left(t-\delta, x+B_{\delta}\right)-u(t-\delta, x)\right]\right| \\
& \leq \mathbb{E}\left[\left|u\left(t-\delta, x+B_{\delta}\right)-u(t-\delta, x)\right|\right] \\
& \leq \mathbb{E}\left[C\left|B_{\delta}\right|\right] \leq C \sqrt{2 G(I)} \sqrt{\delta} .
\end{aligned}
$$

To prove that $u$ is a viscosity solution of (1.1), we fix $(t, x) \in(0, \infty) \times \mathbb{R}^{d}$ and let $v \in C_{b}^{2,3}\left([0, \infty) \times \mathbb{R}^{d}\right)$ be such that $v \geq u$ and $v(t, x)=u(t, x)$. From (1.2) we have

$$
v(t, x)=\mathbb{E}\left[u\left(t-\delta, x+B_{\delta}\right)\right] \leq \mathbb{E}\left[v\left(t-\delta, x+B_{\delta}\right)\right] .
$$


Therefore by Taylor's expansion,

$$
\begin{aligned}
0 & \leq \mathbb{E}\left[v\left(t-\delta, x+B_{\delta}\right)-v(t, x)\right] \\
& =\mathbb{E}\left[v\left(t-\delta, x+B_{\delta}\right)-v\left(t, x+B_{\delta}\right)+\left(v\left(t, x+B_{\delta}\right)-v(t, x)\right)\right] \\
& =\mathbb{E}\left[-\partial_{t} v(t, x) \delta+\left\langle D v(t, x), B_{\delta}\right\rangle+\frac{1}{2}\left\langle D^{2} v(t, x) B_{\delta}, B_{\delta}\right\rangle+I_{\delta}\right] \\
& \leq-\partial_{t} v(t, x) \delta+\frac{1}{2} \mathbb{E}\left[\left\langle D^{2} v(t, x) B_{\delta}, B_{\delta}\right\rangle\right]+\mathbb{E}\left[I_{\delta}\right] \\
& =-\partial_{t} v(t, x) \delta+G\left(D^{2} v(t, x)\right) \delta+\mathbb{E}\left[I_{\delta}\right]
\end{aligned}
$$

where

$$
\begin{aligned}
I_{\delta} & =\int_{0}^{1}-\left[\partial_{t} v\left(t-\beta \delta, x+B_{\delta}\right)-\partial_{t} v(t, x)\right] \delta d \beta \\
& +\int_{0}^{1} \int_{0}^{1}\left\langle\left(D^{2} v\left(t, x+\alpha \beta B_{\delta}\right)-D^{2} v(t, x)\right) B_{\delta}, B_{\delta}\right\rangle \alpha d \beta d \alpha .
\end{aligned}
$$

With the assumption (iii) we can check that $\lim _{\delta \downarrow 0} \mathbb{E}\left[\left|I_{\delta}\right|\right] \delta^{-1}=0$, from which we get $\partial_{t} v(t, x)-G\left(D^{2} v(t, x)\right) \leq 0$, hence $u$ is a viscosity subsolution of (1.1). We can analogously prove that $u$ is a viscosity supersolution. Thus $u$ is a viscosity solution and $\left(B_{t}\right)_{t \geq 0}$ is a $G$-Brownian motion. The proof is complete.

Exercise 1.7 Let $B_{t}$ be a 1-dimensional Brownian motion, and $B_{1} \stackrel{d}{=} N(\{0\} \times$ $\left.\left[\underline{\sigma}^{2}, \bar{\sigma}^{2}\right]\right)$. Prove that for each $m \in \mathbb{N}$,

$$
\hat{\mathbb{E}}\left[\left|B_{t}\right|^{m}\right]= \begin{cases}2(m-1) ! ! \bar{\sigma}^{m} t^{\frac{m}{2}} / \sqrt{2 \pi} & m \text { is odd } \\ (m-1) ! ! \bar{\sigma}^{m} t^{\frac{m}{2}} & m \text { is even } .\end{cases}
$$

\section{$\S 2$ Existence of $G$-Brownian Motion}

In the rest of this book, we denote by $\Omega=C_{0}^{d}\left(\mathbb{R}^{+}\right)$the space of all $\mathbb{R}^{d}$-valued continuous paths $\left(\omega_{t}\right)_{t \in \mathbb{R}^{+}}$, with $\omega_{0}=0$, equipped with the distance

$$
\rho\left(\omega^{1}, \omega^{2}\right):=\sum_{i=1}^{\infty} 2^{-i}\left[\left(\max _{t \in[0, i]}\left|\omega_{t}^{1}-\omega_{t}^{2}\right|\right) \wedge 1\right] .
$$

For each fixed $T \in[0, \infty)$, we set $\Omega_{T}:=\{\omega \cdot \wedge T: \omega \in \Omega\}$. We will consider the canonical process $B_{t}(\omega)=\omega_{t}, t \in[0, \infty)$, for $\omega \in \Omega$.

For each fixed $T \in[0, \infty)$, we set

$L_{i p}\left(\Omega_{T}\right):=\left\{\varphi\left(B_{t_{1} \wedge T}, \cdots, B_{t_{n} \wedge T}\right): n \in \mathbb{N}, t_{1}, \cdots, t_{n} \in[0, \infty), \varphi \in C_{l . L i p}\left(\mathbb{R}^{d \times n}\right)\right\}$.

It is clear that $L_{i p}\left(\Omega_{t}\right) \subseteq L_{i p}\left(\Omega_{T}\right)$, for $t \leq T$. We also set

$$
L_{i p}(\Omega):=\bigcup_{n=1}^{\infty} L_{i p}\left(\Omega_{n}\right) \text {. }
$$


Remark 2.1 It is clear that $C_{l . L i p}\left(\mathbb{R}^{d \times n}\right), L_{i p}\left(\Omega_{T}\right)$ and $L_{i p}(\Omega)$ are vector lattices. Moreover, note that $\varphi, \psi \in C_{\text {l.Lip }}\left(\mathbb{R}^{d \times n}\right)$ imply $\varphi \cdot \psi \in C_{\text {l.Lip }}\left(\mathbb{R}^{d \times n}\right)$, then $X, Y \in L_{i p}\left(\Omega_{T}\right)$ imply $X \cdot Y \in L_{i p}\left(\Omega_{T}\right)$. In particular, for each $t \in[0, \infty)$, $B_{t} \in L_{i p}(\Omega)$.

Let $G(\cdot): \mathbb{S}(d) \rightarrow \mathbb{R}$ be a given monotonic and sublinear function. In the following, we want to construct a sublinear expectation on $\left(\Omega, L_{i p}(\Omega)\right)$ such that the canonical process $\left(B_{t}\right)_{t \geq 0}$ is a $G$-Brownian motion. For this, we first construct a sequence of $d$-dimensional random vectors $\left(\xi_{i}\right)_{i=1}^{\infty}$ on a sublinear expectation space $(\widetilde{\Omega}, \widetilde{\mathcal{H}}, \widetilde{\mathbb{E}})$ such that $\xi_{i}$ is $G$-normal distributed and $\xi_{i+1}$ is independent from $\left(\xi_{1}, \cdots, \xi_{i}\right)$ for each $i=1,2, \cdots$.

We now introduce a sublinear expectation $\hat{\mathbb{E}}$ defined on $L_{i p}(\Omega)$ via the following procedure: for each $X \in L_{i p}(\Omega)$ with

$$
X=\varphi\left(B_{t_{1}}-B_{t_{0}}, B_{t_{2}}-B_{t_{1}}, \cdots, B_{t_{n}}-B_{t_{n-1}}\right)
$$

for some $\varphi \in C_{l . L i p}\left(\mathbb{R}^{d \times n}\right)$ and $0=t_{0}<t_{1}<\cdots<t_{n}<\infty$, we set

$$
\begin{gathered}
\hat{\mathbb{E}}\left[\varphi\left(B_{t_{1}}-B_{t_{0}}, B_{t_{2}}-B_{t_{1}}, \cdots, B_{t_{n}}-B_{t_{n-1}}\right)\right] \\
:=\widetilde{\mathbb{E}}\left[\varphi\left(\sqrt{t_{1}-t_{0}} \xi_{1}, \cdots, \sqrt{t_{n}-t_{n-1}} \xi_{n}\right)\right] .
\end{gathered}
$$

The related conditional expectation of $X=\varphi\left(B_{t_{1}}, B_{t_{2}}-B_{t_{1}}, \cdots, B_{t_{n}}-\right.$ $B_{t_{n-1}}$ ) under $\Omega_{t_{j}}$ is defined by

$$
\begin{aligned}
\hat{\mathbb{E}}\left[X \mid \Omega_{t_{j}}\right] & =\hat{\mathbb{E}}\left[\varphi\left(B_{t_{1}}, B_{t_{2}}-B_{t_{1}}, \cdots, B_{t_{n}}-B_{t_{n-1}}\right) \mid \Omega_{t_{j}}\right] \\
& :=\psi\left(B_{t_{1}}, \cdots, B_{t_{j}}-B_{t_{j-1}}\right),
\end{aligned}
$$

where

$$
\psi\left(x_{1}, \cdots, x_{j}\right)=\widetilde{\mathbb{E}}\left[\varphi\left(x_{1}, \cdots, x_{j}, \sqrt{t_{j+1}-t_{j}} \xi_{j+1}, \cdots, \sqrt{t_{n}-t_{n-1}} \xi_{n}\right)\right] .
$$

It is easy to check that $\hat{\mathbb{E}}[\cdot]$ consistently defines a sublinear expectation on $L_{i p}(\Omega)$ and $\left(B_{t}\right)_{t \geq 0}$ is a $G$-Brownian motion. Since $L_{i p}\left(\Omega_{T}\right) \subseteq L_{i p}(\Omega), \hat{\mathbb{E}}[\cdot]$ is also a sublinear expectation on $L_{i p}\left(\Omega_{T}\right)$.

Definition 2.2 The sublinear expectation $\hat{\mathbb{E}}[\cdot]: L_{i p}(\Omega) \rightarrow \mathbb{R}$ defined through the above procedure is called a $G$-expectation. The corresponding canonical process $\left(B_{t}\right)_{t \geq 0}$ on the sublinear expectation space $\left(\Omega, L_{i p}(\Omega), \widehat{\mathbb{E}}\right)$ is called a $G$-Brownian motion.

In the rest of this book, when we talk about $G$-Brownian motion, we mean that the canonical process $\left(B_{t}\right)_{t \geq 0}$ is under $G$-expectation.

Proposition 2.3 We list the properties of $\hat{\mathbb{E}}\left[\cdot \mid \Omega_{t}\right]$ that hold for each $X, Y \in L_{i p}(\Omega)$ :

(i) If $X \geq Y$, then $\hat{\mathbb{E}}\left[X \mid \Omega_{t}\right] \geq \hat{\mathbb{E}}\left[Y \mid \Omega_{t}\right]$. 
(ii) $\hat{\mathbb{E}}\left[\eta \mid \Omega_{t}\right]=\eta$, for each $t \in[0, \infty)$ and $\eta \in L_{i p}\left(\Omega_{t}\right)$.

(iii) $\hat{\mathbb{E}}\left[X \mid \Omega_{t}\right]-\hat{\mathbb{E}}\left[Y \mid \Omega_{t}\right] \leq \hat{\mathbb{E}}\left[X-Y \mid \Omega_{t}\right]$.

(iv) $\hat{\mathbb{E}}\left[\eta X \mid \Omega_{t}\right]=\eta^{+} \hat{\mathbb{E}}\left[X \mid \Omega_{t}\right]+\eta^{-} \hat{\mathbb{E}}\left[-X \mid \Omega_{t}\right]$ for each $\eta \in L_{i p}\left(\Omega_{t}\right)$.

(v) $\hat{\mathbb{E}}\left[\hat{\mathbb{E}}\left[X \mid \Omega_{t}\right] \mid \Omega_{s}\right]=\hat{\mathbb{E}}\left[X \mid \Omega_{t \wedge s}\right]$, in particular, $\hat{\mathbb{E}}\left[\hat{\mathbb{E}}\left[X \mid \Omega_{t}\right]\right]=\hat{\mathbb{E}}[X]$.

For each $X \in L_{i p}\left(\Omega^{t}\right), \hat{\mathbb{E}}\left[X \mid \Omega_{t}\right]=\hat{\mathbb{E}}[X]$, where $L_{i p}\left(\Omega^{t}\right)$ is the linear space of random variables with the form

$$
\begin{aligned}
& \varphi\left(B_{t_{2}}-B_{t_{1}}, B_{t_{3}}-B_{t_{2}}, \cdots, B_{t_{n+1}}-B_{t_{n}}\right), \\
& \quad n=1,2, \cdots, \varphi \in C_{l . L i p}\left(\mathbb{R}^{d \times n}\right), t_{1}, \cdots, t_{n}, t_{n+1} \in[t, \infty) .
\end{aligned}
$$

Remark 2.4 (ii) and (iii) imply

$$
\hat{\mathbb{E}}\left[X+\eta \mid \Omega_{t}\right]=\hat{\mathbb{E}}\left[X \mid \Omega_{t}\right]+\eta \text { for } \eta \in L_{i p}\left(\Omega_{t}\right) .
$$

We now consider the completion of sublinear expectation space $\left(\Omega, L_{i p}(\Omega), \hat{\mathbb{E}}\right)$. We denote by $L_{G}^{p}(\Omega), p \geq 1$, the completion of $L_{i p}(\Omega)$ under the norm $\|X\|_{p}:=\left(\hat{\mathbb{E}}\left[|X|^{p}\right]\right)^{1 / p}$. Similarly, we can define $L_{G}^{p}\left(\Omega_{T}\right), L_{G}^{p}\left(\Omega_{T}^{t}\right)$ and $L_{G}^{p}\left(\Omega^{t}\right)$. It is clear that for each $0 \leq t \leq T<\infty, L_{G}^{p}\left(\Omega_{t}\right) \subseteq L_{G}^{p}\left(\Omega_{T}\right) \subseteq L_{G}^{p}(\Omega)$.

According to Sec凹 in Chap, $\hat{\mathbb{E}}[\cdot]$ can be continuously extended to a sublinear expectation on $\left(\Omega, L_{G}^{1}(\Omega)\right)$ still denoted by $\hat{\mathbb{E}}[\cdot]$. We now consider the extension of conditional expectations. For each fixed $t \leq T$, the conditional $G$-expectation $\hat{\mathbb{E}}\left[\cdot \mid \Omega_{t}\right]: L_{i p}\left(\Omega_{T}\right) \rightarrow L_{i p}\left(\Omega_{t}\right)$ is a continuous mapping under $\|\cdot\|$. Indeed, we have

$$
\hat{\mathbb{E}}\left[X \mid \Omega_{t}\right]-\hat{\mathbb{E}}\left[Y \mid \Omega_{t}\right] \leq \hat{\mathbb{E}}\left[X-Y \mid \Omega_{t}\right] \leq \hat{\mathbb{E}}\left[|X-Y| \mid \Omega_{t}\right],
$$

then

$$
\left|\hat{\mathbb{E}}\left[X \mid \Omega_{t}\right]-\hat{\mathbb{E}}\left[Y \mid \Omega_{t}\right]\right| \leq \hat{\mathbb{E}}\left[|X-Y| \mid \Omega_{t}\right] .
$$

We thus obtain

$$
\left\|\hat{\mathbb{E}}\left[X \mid \Omega_{t}\right]-\hat{\mathbb{E}}\left[Y \mid \Omega_{t}\right]\right\| \leq\|X-Y\| .
$$

It follows that $\hat{\mathbb{E}}\left[\cdot \mid \Omega_{t}\right]$ can be also extended as a continuous mapping

$$
\hat{\mathbb{E}}\left[\cdot \mid \Omega_{t}\right]: L_{G}^{1}\left(\Omega_{T}\right) \rightarrow L_{G}^{1}\left(\Omega_{t}\right) .
$$

If the above $T$ is not fixed, then we can obtain $\hat{\mathbb{E}}\left[\cdot \mid \Omega_{t}\right]: L_{G}^{1}(\Omega) \rightarrow L_{G}^{1}\left(\Omega_{t}\right)$.

Remark 2.5 The above proposition also holds for $X, Y \in L_{G}^{1}(\Omega)$. But in (iv), $\eta \in L_{G}^{1}\left(\Omega_{t}\right)$ should be bounded, since $X, Y \in L_{G}^{1}(\Omega)$ does not imply $X \cdot Y \in$ $L_{G}^{1}(\Omega)$.

In particular, we have the following independence:

$$
\hat{\mathbb{E}}\left[X \mid \Omega_{t}\right]=\hat{\mathbb{E}}[X], \quad \forall X \in L_{G}^{1}\left(\Omega^{t}\right) .
$$

We give the following definition similar to the classical one: 
Definition 2.6 An n-dimensional random vector $Y \in\left(L_{G}^{1}(\Omega)\right)^{n}$ is said to be independent from $\Omega_{t}$ for some given $t$ if for each $\varphi \in C_{b . L i p}\left(\mathbb{R}^{n}\right)$ we have

$$
\hat{\mathbb{E}}\left[\varphi(Y) \mid \Omega_{t}\right]=\hat{\mathbb{E}}[\varphi(Y)]
$$

Remark 2.7 Just as in the classical situation, the increments of $G$-Brownian motion $\left(B_{t+s}-B_{t}\right)_{s \geq 0}$ are independent from $\Omega_{t}$.

The following property is very useful.

Proposition 2.8 Let $X, Y \in L_{G}^{1}(\Omega)$ be such that $\hat{\mathbb{E}}\left[Y \mid \Omega_{t}\right]=-\hat{\mathbb{E}}\left[-Y \mid \Omega_{t}\right]$, for some $t \in[0, T]$. Then we have

$$
\hat{\mathbb{E}}\left[X+Y \mid \Omega_{t}\right]=\hat{\mathbb{E}}\left[X \mid \Omega_{t}\right]+\hat{\mathbb{E}}\left[Y \mid \Omega_{t}\right] .
$$

In particular, if $\hat{\mathbb{E}}\left[Y \mid \Omega_{t}\right]=\hat{\mathbb{E}}_{G}\left[-Y \mid \Omega_{t}\right]=0$, then $\hat{\mathbb{E}}\left[X+Y \mid \Omega_{t}\right]=\hat{\mathbb{E}}\left[X \mid \Omega_{t}\right]$.

Proof. This follows from the following two inequalities:

$$
\begin{aligned}
& \hat{\mathbb{E}}\left[X+Y \mid \Omega_{t}\right] \leq \hat{\mathbb{E}}\left[X \mid \Omega_{t}\right]+\hat{\mathbb{E}}\left[Y \mid \Omega_{t}\right], \\
& \hat{\mathbb{E}}\left[X+Y \mid \Omega_{t}\right] \geq \hat{\mathbb{E}}\left[X \mid \Omega_{t}\right]-\hat{\mathbb{E}}\left[-Y \mid \Omega_{t}\right]=\hat{\mathbb{E}}\left[X \mid \Omega_{t}\right]+\hat{\mathbb{E}}\left[Y \mid \Omega_{t}\right] .
\end{aligned}
$$

Example 2.9 For each fixed $\mathbf{a} \in \mathbb{R}^{d}, s \leq t$, we have

$$
\begin{aligned}
\hat{\mathbb{E}}\left[B_{t}^{\mathbf{a}}-B_{s}^{\mathbf{a}} \mid \Omega_{s}\right] & =0, \quad \hat{\mathbb{E}}\left[-\left(B_{t}^{\mathbf{a}}-B_{s}^{\mathbf{a}}\right) \mid \Omega_{s}\right]=0, \\
\hat{\mathbb{E}}\left[\left(B_{t}^{\mathbf{a}}-B_{s}^{\mathbf{a}}\right)^{2} \mid \Omega_{s}\right] & =\sigma_{\mathbf{a a}^{T}}^{2}(t-s), \quad \hat{\mathbb{E}}\left[-\left(B_{t}^{\mathbf{a}}-B_{s}^{\mathbf{a}}\right)^{2} \mid \Omega_{s}\right]=-\sigma_{-\mathbf{a a}^{T}}^{2}(t-s), \\
\hat{\mathbb{E}}\left[\left(B_{t}^{\mathbf{a}}-B_{s}^{\mathbf{a}}\right)^{4} \mid \Omega_{s}\right] & =3 \sigma_{\mathbf{a a}^{T}}^{4}(t-s)^{2}, \quad \hat{\mathbb{E}}\left[-\left(B_{t}^{\mathbf{a}}-B_{s}^{\mathbf{a}}\right)^{4} \mid \Omega_{s}\right]=-3 \sigma_{-\mathbf{a a}^{T}}^{4}(t-s)^{2},
\end{aligned}
$$

where $\sigma_{\mathbf{a a}^{T}}^{2}=2 G\left(\mathbf{a a}^{T}\right)$ and $\sigma_{-\mathbf{a a}^{T}}^{2}=-2 G\left(-\mathbf{a a}^{T}\right)$.

Example 2.10 For each $\mathbf{a} \in \mathbb{R}^{d}, n \in \mathbb{N}, 0 \leq t \leq T, X \in L_{G}^{1}\left(\Omega_{t}\right)$ and $\varphi \in$ $C_{\text {l.Lip }}(\mathbb{R})$, we have

$$
\begin{aligned}
\hat{\mathbb{E}}\left[X \varphi\left(B_{T}^{\mathbf{a}}-B_{t}^{\mathbf{a}}\right) \mid \Omega_{t}\right] & =X^{+} \hat{\mathbb{E}}\left[\varphi\left(B_{T}^{\mathbf{a}}-B_{t}^{\mathbf{a}}\right) \mid \Omega_{t}\right]+X^{-} \hat{\mathbb{E}}\left[-\varphi\left(B_{T}^{\mathbf{a}}-B_{t}^{\mathbf{a}}\right) \mid \Omega_{t}\right] \\
& =X^{+} \hat{\mathbb{E}}\left[\varphi\left(B_{T}^{\mathbf{a}}-B_{t}^{\mathbf{a}}\right)\right]+X^{-} \hat{\mathbb{E}}\left[-\varphi\left(B_{T}^{\mathbf{a}}-B_{t}^{\mathbf{a}}\right)\right] .
\end{aligned}
$$

In particular, we have

$$
\hat{\mathbb{E}}\left[X\left(B_{T}^{\mathbf{a}}-B_{t}^{\mathbf{a}}\right) \mid \Omega_{t}\right]=X^{+} \hat{\mathbb{E}}\left[\left(B_{T}^{\mathbf{a}}-B_{t}^{\mathbf{a}}\right)\right]+X^{-} \hat{\mathbb{E}}\left[-\left(B_{T}^{\mathbf{a}}-B_{t}^{\mathbf{a}}\right)\right]=0 .
$$

This, together with Proposition 2.8, yields

$$
\hat{\mathbb{E}}\left[Y+X\left(B_{T}^{\mathbf{a}}-B_{t}^{\mathbf{a}}\right) \mid \Omega_{t}\right]=\hat{\mathbb{E}}\left[Y \mid \Omega_{t}\right], \quad Y \in L_{G}^{1}(\Omega) .
$$


We also have

$$
\begin{aligned}
\hat{\mathbb{E}}\left[X\left(B_{T}^{\mathbf{a}}-B_{t}^{\mathbf{a}}\right)^{2} \mid \Omega_{t}\right] & =X^{+} \hat{\mathbb{E}}\left[\left(B_{T}^{\mathbf{a}}-B_{t}^{\mathbf{a}}\right)^{2}\right]+X^{-} \hat{\mathbb{E}}\left[-\left(B_{T}^{\mathbf{a}}-B_{t}^{\mathbf{a}}\right)^{2}\right] \\
& =\left[X^{+} \sigma_{\mathbf{a a}^{T}}^{2}-X^{-} \sigma_{-\mathbf{a a}^{T}}^{2}\right](T-t)
\end{aligned}
$$

and

$$
\begin{aligned}
\hat{\mathbb{E}}\left[X\left(B_{T}^{\mathbf{a}}-B_{t}^{\mathbf{a}}\right)^{2 n-1} \mid \Omega_{t}\right] & =X^{+} \hat{\mathbb{E}}\left[\left(B_{T}^{\mathbf{a}}-B_{t}^{\mathbf{a}}\right)^{2 n-1}\right]+X^{-} \hat{\mathbb{E}}\left[-\left(B_{T}^{\mathbf{a}}-B_{t}^{\mathbf{a}}\right)^{2 n-1}\right] \\
& =|X| \hat{\mathbb{E}}\left[\left(B_{T-t}^{\mathbf{a}}\right)^{2 n-1}\right] .
\end{aligned}
$$

Example 2.11 Since

$$
\hat{\mathbb{E}}\left[2 B_{s}^{\mathbf{a}}\left(B_{t}^{\mathbf{a}}-B_{s}^{\mathbf{a}}\right) \mid \Omega_{s}\right]=\hat{\mathbb{E}}\left[-2 B_{s}^{\mathbf{a}}\left(B_{t}^{\mathbf{a}}-B_{s}^{\mathbf{a}}\right) \mid \Omega_{s}\right]=0,
$$

we have

$$
\begin{aligned}
\hat{\mathbb{E}}\left[\left(B_{t}^{\mathbf{a}}\right)^{2}-\left(B_{s}^{\mathbf{a}}\right)^{2} \mid \Omega_{s}\right] & =\hat{\mathbb{E}}\left[\left(B_{t}^{\mathbf{a}}-B_{s}^{\mathbf{a}}+B_{s}^{\mathbf{a}}\right)^{2}-\left(B_{s}^{\mathbf{a}}\right)^{2} \mid \Omega_{s}\right] \\
& =\hat{\mathbb{E}}\left[\left(B_{t}^{\mathbf{a}}-B_{s}^{\mathbf{a}}\right)^{2}+2\left(B_{t}^{\mathbf{a}}-B_{s}^{\mathbf{a}}\right) B_{s}^{\mathbf{a}} \mid \Omega_{s}\right] \\
& =\sigma_{\mathbf{a a}^{T}}^{2}(t-s) .
\end{aligned}
$$

Exercise 2.12 Show that if $X \in \operatorname{Lip}\left(\Omega_{T}\right)$ and $\hat{\mathbb{E}}[X]=-\hat{\mathbb{E}}[-X]$, then $\hat{\mathbb{E}}[X]=$ $E_{P}[X]$, where $P$ is a Wiener measure on $\Omega$.

Exercise 2.13 For each $s, t \geq 0$, we set $B_{t}^{s}:=B_{t+s}-B_{s}$. Let $\eta=\left(\eta_{i j}\right)_{i, j=1}^{d} \in$ $L_{G}^{1}\left(\Omega_{s} ; \mathbb{S}(d)\right)$. Prove that

$$
\hat{\mathbb{E}}\left[\left\langle\eta B_{t}^{s}, B_{t}^{s}\right\rangle \mid \Omega_{s}\right]=2 G(\eta) t
$$

\section{$\S 3$ Itô's Integral with $G$-Brownian Motion}

Definition 3.1 For $T \in \mathbb{R}^{+}$, a partition $\pi_{T}$ of $[0, T]$ is a finite ordered subset $\pi_{T}=\left\{t_{0}, t_{1}, \cdots, t_{N}\right\}$ such that $0=t_{0}<t_{1}<\cdots<t_{N}=T$.

$$
\mu\left(\pi_{T}\right):=\max \left\{\left|t_{i+1}-t_{i}\right|: i=0,1, \cdots, N-1\right\} .
$$

We use $\pi_{T}^{N}=\left\{t_{0}^{N}, t_{1}^{N}, \cdots, t_{N}^{N}\right\}$ to denote a sequence of partitions of $[0, T]$ such that $\lim _{N \rightarrow \infty} \mu\left(\pi_{T}^{N}\right)=0$.

Let $p \geq 1$ be fixed. We consider the following type of simple processes: for a given partition $\pi_{T}=\left\{t_{0}, \cdots, t_{N}\right\}$ of $[0, T]$ we set

$$
\eta_{t}(\omega)=\sum_{k=0}^{N-1} \xi_{k}(\omega) \mathbf{I}_{\left[t_{k}, t_{k+1}\right)}(t),
$$

where $\xi_{k} \in L_{G}^{p}\left(\Omega_{t_{k}}\right), k=0,1,2, \cdots, N-1$ are given. The collection of these processes is denoted by $M_{G}^{p, 0}(0, T)$. 
Definition 3.2 For an $\eta \in M_{G}^{p, 0}(0, T)$ with $\eta_{t}(\omega)=\sum_{k=0}^{N-1} \xi_{k}(\omega) \mathbf{I}_{\left[t_{k}, t_{k+1}\right)}(t)$, the related Bochner integral is

$$
\int_{0}^{T} \eta_{t}(\omega) d t:=\sum_{k=0}^{N-1} \xi_{k}(\omega)\left(t_{k+1}-t_{k}\right) .
$$

For each $\eta \in M_{G}^{p, 0}(0, T)$, we set

$$
\widetilde{\mathbb{E}}_{T}[\eta]:=\frac{1}{T} \hat{\mathbb{E}}\left[\int_{0}^{T} \eta_{t} d t\right]=\frac{1}{T} \hat{\mathbb{E}}\left[\sum_{k=0}^{N-1} \xi_{k}(\omega)\left(t_{k+1}-t_{k}\right)\right] .
$$

It is easy to check that $\widetilde{\mathbb{E}}_{T}: M_{G}^{p, 0}(0, T) \rightarrow \mathbb{R}$ forms a sublinear expectation. We then can introduce a natural norm $\|\eta\|_{M_{G}^{p}(0, T)}$, under which, $M_{G}^{p, 0}(0, T)$ can be extended to $M_{G}^{p}(0, T)$ which is a Banach space.

Definition 3.3 For each $p \geq 1$, we denote by $M_{G}^{p}(0, T)$ the completion of $M_{G}{ }^{p, 0}(0, T)$ under the norm

$$
\|\eta\|_{M_{G}^{p}(0, T)}:=\left\{\hat{\mathbb{E}}\left[\int_{0}^{T}\left|\eta_{t}\right|^{p} d t\right]\right\}^{1 / p} .
$$

It is clear that $M_{G}^{p}(0, T) \supset M_{G}^{q}(0, T)$ for $1 \leq p \leq q$. We also use $M_{G}^{p}\left(0, T ; \mathbb{R}^{n}\right)$ for all $n$-dimensional stochastic processes $\eta_{t}=\left(\eta_{t}^{1}, \cdots, \eta_{t}^{n}\right), t \geq 0$ with $\eta_{t}^{i} \in$ $M_{G}^{p}(0, T), i=1,2, \cdots, n$.

We now give the definition of Itô's integral. For simplicity, we first introduce Itô's integral with respect to 1-dimensional $G$-Brownian motion.

Let $\left(B_{t}\right)_{t \geq 0}$ be a 1-dimensional $G$-Brownian motion with $G(\alpha)=\frac{1}{2}\left(\bar{\sigma}^{2} \alpha^{+}-\right.$ $\left.\underline{\sigma}^{2} \alpha^{-}\right)$, where $0 \leq \underline{\sigma} \leq \bar{\sigma}<\infty$.

Definition 3.4 For each $\eta \in M_{G}^{2,0}(0, T)$ of the form

$$
\eta_{t}(\omega)=\sum_{j=0}^{N-1} \xi_{j}(\omega) \mathbf{I}_{\left[t_{j}, t_{j+1}\right)}(t)
$$

we define

$$
I(\eta)=\int_{0}^{T} \eta_{t} d B_{t}:=\sum_{j=0}^{N-1} \xi_{j}\left(B_{t_{j+1}}-B_{t_{j}}\right) .
$$

Lemma 3.5 The mapping $I: M_{G}^{2,0}(0, T) \rightarrow L_{G}^{2}\left(\Omega_{T}\right)$ is a continuous linear mapping and thus can be continuously extended to $I: M_{G}^{2}(0, T) \rightarrow L_{G}^{2}\left(\Omega_{T}\right)$. We have

$$
\begin{aligned}
\hat{\mathbb{E}}\left[\int_{0}^{T} \eta_{t} d B_{t}\right] & =0 \\
\hat{\mathbb{E}}\left[\left(\int_{0}^{T} \eta_{t} d B_{t}\right)^{2}\right] & \leq \bar{\sigma}^{2} \hat{\mathbb{E}}\left[\int_{0}^{T} \eta_{t}^{2} d t\right] .
\end{aligned}
$$


Proof. From Example 2.10, for each $j$,

$$
\hat{\mathbb{E}}\left[\xi_{j}\left(B_{t_{j+1}}-B_{t_{j}}\right) \mid \Omega_{t_{j}}\right]=\hat{\mathbb{E}}\left[-\xi_{j}\left(B_{t_{j+1}}-B_{t_{j}}\right) \mid \Omega_{t_{j}}\right]=0 .
$$

We have

$$
\begin{aligned}
\hat{\mathbb{E}}\left[\int_{0}^{T} \eta_{t} d B_{t}\right] & =\hat{\mathbb{E}}\left[\int_{0}^{t_{N-1}} \eta_{t} d B_{t}+\xi_{N-1}\left(B_{t_{N}}-B_{t_{N-1}}\right)\right] \\
& =\hat{\mathbb{E}}\left[\int_{0}^{t_{N-1}} \eta_{t} d B_{t}+\hat{\mathbb{E}}\left[\xi_{N-1}\left(B_{t_{N}}-B_{t_{N-1}}\right) \mid \Omega_{t_{N-1}}\right]\right] \\
& =\hat{\mathbb{E}}\left[\int_{0}^{t_{N-1}} \eta_{t} d B_{t}\right]
\end{aligned}
$$

Then we can repeat this procedure to obtain (3.4).

We now give the proof of (3.5). Firstly, from Example 2.10, we have

$$
\begin{aligned}
\hat{\mathbb{E}}\left[\left(\int_{0}^{T} \eta_{t} d B_{t}\right)^{2}\right]= & \hat{\mathbb{E}}\left[\left(\int_{0}^{t_{N-1}} \eta_{t} d B_{t}+\xi_{N-1}\left(B_{t_{N}}-B_{t_{N-1}}\right)\right)^{2}\right] \\
= & \hat{\mathbb{E}}\left[\left(\int_{0}^{t_{N-1}} \eta_{t} d B_{t}\right)^{2}+\xi_{N-1}^{2}\left(B_{t_{N}}-B_{t_{N-1}}\right)^{2}\right. \\
& \left.+2\left(\int_{0}^{t_{N-1}} \eta_{t} d B_{t}\right) \xi_{N-1}\left(B_{t_{N}}-B_{t_{N-1}}\right)\right] \\
= & \hat{\mathbb{E}}\left[\left(\int_{0}^{t_{N-1}} \eta_{t} d B_{t}\right)^{2}+\xi_{N-1}^{2}\left(B_{t_{N}}-B_{t_{N-1}}\right)^{2}\right] \\
= & \cdots=\hat{\mathbb{E}}\left[\sum_{i=0}^{N-1} \xi_{i}^{2}\left(B_{t_{i+1}}-B_{t_{i}}\right)^{2}\right] .
\end{aligned}
$$

Then, for each $i=0,1, \cdots, N-1$, we have

$$
\begin{aligned}
& \hat{\mathbb{E}}\left[\xi_{i}^{2}\left(B_{t_{i+1}}-B_{t_{i}}\right)^{2}-\bar{\sigma}^{2} \xi_{i}^{2}\left(t_{i+1}-t_{i}\right)\right] \\
= & \hat{\mathbb{E}}\left[\hat{\mathbb{E}}\left[\xi_{i}^{2}\left(B_{t_{i+1}}-B_{t_{i}}\right)^{2}-\bar{\sigma}^{2} \xi_{i}^{2}\left(t_{i+1}-t_{j}\right) \mid \Omega_{t_{i}}\right]\right] \\
= & \hat{\mathbb{E}}\left[\bar{\sigma}^{2} \xi_{i}^{2}\left(t_{i+1}-t_{i}\right)-\bar{\sigma}^{2} \xi_{i}^{2}\left(t_{i+1}-t_{i}\right)\right]=0 .
\end{aligned}
$$

Finally, we have

$$
\begin{aligned}
& \hat{\mathbb{E}}\left[\left(\int_{0}^{T} \eta_{t} d B_{t}\right)^{2}\right]=\hat{\mathbb{E}}\left[\sum_{i=0}^{N-1} \xi_{i}^{2}\left(B_{t_{i+1}}-B_{t_{i}}\right)^{2}\right] \\
\leq & \hat{\mathbb{E}}\left[\sum_{i=0}^{N-1} \xi_{i}^{2}\left(B_{t_{i+1}}-B_{t_{i}}\right)^{2}-\sum_{i=0}^{N-1} \bar{\sigma}^{2} \xi_{i}^{2}\left(t_{i+1}-t_{i}\right)\right]+\hat{\mathbb{E}}\left[\sum_{i=0}^{N-1} \bar{\sigma}^{2} \xi_{i}^{2}\left(t_{i+1}-t_{i}\right)\right] \\
\leq & \sum_{i=0}^{N-1} \hat{\mathbb{E}}\left[\xi_{i}^{2}\left(B_{t_{i+1}}-B_{t_{i}}\right)^{2}-\bar{\sigma}^{2} \xi_{i}^{2}\left(t_{i+1}-t_{i}\right)\right]+\hat{\mathbb{E}}\left[\sum_{i=0}^{N-1} \bar{\sigma}^{2} \xi_{i}^{2}\left(t_{i+1}-t_{i}\right)\right] \\
= & \hat{\mathbb{E}}\left[\sum_{i=0}^{N-1} \bar{\sigma}^{2} \xi_{i}^{2}\left(t_{i+1}-t_{i}\right)\right]=\bar{\sigma}^{2} \hat{\mathbb{E}}\left[\int_{0}^{T} \eta_{t}^{2} d t\right] .
\end{aligned}
$$


Definition 3.6 We define, for a fixed $\eta \in M_{G}^{2}(0, T)$, the stochastic integral

$$
\int_{0}^{T} \eta_{t} d B_{t}:=I(\eta)
$$

It is clear that (3.4) and (3.5) still hold for $\eta \in M_{G}^{2}(0, T)$.

We list some main properties of Itô's integral of $G$-Brownian motion. We denote, for some $0 \leq s \leq t \leq T$,

$$
\int_{s}^{t} \eta_{u} d B_{u}:=\int_{0}^{T} \mathbf{I}_{[s, t]}(u) \eta_{u} d B_{u}
$$

Proposition 3.7 Let $\eta, \theta \in M_{G}^{2}(0, T)$ and let $0 \leq s \leq r \leq t \leq T$. Then we have

(i) $\int_{s}^{t} \eta_{u} d B_{u}=\int_{s}^{r} \eta_{u} d B_{u}+\int_{r}^{t} \eta_{u} d B_{u}$.

(ii) $\int_{s}^{t}\left(\alpha \eta_{u}+\theta_{u}\right) d B_{u}=\alpha \int_{s}^{t} \eta_{u} d B_{u}+\int_{s}^{t} \theta_{u} d B_{u}$, if $\alpha$ is bounded and in $L_{G}^{1}\left(\Omega_{s}\right)$.

(iii) $\hat{\mathbb{E}}\left[X+\int_{r}^{T} \eta_{u} d B_{u} \mid \Omega_{s}\right]=\hat{\mathbb{E}}\left[X \mid \Omega_{s}\right] \quad$ for $X \in L_{G}^{1}(\Omega)$.

We now consider the multi-dimensional case. Let $G(\cdot): \mathbb{S}(d) \rightarrow \mathbb{R}$ be a given monotonic and sublinear function and let $\left(B_{t}\right)_{t>0}$ be a $d$-dimensional $G$ Brownian motion. For each fixed $\mathbf{a} \in \mathbb{R}^{d}$, we still use $B_{t}^{\mathbf{a}}:=\left\langle\mathbf{a}, B_{t}\right\rangle$. Then $\left(B_{t}^{\mathbf{a}}\right)_{t \geq 0}$ is a 1-dimensional $G_{\mathbf{a}}$-Brownian motion with $G_{\mathbf{a}}(\alpha)=\frac{1}{2}\left(\sigma_{\mathbf{a a}^{T}}^{2} \alpha^{+}-\right.$ $\left.\sigma_{-\mathbf{a a}^{T}}^{2} \alpha^{-}\right)$, where $\sigma_{\mathbf{a a}^{T}}^{2}=2 G\left(\mathbf{a a}^{T}\right)$ and $\sigma_{-\mathbf{a a}^{T}}^{2}=-2 G\left(-\mathbf{a a}^{T}\right)$. Similar to 1dimensional case, we can define Itô's integral by

$$
I(\eta):=\int_{0}^{T} \eta_{t} d B_{t}^{\mathbf{a}}, \text { for } \eta \in M_{G}^{2}(0, T) .
$$

We still have, for each $\eta \in M_{G}^{2}(0, T)$,

$$
\begin{aligned}
\hat{\mathbb{E}}\left[\int_{0}^{T} \eta_{t} d B_{t}^{\mathbf{a}}\right] & =0, \\
\hat{\mathbb{E}}\left[\left(\int_{0}^{T} \eta_{t} d B_{t}^{\mathbf{a}}\right)^{2}\right] & \leq \sigma_{\mathbf{a a}^{T}}^{2} \hat{\mathbb{E}}\left[\int_{0}^{T} \eta_{t}^{2} d t\right] .
\end{aligned}
$$

Furthermore, Proposition 3.7 still holds for the integral with respect to $B_{t}^{\mathbf{a}}$.

Exercise 3.8 Prove that, for a fixed $\eta \in M_{G}^{2}(0, T)$,

$$
\underline{\sigma}^{2} \hat{\mathbb{E}}\left[\int_{0}^{T} \eta_{t}^{2} d t\right] \leq \hat{\mathbb{E}}\left[\left(\int_{0}^{T} \eta_{t} d B_{t}\right)^{2}\right] \leq \bar{\sigma}^{2} \hat{\mathbb{E}}\left[\int_{0}^{T} \eta_{t}^{2} d t\right],
$$

where $\bar{\sigma}^{2}=\hat{\mathbb{E}}\left[B_{1}^{2}\right]$ and $\underline{\sigma}^{2}=-\hat{\mathbb{E}}\left[-B_{1}^{2}\right]$.

Exercise 3.9 Prove that, for each $\eta \in M_{G}^{p}(0, T)$, we have

$$
\hat{\mathbb{E}}\left[\int_{0}^{T}\left|\eta_{t}\right|^{p} d t\right] \leq \int_{0}^{T} \hat{\mathbb{E}}\left[\left|\eta_{t}\right|^{p}\right] d t .
$$




\section{$\S 4$ Quadratic Variation Process of $G$-Brownian Motion}

We first consider the quadratic variation process of 1-dimensional $G$-Brownian motion $\left(B_{t}\right)_{t \geq 0}$ with $B_{1} \stackrel{d}{=} N\left(\{0\} \times\left[\underline{\sigma}^{2}, \bar{\sigma}^{2}\right]\right)$. Let $\pi_{t}^{N}, N=1,2, \cdots$, be a sequence of partitions of $[0, t]$. We consider

$$
\begin{aligned}
B_{t}^{2} & =\sum_{j=0}^{N-1}\left(B_{t_{j+1}^{N}}^{2}-B_{t_{j}^{N}}^{2}\right) \\
& =\sum_{j=0}^{N-1} 2 B_{t_{j}^{N}}\left(B_{t_{j+1}^{N}}-B_{t_{j}^{N}}\right)+\sum_{j=0}^{N-1}\left(B_{t_{j+1}^{N}}-B_{t_{j}^{N}}\right)^{2} .
\end{aligned}
$$

As $\mu\left(\pi_{t}^{N}\right) \rightarrow 0$, the first term of the right side converges to $2 \int_{0}^{t} B_{s} d B_{s}$ in $L_{G}^{2}(\Omega)$. The second term must be convergent. We denote its limit by $\langle B\rangle_{t}$, i.e.,

$$
\langle B\rangle_{t}:=\lim _{\mu\left(\pi_{t}^{N}\right) \rightarrow 0} \sum_{j=0}^{N-1}\left(B_{t_{j+1}^{N}}-B_{t_{j}^{N}}\right)^{2}=B_{t}^{2}-2 \int_{0}^{t} B_{s} d B_{s} .
$$

By the above construction, $\left(\langle B\rangle_{t}\right)_{t \geq 0}$ is an increasing process with $\langle B\rangle_{0}=0$. We call it the quadratic variation process of the $G$-Brownian motion $B$. It characterizes the part of statistic uncertainty of $G$-Brownian motion. It is important to keep in mind that $\langle B\rangle_{t}$ is not a deterministic process unless $\underline{\sigma}=\bar{\sigma}$, i.e., when $\left(B_{t}\right)_{t \geq 0}$ is a classical Brownian motion. In fact we have the following lemma.

Lemma 4.1 For each $0 \leq s \leq t<\infty$, we have

$$
\begin{aligned}
\hat{\mathbb{E}}\left[\langle B\rangle_{t}-\langle B\rangle_{s} \mid \Omega_{s}\right] & =\bar{\sigma}^{2}(t-s), \\
\hat{\mathbb{E}}\left[-\left(\langle B\rangle_{t}-\langle B\rangle_{s}\right) \mid \Omega_{s}\right] & =-\underline{\sigma}^{2}(t-s) .
\end{aligned}
$$

Proof. By the definition of $\langle B\rangle$ and Proposition 3.7 (iii),

$$
\begin{aligned}
\hat{\mathbb{E}}\left[\langle B\rangle_{t}-\langle B\rangle_{s} \mid \Omega_{s}\right] & =\hat{\mathbb{E}}\left[B_{t}^{2}-B_{s}^{2}-2 \int_{s}^{t} B_{u} d B_{u} \mid \Omega_{s}\right] \\
& =\hat{\mathbb{E}}\left[B_{t}^{2}-B_{s}^{2} \mid \Omega_{s}\right]=\bar{\sigma}^{2}(t-s) .
\end{aligned}
$$

The last step follows from Example 2.11, We then have (4.7). The equality (4.8) can be proved analogously with the consideration of $\hat{\mathbb{E}}\left[-\left(B_{t}^{2}-B_{s}^{2}\right) \mid \Omega_{s}\right]=$ $-\underline{\sigma}^{2}(t-s)$.

A very interesting point of the quadratic variation process $\langle B\rangle$ is, just like the $G$-Brownian motion $B$ itself, the increment $\langle B\rangle_{s+t}-\langle B\rangle_{s}$ is independent from $\Omega_{s}$ and identically distributed with $\langle B\rangle_{t}$. In fact we have 
Lemma 4.2 For each fixed $s, t \geq 0,\langle B\rangle_{s+t}-\langle B\rangle_{s}$ is identically distributed with $\langle B\rangle_{t}$ and independent from $\Omega_{s}$.

Proof. The results follow directly from

$$
\begin{aligned}
\langle B\rangle_{s+t}-\langle B\rangle_{s} & =B_{s+t}^{2}-2 \int_{0}^{s+t} B_{r} d B_{r}-\left[B_{s}^{2}-2 \int_{0}^{s} B_{r} d B_{r}\right] \\
& =\left(B_{s+t}-B_{s}\right)^{2}-2 \int_{s}^{s+t}\left(B_{r}-B_{s}\right) d\left(B_{r}-B_{s}\right) \\
& =\left\langle B^{s}\right\rangle_{t}
\end{aligned}
$$

where $\left\langle B^{s}\right\rangle$ is the quadratic variation process of the $G$-Brownian motion $B_{t}^{s}=$ $B_{s+t}-B_{s}, t \geq 0$.

We now define the integral of a process $\eta \in M_{G}^{1}(0, T)$ with respect to $\langle B\rangle$. We first define a mapping:

$$
Q_{0, T}(\eta)=\int_{0}^{T} \eta_{t} d\langle B\rangle_{t}:=\sum_{j=0}^{N-1} \xi_{j}\left(\langle B\rangle_{t_{j+1}}-\langle B\rangle_{t_{j}}\right): M_{G}^{1,0}(0, T) \rightarrow L_{G}^{1}\left(\Omega_{T}\right) .
$$

Lemma 4.3 For each $\eta \in M_{G}^{1,0}(0, T)$,

$$
\hat{\mathbb{E}}\left[\left|Q_{0, T}(\eta)\right|\right] \leq \bar{\sigma}^{2} \hat{\mathbb{E}}\left[\int_{0}^{T}\left|\eta_{t}\right| d t\right] .
$$

Thus $Q_{0, T}: M_{G}^{1,0}(0, T) \rightarrow L_{G}^{1}\left(\Omega_{T}\right)$ is a continuous linear mapping. Consequently, $Q_{0, T}$ can be uniquely extended to $M_{G}^{1}(0, T)$. We still denote this mapping by

$$
\int_{0}^{T} \eta_{t} d\langle B\rangle_{t}:=Q_{0, T}(\eta) \text { for } \eta \in M_{G}^{1}(0, T)
$$

We still have

$$
\hat{\mathbb{E}}\left[\left|\int_{0}^{T} \eta_{t} d\langle B\rangle_{t}\right|\right] \leq \bar{\sigma}^{2} \hat{\mathbb{E}}\left[\int_{0}^{T}\left|\eta_{t}\right| d t\right] \quad \text { for } \eta \in M_{G}^{1}(0, T)
$$

Proof. Firstly, for each $j=1, \cdots, N-1$, we have

$$
\begin{aligned}
& \hat{\mathbb{E}}\left[\left|\xi_{j}\right|\left(\langle B\rangle_{t_{j+1}}-\langle B\rangle_{t_{j}}\right)-\bar{\sigma}^{2}\left|\xi_{j}\right|\left(t_{j+1}-t_{j}\right)\right] \\
= & \hat{\mathbb{E}}\left[\hat{\mathbb{E}}\left[\left|\xi_{j}\right|\left(\langle B\rangle_{t_{j+1}}-\langle B\rangle_{t_{j}}\right) \mid \Omega_{t_{j}}\right]-\bar{\sigma}^{2}\left|\xi_{j}\right|\left(t_{j+1}-t_{j}\right)\right] \\
= & \hat{\mathbb{E}}\left[\left|\xi_{j}\right| \bar{\sigma}^{2}\left(t_{j+1}-t_{j}\right)-\bar{\sigma}^{2}\left|\xi_{j}\right|\left(t_{j+1}-t_{j}\right)\right]=0 .
\end{aligned}
$$


Then (4.9) can be checked as follows:

$$
\begin{aligned}
& \hat{\mathbb{E}}\left[\left|\sum_{j=0}^{N-1} \xi_{j}\left(\langle B\rangle_{t_{j+1}}-\langle B\rangle_{t_{j}}\right)\right|\right] \leq \hat{\mathbb{E}}\left[\sum_{j=0}^{N-1}\left|\xi_{j}\right|\langle B\rangle_{t_{j+1}}-\langle B\rangle_{t_{j}}\right] \\
\leq & \hat{\mathbb{E}}\left[\sum_{j=0}^{N-1}\left|\xi_{j}\right|\left[\left(\langle B\rangle_{t_{j+1}}-\langle B\rangle_{t_{j}}\right)-\bar{\sigma}^{2}\left(t_{j+1}-t_{j}\right)\right]\right]+\hat{\mathbb{E}}\left[\bar{\sigma}^{2} \sum_{j=0}^{N-1}\left|\xi_{j}\right|\left(t_{j+1}-t_{j}\right)\right] \\
\leq & \sum_{j=0}^{N-1} \hat{\mathbb{E}}\left[\left|\xi_{j}\right|\left[\left(\langle B\rangle_{t_{j+1}}-\langle B\rangle_{t_{j}}\right)-\bar{\sigma}^{2}\left(t_{j+1}-t_{j}\right)\right]\right]+\hat{\mathbb{E}}\left[\bar{\sigma}^{2} \sum_{j=0}^{N-1}\left|\xi_{j}\right|\left(t_{j+1}-t_{j}\right)\right] \\
= & \hat{\mathbb{E}}\left[\bar{\sigma}^{2} \sum_{j=0}^{N-1}\left|\xi_{j}\right|\left(t_{j+1}-t_{j}\right)\right]=\bar{\sigma}^{2} \hat{\mathbb{E}}\left[\int_{0}^{T}\left|\eta_{t}\right| d t\right] .
\end{aligned}
$$

Proposition 4.4 Let $0 \leq s \leq t, \xi \in L_{G}^{2}\left(\Omega_{s}\right), X \in L_{G}^{1}(\Omega)$. Then

$$
\begin{aligned}
\hat{\mathbb{E}}\left[X+\xi\left(B_{t}^{2}-B_{s}^{2}\right)\right] & =\hat{\mathbb{E}}\left[X+\xi\left(B_{t}-B_{s}\right)^{2}\right] \\
& =\hat{\mathbb{E}}\left[X+\xi\left(\langle B\rangle_{t}-\langle B\rangle_{s}\right)\right] .
\end{aligned}
$$

Proof. By (4.6) and Proposition 3.7 (iii), we have

$$
\begin{aligned}
\hat{\mathbb{E}}\left[X+\xi\left(B_{t}^{2}-B_{s}^{2}\right)\right] & =\hat{\mathbb{E}}\left[X+\xi\left(\langle B\rangle_{t}-\langle B\rangle_{s}+2 \int_{s}^{t} B_{u} d B_{u}\right)\right] \\
& =\hat{\mathbb{E}}\left[X+\xi\left(\langle B\rangle_{t}-\langle B\rangle_{s}\right)\right] .
\end{aligned}
$$

We also have

$$
\begin{aligned}
\hat{\mathbb{E}}\left[X+\xi\left(B_{t}^{2}-B_{s}^{2}\right)\right] & =\hat{\mathbb{E}}\left[X+\xi\left(\left(B_{t}-B_{s}\right)^{2}+2\left(B_{t}-B_{s}\right) B_{s}\right)\right] \\
& =\hat{\mathbb{E}}\left[X+\xi\left(B_{t}-B_{s}\right)^{2}\right] .
\end{aligned}
$$

We have the following isometry.

Proposition 4.5 Let $\eta \in M_{G}^{2}(0, T)$. Then

$$
\hat{\mathbb{E}}\left[\left(\int_{0}^{T} \eta_{t} d B_{t}\right)^{2}\right]=\hat{\mathbb{E}}\left[\int_{0}^{T} \eta_{t}^{2} d\langle B\rangle_{t}\right] .
$$

Proof. We first consider $\eta \in M_{G}^{2,0}(0, T)$ of the form

$$
\eta_{t}(\omega)=\sum_{j=0}^{N-1} \xi_{j}(\omega) \mathbf{I}_{\left[t_{j}, t_{j+1}\right)}(t)
$$


and then $\int_{0}^{T} \eta_{t} d B_{t}=\sum_{j=0}^{N-1} \xi_{j}\left(B_{t_{j+1}}-B_{t_{j}}\right)$. From Proposition 3.7 we get

$$
\hat{\mathbb{E}}\left[X+2 \xi_{j}\left(B_{t_{j+1}}-B_{t_{j}}\right) \xi_{i}\left(B_{t_{i+1}}-B_{t_{i}}\right)\right]=\hat{\mathbb{E}}[X] \text { for } X \in L_{G}^{1}(\Omega), i \neq j .
$$

Thus

$$
\hat{\mathbb{E}}\left[\left(\int_{0}^{T} \eta_{t} d B_{t}\right)^{2}\right]=\hat{\mathbb{E}}\left[\left(\sum_{j=0}^{N-1} \xi_{j}\left(B_{t_{j+1}}-B_{t_{j}}\right)\right)^{2}\right]=\hat{\mathbb{E}}\left[\sum_{j=0}^{N-1} \xi_{j}^{2}\left(B_{t_{j+1}}-B_{t_{j}}\right)^{2}\right] .
$$

From this and Proposition 4.4, it follows that

$$
\hat{\mathbb{E}}\left[\left(\int_{0}^{T} \eta_{t} d B_{t}\right)^{2}\right]=\hat{\mathbb{E}}\left[\sum_{j=0}^{N-1} \xi_{j}^{2}\left(\langle B\rangle_{t_{j+1}}-\langle B\rangle_{t_{j}}\right)\right]=\hat{\mathbb{E}}\left[\int_{0}^{T} \eta_{t}^{2} d\langle B\rangle_{t}\right] .
$$

Thus (4.11) holds for $\eta \in M_{G}^{2,0}(0, T)$. We can continuously extend the above equality to the case $\eta \in M_{G}^{2}(0, T)$ and get (4.11).

We now consider the multi-dimensional case. Let $\left(B_{t}\right)_{t>0}$ be a $d$-dimensional $G$-Brownian motion. For each fixed $\mathbf{a} \in \mathbb{R}^{d},\left(B_{t}^{\mathbf{a}}\right)_{t \geq 0}$ is a 1-dimensional $G_{\mathbf{a}^{-}}$ Brownian motion. Similar to 1-dimensional case, we can define

$$
\left\langle B^{\mathbf{a}}\right\rangle_{t}:=\lim _{\mu\left(\pi_{t}^{N}\right) \rightarrow 0} \sum_{j=0}^{N-1}\left(B_{t_{j+1}^{N}}^{\mathbf{a}}-B_{t_{j}^{N}}^{\mathbf{a}}\right)^{2}=\left(B_{t}^{\mathbf{a}}\right)^{2}-2 \int_{0}^{t} B_{s}^{\mathbf{a}} d B_{s}^{\mathbf{a}},
$$

where $\left\langle B^{\mathbf{a}}\right\rangle$ is called the quadratic variation process of $B^{\mathbf{a}}$. The above results also hold for $\left\langle B^{\mathbf{a}}\right\rangle$. In particular,

$$
\hat{\mathbb{E}}\left[\left|\int_{0}^{T} \eta_{t} d\left\langle B^{\mathbf{a}}\right\rangle_{t}\right|\right] \leq \sigma_{\mathbf{a a}^{T}}^{2} \hat{\mathbb{E}}\left[\int_{0}^{T}\left|\eta_{t}\right| d t\right] \text { for } \eta \in M_{G}^{1}(0, T)
$$

and

$$
\hat{\mathbb{E}}\left[\left(\int_{0}^{T} \eta_{t} d B_{t}^{\mathbf{a}}\right)^{2}\right]=\hat{\mathbb{E}}\left[\int_{0}^{T} \eta_{t}^{2} d\left\langle B^{\mathbf{a}}\right\rangle_{t}\right] \text { for } \eta \in M_{G}^{2}(0, T) .
$$

Let $\mathbf{a}=\left(a_{1}, \cdots, a_{d}\right)^{T}$ and $\overline{\mathbf{a}}=\left(\bar{a}_{1}, \cdots, \bar{a}_{d}\right)^{T}$ be two given vectors in $\mathbb{R}^{d}$. We then have their quadratic variation processes of $\left\langle B^{\mathbf{a}}\right\rangle$ and $\left\langle B^{\overline{\mathbf{a}}}\right\rangle$. We can define their mutual variation process by

$$
\begin{aligned}
\left\langle B^{\mathbf{a}}, B^{\overline{\mathbf{a}}}\right\rangle_{t} & :=\frac{1}{4}\left[\left\langle B^{\mathbf{a}}+B^{\overline{\mathbf{a}}}\right\rangle_{t}-\left\langle B^{\mathbf{a}}-B^{\overline{\mathbf{a}}}\right\rangle_{t}\right] \\
& =\frac{1}{4}\left[\left\langle B^{\mathbf{a}+\overline{\mathbf{a}}}\right\rangle_{t}-\left\langle B^{\mathbf{a}-\overline{\mathbf{a}}}\right\rangle_{t}\right] .
\end{aligned}
$$

Since $\left\langle B^{\mathbf{a}-\overline{\mathbf{a}}}\right\rangle=\left\langle B^{\overline{\mathbf{a}}-\mathbf{a}}\right\rangle=\left\langle-B^{\mathbf{a}-\overline{\mathbf{a}}}\right\rangle$, we see that $\left\langle B^{\mathbf{a}}, B^{\overline{\mathbf{a}}}\right\rangle_{t}=\left\langle B^{\overline{\mathbf{a}}}, B^{\mathbf{a}}\right\rangle_{t}$. In particular, we have $\left\langle B^{\mathbf{a}}, B^{\mathbf{a}}\right\rangle=\left\langle B^{\mathbf{a}}\right\rangle$. Let $\pi_{t}^{N}, N=1,2, \cdots$, be a sequence of partitions of $[0, t]$. We observe that

$$
\sum_{k=0}^{N-1}\left(B_{t_{k+1}^{N}}^{\mathbf{a}}-B_{t_{k}^{N}}^{\mathbf{a}}\right)\left(B_{t_{k+1}^{N}}^{\overline{\overline{\mathbf{a}}}}-B_{t_{k}^{N}}^{\overline{\mathbf{a}}}\right)=\frac{1}{4} \sum_{k=0}^{N-1}\left[\left(B_{t_{k+1}}^{\mathbf{a}+\overline{\mathbf{a}}}-B_{t_{k}}^{\mathbf{a}+\overline{\mathbf{a}}}\right)^{2}-\left(B_{t_{k+1}}^{\mathbf{a}-\overline{\mathbf{a}}}-B_{t_{k}}^{\mathbf{a}-\overline{\mathbf{a}}}\right)^{2}\right] .
$$


Thus as $\mu\left(\pi_{t}^{N}\right) \rightarrow 0$ we have

$$
\lim _{N \rightarrow \infty} \sum_{k=0}^{N-1}\left(B_{t_{k+1}^{N}}^{\mathbf{a}}-B_{t_{k}^{N}}^{\mathbf{a}}\right)\left(B_{t_{k+1}^{N}}^{\overline{\overline{\mathbf{a}}}}-B_{t_{k}^{N}}^{\overline{\overline{\mathbf{a}}}}\right)=\left\langle B^{\mathbf{a}}, B^{\overline{\mathbf{a}}}\right\rangle_{t} .
$$

We also have

$$
\begin{aligned}
\left\langle B^{\mathbf{a}}, B^{\overline{\mathbf{a}}}\right\rangle_{t} & =\frac{1}{4}\left[\left\langle B^{\mathbf{a}+\overline{\mathbf{a}}}\right\rangle_{t}-\left\langle B^{\mathbf{a}-\overline{\mathbf{a}}}\right\rangle_{t}\right] \\
& =\frac{1}{4}\left[\left(B_{t}^{\mathbf{a}+\overline{\mathbf{a}}}\right)^{2}-2 \int_{0}^{t} B_{s}^{\mathbf{a}+\overline{\mathbf{a}}} d B_{s}^{\mathbf{a}+\overline{\mathbf{a}}}-\left(B_{t}^{\mathbf{a}-\overline{\mathbf{a}}}\right)^{2}+2 \int_{0}^{t} B_{s}^{\mathbf{a}-\overline{\mathbf{a}}} d B_{s}^{\mathbf{a}-\overline{\mathbf{a}}}\right] \\
& =B_{t}^{\mathbf{a}} B_{t}^{\overline{\mathbf{a}}}-\int_{0}^{t} B_{s}^{\mathbf{a}} d B_{s}^{\overline{\mathbf{a}}}-\int_{0}^{t} B_{s}^{\overline{\mathbf{a}}} d B_{s}^{\mathbf{a}}
\end{aligned}
$$

Now for each $\eta \in M_{G}^{1}(0, T)$, we can consistently define

$$
\int_{0}^{T} \eta_{t} d\left\langle B^{\mathbf{a}}, B^{\overline{\mathbf{a}}}\right\rangle_{t}=\frac{1}{4} \int_{0}^{T} \eta_{t} d\left\langle B^{\mathbf{a}+\overline{\mathbf{a}}}\right\rangle_{t}-\frac{1}{4} \int_{0}^{T} \eta_{t} d\left\langle B^{\mathbf{a}-\overline{\mathbf{a}}}\right\rangle_{t} .
$$

Lemma 4.6 Let $\eta^{N} \in M_{G}^{2,0}(0, T), N=1,2, \cdots$, be of the form

$$
\eta_{t}^{N}(\omega)=\sum_{k=0}^{N-1} \xi_{k}^{N}(\omega) \mathbf{I}_{\left[t_{k}^{N}, t_{k+1}^{N}\right)}(t)
$$

with $\mu\left(\pi_{T}^{N}\right) \rightarrow 0$ and $\eta^{N} \rightarrow \eta$ in $M_{G}^{2}(0, T)$, as $N \rightarrow \infty$. Then we have the following convergence in $L_{G}^{2}\left(\Omega_{T}\right)$ :

$$
\sum_{k=0}^{N-1} \xi_{k}^{N}\left(B_{t_{k+1}^{N}}^{\mathbf{a}}-B_{t_{k}^{N}}^{\mathbf{a}}\right)\left(B_{t_{k+1}^{N}}^{\overline{\mathbf{a}}}-B_{t_{k}^{N}}^{\overline{\mathbf{a}}}\right) \rightarrow \int_{0}^{T} \eta_{t} d\left\langle B^{\mathbf{a}}, B^{\overline{\mathbf{a}}}\right\rangle_{t} .
$$

Proof. Since

$$
\begin{aligned}
\left\langle B^{\mathbf{a}}, B^{\overline{\mathbf{a}}}\right\rangle_{t_{k+1}^{N}}-\left\langle B^{\mathbf{a}}, B^{\overline{\mathbf{a}}}\right\rangle_{t_{k}^{N}}= & \left(B_{t_{k+1}^{N}}^{\mathbf{a}}-B_{t_{k}^{N}}^{\mathbf{a}}\right)\left(B_{t_{k+1}^{N}}^{\overline{\mathbf{a}}}-B_{t_{k}^{N}}^{\overline{\mathbf{a}}}\right) \\
& -\int_{t_{k}^{N}}^{t_{k+1}^{N}}\left(B_{s}^{\mathbf{a}}-B_{t_{k}^{N}}^{\mathbf{a}}\right) d B_{s}^{\overline{\mathbf{a}}}-\int_{t_{k}^{N}}^{t_{k+1}^{N}}\left(B_{s}^{\overline{\mathbf{a}}}-B_{t_{k}^{N}}^{\overline{\overline{\mathbf{a}}}}\right) d B_{s}^{\mathbf{a}},
\end{aligned}
$$

we only need to prove

$$
\hat{\mathbb{E}}\left[\sum_{k=0}^{N-1}\left(\xi_{k}^{N}\right)^{2}\left(\int_{t_{k}^{N}}^{t_{k+1}^{N}}\left(B_{s}^{\mathbf{a}}-B_{t_{k}^{N}}^{\mathbf{a}}\right) d B_{s}^{\overline{\mathbf{a}}}\right)^{2}\right] \rightarrow 0 .
$$

For each $k=1, \cdots, N-1$, we have

$$
\begin{aligned}
& \hat{\mathbb{E}}\left[\left(\xi_{k}^{N}\right)^{2}\left(\int_{t_{k}^{N}}^{t_{k+1}^{N}}\left(B_{s}^{\mathbf{a}}-B_{t_{k}^{N}}^{\mathbf{a}}\right) d B_{s}^{\overline{\mathbf{a}}}\right)^{2}-C\left(\xi_{k}^{N}\right)^{2}\left(t_{k+1}^{N}-t_{k}^{N}\right)^{2}\right] \\
= & \hat{\mathbb{E}}\left[\hat{\mathbb{E}}\left[\left(\xi_{k}^{N}\right)^{2}\left(\int_{t_{k}^{N}}^{t_{k+1}^{N}}\left(B_{s}^{\mathbf{a}}-B_{t_{k}^{N}}^{\mathbf{a}}\right) d B_{s}^{\overline{\mathbf{a}}}\right)^{2} \mid \Omega_{t_{k}^{N}}\right]-C\left(\xi_{k}^{N}\right)^{2}\left(t_{k+1}^{N}-t_{k}^{N}\right)^{2}\right] \\
\leq & \hat{\mathbb{E}}\left[C\left(\xi_{k}^{N}\right)^{2}\left(t_{k+1}^{N}-t_{k}^{N}\right)^{2}-C\left(\xi_{k}^{N}\right)^{2}\left(t_{k+1}^{N}-t_{k}^{N}\right)^{2}\right]=0,
\end{aligned}
$$


where $C=\bar{\sigma}_{\mathbf{a a}^{T}}^{2} \bar{\sigma}_{\overline{\mathbf{a}} \overline{\mathbf{T}}^{T}}^{2} / 2$.

Thus we have

$$
\begin{aligned}
& \hat{\mathbb{E}}\left[\sum_{k=0}^{N-1}\left(\xi_{k}^{N}\right)^{2}\left(\int_{t_{k}^{N}}^{t_{k+1}^{N}}\left(B_{s}^{\mathbf{a}}-B_{t_{k}^{N}}^{\mathbf{a}}\right) d B_{s}^{\overline{\mathbf{a}}}\right)^{2}\right] \\
& \leq \hat{\mathbb{E}}\left[\sum_{k=0}^{N-1}\left(\xi_{k}^{N}\right)^{2}\left[\left(\int_{t_{k}^{N}}^{t_{k+1}^{N}}\left(B_{s}^{\mathbf{a}}-B_{t_{k}^{N}}^{\mathbf{a}}\right) d B_{s}^{\overline{\mathbf{a}}}\right)^{2}-C\left(t_{k+1}^{N}-t_{k}^{N}\right)^{2}\right]\right] \\
& \quad+\hat{\mathbb{E}}\left[\sum_{k=0}^{N-1} C\left(\xi_{k}^{N}\right)^{2}\left(t_{k+1}^{N}-t_{k}^{N}\right)^{2}\right] \\
& \leq \sum_{k=0}^{N-1} \hat{\mathbb{E}}\left[\left(\xi_{k}^{N}\right)^{2}\left[\left(\int_{t_{k}^{N}}^{t_{k+1}^{N}}\left(B_{s}^{\mathbf{a}}-B_{t_{k}^{N}}^{\mathbf{a}}\right) d B_{s}^{\overline{\mathbf{a}}}\right)^{2}-C\left(t_{k+1}^{N}-t_{k}^{N}\right)^{2}\right]\right] \\
& \quad+\hat{\mathbb{E}}\left[\sum_{k=0}^{N-1} C\left(\xi_{k}^{N}\right)^{2}\left(t_{k+1}^{N}-t_{k}^{N}\right)^{2}\right] \\
& \leq \hat{\mathbb{E}}\left[\sum_{k=0}^{N-1} C\left(\xi_{k}^{N}\right)^{2}\left(t_{k+1}^{N}-t_{k}^{N}\right)^{2}\right] \leq C \mu\left(\pi_{T}^{N}\right) \hat{\mathbb{E}}\left[\int_{0}^{T}\left|\eta_{t}^{N}\right|^{2} d t\right],
\end{aligned}
$$

As $\mu\left(\pi_{T}^{N}\right) \rightarrow 0$, the proof is complete.

Exercise 4.7 Let $B_{t}$ be a 1-dimensional G-Brownian motion and $\varphi$ be a bounded and Lipschitz function on $\mathbb{R}$. Show that

$$
\lim _{N \rightarrow \infty} \hat{\mathbb{E}}\left[\left|\sum_{k=0}^{N-1} \varphi\left(B_{t_{k}^{N}}\right)\left[\left(B_{t_{k+1}^{N}}-B_{t_{k}^{N}}\right)^{2}-\left(\langle B\rangle_{t_{k+1}^{N}}-\langle B\rangle_{t_{k}^{N}}\right)\right]\right|\right]=0,
$$

where $t_{k}^{N}=k T / N, k=0,2, \cdots, N-1$.

Exercise 4.8 Prove that, for a fixed $\eta \in M_{G}^{1}(0, T)$,

$$
\underline{\sigma}^{2} \hat{\mathbb{E}}\left[\int_{0}^{T}\left|\eta_{t}\right| d t\right] \leq \hat{\mathbb{E}}\left[\int_{0}^{T}\left|\eta_{t}\right| d\langle B\rangle_{t}\right] \leq \bar{\sigma}^{2} \hat{\mathbb{E}}\left[\int_{0}^{T}\left|\eta_{t}\right| d t\right],
$$

where $\bar{\sigma}^{2}=\hat{\mathbb{E}}\left[B_{1}^{2}\right]$ and $\underline{\sigma}^{2}=-\hat{\mathbb{E}}\left[-B_{1}^{2}\right]$.

\section{$\S 5$ The Distribution of $\langle B\rangle$}

In this section, we first consider the 1-dimensional $G$-Brownian motion $\left(B_{t}\right)_{t \geq 0}$ with $B_{1} \stackrel{d}{=} N\left(\{0\} \times\left[\underline{\sigma}^{2}, \bar{\sigma}^{2}\right]\right)$.

The quadratic variation process $\langle B\rangle$ of $G$-Brownian motion $B$ is a very interesting process. We have seen that the $G$-Brownian motion $B$ is a typical process with variance uncertainty but without mean-uncertainty. In fact, $\langle B\rangle$ is 
concentrated all uncertainty of the $G$-Brownian motion $B$. Moreover, $\langle B\rangle$ itself is a typical process with mean-uncertainty. This fact will be applied to measure the mean-uncertainty of risk positions.

Lemma 5.1 We have

$$
\hat{\mathbb{E}}\left[\langle B\rangle_{t}^{2}\right] \leq 10 \bar{\sigma}^{4} t^{2}
$$

Proof. Indeed,

$$
\begin{aligned}
\hat{\mathbb{E}}\left[\langle B\rangle_{t}^{2}\right] & =\hat{\mathbb{E}}\left[\left(B_{t}^{2}-2 \int_{0}^{t} B_{u} d B_{u}\right)^{2}\right] \\
& \leq 2 \hat{\mathbb{E}}\left[B_{t}^{4}\right]+8 \hat{\mathbb{E}}\left[\left(\int_{0}^{t} B_{u} d B_{u}\right)^{2}\right] \\
& \leq 6 \bar{\sigma}^{4} t^{2}+8 \bar{\sigma}^{2} \hat{\mathbb{E}}\left[\int_{0}^{t} B_{u}{ }^{2} d u\right] \\
& \leq 6 \bar{\sigma}^{4} t^{2}+8 \bar{\sigma}^{2} \int_{0}^{t} \hat{\mathbb{E}}\left[B_{u}{ }^{2}\right] d u \\
& =10 \bar{\sigma}^{4} t^{2} .
\end{aligned}
$$

Proposition 5.2 Let $\left(b_{t}\right)_{t \geq 0}$ be a process on a sublinear expectation space $(\Omega, \mathcal{H}, \hat{\mathbb{E}})$ such that

(i) $b_{0}=0$;

(ii) For each $t, s \geq 0, b_{t+s}-b_{t}$ is identically distributed with $b_{s}$ and independent from $\left(b_{t_{1}}, b_{t_{2}}, \cdots, b_{t_{n}}\right)$ for each $n \in \mathbb{N}$ and $0 \leq t_{1}, \cdots, t_{n} \leq t$;

(iii) $\lim _{t \downarrow 0} \hat{\mathbb{E}}\left[b_{t}^{2}\right] t^{-1}=0$.

Then $b_{t}$ is $N([\underline{\mu} t, \bar{\mu} t] \times\{0\})$-distributed with $\bar{\mu}=\hat{\mathbb{E}}\left[b_{1}\right]$ and $\underline{\mu}=-\hat{\mathbb{E}}\left[-b_{1}\right]$.

Proof. We first prove that

$$
\hat{\mathbb{E}}\left[b_{t}\right]=\bar{\mu} t \text { and }-\hat{\mathbb{E}}\left[-b_{t}\right]=\underline{\mu} t .
$$

We set $\varphi(t):=\hat{\mathbb{E}}\left[b_{t}\right]$. Then $\varphi(0)=0$ and $\lim _{t \downarrow 0} \varphi(t)=0$. Since for each $t, s \geq 0$,

$$
\begin{aligned}
\varphi(t+s) & =\hat{\mathbb{E}}\left[b_{t+s}\right]=\hat{\mathbb{E}}\left[\left(b_{t+s}-b_{s}\right)+b_{s}\right] \\
& =\varphi(t)+\varphi(s) .
\end{aligned}
$$

Thus $\varphi(t)$ is linear and uniformly continuous in $t$, which means that $\hat{\mathbb{E}}\left[b_{t}\right]=\bar{\mu} t$. Similarly $-\hat{\mathbb{E}}\left[-b_{t}\right]=\mu t$.

We now prove that $b_{t}$ is $N([\mu t, \bar{\mu} t] \times\{0\})$-distributed. By Exercise 1.17 in Chap $\amalg$, we just need to prove that for each fixed $\varphi \in C_{b . L i p}(\mathbb{R})$, the function

$$
u(t, x):=\hat{\mathbb{E}}\left[\varphi\left(x+b_{t}\right)\right], \quad(t, x) \in[0, \infty) \times \mathbb{R}
$$


is the viscosity solution of the following parabolic PDE:

$$
\partial_{t} u-g\left(\partial_{x} u\right)=0,\left.\quad u\right|_{t=0}=\varphi
$$

with $g(a)=\bar{\mu} a^{+}-\underline{\mu} a^{-}$

We first prove that $u$ is Lipschitz in $x$ and $\frac{1}{2}$-Hölder continuous in $t$. In fact, for each fixed $t, u(t, \cdot) \in C_{b . \text { Lip }}(\mathbb{R})$ since

$$
\begin{aligned}
\left|\hat{\mathbb{E}}\left[\varphi\left(x+b_{t}\right)\right]-\hat{\mathbb{E}}\left[\varphi\left(y+b_{t}\right)\right]\right| & \leq \hat{\mathbb{E}}\left[\left|\varphi\left(x+b_{t}\right)-\varphi\left(y+b_{t}\right)\right|\right] \\
& \leq C|x-y| .
\end{aligned}
$$

For each $\delta \in[0, t]$, since $b_{t}-b_{\delta}$ is independent from $b_{\delta}$, we have

$$
\begin{aligned}
u(t, x) & =\hat{\mathbb{E}}\left[\varphi\left(x+b_{\delta}+\left(b_{t}-b_{\delta}\right)\right]\right. \\
& =\hat{\mathbb{E}}\left[\hat{\mathbb{E}}\left[\varphi\left(y+\left(b_{t}-b_{\delta}\right)\right)\right]_{y=x+b_{\delta}}\right],
\end{aligned}
$$

hence

$$
u(t, x)=\hat{\mathbb{E}}\left[u\left(t-\delta, x+b_{\delta}\right)\right] .
$$

Thus

$$
\begin{aligned}
|u(t, x)-u(t-\delta, x)| & =\left|\hat{\mathbb{E}}\left[u\left(t-\delta, x+b_{\delta}\right)-u(t-\delta, x)\right]\right| \\
& \leq \hat{\mathbb{E}}\left[\left|u\left(t-\delta, x+b_{\delta}\right)-u(t-\delta, x)\right|\right] \\
& \leq \hat{\mathbb{E}}\left[C\left|b_{\delta}\right|\right] \leq C_{1} \sqrt{\delta} .
\end{aligned}
$$

To prove that $u$ is a viscosity solution of the PDE (5.13), we fix a point $(t, x) \in(0, \infty) \times \mathbb{R}$ and let $v \in C_{b}^{2,2}([0, \infty) \times \mathbb{R})$ be such that $v \geq u$ and $v(t, x)=u(t, x)$. From (5.14), we have

$$
v(t, x)=\hat{\mathbb{E}}\left[u\left(t-\delta, x+b_{\delta}\right)\right] \leq \hat{\mathbb{E}}\left[v\left(t-\delta, x+b_{\delta}\right)\right] .
$$

Therefore, by Taylor's expansion,

$$
\begin{aligned}
0 & \leq \hat{\mathbb{E}}\left[v\left(t-\delta, x+b_{\delta}\right)-v(t, x)\right] \\
& =\hat{\mathbb{E}}\left[v\left(t-\delta, x+b_{\delta}\right)-v\left(t, x+b_{\delta}\right)+\left(v\left(t, x+b_{\delta}\right)-v(t, x)\right)\right] \\
& =\hat{\mathbb{E}}\left[-\partial_{t} v(t, x) \delta+\partial_{x} v(t, x) b_{\delta}+I_{\delta}\right] \\
& \leq-\partial_{t} v(t, x) \delta+\hat{\mathbb{E}}\left[\partial_{x} v(t, x) b_{\delta}\right]+\hat{\mathbb{E}}\left[I_{\delta}\right] \\
& =-\partial_{t} v(t, x) \delta+g\left(\partial_{x} v(t, x)\right) \delta+\hat{\mathbb{E}}\left[I_{\delta}\right],
\end{aligned}
$$

where

$$
\begin{aligned}
I_{\delta}= & \delta \int_{0}^{1}\left[-\partial_{t} v\left(t-\beta \delta, x+b_{\delta}\right)+\partial_{t} v(t, x)\right] d \beta \\
& +b_{\delta} \int_{0}^{1}\left[\partial_{x} v\left(t, x+\beta b_{\delta}\right)-\partial_{x} v(t, x)\right] d \beta .
\end{aligned}
$$


With the assumption that $\lim _{t \downarrow 0} \hat{\mathbb{E}}\left[b_{t}^{2}\right] t^{-1}=0$, we can check that

$$
\lim _{\delta \downarrow 0} \hat{\mathbb{E}}\left[\left|I_{\delta}\right|\right] \delta^{-1}=0
$$

from which we get $\partial_{t} v(t, x)-g\left(\partial_{x} v(t, x)\right) \leq 0$, hence $u$ is a viscosity subsolution of (5.13). We can analogously prove that $u$ is also a viscosity supersolution. It follows that $b_{t}$ is $N([\mu t, \bar{\mu} t] \times\{0\})$-distributed. The proof is complete.

It is clear that $\langle\bar{B}\rangle$ satisfies all the conditions in the Proposition [5.2, thus we immediately have

Theorem 5.3 $\langle B\rangle_{t}$ is $N\left(\left[\underline{\sigma}^{2} t, \bar{\sigma}^{2} t\right] \times\{0\}\right)$-distributed, i.e., for each $\varphi \in C_{l . L i p}(\mathbb{R})$,

$$
\hat{\mathbb{E}}\left[\varphi\left(\langle B\rangle_{t}\right)\right]=\sup _{\underline{\sigma}^{2} \leq v \leq \bar{\sigma}^{2}} \varphi(v t) .
$$

Corollary 5.4 For each $0 \leq t \leq T<\infty$, we have

$$
\underline{\sigma}^{2}(T-t) \leq\langle B\rangle_{T}-\langle B\rangle_{t} \leq \bar{\sigma}^{2}(T-t) \text { in } L_{G}^{1}(\Omega) .
$$

Proof. It is a direct consequence of

$$
\hat{\mathbb{E}}\left[\left(\langle B\rangle_{T}-\langle B\rangle_{t}-\bar{\sigma}^{2}(T-t)\right)^{+}\right]=\sup _{\underline{\sigma}^{2} \leq v \leq \bar{\sigma}^{2}}\left(v-\bar{\sigma}^{2}\right)^{+}(T-t)=0
$$

and

$$
\hat{\mathbb{E}}\left[\left(\langle B\rangle_{T}-\langle B\rangle_{t}-\underline{\sigma}^{2}(T-t)\right)^{-}\right]=\sup _{\underline{\sigma}^{2} \leq v \leq \bar{\sigma}^{2}}\left(v-\underline{\sigma}^{2}\right)^{-}(T-t)=0 .
$$

Corollary 5.5 We have, for each $t, s \geq 0, n \in \mathbb{N}$,

$$
\hat{\mathbb{E}}\left[\left(\langle B\rangle_{t+s}-\langle B\rangle_{s}\right)^{n} \mid \Omega_{s}\right]=\hat{\mathbb{E}}\left[\langle B\rangle_{t}^{n}\right]=\bar{\sigma}^{2 n} t^{n}
$$

and

$$
\hat{\mathbb{E}}\left[-\left(\langle B\rangle_{t+s}-\langle B\rangle_{s}\right)^{n} \mid \Omega_{s}\right]=\hat{\mathbb{E}}\left[-\langle B\rangle_{t}^{n}\right]=-\underline{\sigma}^{2 n} t^{n} .
$$

We now consider the multi-dimensional case. For notational simplicity, we denote by $B^{i}:=B^{\mathbf{e}_{i}}$ the $i$-th coordinate of the $G$-Brownian motion $B$, under a given orthonormal basis $\left(\mathbf{e}_{1}, \cdots, \mathbf{e}_{d}\right)$ of $\mathbb{R}^{d}$. We denote

$$
\left(\langle B\rangle_{t}\right)_{i j}:=\left\langle B^{i}, B^{j}\right\rangle_{t} .
$$

Then $\langle B\rangle_{t}, t \geq 0$, is an $\mathbb{S}(d)$-valued process. Since

$$
\hat{\mathbb{E}}\left[\left\langle A B_{t}, B_{t}\right\rangle\right]=2 G(A) t \text { for } A \in \mathbb{S}(d),
$$


we have

$$
\begin{aligned}
\hat{\mathbb{E}}\left[\left(\langle B\rangle_{t}, A\right)\right] & =\hat{\mathbb{E}}\left[\sum_{i, j=1}^{d} a_{i j}\left\langle B^{i}, B^{j}\right\rangle_{t}\right] \\
& =\hat{\mathbb{E}}\left[\sum_{i, j=1}^{d} a_{i j}\left(B_{t}^{i} B_{t}^{j}-\int_{0}^{t} B_{s}^{i} d B_{s}^{j}-\int_{0}^{t} B_{s}^{j} d B_{s}^{i}\right)\right] \\
& =\hat{\mathbb{E}}\left[\sum_{i, j=1}^{d} a_{i j} B_{t}^{i} B_{t}^{j}\right]=2 G(A) t \text { for } A \in \mathbb{S}(d),
\end{aligned}
$$

where $\left(a_{i j}\right)_{i, j=1}^{d}=A$.

Now we set, for each $\varphi \in C_{l . L i p}(\mathbb{S}(d))$,

$$
v(t, X):=\hat{\mathbb{E}}\left[\varphi\left(X+\langle B\rangle_{t}\right)\right], \quad(t, X) \in[0, \infty) \times \mathbb{S}(d) .
$$

Let $\Sigma \subset \mathbb{S}_{+}(d)$ be the bounded, convex and closed subset such that

$$
G(A)=\frac{1}{2} \sup _{B \in \Sigma}(A, B), \quad A \in \mathbb{S}(d) .
$$

Proposition 5.6 The function $v$ solves the following first order PDE:

$$
\partial_{t} v-2 G(D v)=0,\left.v\right|_{t=0}=\varphi
$$

where $D v=\left(\partial_{x_{i j}} v\right)_{i, j=1}^{d}$. We also have

$$
v(t, X)=\sup _{\Lambda \in \Sigma} \varphi(X+t \Lambda)
$$

Sketch of the Proof. We have

$$
\begin{aligned}
v(t+\delta, X) & =\hat{\mathbb{E}}\left[\varphi\left(X+\langle B\rangle_{\delta}+\langle B\rangle_{t+\delta}-\langle B\rangle_{\delta}\right)\right] \\
& =\hat{\mathbb{E}}\left[v\left(t, X+\langle B\rangle_{\delta}\right)\right] .
\end{aligned}
$$

The rest part of the proof is similar to the 1-dimensional case.

Corollary 5.7 We have

$$
\langle B\rangle_{t} \in t \Sigma:=\{t \times \gamma: \gamma \in \Sigma\}
$$

or equivalently, $d_{t \Sigma}\left(\langle B\rangle_{t}\right)=0$, where $d_{U}(X)=\inf \{\sqrt{(X-Y, X-Y)}: Y \in U\}$.

Proof. Since

$$
\hat{\mathbb{E}}\left[d_{t \Sigma}\left(\langle B\rangle_{t}\right)\right]=\sup _{\Lambda \in \Sigma} d_{t \Sigma}(t \Lambda)=0
$$

it follows that $d_{t \Sigma}\left(\langle B\rangle_{t}\right)=0$.

Exercise 5.8 Complete the proof of Proposition 5.6. 


\section{$\S 6 \quad G-$ Itô's Formula}

In this section, we give Itô's formula for a " $G$-Itô process" $X$. For simplicity, we first consider the case of the function $\Phi$ is sufficiently regular.

Lemma 6.1 Let $\Phi \in C^{2}\left(\mathbb{R}^{n}\right)$ with $\partial_{x^{\nu}} \Phi, \partial_{x^{\mu} x^{\nu}}^{2} \Phi \in C_{b . \text { Lip }}\left(\mathbb{R}^{n}\right)$ for $\mu, \nu=$ $1, \cdots, n$. Let $s \in[0, T]$ be fixed and let $X=\left(X^{1}, \cdots, X^{n}\right)^{T}$ be an $n$-dimensional process on $[s, T]$ of the form

$$
X_{t}^{\nu}=X_{s}^{\nu}+\alpha^{\nu}(t-s)+\eta^{\nu i j}\left(\left\langle B^{i}, B^{j}\right\rangle_{t}-\left\langle B^{i}, B^{j}\right\rangle_{s}\right)+\beta^{\nu j}\left(B_{t}^{j}-B_{s}^{j}\right),
$$

where, for $\nu=1, \cdots, n, i, j=1, \cdots, d, \alpha^{\nu}, \eta^{\nu i j}$ and $\beta^{\nu j}$ are bounded elements in $L_{G}^{2}\left(\Omega_{s}\right)$ and $X_{s}=\left(X_{s}^{1}, \cdots, X_{s}^{n}\right)^{T}$ is a given random vector in $L_{G}^{2}\left(\Omega_{s}\right)$. Then we have, in $L_{G}^{2}\left(\Omega_{t}\right)$,

$$
\begin{aligned}
\Phi\left(X_{t}\right)-\Phi\left(X_{s}\right)= & \int_{s}^{t} \partial_{x^{\nu}} \Phi\left(X_{u}\right) \beta^{\nu j} d B_{u}^{j}+\int_{s}^{t} \partial_{x^{\nu}} \Phi\left(X_{u}\right) \alpha^{\nu} d u \\
& +\int_{s}^{t}\left[\partial_{x^{\nu}} \Phi\left(X_{u}\right) \eta^{\nu i j}+\frac{1}{2} \partial_{x^{\mu} x^{\nu}}^{2} \Phi\left(X_{u}\right) \beta^{\mu i} \beta^{\nu j}\right] d\left\langle B^{i}, B^{j}\right\rangle_{u} .
\end{aligned}
$$

Here we use the, i.e., the above repeated indices $\mu, \nu, i$ and $j$ imply the summation.

Proof. For each positive integer $N$, we set $\delta=(t-s) / N$ and take the partition

$$
\pi_{[s, t]}^{N}=\left\{t_{0}^{N}, t_{1}^{N}, \cdots, t_{N}^{N}\right\}=\{s, s+\delta, \cdots, s+N \delta=t\} .
$$

We have

$$
\begin{aligned}
\Phi\left(X_{t}\right)-\Phi\left(X_{s}\right)= & \sum_{k=0}^{N-1}\left[\Phi\left(X_{t_{k+1}^{N}}\right)-\Phi\left(X_{t_{k}^{N}}\right)\right] \\
= & \sum_{k=0}^{N-1}\left\{\partial_{x^{\nu}} \Phi\left(X_{t_{k}^{N}}\right)\left(X_{t_{k+1}^{N}}^{\nu}-X_{t_{k}^{N}}^{\nu}\right)\right. \\
& \left.+\frac{1}{2}\left[\partial_{x^{\mu} x^{\nu}}^{2} \Phi\left(X_{t_{k}^{N}}\right)\left(X_{t_{k+1}^{N}}^{\mu}-X_{t_{k}^{N}}^{\mu}\right)\left(X_{t_{k+1}^{N}}^{\nu}-X_{t_{k}^{N}}^{\nu}\right)+\eta_{k}^{N}\right]\right\}
\end{aligned}
$$

where

$\eta_{k}^{N}=\left[\partial_{x^{\mu} x^{\nu}}^{2} \Phi\left(X_{t_{k}^{N}}+\theta_{k}\left(X_{t_{k+1}^{N}}-X_{t_{k}^{N}}\right)\right)-\partial_{x^{\mu} x^{\nu}}^{2} \Phi\left(X_{t_{k}^{N}}\right)\right]\left(X_{t_{k+1}^{N}}^{\mu}-X_{t_{k}^{N}}^{\mu}\right)\left(X_{t_{k+1}^{N}}^{\nu}-X_{t_{k}^{N}}^{\nu}\right)$

with $\theta_{k} \in[0,1]$. We have

$$
\begin{aligned}
\hat{\mathbb{E}}\left[\left|\eta_{k}^{N}\right|^{2}\right]= & \hat{\mathbb{E}}\left[\mid\left[\partial_{x^{\mu} x^{\nu}}^{2} \Phi\left(X_{t_{k}^{N}}+\theta_{k}\left(X_{t_{k+1}^{N}}-X_{t_{k}^{N}}\right)\right)-\partial_{x^{\mu} x^{\nu}}^{2} \Phi\left(X_{t_{k}^{N}}\right)\right]\right. \\
& \left.\times\left.\left(X_{t_{k+1}^{N}}^{\mu}-X_{t_{k}^{N}}^{\mu}\right)\left(X_{t_{k+1}^{N}}^{\nu}-X_{t_{k}^{N}}^{\nu}\right)\right|^{2}\right] \\
\leq & c \hat{\mathbb{E}}\left[\left|X_{t_{k+1}^{N}}-X_{t_{k}^{N}}\right|^{6}\right] \leq C\left[\delta^{6}+\delta^{3}\right]
\end{aligned}
$$


where $c$ is the Lipschitz constant of $\left\{\partial_{x^{\mu} x^{\nu}}^{2} \Phi\right\}_{\mu, \nu=1}^{n}$ and $C$ is a constant independent of $k$. Thus

$$
\hat{\mathbb{E}}\left[\left|\sum_{k=0}^{N-1} \eta_{k}^{N}\right|^{2}\right] \leq N \sum_{k=0}^{N-1} \hat{\mathbb{E}}\left[\left|\eta_{k}^{N}\right|^{2}\right] \rightarrow 0 .
$$

The rest terms in the summation of the right side of (6.19) are $\xi_{t}^{N}+\zeta_{t}^{N}$ with

$$
\begin{aligned}
\xi_{t}^{N}= & \sum_{k=0}^{N-1}\left\{\partial _ { x ^ { \nu } } \Phi ( X _ { t _ { k } ^ { N } } ) \left[\alpha^{\nu}\left(t_{k+1}^{N}-t_{k}^{N}\right)+\eta^{\nu i j}\left(\left\langle B^{i}, B^{j}\right\rangle_{t_{k+1}^{N}}-\left\langle B^{i}, B^{j}\right\rangle_{t_{k}^{N}}\right)\right.\right. \\
& \left.\left.+\beta^{\nu j}\left(B_{t_{k+1}^{N}}^{j}-B_{t_{k}^{N}}^{j}\right)\right]+\frac{1}{2} \partial_{x^{\mu} x^{\nu}}^{2} \Phi\left(X_{t_{k}^{N}}\right) \beta^{\mu i} \beta^{\nu j}\left(B_{t_{k+1}^{N}}^{i}-B_{t_{k}^{N}}^{i}\right)\left(B_{t_{k+1}^{N}}^{j}-B_{t_{k}^{N}}^{j}\right)\right\}
\end{aligned}
$$

and

$$
\begin{aligned}
\zeta_{t}^{N}= & \frac{1}{2} \sum_{k=0}^{N-1} \partial_{x^{\mu} x^{\nu}}^{2} \Phi\left(X_{t_{k}^{N}}\right)\left\{\left[\alpha^{\mu}\left(t_{k+1}^{N}-t_{k}^{N}\right)+\eta^{\mu i j}\left(\left\langle B^{i}, B^{j}\right\rangle_{t_{k+1}^{N}}-\left\langle B^{i}, B^{j}\right\rangle_{t_{k}^{N}}\right)\right]\right. \\
& \times\left[\alpha^{\nu}\left(t_{k+1}^{N}-t_{k}^{N}\right)+\eta^{\nu l m}\left(\left\langle B^{l}, B^{m}\right\rangle_{t_{k+1}^{N}}-\left\langle B^{l}, B^{m}\right\rangle_{t_{k}^{N}}\right)\right] \\
& \left.+2\left[\alpha^{\mu}\left(t_{k+1}^{N}-t_{k}^{N}\right)+\eta^{\mu i j}\left(\left\langle B^{i}, B^{j}\right\rangle_{t_{k+1}^{N}}-\left\langle B^{i}, B^{j}\right\rangle_{t_{k}^{N}}\right)\right] \beta^{\nu l}\left(B_{t_{k+1}^{N}}^{l}-B_{t_{k}^{N}}^{l}\right)\right\} .
\end{aligned}
$$

We observe that, for each $u \in\left[t_{k}^{N}, t_{k+1}^{N}\right)$,

$$
\begin{aligned}
& \hat{\mathbb{E}}\left[\left|\partial_{x^{\nu}} \Phi\left(X_{u}\right)-\sum_{k=0}^{N-1} \partial_{x^{\nu}} \Phi\left(X_{t_{k}^{N}}\right) \mathbf{I}_{\left[t_{k}^{N}, t_{k+1}^{N}\right)}(u)\right|^{2}\right] \\
& =\hat{\mathbb{E}}\left[\left|\partial_{x^{\nu}} \Phi\left(X_{u}\right)-\partial_{x^{\nu}} \Phi\left(X_{t_{k}^{N}}\right)\right|^{2}\right] \\
& \leq c^{2} \hat{\mathbb{E}}\left[\left|X_{u}-X_{t_{k}^{N}}\right|^{2}\right] \leq C\left[\delta+\delta^{2}\right],
\end{aligned}
$$

where $c$ is the Lipschitz constant of $\left\{\partial_{x^{\nu}} \Phi\right\}_{\nu=1}^{n}$ and $C$ is a constant independent of $k$. Thus $\sum_{k=0}^{N-1} \partial_{x^{\nu}} \Phi\left(X_{t_{k}^{N}}\right) \mathbf{I}_{\left[t_{k}^{N}, t_{k+1}^{N}\right)}(\cdot)$ converges to $\partial_{x^{\nu}} \Phi\left(X\right.$.) in $M_{G}^{2}(0, T)$. Similarly, $\sum_{k=0}^{N-1} \partial_{x^{\mu} x^{\nu}}^{2} \Phi\left(X_{t_{k}^{N}}\right) \mathbf{I}_{\left[t_{k}^{N}, t_{k+1}^{N}\right)}(\cdot)$ converges to $\partial_{x^{\mu} x^{\nu}}^{2} \Phi\left(X\right.$.) in $M_{G}^{2}(0, T)$.

From Lemma 4.6 as well as the definitions of the integrations of $d t, d B_{t}$ and $d\langle B\rangle_{t}$, the limit of $\xi_{t}^{N}$ in $L_{G}^{2}\left(\Omega_{t}\right)$ is just the right hand side of (6.18). By the next Remark we also have $\zeta_{t}^{N} \rightarrow 0$ in $L_{G}^{2}\left(\Omega_{t}\right)$. We then have proved (6.18).

Remark 6.2 To prove $\zeta_{t}^{N} \rightarrow 0$ in $L_{G}^{2}\left(\Omega_{t}\right)$, we use the following estimates: for $\psi^{N} \in M_{G}^{2,0}(0, T)$ with $\psi_{t}^{N}=\sum_{k=0}^{N-1} \xi_{t_{k}}^{N} \mathbf{I}_{\left[t_{k}^{N}, t_{k+1}^{N}\right)}(t)$, and $\pi_{T}^{N}=\left\{t_{0}^{N}, \cdots, t_{N}^{N}\right\}$ such that $\lim _{N \rightarrow \infty} \mu\left(\pi_{T}^{N}\right)=0$ and $\hat{\mathbb{E}}\left[\sum_{k=0}^{N-1}\left|\xi_{t_{k}}^{N}\right|^{2}\left(t_{k+1}^{N}-t_{k}^{N}\right)\right] \leq C$, for all $N=$ $1,2, \cdots$, we have $\hat{\mathbb{E}}\left[\left|\sum_{k=0}^{N-1} \xi_{k}^{N}\left(t_{k+1}^{N}-t_{k}^{N}\right)^{2}\right|^{2}\right] \rightarrow 0$ and, for any fixed $\mathbf{a}, \overline{\mathbf{a}} \in \mathbb{R}^{d}$,

$$
\begin{aligned}
\hat{\mathbb{E}}\left[\left|\sum_{k=0}^{N-1} \xi_{k}^{N}\left(\left\langle B^{\mathbf{a}}\right\rangle_{t_{k+1}^{N}}-\left\langle B^{\mathbf{a}}\right\rangle_{t_{k}^{N}}\right)^{2}\right|^{2}\right] & \leq C \hat{\mathbb{E}}\left[\sum_{k=0}^{N-1}\left|\xi_{k}^{N}\right|^{2}\left(\left\langle B^{\mathbf{a}}\right\rangle_{t_{k+1}^{N}}-\left\langle B^{\mathbf{a}}\right\rangle_{t_{k}^{N}}\right)^{3}\right] \\
& \leq C \hat{\mathbb{E}}\left[\sum_{k=0}^{N-1}\left|\xi_{k}^{N}\right|^{2} \sigma_{\mathbf{a a}^{T}}^{6}\left(t_{k+1}^{N}-t_{k}^{N}\right)^{3}\right] \rightarrow 0,
\end{aligned}
$$




$$
\begin{aligned}
& \hat{\mathbb{E}}\left[\left|\sum_{k=0}^{N-1} \xi_{k}^{N}\left(\left\langle B^{\mathbf{a}}\right\rangle_{t_{k+1}^{N}}-\left\langle B^{\mathbf{a}}\right\rangle_{t_{k}^{N}}\right)\left(t_{k+1}^{N}-t_{k}^{N}\right)\right|^{2}\right] \\
\leq & C \hat{\mathbb{E}}\left[\sum_{k=0}^{N-1}\left|\xi_{k}^{N}\right|^{2}\left(t_{k+1}^{N}-t_{k}^{N}\right)\left(\left\langle B^{\mathbf{a}}\right\rangle_{t_{k+1}^{N}}-\left\langle B^{\mathbf{a}}\right\rangle_{t_{k}^{N}}\right)^{2}\right] \\
\leq & C \hat{\mathbb{E}}\left[\sum_{k=0}^{N-1}\left|\xi_{k}^{N}\right|^{2} \sigma_{\mathbf{a a}^{T}}^{4}\left(t_{k+1}^{N}-t_{k}^{N}\right)^{3}\right] \rightarrow 0,
\end{aligned}
$$

as well as

$$
\begin{aligned}
& \hat{\mathbb{E}}\left[\left|\sum_{k=0}^{N-1} \xi_{k}^{N}\left(t_{k+1}^{N}-t_{k}^{N}\right)\left(B_{t_{k+1}^{N}}^{\mathbf{a}}-B_{t_{k}^{N}}^{\mathbf{a}}\right)\right|^{2}\right] \\
\leq & C \hat{\mathbb{E}}\left[\sum_{k=0}^{N-1}\left|\xi_{k}^{N}\right|^{2}\left(t_{k+1}^{N}-t_{k}^{N}\right)\left|B_{t_{k+1}^{N}}^{\mathbf{a}}-B_{t_{k}^{N}}^{\mathbf{a}}\right|^{2}\right] \\
\leq & C \hat{\mathbb{E}}\left[\sum_{k=0}^{N-1}\left|\xi_{k}^{N}\right|^{2} \sigma_{\mathbf{a a}^{T}}^{2}\left(t_{k+1}^{N}-t_{k}^{N}\right)^{2}\right] \rightarrow 0
\end{aligned}
$$

and

$$
\begin{aligned}
& \hat{\mathbb{E}}\left[\left|\sum_{k=0}^{N-1} \xi_{k}^{N}\left(\left\langle B^{\mathbf{a}}\right\rangle_{t_{k+1}^{N}}-\left\langle B^{\mathbf{a}}\right\rangle_{t_{k}^{N}}\right)\left(B_{t_{k+1}^{N}}^{\overline{\mathbf{a}}}-B_{t_{k}^{N}}^{\overline{\bar{a}}}\right)\right|^{2}\right] \\
\leq & C \hat{\mathbb{E}}\left[\sum_{k=0}^{N-1}\left|\xi_{k}^{N}\right|^{2}\left(\left\langle B^{\mathbf{a}}\right\rangle_{t_{k+1}^{N}}-\left\langle B^{\mathbf{a}}\right\rangle_{t_{k}^{N}}\right) \mid B_{t_{k+1}^{N}}^{\overline{\mathbf{a}}}-B_{t_{k}^{N}}^{\left.\left.\overline{\overline{\mathbf{a}}}\right|^{2}\right]}\right. \\
\leq & C \hat{\mathbb{E}}\left[\sum_{k=0}^{N-1}\left|\xi_{k}^{N}\right|^{2} \sigma_{\mathbf{a a}^{T}}^{2} \sigma_{\overline{\mathbf{a}}^{\top} \overline{\mathbf{a}}^{T}}^{2}\left(t_{k+1}^{N}-t_{k}^{N}\right)^{2}\right] \rightarrow 0 .
\end{aligned}
$$

We now consider a general form of $G$-Itô's formula. Consider

$$
X_{t}^{\nu}=X_{0}^{\nu}+\int_{0}^{t} \alpha_{s}^{\nu} d s+\int_{0}^{t} \eta_{s}^{\nu i j} d\left\langle B^{i}, B^{j}\right\rangle_{s}+\int_{0}^{t} \beta_{s}^{\nu j} d B_{s}^{j}
$$

Proposition 6.3 Let $\Phi \in C^{2}\left(\mathbb{R}^{n}\right)$ with $\partial_{x^{\nu}} \Phi, \partial_{x^{\mu} x^{\nu}}^{2} \Phi \in C_{b . L i p}\left(\mathbb{R}^{n}\right)$ for $\mu, \nu=$ $1, \cdots, n$. Let $\alpha^{\nu}, \beta^{\nu j}$ and $\eta^{\nu i j}, \nu=1, \cdots, n, i, j=1, \cdots, d$ be bounded processes in $M_{G}^{2}(0, T)$. Then for each $t \geq 0$ we have, in $L_{G}^{2}\left(\Omega_{t}\right)$

$$
\begin{aligned}
\Phi\left(X_{t}\right)-\Phi\left(X_{s}\right)= & \int_{s}^{t} \partial_{x^{\nu}} \Phi\left(X_{u}\right) \beta_{u}^{\nu j} d B_{u}^{j}+\int_{s}^{t} \partial_{x^{\nu}} \Phi\left(X_{u}\right) \alpha_{u}^{\nu} d u \\
& +\int_{s}^{t}\left[\partial_{x^{\nu}} \Phi\left(X_{u}\right) \eta_{u}^{\nu i j}+\frac{1}{2} \partial_{x^{\mu} x^{\nu}}^{2} \Phi\left(X_{u}\right) \beta_{u}^{\mu i} \beta_{u}^{\nu j}\right] d\left\langle B^{i}, B^{j}\right\rangle_{u} .
\end{aligned}
$$


Proof. We first consider the case where $\alpha, \eta$ and $\beta$ are step processes of the form

$$
\eta_{t}(\omega)=\sum_{k=0}^{N-1} \xi_{k}(\omega) \mathbf{I}_{\left[t_{k}, t_{k+1}\right)}(t) .
$$

From the above lemma, it is clear that (6.20) holds true. Now let

$$
X_{t}^{\nu, N}=X_{0}^{\nu}+\int_{0}^{t} \alpha_{s}^{\nu, N} d s+\int_{0}^{t} \eta_{s}^{\nu i j, N} d\left\langle B^{i}, B^{j}\right\rangle_{s}+\int_{0}^{t} \beta_{s}^{\nu j, N} d B_{s}^{j},
$$

where $\alpha^{N}, \eta^{N}$ and $\beta^{N}$ are uniformly bounded step processes that converge to $\alpha, \eta$ and $\beta$ in $M_{G}^{2}(0, T)$ as $N \rightarrow \infty$, respectively. From Lemma 6.1.

$$
\begin{aligned}
\Phi\left(X_{t}^{N}\right)-\Phi\left(X_{s}^{N}\right)= & \int_{s}^{t} \partial_{x^{\nu}} \Phi\left(X_{u}^{N}\right) \beta_{u}^{\nu j, N} d B_{u}^{j}+\int_{s}^{t} \partial_{x^{\nu}} \Phi\left(X_{u}^{N}\right) \alpha_{u}^{\nu, N} d u \\
& +\int_{s}^{t}\left[\partial_{x^{\nu}} \Phi\left(X_{u}^{N}\right) \eta_{u}^{\nu i j, N}+\frac{1}{2} \partial_{x^{\mu} x^{\nu}}^{2} \Phi\left(X_{u}^{N}\right) \beta_{u}^{\mu i, N} \beta_{u}^{\nu j, N}\right] d\left\langle B^{i}, B^{j}\right\rangle_{u} .
\end{aligned}
$$

Since

$$
\begin{aligned}
& \hat{\mathbb{E}}\left[\left|X_{t}^{\nu, N}-X_{t}^{\nu}\right|^{2}\right] \\
\leq & C \hat{\mathbb{E}}\left[\int_{0}^{T}\left[\left(\alpha_{s}^{\nu, N}-\alpha_{s}^{\nu}\right)^{2}+\left|\eta_{s}^{\nu, N}-\eta_{s}^{\nu}\right|^{2}+\left|\beta_{s}^{\nu, N}-\beta_{s}^{\nu}\right|^{2}\right] d s\right],
\end{aligned}
$$

where $C$ is a constant independent of $N$, we can prove that, in $M_{G}^{2}(0, T)$,

$$
\begin{aligned}
\partial_{x^{\nu}} \Phi\left(X^{N}\right) \eta^{\nu i j, N} & \rightarrow \partial_{x^{\nu}} \Phi(X .) \eta^{\nu i j} \\
\partial_{x^{\mu} x^{\nu}}^{2} \Phi\left(X_{\cdot}^{N}\right) \beta^{\mu i, N} \beta^{\nu j, N} & \rightarrow \partial_{x^{\mu} x^{\nu}}^{2} \Phi(X .) \beta^{\mu i} \beta_{\cdot}^{\nu j}, \\
\partial_{x^{\nu}} \Phi\left(X^{N}\right) \alpha^{\nu, N} & \rightarrow \partial_{x^{\nu}} \Phi(X .) \alpha_{.}^{\nu}, \\
\partial_{x^{\nu}} \Phi\left(X^{N}\right) \beta^{\nu j, N} & \rightarrow \partial_{x^{\nu}} \Phi(X .) \beta_{\cdot}^{\nu j} .
\end{aligned}
$$

We then can pass to limit as $N \rightarrow \infty$ in both sides of (6.21) to get (6.20).

In order to consider the general $\Phi$, we first prove a useful inequality.

For the $G$-expectation $\hat{\mathbb{E}}$, we have the following representation (see Chap VI):

$$
\hat{\mathbb{E}}[X]=\sup _{P \in \mathcal{P}} E_{P}[X] \text { for } X \in L_{G}^{1}(\Omega),
$$

where $\mathcal{P}$ is a weakly compact family of probability measures on $(\Omega, \mathcal{B}(\Omega))$.

Proposition 6.4 Let $\beta \in M_{G}^{p}(0, T)$ with $p \geq 2$ and let $\mathbf{a} \in \mathbb{R}^{d}$ be fixed. Then we have $\int_{0}^{T} \beta_{t} d B_{t}^{\mathbf{a}} \in L_{G}^{p}\left(\Omega_{T}\right)$ and

$$
\hat{\mathbb{E}}\left[\left|\int_{0}^{T} \beta_{t} d B_{t}^{\mathbf{a}}\right|^{p}\right] \leq C_{p} \hat{\mathbb{E}}\left[\left|\int_{0}^{T} \beta_{t}^{2} d\left\langle B^{\mathbf{a}}\right\rangle_{t}\right|^{p / 2}\right] .
$$


Proof. It suffices to consider the case where $\beta$ is a step process of the form

$$
\beta_{t}(\omega)=\sum_{k=0}^{N-1} \xi_{k}(\omega) \mathbf{I}_{\left[t_{k}, t_{k+1}\right)}(t) .
$$

For each $\xi \in L_{i p}\left(\Omega_{t}\right)$ with $t \in[0, T]$, we have

$$
\hat{\mathbb{E}}\left[\xi \int_{t}^{T} \beta_{s} d B_{s}^{\mathbf{a}}\right]=0 .
$$

From this we can easily get $E_{P}\left[\xi \int_{t}^{T} \beta_{s} d B_{s}^{\mathbf{a}}\right]=0$ for each $P \in \mathcal{P}$, which implies that $\left(\int_{0}^{t} \beta_{s} d B_{s}^{\mathbf{a}}\right)_{t \in 0, T]}$ is a $P$-martingale. Similarly we can prove that

$$
M_{t}:=\left(\int_{0}^{t} \beta_{s} d B_{s}^{\mathbf{a}}\right)^{2}-\int_{0}^{t} \beta_{s}^{2} d\left\langle B^{\mathbf{a}}\right\rangle_{s}, \quad t \in[0, T]
$$

is a $P$-martingale for each $P \in \mathcal{P}$. By the Burkholder-Davis-Gundy inequalities, we have

$$
E_{P}\left[\left|\int_{0}^{T} \beta_{t} d B_{t}^{\mathbf{a}}\right|^{p}\right] \leq C_{p} E_{P}\left[\left|\int_{0}^{T} \beta_{t}^{2} d\left\langle B^{\mathbf{a}}\right\rangle_{t}\right|^{p / 2}\right] \leq C_{p} \hat{\mathbb{E}}\left[\left|\int_{0}^{T} \beta_{t}^{2} d\left\langle B^{\mathbf{a}}\right\rangle_{t}\right|^{p / 2}\right],
$$

where $C_{p}$ is a universal constant independent of $P$. Thus we get (6.23).

We now give the general $G$-Itô's formula.

Theorem 6.5 Let $\Phi$ be a $C^{2}$-function on $\mathbb{R}^{n}$ such that $\partial_{x^{\mu} x^{\nu}}^{2} \Phi$ satisfy polynomial growth condition for $\mu, \nu=1, \cdots, n$. Let $\alpha^{\nu}, \beta^{\nu j}$ and $\eta^{\nu i j}, \nu=1, \cdots, n$, $i, j=1, \cdots, d$ be bounded processes in $M_{G}^{2}(0, T)$. Then for each $t \geq 0$ we have in $L_{G}^{2}\left(\Omega_{t}\right)$

$$
\begin{aligned}
\Phi\left(X_{t}\right)-\Phi\left(X_{s}\right)= & \int_{s}^{t} \partial_{x^{\nu}} \Phi\left(X_{u}\right) \beta_{u}^{\nu j} d B_{u}^{j}+\int_{s}^{t} \partial_{x^{\nu}} \Phi\left(X_{u}\right) \alpha_{u}^{\nu} d u \\
& +\int_{s}^{t}\left[\partial_{x^{\nu}} \Phi\left(X_{u}\right) \eta_{u}^{\nu i j}+\frac{1}{2} \partial_{x^{\mu} x^{\nu}}^{2} \Phi\left(X_{u}\right) \beta_{u}^{\mu i} \beta_{u}^{\nu j}\right] d\left\langle B^{i}, B^{j}\right\rangle_{u} .
\end{aligned}
$$

Proof. By the assumptions on $\Phi$, we can choose a sequence of functions $\Phi_{N} \in$ $C_{0}^{2}\left(\mathbb{R}^{n}\right)$ such that

$\left|\Phi_{N}(x)-\Phi(x)\right|+\left|\partial_{x^{\nu}} \Phi_{N}(x)-\partial_{x^{\nu}} \Phi(x)\right|+\left|\partial_{x^{\mu} x^{\nu}}^{2} \Phi_{N}(x)-\partial_{x^{\mu} x^{\nu}}^{2} \Phi(x)\right| \leq \frac{C_{1}}{N}\left(1+|x|^{k}\right)$,

where $C_{1}$ and $k$ are positive constants independent of $N$. Obviously, $\Phi_{N}$ satisfies the conditions in Proposition 6.3, therefore,

$$
\begin{aligned}
\Phi_{N}\left(X_{t}\right)-\Phi_{N}\left(X_{s}\right)= & \int_{s}^{t} \partial_{x^{\nu}} \Phi_{N}\left(X_{u}\right) \beta_{u}^{\nu j} d B_{u}^{j}+\int_{s}^{t} \partial_{x^{v}} \Phi_{N}\left(X_{u}\right) \alpha_{u}^{\nu} d u \\
& +\int_{s}^{t}\left[\partial_{x^{\nu}} \Phi_{N}\left(X_{u}\right) \eta_{u}^{\nu i j}+\frac{1}{2} \partial_{x^{\mu} x^{\nu}}^{2} \Phi_{N}\left(X_{u}\right) \beta_{u}^{\mu i} \beta_{u}^{\nu j}\right] d\left\langle B^{i}, B^{j}\right\rangle_{u} .
\end{aligned}
$$


For each fixed $T>0$, by Proposition 6.4 there exists a constant $C_{2}$ such that

$$
\hat{\mathbb{E}}\left[\left|X_{t}\right|^{2 k}\right] \leq C_{2} \text { for } t \in[0, T] .
$$

Thus we can prove that $\Phi_{N}\left(X_{t}\right) \rightarrow \Phi\left(X_{t}\right)$ in $L_{G}^{2}\left(\Omega_{t}\right)$ and in $M_{G}^{2}(0, T)$,

$$
\begin{aligned}
\partial_{x^{\nu}} \Phi_{N}(X .) \eta^{\nu i j} & \rightarrow \partial_{x^{\nu}} \Phi(X .) \eta_{.}^{\nu i j}, \\
\partial_{x^{\mu} x^{\nu}}^{2} \Phi_{N}(X .) \beta_{\cdot}^{\mu i} \beta^{\nu j} & \rightarrow \partial_{x^{\mu} x^{\nu}}^{2} \Phi(X .) \beta_{\cdot}^{\mu i} \beta_{\cdot}^{\nu j}, \\
\partial_{x^{\nu}} \Phi_{N}(X .) \alpha_{.}^{\nu} & \rightarrow \partial_{x^{\nu}} \Phi(X .) \alpha_{.}^{\nu}, \\
\partial_{x^{\nu}} \Phi_{N}(X .) \beta^{\nu j} & \rightarrow \partial_{x^{\nu}} \Phi(X .) \beta^{\nu j} .
\end{aligned}
$$

We then can pass to limit as $N \rightarrow \infty$ in both sides of (6.25) to get (6.24).

Corollary 6.6 Let $\Phi$ be a polynomial and $\mathbf{a}, \mathbf{a}^{\nu} \in \mathbb{R}^{d}$ be fixed for $\nu=1, \cdots, n$. Then we have

$$
\Phi\left(X_{t}\right)-\Phi\left(X_{s}\right)=\int_{s}^{t} \partial_{x^{\nu}} \Phi\left(X_{u}\right) d B_{u}^{\mathbf{a}^{\nu}}+\frac{1}{2} \int_{s}^{t} \partial_{x^{\mu} x^{\nu}}^{2} \Phi\left(X_{u}\right) d\left\langle B^{\mathbf{a}^{\mu}}, B^{\mathbf{a}^{\nu}}\right\rangle_{u},
$$

where $X_{t}=\left(B_{t}^{\mathbf{a}^{1}}, \cdots, B_{t}^{\mathbf{a}^{n}}\right)^{T}$. In particular, we have, for $k=2,3, \cdots$,

$$
\left(B_{t}^{\mathbf{a}}\right)^{k}=k \int_{0}^{t}\left(B_{s}^{\mathbf{a}}\right)^{k-1} d B_{s}^{\mathbf{a}}+\frac{k(k-1)}{2} \int_{0}^{t}\left(B_{s}^{\mathbf{a}}\right)^{k-2} d\left\langle B^{\mathbf{a}}\right\rangle_{s} .
$$

If $\hat{\mathbb{E}}$ becomes a linear expectation, then the above $G$-Itô's formula is the classical one.

\section{$\S 7$ Generalized G-Brownian Motion}

Let $G: \mathbb{R}^{d} \times \mathbb{S}(d) \rightarrow \mathbb{R}$ be a given continuous sublinear function monotonic in $A \in \mathbb{S}(d)$. Then by Theorem 2.1 in Chap, [- there exists a bounded, convex and closed subset $\Sigma \subset \mathbb{R}^{d} \times \mathbb{S}_{+}(d)$ such that

$$
G(p, A)=\sup _{(q, B) \in \Sigma}\left[\frac{1}{2} \operatorname{tr}[A B]+\langle p, q\rangle\right] \quad \text { for }(p, A) \in \mathbb{R}^{d} \times \mathbb{S}(d) .
$$

By Chapter $[$ we know that there exists a pair of $d$-dimensional random vectors $(X, Y)$ which is $G$-distributed.

We now give the definition of the generalized $G$-Brownian motion.

Definition $7.1 A$ d-dimensional process $\left(B_{t}\right)_{t \geq 0}$ on a sublinear expectation space $(\Omega, \mathcal{H}, \hat{\mathbb{E}})$ is called a generalized $G$-Brownian motion if the following properties are satisfied:

(i) $B_{0}(\omega)=0$;

(ii) For each $t, s \geq 0$, the increment $B_{t+s}-B_{t}$ identically distributed with $\sqrt{s} X+s Y$ and is independent from $\left(B_{t_{1}}, B_{t_{2}}, \cdots, B_{t_{n}}\right)$, for each $n \in \mathbb{N}$ and $0 \leq t_{1} \leq \cdots \leq t_{n} \leq t$, where $(X, Y)$ is $G$-distributed. 
The following theorem gives a characterization of the generalized $G$-Brownian motion.

Theorem 7.2 Let $\left(B_{t}\right)_{t \geq 0}$ be a d-dimensional process defined on a sublinear expectation space $(\Omega, \mathcal{H}, \hat{\mathbb{E}})$ such that

(i) $B_{0}(\omega)=0$;

(ii) For each $t, s \geq 0, B_{t+s}-B_{t}$ and $B_{s}$ are identically distributed and $B_{t+s}-B_{t}$ is independent from $\left(B_{t_{1}}, B_{t_{2}}, \cdots, B_{t_{n}}\right)$, for each $n \in \mathbb{N}$ and $0 \leq t_{1} \leq \cdots \leq t_{n} \leq$ $t$.

(iii) $\lim _{t \downarrow 0} \hat{\mathbb{E}}\left[\left|B_{t}\right|^{3}\right] t^{-1}=0$.

Then $\left(B_{t}\right)_{t \geq 0}$ is a generalized $G$-Brownian motion with $G(p, A)=\lim _{\delta \downarrow 0} \hat{\mathbb{E}}\left[\left\langle p, B_{\delta}\right\rangle+\right.$ $\left.\frac{1}{2}\left\langle A B_{\delta}, B_{\delta}\right\rangle\right] \delta^{-1} \quad$ for $(p, A) \in \mathbb{R}^{d} \times \mathbb{S}(d)$.

Proof. We first prove that $\lim _{\delta \downarrow 0} \hat{\mathbb{E}}\left[\left\langle p, B_{\delta}\right\rangle+\frac{1}{2}\left\langle A B_{\delta}, B_{\delta}\right\rangle\right] \delta^{-1}$ exists. For each fixed $(p, A) \in \mathbb{R}^{d} \times \mathbb{S}(d)$, we set

$$
f(t):=\hat{\mathbb{E}}\left[\left\langle p, B_{t}\right\rangle+\frac{1}{2}\left\langle A B_{t}, B_{t}\right\rangle\right]
$$

Since

$$
|f(t+h)-f(t)| \leq \hat{\mathbb{E}}\left[\left(|p|+2|A|\left|B_{t}\right|\right)\left|B_{t+h}-B_{t}\right|+|A|\left|B_{t+h}-B_{t}\right|^{2}\right] \rightarrow 0
$$

we get that $f(t)$ is a continuous function. It is easy to prove that

$$
\hat{\mathbb{E}}\left[\left\langle q, B_{t}\right\rangle\right]=\hat{\mathbb{E}}\left[\left\langle q, B_{1}\right\rangle\right] t \text { for } q \in \mathbb{R}^{d}
$$

Thus for each $t, s>0$,

$$
|f(t+s)-f(t)-f(s)| \leq C \hat{\mathbb{E}}\left[\left|B_{t}\right|\right] s
$$

where $C=|A| \hat{\mathbb{E}}\left[\left|B_{1}\right|\right]$. By (iii), there exists a constant $\delta_{0}>0$ such that $\hat{\mathbb{E}}\left[\left|B_{t}\right|^{3}\right] \leq t$ for $t \leq \delta_{0}$. Thus for each fixed $t>0$ and $N \in \mathbb{N}$ such that $N t \leq \delta_{0}$, we have

$$
|f(N t)-N f(t)| \leq \frac{3}{4} C(N t)^{4 / 3}
$$

From this and the continuity of $f$, it is easy to show that $\lim _{t \downarrow 0} f(t) t^{-1}$ exists. Thus we can get $G(p, A)$ for each $(p, A) \in \mathbb{R}^{d} \times \mathbb{S}(d)$. It is also easy to check that $G$ is a continuous sublinear function monotonic in $A \in \mathbb{S}(d)$.

We only need to prove that, for each fixed $\varphi \in C_{b . L i p}\left(\mathbb{R}^{d}\right)$, the function

$$
u(t, x):=\hat{\mathbb{E}}\left[\varphi\left(x+B_{t}\right)\right], \quad(t, x) \in[0, \infty) \times \mathbb{R}^{d}
$$

is the viscosity solution of the following parabolic PDE:

$$
\partial_{t} u-G\left(D u, D^{2} u\right)=0,\left.\quad u\right|_{t=0}=\varphi
$$


We first prove that $u$ is Lipschitz in $x$ and $\frac{1}{2}$-Hölder continuous in $t$. In fact, for each fixed $t, u(t, \cdot) \in C_{b . L i p}\left(\mathbb{R}^{d}\right)$ since

$$
\begin{aligned}
\left|\hat{\mathbb{E}}\left[\varphi\left(x+B_{t}\right)\right]-\hat{\mathbb{E}}\left[\varphi\left(y+B_{t}\right)\right]\right| & \leq \hat{\mathbb{E}}\left[\left|\varphi\left(x+B_{t}\right)-\varphi\left(y+B_{t}\right)\right|\right] \\
& \leq C|x-y| .
\end{aligned}
$$

For each $\delta \in[0, t]$, since $B_{t}-B_{\delta}$ is independent from $B_{\delta}$,

$$
\begin{aligned}
u(t, x) & =\hat{\mathbb{E}}\left[\varphi\left(x+B_{\delta}+\left(B_{t}-B_{\delta}\right)\right]\right. \\
& =\hat{\mathbb{E}}\left[\hat{\mathbb{E}}\left[\varphi\left(y+\left(B_{t}-B_{\delta}\right)\right)\right]_{y=x+B_{\delta}} .\right.
\end{aligned}
$$

Hence

$$
u(t, x)=\hat{\mathbb{E}}\left[u\left(t-\delta, x+B_{\delta}\right)\right]
$$

Thus

$$
\begin{aligned}
|u(t, x)-u(t-\delta, x)| & =\left|\hat{\mathbb{E}}\left[u\left(t-\delta, x+B_{\delta}\right)-u(t-\delta, x)\right]\right| \\
& \leq \hat{\mathbb{E}}\left[\left|u\left(t-\delta, x+B_{\delta}\right)-u(t-\delta, x)\right|\right] \\
& \leq \hat{\mathbb{E}}\left[C\left|B_{\delta}\right|\right] \leq C \sqrt{G(0, I)+1} \sqrt{\delta} .
\end{aligned}
$$

To prove that $u$ is a viscosity solution of (7.26), we fix a $(t, x) \in(0, \infty) \times \mathbb{R}^{d}$ and let $v \in C_{b}^{2,3}\left([0, \infty) \times \mathbb{R}^{d}\right)$ be such that $v \geq u$ and $v(t, x)=u(t, x)$. From (7.27), we have

$$
v(t, x)=\hat{\mathbb{E}}\left[u\left(t-\delta, x+B_{\delta}\right)\right] \leq \hat{\mathbb{E}}\left[v\left(t-\delta, x+B_{\delta}\right)\right] .
$$

Therefore, by Taylor's expansion,

$$
\begin{aligned}
0 & \leq \hat{\mathbb{E}}\left[v\left(t-\delta, x+B_{\delta}\right)-v(t, x)\right] \\
& =\hat{\mathbb{E}}\left[v\left(t-\delta, x+B_{\delta}\right)-v\left(t, x+B_{\delta}\right)+\left(v\left(t, x+B_{\delta}\right)-v(t, x)\right)\right] \\
& =\hat{\mathbb{E}}\left[-\partial_{t} v(t, x) \delta+\left\langle D v(t, x), B_{\delta}\right\rangle+\frac{1}{2}\left\langle D^{2} v(t, x) B_{\delta}, B_{\delta}\right\rangle+I_{\delta}\right] \\
& \leq-\partial_{t} v(t, x) \delta+\hat{\mathbb{E}}\left[\left\langle D v(t, x), B_{\delta}\right\rangle+\frac{1}{2}\left\langle D^{2} v(t, x) B_{\delta}, B_{\delta}\right\rangle\right]+\hat{\mathbb{E}}\left[I_{\delta}\right],
\end{aligned}
$$

where

$$
\begin{aligned}
I_{\delta}= & \int_{0}^{1}-\left[\partial_{t} v\left(t-\beta \delta, x+B_{\delta}\right)-\partial_{t} v(t, x)\right] \delta d \beta \\
& +\int_{0}^{1} \int_{0}^{1}\left\langle\left(D^{2} v\left(t, x+\alpha \beta B_{\delta}\right)-D^{2} v(t, x)\right) B_{\delta}, B_{\delta}\right\rangle \alpha d \beta d \alpha .
\end{aligned}
$$

With the assumption (iii) we can check that $\lim _{\delta \downarrow 0} \hat{\mathbb{E}}\left[\left|I_{\delta}\right|\right] \delta^{-1}=0$, from which we get $\partial_{t} v(t, x)-G\left(D v(t, x), D^{2} v(t, x)\right) \leq 0$, hence $u$ is a viscosity subsolution of (7.26). We can analogously prove that $u$ is a viscosity supersolution. Thus $u$ is a viscosity solution and $\left(B_{t}\right)_{t \geq 0}$ is a generalized $G$-Brownian motion. 
In many situations we are interested in a generalized $2 d$-dimensional Brownian motion $\left(B_{t}, b_{t}\right)_{t \geq 0}$ such that $\hat{\mathbb{E}}\left[B_{t}\right]=-\hat{\mathbb{E}}\left[-B_{t}\right]=0$ and $\hat{\mathbb{E}}\left[\left|b_{t}\right|^{2}\right] / t \rightarrow 0$, as $t \downarrow 0$. In this case $B$ is in fact a $G$-Brownian motion defined on Definition 2.1 of Chapter II Moreover the process $b$ satisfies properties of Proposition 5.2. We define $u(t, x, y)=\hat{\mathbb{E}}\left[\varphi\left(x+B_{t}, y+b_{t}\right)\right]$. By the above proposition it follows that $u$ is the solution of the PDE

$$
\partial_{t} u=G\left(D_{y} u, D_{x x}^{2} u\right),\left.\quad u\right|_{t=0}=\varphi \in C_{l . L i p}\left(\mathbb{R}^{2 d}\right) .
$$

where $G$ is a sublinear function of $(p, A) \in \mathbb{R}^{d}$, defined by

$$
G(p, A):=\hat{\mathbb{E}}\left[\left\langle p, b_{t}\right\rangle+\left\langle A B_{t}, B_{t}\right\rangle\right] .
$$

Here $\langle\cdot, \cdot\rangle=\langle\cdot, \cdot\rangle_{\mathbb{R}^{d}} \cdot$

\section{$\S 8 \widetilde{G}$-Brownian Motion under a Nonlinear Ex- pectation}

We can also define a $G$-Brownian motion on a nonlinear expectation space $(\Omega, \mathcal{H}, \widetilde{\mathbb{E}})$.

Definition 8.1 A d-dimensional process $\left(B_{t}\right)_{t \geq 0}$ on a nonlinear expectation space $(\Omega, \mathcal{H}, \widetilde{\mathbb{E}})$ is called a (nonlinear) $\widetilde{G}$-Brownian motion if the following properties are satisfied:

(i) $B_{0}(\omega)=0$;

(ii) For each $t, s \geq 0$, the increment $B_{t+s}-B_{t}$ identically distributed with $B_{s}$ and is independent from $\left(B_{t_{1}}, B_{t_{2}}, \cdots, B_{t_{n}}\right)$, for each $n \in \mathbb{N}$ and $0 \leq t_{1} \leq \cdots \leq$ $t_{n} \leq t$

(iii) $\lim _{t \downarrow 0} \hat{\mathbb{E}}\left[\left|B_{t}\right|^{3}\right] t^{-1}=0$.

The following theorem gives a characterization of the nonlinear $\widetilde{G}$-Brownian motion, and give us the generator $\widetilde{G}$ of our $\widetilde{G}$-Brownian motion.

Theorem 8.2 Let $\underset{\mathbb{E}}{\widetilde{C}}$ be a nonlinear expectation and $\hat{\mathbb{E}}$ be a sublinear expectation defined on $(\Omega, \mathcal{H})$. let $\widetilde{\mathbb{E}}$ be dominated by $\hat{\mathbb{E}}$, namely

$$
\widetilde{\mathbb{E}}[X]-\widetilde{\mathbb{E}}[Y] \leq \hat{\mathbb{E}}[X-Y], \quad X, Y \in \mathcal{H}
$$

Let $\left(B_{t}, b_{t}\right)_{t \geq 0}$ be a given $\mathbb{R}^{2 d}$-valued $\widetilde{G}$-Brownian motion on $(\Omega, \mathcal{H}, \widetilde{\mathbb{E}})$ such that $\hat{\mathbb{E}}\left[B_{t}\right]=\hat{\mathbb{E}}\left[-B_{t}\right]=0$ and $\lim _{t \rightarrow 0} \hat{\mathbb{E}}\left[\left|b_{t}\right|^{2}\right] / t=0$. Then, for each fixed $\varphi \in$ $C_{\text {b.Lip }}\left(\mathbb{R}^{2 d}\right)$, the function

$$
\tilde{u}(t, x, y):=\widetilde{\mathbb{E}}\left[\varphi\left(x+B_{t}, y+b_{t}\right)\right], \quad(t, x, y) \in[0, \infty) \times \mathbb{R}^{2 d}
$$

is the viscosity solution of the following parabolic PDE:

$$
\partial_{t} \tilde{u}-\widetilde{G}\left(D_{y} \tilde{u}, D_{x}^{2} \tilde{u}\right)=0,\left.\quad u\right|_{t=0}=\varphi .
$$


where

$$
\widetilde{G}(p, A)=\widetilde{\mathbb{E}}\left[\left\langle p, b_{1}\right\rangle+\frac{1}{2}\left\langle A B_{1}, B_{1}\right\rangle\right],(p, A) \in \mathbb{R}^{d} \times \mathbb{S}(d)
$$

Remark 8.3 Let $G(p, A):=\hat{\mathbb{E}}\left[\left\langle p, b_{1}\right\rangle+\frac{1}{2}\left\langle A B_{1}, B_{1}\right\rangle\right]$. Then the function $\widetilde{G}$ is dominated by the sublinear function $G$ in the following sense:

$$
\widetilde{G}(p, A)-\widetilde{G}\left(p^{\prime}, A^{\prime}\right) \leq G\left(p-p^{\prime}, A-A^{\prime}\right), \quad(p, A), \quad\left(p^{\prime}, A^{\prime}\right) \in \mathbb{R}^{d} \times \mathbb{S}(d) .
$$

Proof of Theorem 8.2, We set

$$
f(t)=f_{A, t}(t):=\widetilde{\mathbb{E}}\left[\left\langle p, b_{t}\right\rangle+\frac{1}{2}\left\langle A B_{t}, B_{t}\right\rangle\right], t \geq 0 .
$$

Since

$$
|f(t+h)-f(t)| \leq \hat{\mathbb{E}}\left[\left(|p|+2|A|\left|B_{t}\right|\right)\left|B_{t+h}-B_{t}\right|+|A|\left|B_{t+h}-B_{t}\right|^{2}\right] \rightarrow 0,
$$

we get that $f(t)$ is a continuous function. Since $\hat{\mathbb{E}}\left[B_{t}\right]=\hat{\mathbb{E}}\left[-B_{t}\right]=0$, it follows from Proposition 3.7 that $\widetilde{\mathbb{E}}\left[X+\left\langle p, B_{t}\right\rangle\right]=\widetilde{\mathbb{E}}[X]$ for each $X \in \mathcal{H}$ and $p \in \mathbb{R}^{d}$. Thus

$$
\begin{aligned}
f(t+h)= & \widetilde{\mathbb{E}}\left[\left\langle p, b_{t+h}-b_{t}\right\rangle+\left\langle p, b_{t}\right\rangle\right. \\
& \left.+\frac{1}{2}\left\langle A B_{t+h}-B_{t}, B_{t+h}-B_{t}\right\rangle+\frac{1}{2}\left\langle A B_{t}, B_{t}\right\rangle\right] \\
= & \widetilde{\mathbb{E}}\left[\left\langle p, b_{h}\right\rangle+\frac{1}{2}\left\langle A B_{h}, B_{h}\right\rangle\right]+\widetilde{\mathbb{E}}\left[\left\langle p, b_{t}\right\rangle+\frac{1}{2}\left\langle A B_{t}, B_{t}\right\rangle\right] \\
= & f(t)+f(h) .
\end{aligned}
$$

It then follows that $f(t)=f(1) t=\widetilde{G}(A, p) t$. We now prove that the function $u$ is Lipschitz in $x$ and uniformly continuous in $t$. In fact, for each fixed $t$, $u(t, \cdot) \in C_{b . L i p}\left(\mathbb{R}^{d}\right)$ since

$$
\begin{aligned}
& \left|\widetilde{\mathbb{E}}\left[\varphi\left(x+B_{t}, y+b_{t}\right)\right]-\widetilde{\mathbb{E}}\left[\varphi\left(x^{\prime}+B_{t}, y^{\prime}+b_{t}\right)\right]\right| \\
& \leq \hat{\mathbb{E}}\left[\left|\varphi\left(x+B_{t}, y+b_{t}\right)-\varphi\left(x^{\prime}+B_{t}, y^{\prime}+b_{t}\right)\right|\right] \leq C\left(\left|x-x^{\prime}\right|+\left|y-y^{\prime}\right|\right) .
\end{aligned}
$$

For each $\delta \in[0, t]$, since $\left(B_{t}-B_{\delta}, b_{t}-b_{\delta}\right)$ is independent from $\left(B_{\delta}, b_{\delta}\right)$,

$$
\begin{aligned}
\tilde{u}(t, x) & =\widetilde{\mathbb{E}}\left[\varphi\left(x+B_{\delta}+\left(B_{t}-B_{\delta}\right), y+b_{\delta}+\left(b_{t}-b_{\delta}\right)\right]\right. \\
& =\widetilde{\mathbb{E}}\left[\widetilde{\mathbb{E}}\left[\varphi\left(\bar{x}+\left(B_{t}-B_{\delta}\right), \bar{y}+\left(b_{t}-b_{\delta}\right)\right)\right]_{\bar{x}=x+B_{\delta}, \bar{y}=y+b_{\delta}}\right] .
\end{aligned}
$$

Hence

$$
u(t, x)=\hat{\mathbb{E}}\left[u\left(t-\delta, x+B_{\delta}, y+b_{\delta}\right)\right] .
$$

Thus

$$
\begin{aligned}
|\tilde{u}(t, x, y)-\tilde{u}(t-\delta, x, y)| & =\left|\tilde{\mathbb{E}}\left[\tilde{u}\left(t-\delta, x+B_{\delta}, y+b_{\delta}\right)-\tilde{u}(t-\delta, x, y)\right]\right| \\
& \leq \hat{\mathbb{E}}\left[\tilde{u}\left(t-\delta, x+B_{\delta}, y+b_{\delta}\right)-\tilde{u}(t-\delta, x, y) \mid\right] \\
& \leq \hat{\mathbb{E}}\left[C\left(\left|B_{\delta}\right|+\left|b_{\delta}\right|\right)\right] .
\end{aligned}
$$


It follows from (iii) of Definition 8.1 that $u(t, x, y)$ is continuous in $t$ uniformly in $(t, x) \in[0, \infty) \times \mathbb{R}^{2 d}$.

To prove that $\tilde{u}$ is a viscosity solution of (8.28), we fix a $(t, x, y) \in(0, \infty) \times \mathbb{R}^{2 d}$ and let $v \in C_{b}^{2,3}\left([0, \infty) \times \mathbb{R}^{2 d}\right)$ be such that $v \geq u$ and $v(t, x, y)=u(t, x, y)$. From (8.30), we have

$$
v(t, x, y)=\widetilde{\mathbb{E}}\left[u\left(t-\delta, x+B_{\delta}, y+b_{\delta}\right)\right] \leq \widetilde{\mathbb{E}}\left[v\left(t-\delta, x+B_{\delta}, y+b_{\delta}\right)\right] .
$$

Therefore, by Taylor's expansion,

$$
\begin{aligned}
0 \leq \widetilde{\mathbb{E}} & \left.v\left(t-\delta, x+B_{\delta}, y+b_{\delta}\right)-v(t, x)\right] \\
= & \widetilde{\mathbb{E}}\left[v\left(t-\delta, x+B_{\delta}, y+b_{\delta}\right)-v\left(t, x+B_{\delta}, y+b_{\delta}\right)\right. \\
& +\left(v\left(t, x+B_{\delta}, y+b_{\delta}\right)-v(t, x, y)\right] \\
= & \widetilde{\mathbb{E}}\left[-\partial_{t} v(t, x, y) \delta+\left\langle D_{y} v(t, x, y), b_{\delta}\right\rangle+\left\langle\partial_{x} v(t, x, y), B_{\delta}\right\rangle+\frac{1}{2}\left\langle D_{x x}^{2} v(t, x, y) B_{\delta}, B_{\delta}\right\rangle+I_{\delta}\right] \\
\leq & -\partial_{t} v(t, x, y) \delta+\widetilde{\mathbb{E}}\left[\left\langle D_{y} v(t, x, y), b_{\delta}\right\rangle+\frac{1}{2}\left\langle D_{x x}^{2} v(t, x, y) B_{\delta}, B_{\delta}\right\rangle\right]+\hat{\mathbb{E}}\left[I_{\delta}\right]
\end{aligned}
$$

where

$$
\begin{aligned}
I_{\delta} & =\int_{0}^{1}-\left[\partial_{t} v\left(t-\delta \gamma, x+B_{\delta}, y+b_{\delta}\right)-\partial_{t} v(t, x, y)\right] \delta d \gamma \\
& +\int_{0}^{1}\left\langle\partial_{y} v\left(t, x+\gamma B_{\delta}, y+\gamma b_{\delta}\right)-\partial_{y} v(t, x, y), b_{\delta}\right\rangle d \gamma \\
& +\int_{0}^{1}\left\langle\partial_{x} v\left(t, x, y+\gamma b_{\delta}\right)-\partial_{x} v(t, x, y), B_{\delta}\right\rangle d \gamma \\
& +\int_{0}^{1} \int_{0}^{1}\left\langle\left(D_{x x}^{2} v\left(t, x+\alpha \gamma B_{\delta}, y+\gamma b_{\delta}\right)-D_{x x}^{2} v(t, x, y)\right) B_{\delta}, B_{\delta}\right\rangle \gamma d \gamma d \alpha .
\end{aligned}
$$

With the assumption (iii) we can check that $\lim _{\delta \downarrow 0} \hat{\mathbb{E}}\left[\left|I_{\delta}\right|\right] \delta^{-1}=0$, from which we get $\partial_{t} v(t, x)-G\left(D v(t, x), D^{2} v(t, x)\right) \leq 0$, hence $u$ is a viscosity subsolution of (8.28). We can analogously prove that $u$ is a viscosity supersolution. Thus $u$ is a viscosity solution.

\section{$\S 9$ Construction of $\widetilde{G}$-Brownian Motions under Nonlinear Expectation}

Let $G(\cdot): \mathbb{R}^{d} \times \mathbb{S}(d) \rightarrow \mathbb{R}$ be a given sublinear function monotonic on $A \in \mathbb{S}(d)$ and $\widetilde{G}(\cdot): \mathbb{R}^{d} \times \mathbb{S}(d) \rightarrow \mathbb{R}$ be a given function dominated by $G$ in the sense of (8.29). The construction of a $\mathbb{R}^{2 d}$-dimensional $\widetilde{G}$-Brownian motion $\left(B_{t}, b_{t}\right)_{t \geq 0}$ under a nonlinear expectation $\widetilde{\mathbb{E}}$, dominated by a sublinear expectation $\hat{\mathbb{E}}$ is based on a similar approach introduced in Section 2. In fact we will see that by our construction $\left(B_{t}, b_{t}\right)_{t \geq 0}$ is also a $G$-Brownian motion of the sublinear expectation $\hat{\mathbb{E}}$. 
We denote by $\Omega=C_{0}^{2 d}\left(\mathbb{R}^{+}\right)$the space of all $\mathbb{R}^{2 d}$-valued continuous paths $\left(\omega_{t}\right)_{t \in \mathbb{R}^{+}}$. For each fixed $T \in[0, \infty)$, we set $\Omega_{T}:=\{\omega \cdot \wedge T: \omega \in \Omega\}$. We will consider the canonical process $\left(B_{t}, b_{t}\right)(\omega)=\omega_{t}, t \in[0, \infty)$, for $\omega \in \Omega$. We also follow section 2 to introduce the spaces of random variables $L_{i p}\left(\Omega_{T}\right)$ and $L_{i p}(\Omega)$ so that to define $\hat{\mathbb{E}}$ and $\widetilde{\mathbb{E}}$ on $\left(\Omega, L_{i p}(\Omega)\right)$.

To this purpose we first construct a sequence of $d$-dimensional random vectors $\left(X_{i}, \eta_{i}\right)_{i=1}^{\infty}$ on a sublinear expectation space $(\bar{\Omega}, \overline{\mathcal{H}}, \overline{\mathbb{E}})$ such that $\left(X_{i}, \eta_{i}\right)$ is $G$-distributed and $\left(X_{i+1}, \eta_{i+1}\right)$ is independent from $\left(\left(X_{1}, \eta_{1}\right), \cdots,\left(X_{i}, \eta_{i}\right)\right)$ for each $i=1,2, \cdots$. By the definition of $G$-distribution the function

$$
u(t, x, y):=\hat{\mathbb{E}}\left[\varphi\left(x+\sqrt{t} X_{1}, y+t \eta_{1}\right)\right], \quad t \geq 0, \quad x, y \in \mathbb{R}^{d}
$$

is the viscosity solution of the following parabolic PDE, which is the same as equation (1.6) in Chap.II.

$$
\partial_{t} u-G\left(D_{y} u, D_{x x}^{2} u\right)=0,\left.\quad u\right|_{t=0}=\varphi \in C_{L i p}\left(\mathbb{R}^{2 d}\right) .
$$

We also consider the PDE (for the existence, uniqueness, comparison and domination properties, see Theorem 2.6 in Appendix C).

$$
\partial_{t} \tilde{u}-\widetilde{G}\left(D_{y} \tilde{u}, D_{x x}^{2} \tilde{u}\right)=0,\left.\quad \tilde{u}\right|_{t=0}=\varphi \in C_{L i p}\left(\mathbb{R}^{2 d}\right),
$$

and denote by $\widetilde{P}_{t}[\varphi](x, y)=\tilde{u}(t, x, y)$. Since $\widetilde{G}$ is dominated by $G$, it follows from the domination theorem of viscosity solutions, i.e., Theorem 3.5 in Appendix C, that, for each $\varphi, \psi \in C_{b, L i p}\left(\mathbb{R}^{2 d}\right)$,

$$
\widetilde{P}_{t}[\varphi](x, y)-\widetilde{P}_{t}[\psi](x, y) \leq \overline{\mathbb{E}}\left[(\varphi-\psi)\left(x+\sqrt{t} X_{1}, y+t \eta_{1}\right)\right] .
$$

We now introduce a sublinear expectation $\hat{\mathbb{E}}$ and a nonlinear $\widetilde{\mathbb{E}}$ defined on $L_{i p}(\Omega)$ via the following procedure: for each $X \in L_{i p}(\Omega)$ with

$$
X=\varphi\left(B_{t_{1}}-B_{t_{0}}, b_{t_{1}}-b_{t_{0}}, \cdots, B_{t_{1}}-B_{t_{0}}, b_{t_{n}}-b_{t_{n-1}}\right)
$$

for $\varphi \in C_{l . L i p}\left(\mathbb{R}^{2 d \times n}\right)$ and $0=t_{0}<t_{1}<\cdots<t_{n}<\infty$, we set

$$
\begin{gathered}
\hat{\mathbb{E}}\left[\varphi\left(B_{t_{1}}-B_{t_{0}}, b_{t_{1}}-b_{t_{0}}, \cdots, B_{t_{n}}-B_{t_{n-1}}, b_{t_{n}}-b_{t_{n-1}}\right)\right] \\
:=\overline{\mathbb{E}}\left[\varphi\left(\sqrt{t_{1}-t_{0}} X_{1},\left(t_{1}-t_{0}\right) \eta_{1}, \cdots, \sqrt{t_{n}-t_{n-1}} X_{n},\left(t_{n}-t_{n-1}\right) \eta_{n}\right)\right] .
\end{gathered}
$$

and

$$
\widetilde{\mathbb{E}}\left[\varphi\left(B_{t_{1}}-B_{t_{0}}, b_{t_{1}}-b_{t_{0}}, \cdots, B_{t_{n}}-B_{t_{n-1}}, b_{t_{n}}-b_{t_{n-1}}\right)\right]=\varphi_{n}(0,0)
$$

where $\varphi_{n} \in C_{b . L i p}\left(\mathbb{R}^{2 d}\right)$ is defined iteratively through

$$
\begin{aligned}
\varphi_{1}\left(x_{1}, y_{1}, \cdots, x_{n-1}, y_{n-1}\right)= & \widetilde{P}_{t_{n}-t_{n-1}}\left[\varphi_{1}\left(x_{1}, y_{1}, \cdots, x_{n-1}, y_{n-1}, \cdot\right)\right](0,0), \\
& \vdots \\
\varphi_{n-1}\left(x_{1}, y_{1}\right)= & \widetilde{P}_{t_{2}-t_{1}}\left[\varphi_{n-2}\left(x_{1}, y_{1}, \cdot\right)\right](0,0), \\
\varphi_{n}\left(x_{1}, y_{1}\right)= & \widetilde{P}_{t_{2}-t_{1}}\left[\varphi_{n-1}(\cdot)\right]\left(x_{1}, y_{1}\right) .
\end{aligned}
$$


The related conditional expectation of $X=\varphi\left(B_{t_{1}}-B_{t_{0}}, b_{t_{1}}-b_{t_{0}}, \cdots, B_{t_{n}}-\right.$ $\left.B_{t_{n-1}}, b_{t_{n}}-b_{t_{n-1}}\right)$ under $\Omega_{t_{j}}$ is defined by

$$
\begin{aligned}
\hat{\mathbb{E}}\left[X \mid \Omega_{t_{j}}\right] & =\hat{\mathbb{E}}\left[\varphi\left(B_{t_{1}}-B_{t_{0}}, b_{t_{1}}-b_{t_{0}}, \cdots, B_{t_{n}}-B_{t_{n-1}}, b_{t_{n}}-b_{t_{n-1}}\right) \mid \Omega_{t_{j}}\right] \\
& :=\psi\left(B_{t_{1}}-B_{t_{0}}, b_{t_{1}}-b_{t_{0}}, \cdots, B_{t_{j}}-B_{t_{j-1}}, b_{t_{j}}-b_{t_{j-1}}\right)
\end{aligned}
$$

where

$\psi\left(x_{1}, \cdots, x_{j}\right)=\overline{\mathbb{E}}\left[\varphi\left(x_{1}, \cdots, x_{j}, \sqrt{t_{j+1}-t_{j}} X_{j+1},\left(t_{1}-t_{0}\right) \eta_{j+1}, \cdots, \sqrt{t_{n}-t_{n-1}} X_{n},\left(t_{1}-t_{0}\right) \eta_{n}\right)\right]$.

Similarly

$$
\widetilde{\mathbb{E}}\left[X \mid \Omega_{t_{j}}\right]=\varphi_{n-j}\left(B_{t_{1}}-B_{t_{0}}, b_{t_{1}}-b_{t_{0}}, \cdots, B_{t_{j}}-B_{t_{j-1}}, b_{t_{j}}-b_{t_{j-1}}\right) .
$$

It is easy to check that $\hat{\mathbb{E}}[\cdot]$ (resp. $\widetilde{\mathbb{E}}$ ) consistently defines a sublinear (resp. nonlinear) expectation and $\widetilde{\mathbb{E}}[\cdot]$ on $\left(\Omega, L_{i p}(\Omega)\right)$. Moreover $\left(B_{t}, b_{t}\right)_{t \geq 0}$ is a $G$ Brownian motion under $\hat{\mathbb{E}}$ and a $\widetilde{G}$-Brownian motion under $\widetilde{\mathbb{E}}$.

Proposition 9.1 We also list the properties of $\widetilde{\mathbb{E}}\left[\cdot \mid \Omega_{t}\right]$ that hold for each $X, Y \in L_{i p}(\Omega)$ :

(i) If $X \geq Y$, then $\widetilde{\mathbb{E}}\left[X \mid \Omega_{t}\right] \geq \widetilde{\mathbb{E}}\left[Y \mid \Omega_{t}\right]$.

(ii) $\widetilde{\mathbb{E}}\left[X+\eta \mid \Omega_{t}\right]=\widetilde{\mathbb{E}}\left[X \mid \Omega_{t}\right]+\eta$, for each $t \geq 0$ and $\eta \in L_{i p}\left(\Omega_{t}\right)$.

(iii) $\widetilde{\mathbb{E}}\left[X \mid \Omega_{t}\right]-\widetilde{\mathbb{E}}\left[Y \mid \Omega_{t}\right] \leq \hat{\mathbb{E}}\left[X-Y \mid \Omega_{t}\right]$.

(iv) $\widetilde{\mathbb{E}}\left[\widetilde{\mathbb{E}}\left[X \mid \Omega_{t}\right] \mid \Omega_{s}\right]=\widetilde{\mathbb{E}}\left[X \mid \Omega_{t \wedge s}\right]$, in particular, $\widetilde{\mathbb{E}}\left[\widetilde{\mathbb{E}}\left[X \mid \Omega_{t}\right]\right]=\widetilde{\mathbb{E}}[X]$.

(v) For each $X \in L_{i p}\left(\Omega^{t}\right), \widetilde{\mathbb{E}}\left[X \mid \Omega_{t}\right]=\widetilde{\mathbb{E}}[X]$, where $L_{i p}\left(\Omega^{t}\right)$ is the linear space of random variables with the form

$$
\begin{aligned}
& \varphi\left(W_{t_{2}}-W_{t_{1}}, W_{t_{3}}-W_{t_{2}}, \cdots, W_{t_{n+1}}-W_{t_{n}}\right), \\
& n=1,2, \cdots, \varphi \in C_{l . L i p}\left(\mathbb{R}^{d \times n}\right), t_{1}, \cdots, t_{n}, t_{n+1} \in[t, \infty) .
\end{aligned}
$$

Since $\hat{\mathbb{E}}$ can be considered as a special nonlinear expectation of $\widetilde{\mathbb{E}}$ dominated by its self, thus $\hat{\mathbb{E}}\left[\cdot \mid \Omega_{t}\right]$ also satisfies above properties (i)-(v). Moreover

Proposition 9.2 The conditional sublinear expectation $\hat{\mathbb{E}}\left[\cdot \mid \Omega_{t}\right]$ satisfies (i)-(v). Moreover $\hat{\mathbb{E}}\left[\cdot \mid \Omega_{t}\right]$ itself is sublinear, i.e., (vi) $\hat{\mathbb{E}}\left[X \mid \Omega_{t}\right]-\hat{\mathbb{E}}\left[Y \mid \Omega_{t}\right] \leq \hat{\mathbb{E}}\left[X-Y \mid \Omega_{t}\right], \quad$.

(vii) $\hat{\mathbb{E}}\left[\eta X \mid \Omega_{t}\right]=\eta^{+} \hat{\mathbb{E}}\left[X \mid \Omega_{t}\right]+\eta^{-} \hat{\mathbb{E}}\left[-X \mid \Omega_{t}\right]$ for each $\eta \in L_{i p}\left(\Omega_{t}\right)$.

We now consider the completion of sublinear expectation space $\left(\Omega, L_{i p}(\Omega), \hat{\mathbb{E}}\right)$. We denote by $L_{G}^{p}(\Omega), p \geq 1$, the completion of $L_{i p}(\Omega)$ under the norm $\|X\|_{p}:=\left(\hat{\mathbb{E}}\left[|X|^{p}\right]\right)^{1 / p}$. Similarly, we can define $L_{G}^{p}\left(\Omega_{T}\right), L_{G}^{p}\left(\Omega_{T}^{t}\right)$ and $L_{G}^{p}\left(\Omega^{t}\right)$. It is clear that for each $0 \leq t \leq T<\infty, L_{G}^{p}\left(\Omega_{t}\right) \subseteq L_{G}^{p}\left(\Omega_{T}\right) \subseteq L_{G}^{p}(\Omega)$.

According to Sec凹in Chap $\mathbb{\mathbb { E }}[\cdot]$ can be continuously extended to $\left(\Omega, L_{G}^{1}(\Omega)\right)$. Moreover, since $\widetilde{\mathbb{E}}$ is dominated by $\hat{\mathbb{E}}$, thus by Definition 4.4 in Chap.I, $\left(\Omega, L_{G}^{1}(\Omega), \hat{\mathbb{E}}\right)$ forms a sublinear expectation space and $\left(\Omega, L_{G}^{1}(\Omega), \widetilde{\mathbb{E}}\right)$ forms a nonlinear expectation space. 
We now consider the extension of conditional $G$-expectation. For each fixed $t \leq T$, the conditional $G$-expectation $\hat{\mathbb{E}}\left[\cdot \mid \Omega_{t}\right]: L_{i p}\left(\Omega_{T}\right) \rightarrow L_{i p}\left(\Omega_{t}\right)$ is a continuous mapping under $\|\cdot\|$. Indeed, we have

$$
\widetilde{\mathbb{E}}\left[X \mid \Omega_{t}\right]-\widetilde{\mathbb{E}}\left[Y \mid \Omega_{t}\right] \leq \hat{\mathbb{E}}\left[X-Y \mid \Omega_{t}\right] \leq \hat{\mathbb{E}}\left[|X-Y| \mid \Omega_{t}\right],
$$

then

$$
\left|\widetilde{\mathbb{E}}\left[X \mid \Omega_{t}\right]-\widetilde{\mathbb{E}}\left[Y \mid \Omega_{t}\right]\right| \leq \hat{\mathbb{E}}\left[|X-Y| \mid \Omega_{t}\right]
$$

We thus obtain

$$
\left\|\widetilde{\mathbb{E}}\left[X \mid \Omega_{t}\right]-\widetilde{\mathbb{E}}\left[Y \mid \Omega_{t}\right]\right\| \leq\|X-Y\|
$$

It follows that $\widetilde{\mathbb{E}}\left[\cdot \mid \Omega_{t}\right]$ can be also extended as a continuous mapping

$$
\widetilde{\mathbb{E}}\left[\cdot \mid \Omega_{t}\right]: L_{G}^{1}\left(\Omega_{T}\right) \rightarrow L_{G}^{1}\left(\Omega_{t}\right) .
$$

If the above $T$ is not fixed, then we can obtain $\widetilde{\mathbb{E}}\left[\cdot \mid \Omega_{t}\right]: L_{G}^{1}(\Omega) \rightarrow L_{G}^{1}\left(\Omega_{t}\right)$.

Remark 9.3 The above proposition also holds for $X, Y \in L_{G}^{1}(\Omega)$. But in (iv), $\eta \in L_{G}^{1}\left(\Omega_{t}\right)$ should be bounded, since $X, Y \in L_{G}^{1}(\Omega)$ does not imply $X \cdot Y \in$ $L_{G}^{1}(\Omega)$.

In particular, we have the following independence:

$$
\widetilde{\mathbb{E}}\left[X \mid \Omega_{t}\right]=\widetilde{\mathbb{E}}[X], \quad \forall X \in L_{G}^{1}\left(\Omega^{t}\right) .
$$

We give the following definition similar to the classical one:

Definition 9.4 An n-dimensional random vector $Y \in\left(L_{G}^{1}(\Omega)\right)^{n}$ is said to be independent from $\Omega_{t}$ for some given $t$ if for each $\varphi \in C_{b \text {.Lip }}\left(\mathbb{R}^{n}\right)$ we have

$$
\widetilde{\mathbb{E}}\left[\varphi(Y) \mid \Omega_{t}\right]=\widetilde{\mathbb{E}}[\varphi(Y)]
$$

\section{Notes and Comments}

Bachelier (1900) 6] proposed Brownian motion as a model for fluctuations of the stock market, Einstein (1905) [42] used Brownian motion to give experimental confirmation of the atomic theory, and Wiener (1923) [121] gave a mathematically rigorous construction of Brownian motion. Here we follow Kolmogorov's idea (1956) [74] to construct $G$-Brownian motion by introducing infinite dimensional function space and the corresponding family of infinite dimensional sublinear distributions, instead of linear distributions in [74].

The notions of $G$-Brownian motion and the related stochastic calculus of Itô's type were firstly introduced by Peng (2006) [100] for 1-dimensional case and then in (2008) [104] for multi-dimensional situation. It is very interesting that Denis and Martini (2006) 38] studied super-pricing of contingent claims under model uncertainty of volatility. They have introduced a norm on the 
space of continuous paths $\Omega=C([0, T])$ which corresponds to our $L_{G}^{2}$-norm and developed a stochastic integral. There is no notion of nonlinear expectation and the related nonlinear distribution, such as $G$-expectation, conditional $G$ expectation, the related $G$-normal distribution and the notion of independence in their paper. But on the other hand, powerful tools in capacity theory enable them to obtain pathwise results for random variables and stochastic processes through the language of "quasi-surely" (see e.g. Dellacherie (1972) 32], Dellacherie and Meyer (1978 and 1982) [33], Feyel and de La Pradelle (1989) [48]) in place of "almost surely" in classical probability theory.

The main motivations of $G$-Brownian motion were the pricing and risk measures under volatility uncertainty in financial markets (see Avellaneda, Levy and Paras (1995) [5] and Lyons (1995) 82]). It was well-known that under volatility uncertainty the corresponding uncertain probabilities are singular from each other. This causes a serious problem for the related path analysis to treat, e.g., path-dependent derivatives, under a classical probability space. Our GBrownian motion provides a powerful tool to such type of problems.

Our new Itô's calculus for $G$-Brownian motion is of course inspired from Itô's groundbreaking work since 1942 [65] on stochastic integration, stochastic differential equations and stochastic calculus through interesting books cited in Chapter [V] Itô's formula given by Theorem 6.5] is from Peng [100], 104]. Gao (2009) [54] proved a more general Itô's formula for $G$-Brownian motion. An interesting problem is: can we get an Itô's formula in which the conditions correspond the classical one? Recently Li and Peng have solved this problem in 79].

Using nonlinear Markovian semigroup known as Nisio's semigroup (see Nisio (1976) [86]), Peng (2005) [98] studied the processes with Markovian properties under a nonlinear expectation. 


\section{Chapter IV}

\section{$G$-martingales and Jensen's Inequality}

In this chapter, we introduce the notion of $G$-martingales and the related Jensen's inequality for a new type of $G$-convex functions. Essentially different from the classical situation, " $M$ is a $G$-martingale" does not imply that "- $M$ is a $G$-martingale".

\section{$\S 1$ The Notion of $G$-martingales}

We now give the notion of $G$-martingales.

Definition 1.1 A process $\left(M_{t}\right)_{t \geq 0}$ is called a $G$-martingale (respectively, $G$ supermartingale, G-submartingale) if for each $t \in[0, \infty), M_{t} \in L_{G}^{1}\left(\Omega_{t}\right)$ and for each $s \in[0, t]$, we have

$$
\hat{\mathbb{E}}\left[M_{t} \mid \Omega_{s}\right]=M_{s} \quad\left(\text { respectively, } \leq M_{s}, \geq M_{s}\right) .
$$

Example 1.2 For each fixed $X \in L_{G}^{1}(\Omega)$, it is clear that $\left(\hat{\mathbb{E}}\left[X \mid \Omega_{t}\right]\right)_{t \geq 0}$ is a $G$-martingale.

Example 1.3 For each fixed $\mathbf{a} \in \mathbb{R}^{d}$, it is easy to check that $\left(B_{t}^{\mathbf{a}}\right)_{t \geq 0}$ and $\left(-B_{t}^{\mathbf{a}}\right)_{t \geq 0}$ are $G$-martingales. The process $\left(\left\langle B^{\mathbf{a}}\right\rangle_{t}-\sigma_{\mathbf{a a}^{T}}^{2} t\right)_{t \geq 0}$ is a $G$-martingale since

$$
\begin{aligned}
\hat{\mathbb{E}}\left[\left\langle B^{\mathbf{a}}\right\rangle_{t}-\sigma_{\mathbf{a a}^{T}}^{2} t \mid \Omega_{s}\right] & =\hat{\mathbb{E}}\left[\left\langle B^{\mathbf{a}}\right\rangle_{s}-\sigma_{\mathbf{a a}^{T}}^{2} t+\left(\left\langle B^{\mathbf{a}}\right\rangle_{t}-\left\langle B^{\mathbf{a}}\right\rangle_{s}\right) \mid \Omega_{s}\right] \\
& =\left\langle B^{\mathbf{a}}\right\rangle_{s}-\sigma_{\mathbf{a a}^{T}}^{2} t+\hat{\mathbb{E}}\left[\left\langle B^{\mathbf{a}}\right\rangle_{t}-\left\langle B^{\mathbf{a}}\right\rangle_{s}\right] \\
& =\left\langle B^{\mathbf{a}}\right\rangle_{s}-\sigma_{\mathbf{a a}^{T}}^{2} s .
\end{aligned}
$$


Similarly we can show that $\left(-\left(\left\langle B^{\mathbf{a}}\right\rangle_{t}-\sigma_{\mathbf{a a}^{T}}^{2} t\right)\right)_{t \geq 0}$ is a G-submartingale. The process $\left(\left(B_{t}^{\mathbf{a}}\right)^{2}\right)_{t \geq 0}$ is a $G$-submartingale since

$$
\begin{aligned}
\hat{\mathbb{E}}\left[\left(B_{t}^{\mathbf{a}}\right)^{2} \mid \Omega_{s}\right] & =\hat{\mathbb{E}}\left[\left(B_{s}^{\mathbf{a}}\right)^{2}+\left(B_{t}^{\mathbf{a}}-B_{s}^{\mathbf{a}}\right)^{2}+2 B_{s}^{\mathbf{a}}\left(B_{t}^{\mathbf{a}}-B_{s}^{\mathbf{a}}\right) \mid \Omega_{s}\right] \\
& =\left(B_{s}^{\mathbf{a}}\right)^{2}+\hat{\mathbb{E}}\left[\left(B_{t}^{\mathbf{a}}-B_{s}^{\mathbf{a}}\right)^{2} \mid \Omega_{s}\right] \\
& =\left(B_{s}^{\mathbf{a}}\right)^{2}+\sigma_{\mathbf{a a}^{T}}^{2}(t-s) \geq\left(B_{s}^{\mathbf{a}}\right)^{2} .
\end{aligned}
$$

Similarly we can prove that $\left(\left(B_{t}^{\mathbf{a}}\right)^{2}-\sigma_{\mathbf{a a}^{T}}^{2} t\right)_{t \geq 0}$ and $\left(\left(B_{t}^{\mathbf{a}}\right)^{2}-\left\langle B^{\mathbf{a}}\right\rangle_{t}\right)_{t \geq 0}$ are $G$-martingales.

In general, we have the following important property.

Proposition 1.4 Let $M_{0} \in \mathbb{R}, \varphi=\left(\varphi^{j}\right)_{j=1}^{d} \in M_{G}^{2}\left(0, T ; \mathbb{R}^{d}\right)$ and $\eta=\left(\eta^{i j}\right)_{i, j=1}^{d} \in$ $M_{G}^{1}(0, T ; \mathbb{S}(d))$ be given and let

$$
M_{t}=M_{0}+\int_{0}^{t} \varphi_{u}^{j} d B_{u}^{j}+\int_{0}^{t} \eta_{u}^{i j} d\left\langle B^{i}, B^{j}\right\rangle_{u}-\int_{0}^{t} 2 G\left(\eta_{u}\right) d u \text { for } t \in[0, T] .
$$

Then $M$ is a $G$-martingale. Here we still use the, i.e., the above repeated indices $i$ and $j$ imply the summation.

Proof. Since $\hat{\mathbb{E}}\left[\int_{s}^{t} \varphi_{u}^{j} d B_{u}^{j} \mid \Omega_{s}\right]=\hat{\mathbb{E}}\left[-\int_{s}^{t} \varphi_{u}^{j} d B_{u}^{j} \mid \Omega_{s}\right]=0$, we only need to prove that

$$
\bar{M}_{t}=\int_{0}^{t} \eta_{u}^{i j} d\left\langle B^{i}, B^{j}\right\rangle_{u}-\int_{0}^{t} 2 G\left(\eta_{u}\right) d u \text { for } t \in[0, T]
$$

is a $G$-martingale. It suffices to consider the case where $\eta \in M_{G}^{1,0}(0, T ; \mathbb{S}(d))$, i.e.,

$$
\eta_{t}=\sum_{k=0}^{N-1} \eta_{t_{k}} I_{\left[t_{k}, t_{k+1}\right)}(t)
$$

We have, for $s \in\left[t_{N-1}, t_{N}\right]$,

$$
\begin{aligned}
\hat{\mathbb{E}}\left[\bar{M}_{t} \mid \Omega_{s}\right] & =\bar{M}_{s}+\hat{\mathbb{E}}\left[\left(\eta_{t_{N-1}},\langle B\rangle_{t}-\langle B\rangle_{s}\right)-2 G\left(\eta_{t_{N-1}}\right)(t-s) \mid \Omega_{s}\right] \\
& =\bar{M}_{s}+\hat{\mathbb{E}}\left[\left(A,\langle B\rangle_{t}-\langle B\rangle_{s}\right)\right]_{A=\eta_{t_{N-1}}}-2 G\left(\eta_{t_{N-1}}\right)(t-s) \\
& =\bar{M}_{s}
\end{aligned}
$$

Then we can repeat this procedure backwardly to prove the result for $s \in$ $\left[0, t_{N-1}\right]$.

Corollary 1.5 Let $\eta \in M_{G}^{1}(0, T)$. Then for each fixed $\mathbf{a} \in \mathbb{R}^{d}$, we have

$$
\sigma_{-\mathbf{a a}^{T}}^{2} \hat{\mathbb{E}}\left[\int_{0}^{T}\left|\eta_{t}\right| d t\right] \leq \hat{\mathbb{E}}\left[\int_{0}^{T}\left|\eta_{t}\right| d\left\langle B^{\mathbf{a}}\right\rangle_{t}\right] \leq \sigma_{\mathbf{a a}^{T}}^{2} \hat{\mathbb{E}}\left[\int_{0}^{T}\left|\eta_{t}\right| d t\right] .
$$


Proof. For each $\xi \in M_{G}^{1}(0, T)$, by the above proposition, we have

$$
\hat{\mathbb{E}}\left[\int_{0}^{T} \xi_{t} d\left\langle B^{\mathbf{a}}\right\rangle_{t}-\int_{0}^{T} 2 G_{\mathbf{a}}\left(\xi_{t}\right) d t\right]=0
$$

where $G_{\mathbf{a}}(\alpha)=\frac{1}{2}\left(\sigma_{\mathbf{a a}^{T}}^{2} \alpha^{+}-\sigma_{-\mathbf{a a}^{T}}^{2} \alpha^{-}\right)$. Letting $\xi=|\eta|$ and $\xi=-|\eta|$, we get

$$
\begin{aligned}
\hat{\mathbb{E}}\left[\int_{0}^{T}\left|\eta_{t}\right| d\left\langle B^{\mathbf{a}}\right\rangle_{t}-\sigma_{\mathbf{a a}^{T}}^{2} \int_{0}^{T}\left|\eta_{t}\right| d t\right] & =0, \\
\hat{\mathbb{E}}\left[-\int_{0}^{T}\left|\eta_{t}\right| d\left\langle B^{\mathbf{a}}\right\rangle_{t}+\sigma_{-\mathbf{a a}^{T}}^{2} \int_{0}^{T}\left|\eta_{t}\right| d t\right] & =0 .
\end{aligned}
$$

From the sub-additivity of $G$-expectation, we can easily get the result.

Remark 1.6 It is worth to mention that for a $G$-martingale $M$, in general, $-M$ is not a $G$-martingale. But in Proposition 1.4, when $\eta \equiv 0,-M$ is still a $G$-martingale.

Exercise 1.7 (a) Let $\left(M_{t}\right)_{t \geq 0}$ be a $G$-supermartingale. Show that $\left(-M_{t}\right)_{t \geq 0}$ is a $G$-submartingale.

(b) Find a $G$-submartingale $\left(M_{t}\right)_{t \geq 0}$ such that $\left(-M_{t}\right)_{t \geq 0}$ is not a $G$-supermartingale.

Exercise 1.8 (a) Let $\left(M_{t}\right)_{t \geq 0}$ and $\left(N_{t}\right)_{t \geq 0}$ be two $G$-supermartingales. Prove that $\left(M_{t}+N_{t}\right)_{t \geq 0}$ is a $G$-supermartingale.

(b) Let $\left(M_{t}\right)_{t \geq 0}$ and $\left(-M_{t}\right)_{t \geq 0}$ be two $G$-martingales. For each $G$-submartingale (respectively, G-supermartingale) $\left(N_{t}\right)_{t \geq 0}$, prove that $\left(M_{t}+N_{t}\right)_{t \geq 0}$ is a $G$ submartingale (respectively, G-supermartingale).

\section{$\S 2$ On $G$-martingale Representation Theorem}

How to give a $G$-martingale representation theorem is still a largely open problem. Xu and Zhang (2009) 122 have obtained a martingale representation for a special 'symmetric' $G$-martingale process. A more general situation have been proved by Soner, Touzi and Zhang (preprint in private communications). Here we present the formulation of this $G$-martingale representation theorem under a very strong assumption.

In this section, we consider the generator $G: \mathbb{S}(d) \rightarrow \mathbb{R}$ satisfying the uniformly elliptic condition, i.e., there exists a $\beta>0$ such that, for each $A, \bar{A} \in \mathbb{S}(d)$ with $A \geq \bar{A}$,

$$
G(A)-G(\bar{A}) \geq \beta \operatorname{tr}[A-\bar{A}] .
$$

For each $\xi=\left(\xi^{j}\right)_{j=1}^{d} \in M_{G}^{2}\left(0, T ; \mathbb{R}^{d}\right)$ and $\eta=\left(\eta^{i j}\right)_{i, j=1}^{d} \in M_{G}^{1}(0, T ; \mathbb{S}(d))$, we use the following notations

$$
\int_{0}^{T}\left\langle\xi_{t}, d B_{t}\right\rangle:=\sum_{j=1}^{d} \int_{0}^{T} \xi_{t}^{j} d B_{t}^{j} ; \int_{0}^{T}\left(\eta_{t}, d\langle B\rangle_{t}\right):=\sum_{i, j=1}^{d} \int_{0}^{T} \eta_{t}^{i j} d\left\langle B^{i}, B^{j}\right\rangle_{t} .
$$

We first consider the representation of $\varphi\left(B_{T}-B_{t_{1}}\right)$ for $0 \leq t_{1} \leq T<\infty$. 
Lemma 2.1 Let $\xi=\varphi\left(B_{T}-B_{t_{1}}\right), \varphi \in C_{b . L i p}\left(\mathbb{R}^{d}\right)$. Then we have the following representation:

$$
\xi=\hat{\mathbb{E}}[\xi]+\int_{t_{1}}^{T}\left\langle\beta_{t}, d B_{t}\right\rangle+\int_{t_{1}}^{T}\left(\eta_{t}, d\langle B\rangle_{t}\right)-\int_{t_{1}}^{T} 2 G\left(\eta_{t}\right) d t
$$

Proof. We know that $u(t, x)=\hat{\mathbb{E}}\left[\varphi\left(x+B_{T}-B_{t}\right)\right]$ is the solution of the following PDE:

$$
\partial_{t} u+G\left(D^{2} u\right)=0 \quad(t, x) \in[0, T] \times \mathbb{R}^{d}, u(T, x)=\varphi(x) .
$$

For each $\varepsilon>0$, by the interior regularity of $u$ (see Appendix C), we have

$$
\|u\|_{C^{1+\alpha / 2,2+\alpha}\left([0, T-\varepsilon] \times \mathbb{R}^{d}\right)}<\infty \text { for some } \alpha \in(0,1) .
$$

Applying $G$-Itô's formula to $u\left(t, B_{t}-B_{t_{1}}\right)$ on $\left[t_{1}, T-\varepsilon\right]$, since $D u(t, x)$ is uniformly bounded, letting $\varepsilon \rightarrow 0$, we have

$$
\begin{aligned}
\xi= & \hat{\mathbb{E}}[\xi]+\int_{t_{1}}^{T} \partial_{t} u\left(t, B_{t}-B_{t_{1}}\right) d t+\int_{t_{1}}^{T}\left\langle D u\left(t, B_{t}-B_{t_{1}}\right), d B_{t}\right\rangle \\
& +\frac{1}{2} \int_{t_{1}}^{T}\left(D^{2} u\left(t, B_{t}-B_{t_{1}}\right), d\langle B\rangle_{t}\right) \\
= & \hat{\mathbb{E}}[\xi]+\int_{t_{1}}^{T}\left\langle D u\left(t, B_{t}-B_{t_{1}}\right), d B_{t}\right\rangle+\frac{1}{2} \int_{t_{1}}^{T}\left(D^{2} u\left(t, B_{t}-B_{t_{1}}\right), d\langle B\rangle_{t}\right) \\
& -\int_{t_{1}}^{T} G\left(D^{2} u\left(t, B_{t}-B_{t_{1}}\right)\right) d t .
\end{aligned}
$$

We now give the representation theorem of $\xi=\varphi\left(B_{t_{1}}, B_{t_{2}}-B_{t_{1}}, \cdots, B_{t_{N}}-\right.$ $\left.B_{t_{N-1}}\right)$.

Theorem 2.2 Let $\xi=\varphi\left(B_{t_{1}}, B_{t_{2}}-B_{t_{1}}, \cdots, B_{t_{N}}-B_{t_{N-1}}\right), \varphi \in C_{b . L i p}\left(\mathbb{R}^{d \times N}\right)$, $0 \leq t_{1}<t_{2}<\cdots<t_{N}=T<\infty$. Then we have the following representation:

$$
\xi=\hat{\mathbb{E}}[\xi]+\int_{0}^{T}\left\langle\beta_{t}, d B_{t}\right\rangle+\int_{0}^{T}\left(\eta_{t}, d\langle B\rangle_{t}\right)-\int_{0}^{T} 2 G\left(\eta_{t}\right) d t .
$$

Proof. We only need to prove the case $\xi=\varphi\left(B_{t_{1}}, B_{T}-B_{t_{1}}\right)$. We set, for each $(x, y) \in \mathbb{R}^{2 d}$,

$$
u(t, x, y)=\hat{\mathbb{E}}\left[\varphi\left(x, y+B_{T}-B_{t}\right)\right] ; \varphi_{1}(x)=\hat{\mathbb{E}}\left[\varphi\left(x, B_{T}-B_{t_{1}}\right)\right] .
$$

For each $x \in \mathbb{R}^{d}$, we denote $\bar{\xi}=\varphi\left(x, B_{T}-B_{t_{1}}\right)$. By Lemma 2.1, we have

$$
\begin{aligned}
\bar{\xi}= & \varphi_{1}(x)+\int_{t_{1}}^{T}\left\langle D_{y} u\left(t, x, B_{t}-B_{t_{1}}\right), d B_{t}\right\rangle+\frac{1}{2} \int_{t_{1}}^{T}\left(D_{y}^{2} u\left(t, x, B_{t}-B_{t_{1}}\right), d\langle B\rangle_{t}\right) \\
& -\int_{t_{1}}^{T} G\left(D_{y}^{2} u\left(t, x, B_{t}-B_{t_{1}}\right)\right) d t .
\end{aligned}
$$


By the definitions of the integrations of $d t, d B_{t}$ and $d\langle B\rangle_{t}$, we can replace $x$ by $B_{t_{1}}$ and get

$$
\begin{aligned}
\xi= & \varphi_{1}\left(B_{t_{1}}\right)+\int_{t_{1}}^{T}\left\langle D_{y} u\left(t, B_{t_{1}}, B_{t}-B_{t_{1}}\right), d B_{t}\right\rangle \\
& +\frac{1}{2} \int_{t_{1}}^{T}\left(D_{y}^{2} u\left(t, B_{t_{1}}, B_{t}-B_{t_{1}}\right), d\langle B\rangle_{t}\right)-\int_{t_{1}}^{T} G\left(D_{y}^{2} u\left(t, B_{t_{1}}, B_{t}-B_{t_{1}}\right)\right) d t .
\end{aligned}
$$

Applying Lemma 2.1 to $\varphi_{1}\left(B_{t_{1}}\right)$, we complete the proof.

We then immediately have the following $G$-martingale representation theorem.

Theorem 2.3 Let $\left(M_{t}\right)_{t \in[0, T]}$ be a $G$-martingale with $M_{T}=\varphi\left(B_{t_{1}}, B_{t_{2}}-B_{t_{1}}, \cdots, B_{t_{N}}-\right.$ $\left.B_{t_{N-1}}\right), \varphi \in C_{b . \text { Lip }}\left(\mathbb{R}^{d \times N}\right), 0 \leq t_{1}<t_{2}<\cdots<t_{N}=T<\infty$. Then

$$
M_{t}=\hat{\mathbb{E}}\left[M_{T}\right]+\int_{0}^{t}\left\langle\beta_{s}, d B_{s}\right\rangle+\int_{0}^{t}\left(\eta_{s}, d\langle B\rangle_{s}\right)-\int_{0}^{t} 2 G\left(\eta_{s}\right) d s, t \leq T .
$$

Proof. For $M_{T}$, by Theorem 2.2, we have

$$
M_{T}=\hat{\mathbb{E}}\left[M_{T}\right]+\int_{0}^{T}\left\langle\beta_{s}, d B_{s}\right\rangle+\int_{0}^{T}\left(\eta_{s}, d\langle B\rangle_{s}\right)-\int_{0}^{T} 2 G\left(\eta_{s}\right) d s .
$$

Taking the conditional $G$-expectation on both sides of the above equality and by Proposition 1.4, we obtain the result.

\section{$\S 3 \quad G$-convexity and Jensen's Inequality for $G-$ expectations}

A very interesting question is whether the well-known Jensen's inequality still holds for $G$-expectations.

First, we give a new notion of convexity.

Definition 3.1 A continuous function $h: \mathbb{R} \rightarrow \mathbb{R}$ is called $G$-convex if for each bounded $\xi \in L_{G}^{1}(\Omega)$, the following Jensen's inequality holds:

$$
\hat{\mathbb{E}}[h(\xi)] \geq h(\hat{\mathbb{E}}[\xi]) .
$$

In this section, we mainly consider $C^{2}$-functions.

Proposition 3.2 Let $h \in C^{2}(\mathbb{R})$. Then the following statements are equivalent:

(i) The function $h$ is $G$-convex.

(ii) For each bounded $\xi \in L_{G}^{1}(\Omega)$, the following Jensen's inequality holds:

$$
\hat{\mathbb{E}}\left[h(\xi) \mid \Omega_{t}\right] \geq h\left(\hat{\mathbb{E}}\left[\xi \mid \Omega_{t}\right]\right) \text { for } t \geq 0 .
$$


(iii) For each $\varphi \in C_{b}^{2}\left(\mathbb{R}^{d}\right)$, the following Jensen's inequality holds:

$$
\hat{\mathbb{E}}\left[h\left(\varphi\left(B_{t}\right)\right)\right] \geq h\left(\hat{\mathbb{E}}\left[\varphi\left(B_{t}\right)\right]\right) \text { for } t \geq 0 .
$$

(iv) The following condition holds for each $(y, z, A) \in \mathbb{R} \times \mathbb{R}^{d} \times \mathbb{S}(d)$ :

$$
G\left(h^{\prime}(y) A+h^{\prime \prime}(y) z z^{T}\right)-h^{\prime}(y) G(A) \geq 0 .
$$

To prove the above proposition, we need the following lemmas.

Lemma 3.3 Let $\Phi: \mathbb{R}^{d} \rightarrow \mathbb{S}(d)$ be continuous with polynomial growth. Then

$$
\lim _{\delta \downarrow 0} \hat{\mathbb{E}}\left[\int_{t}^{t+\delta}\left(\Phi\left(B_{s}\right), d\langle B\rangle_{s}\right)\right] \delta^{-1}=2 \hat{\mathbb{E}}\left[G\left(\Phi\left(B_{t}\right)\right)\right] .
$$

Proof. If $\Phi$ is a Lipschitz function, it is easy to prove that

$$
\hat{\mathbb{E}}\left[\left|\int_{t}^{t+\delta}\left(\Phi\left(B_{s}\right)-\Phi\left(B_{t}\right), d\langle B\rangle_{s}\right)\right|\right] \leq C_{1} \delta^{3 / 2},
$$

where $C_{1}$ is a constant independent of $\delta$. Thus

$$
\begin{aligned}
\lim _{\delta \downarrow 0} \hat{\mathbb{E}}\left[\int_{t}^{t+\delta}\left(\Phi\left(B_{s}\right), d\langle B\rangle_{s}\right)\right] \delta^{-1} & =\lim _{\delta \downarrow 0} \hat{\mathbb{E}}\left[\left(\Phi\left(B_{t}\right),\langle B\rangle_{t+\delta}-\langle B\rangle_{s}\right)\right] \delta^{-1} \\
& =2 \hat{\mathbb{E}}\left[G\left(\Phi\left(B_{t}\right)\right)\right] .
\end{aligned}
$$

Otherwise, we can choose a sequence of Lipschitz functions $\Phi_{N}: \mathbb{R}^{d} \rightarrow \mathbb{S}(d)$ such that

$$
\left|\Phi_{N}(x)-\Phi(x)\right| \leq \frac{C_{2}}{N}\left(1+|x|^{k}\right),
$$

where $C_{2}$ and $k$ are positive constants independent of $N$. It is easy to show that

$$
\hat{\mathbb{E}}\left[\left|\int_{t}^{t+\delta}\left(\Phi\left(B_{s}\right)-\Phi_{N}\left(B_{s}\right), d\langle B\rangle_{s}\right)\right|\right] \leq \frac{C}{N} \delta
$$

and

$$
\hat{\mathbb{E}}\left[\left|G\left(\Phi\left(B_{t}\right)\right)-G\left(\Phi_{N}\left(B_{t}\right)\right)\right|\right] \leq \frac{C}{N},
$$

where $C$ is a universal constant. Thus

$$
\begin{aligned}
& \left|\hat{\mathbb{E}}\left[\int_{t}^{t+\delta}\left(\Phi\left(B_{s}\right), d\langle B\rangle_{s}\right)\right] \delta^{-1}-2 \hat{\mathbb{E}}\left[G\left(\Phi\left(B_{t}\right)\right)\right]\right| \\
\leq & \left|\hat{\mathbb{E}}\left[\int_{t}^{t+\delta}\left(\Phi_{N}\left(B_{s}\right), d\langle B\rangle_{s}\right)\right] \delta^{-1}-2 \hat{\mathbb{E}}\left[G\left(\Phi_{N}\left(B_{t}\right)\right)\right]\right|+\frac{3 C}{N} .
\end{aligned}
$$

Then we have

$$
\underset{\delta \downarrow 0}{\limsup }\left|\hat{\mathbb{E}}\left[\int_{t}^{t+\delta}\left(\Phi\left(B_{s}\right), d\langle B\rangle_{s}\right)\right] \delta^{-1}-2 \hat{\mathbb{E}}\left[G\left(\Phi\left(B_{t}\right)\right)\right]\right| \leq \frac{3 C}{N} .
$$

Since $N$ can be arbitrarily large, we complete the proof. 
Lemma 3.4 Let $\Psi$ be a $C^{2}$-function on $\mathbb{R}^{d}$ such that $D^{2} \Psi$ satisfy polynomial growth condition. Then we have

$$
\lim _{\delta \downarrow 0}\left(\hat{\mathbb{E}}\left[\Psi\left(B_{\delta}\right)\right]-\Psi(0)\right) \delta^{-1}=G\left(D^{2} \Psi(0)\right) .
$$

Proof. Applying $G$-Itô's formula to $\Psi\left(B_{\delta}\right)$, we get

$$
\Psi\left(B_{\delta}\right)=\Psi(0)+\int_{0}^{\delta}\left\langle D \Psi\left(B_{s}\right), d B_{s}\right\rangle+\frac{1}{2} \int_{0}^{\delta}\left(D^{2} \Psi\left(B_{s}\right), d\langle B\rangle_{s}\right) .
$$

Thus we have

$$
\hat{\mathbb{E}}\left[\Psi\left(B_{\delta}\right)\right]-\Psi(0)=\frac{1}{2} \hat{\mathbb{E}}_{G}\left[\int_{0}^{\delta}\left(D^{2} \Psi\left(B_{s}\right), d\langle B\rangle_{s}\right)\right] .
$$

By Lemma 3.3, we obtain the result.

Lemma 3.5 Let $h \in C^{2}(\mathbb{R})$ and satisfy (3.2). For each $\varphi \in C_{b . \text { Lip }}\left(\mathbb{R}^{d}\right)$, let $u(t, x)$ be the solution of the $G$-heat equation:

$$
\partial_{t} u-G\left(D^{2} u\right)=0 \quad(t, x) \in[0, \infty) \times \mathbb{R}^{d}, u(0, x)=\varphi(x) .
$$

Then $\tilde{u}(t, x):=h(u(t, x))$ is a viscosity subsolution of $G$-heat equation (3.5) with initial condition $\tilde{u}(0, x)=h(\varphi(x))$.

Proof. For each $\varepsilon>0$, we denote by $u^{\varepsilon}$ the solution of the following PDE:

$$
\partial_{t} u^{\varepsilon}-G_{\varepsilon}\left(D^{2} u^{\varepsilon}\right)=0 \quad(t, x) \in[0, \infty) \times \mathbb{R}^{d}, u^{\varepsilon}(0, x)=\varphi(x),
$$

where $G_{\varepsilon}(A):=G(A)+\varepsilon \operatorname{tr}[A]$. Since $G_{\varepsilon}$ satisfies the uniformly elliptic condition, by Appendix $\mathrm{C}$, we have $u^{\varepsilon} \in C^{1,2}\left((0, \infty) \times \mathbb{R}^{d}\right)$. By simple calculation, we have

$$
\partial_{t} h\left(u^{\varepsilon}\right)=h^{\prime}\left(u^{\varepsilon}\right) \partial_{t} u^{\varepsilon}=h^{\prime}\left(u^{\varepsilon}\right) G_{\varepsilon}\left(D^{2} u^{\varepsilon}\right)
$$

and

$$
\partial_{t} h\left(u^{\varepsilon}\right)-G_{\varepsilon}\left(D^{2} h\left(u^{\varepsilon}\right)\right)=f_{\varepsilon}(t, x), h\left(u^{\varepsilon}(0, x)\right)=h(\varphi(x)),
$$

where

$$
f_{\varepsilon}(t, x)=h^{\prime}\left(u^{\varepsilon}\right) G\left(D^{2} u^{\varepsilon}\right)-G\left(D^{2} h\left(u^{\varepsilon}\right)\right)-\varepsilon h^{\prime \prime}\left(u^{\varepsilon}\right)\left|D u^{\varepsilon}\right|^{2} .
$$

Since $h$ is $G$-convex, it follows that $f_{\varepsilon} \leq-\varepsilon h^{\prime \prime}\left(u^{\varepsilon}\right)\left|D u^{\varepsilon}\right|^{2}$. We can also deduce that $\left|D u^{\varepsilon}\right|$ is uniformly bounded by the Lipschitz constant of $\varphi$. It is easy to show that $u^{\varepsilon}$ uniformly converges to $u$ as $\varepsilon \rightarrow 0$. Thus $h\left(u^{\varepsilon}\right)$ uniformly converges to $h(u)$ and $h^{\prime \prime}\left(u^{\varepsilon}\right)$ is uniformly bounded. Then we get

$$
\partial_{t} h\left(u^{\varepsilon}\right)-G_{\varepsilon}\left(D^{2} h\left(u^{\varepsilon}\right)\right) \leq C \varepsilon, h\left(u^{\varepsilon}(0, x)\right)=h(\varphi(x)),
$$

where $C$ is a constant independent of $\varepsilon$. By Appendix $\mathrm{C}$, we conclude that $h(u)$ is a viscosity subsolution. 
Proof of Proposition 3.2 , Obviously (ii) $\Longrightarrow$ (i) $\Longrightarrow$ (iii). We now prove (iii) $\Longrightarrow$ (ii). For $\xi \in L_{G}^{1}(\Omega)$ of the form

$$
\xi=\varphi\left(B_{t_{1}}, B_{t_{2}}-B_{t_{1}}, \cdots, B_{t_{n}}-B_{t_{n-1}}\right),
$$

where $\varphi \in C_{b}^{2}\left(\mathbb{R}^{d \times n}\right), 0 \leq t_{1} \leq \cdots \leq t_{n}<\infty$, by the definitions of $\hat{\mathbb{E}}[\cdot]$ and $\hat{\mathbb{E}}\left[\cdot \mid \Omega_{t}\right]$, we have

$$
\hat{\mathbb{E}}\left[h(\xi) \mid \Omega_{t}\right] \geq h\left(\hat{\mathbb{E}}\left[\xi \mid \Omega_{t}\right]\right), t \geq 0 .
$$

We then can extend this Jensen's inequality, under the norm $\|\cdot\|=\hat{\mathbb{E}}[|\cdot|]$, to each bounded $\xi \in L_{G}^{1}(\Omega)$.

(iii) $\Longrightarrow$ (iv): for each $\varphi \in C_{b}^{2}\left(\mathbb{R}^{d}\right)$, we have $\hat{\mathbb{E}}\left[h\left(\varphi\left(B_{t}\right)\right)\right] \geq h\left(\hat{\mathbb{E}}\left[\varphi\left(B_{t}\right)\right]\right)$ for each $t \geq 0$. By Lemma 3.4, we know that

$$
\lim _{\delta \downarrow 0}\left(\hat{\mathbb{E}}\left[\varphi\left(B_{\delta}\right)\right]-\varphi(0)\right) \delta^{-1}=G\left(D^{2} \varphi(0)\right)
$$

and

$$
\lim _{\delta \downarrow 0}\left(\hat{\mathbb{E}}\left[h\left(\varphi\left(B_{\delta}\right)\right)\right]-h(\varphi(0))\right) \delta^{-1}=G\left(D^{2} h(\varphi)(0)\right) .
$$

Thus we get

$$
G\left(D^{2} h(\varphi)(0)\right) \geq h^{\prime}(\varphi(0)) G\left(D^{2} \varphi(0)\right) .
$$

For each $(y, z, A) \in \mathbb{R} \times \mathbb{R}^{d} \times \mathbb{S}(d)$, we can choose a $\varphi \in C_{b}^{2}\left(\mathbb{R}^{d}\right)$ such that $\left(\varphi(0), D \varphi(0), D^{2} \varphi(0)\right)=(y, z, A)$. Thus we obtain (iv).

(iv) $\Longrightarrow\left(\right.$ iii): for each $\varphi \in C_{b}^{2}\left(\mathbb{R}^{d}\right), u(t, x)=\hat{\mathbb{E}}\left[\varphi\left(x+B_{t}\right)\right]$ (respectively, $\bar{u}(t, x)=$ $\left.\hat{\mathbb{E}}\left[h\left(\varphi\left(x+B_{t}\right)\right)\right]\right)$ solves the $G$-heat equation (3.5). By Lemma 3.5, $h(u)$ is a viscosity subsolution of $G$-heat equation (3.5). It follows from the maximum principle that $h(u(t, x)) \leq \bar{u}(t, x)$. In particular, (iii) holds.

Remark 3.6 In fact, (i) $\Longleftrightarrow($ ii $) \Longleftrightarrow$ (iii) still hold without the assumption $h \in$ $C^{2}(\mathbb{R})$.

Proposition 3.7 Let $h$ be a $G$-convex function and $X \in L_{G}^{1}(\Omega)$ be bounded. Then $Y_{t}=h\left(\hat{\mathbb{E}}\left[X \mid \Omega_{t}\right]\right), t \geq 0$, is a $G$-submartingale.

Proof. For each $s \leq t$,

$$
\hat{\mathbb{E}}\left[Y_{t} \mid \Omega_{s}\right]=\hat{\mathbb{E}}\left[h\left(\hat{\mathbb{E}}\left[X \mid \Omega_{t}\right]\right) \mid \Omega_{s}\right] \geq h\left(\hat{\mathbb{E}}\left[X \mid \Omega_{s}\right]\right)=Y_{s} .
$$

Exercise 3.8 Suppose that $G$ satisfies the uniformly elliptic condition and $h \in$ $C^{2}(\mathbb{R})$. Show that $h$ is $G$-convex if and only if $h$ is convex. 


\section{Notes and Comments}

This chapter is mainly from Peng (2007) [102].

Peng (1997) 92] introduced a filtration consistent (or time consistent, or dynamic) nonlinear expectation, called $g$-expectation, via BSDE, and then in (1999) 94 for some basic properties of the $g$-martingale such as nonlinear DoobMeyer decomposition theorem, see also Briand, Coquet, Hu, Mémin and Peng (2000) [14], Chen, Kulperger and Jiang (2003) [20], Chen and Peng (1998) 21] and (2000) [22], Coquet, $\mathrm{Hu}$, Mémin and Peng (2001) [26], and (2002) [27], Peng (1999) [94], (2004) 97], Peng and Xu (2003) [107], Rosazza (2006) [112]. Our conjecture is that all properties obtained for $g$-martingales must has its correspondence for $G$-martingale. But this conjecture is still far from being complete. Here we present some properties of $G$-martingales.

The problem $G$-martingale representation theorem has been raised as a problem in Peng (2007) 102]. In Section 2, we only give a result with very regular random variables. Some very interesting developments to this important problem can be found in Soner, Touzi and Zhang (2009) [114] and Song (2009) [116].

Under the framework of $g$-expectation, Chen, Kulperger and Jiang (2003) [20], Hu (2005) 60], Jiang and Chen (2004) [70] investigate the Jensen's inequality for $g$-expectation. Recently, Jia and Peng (2007) [68] introduced the notion of $g$-convex function and obtained many interesting properties. Certainly a $G$-convex function concerns fully nonlinear situations. 


\section{Chapter V}

\section{Stochastic Differential Equations}

In this chapter, we consider the stochastic differential equations and backward stochastic differential equations driven by $G$-Brownian motion. The conditions and proofs of existence and uniqueness of a stochastic differential equation is similar to the classical situation. However the corresponding problems for backward stochastic differential equations are not that easy, many are still open. We only give partial results to this direction.

\section{$\S 1 \quad$ Stochastic Differential Equations}

In this chapter, we denote by $\bar{M}_{G}^{p}\left(0, T ; \mathbb{R}^{n}\right), p \geq 1$, the completion of $M_{G}^{p, 0}\left(0, T ; \mathbb{R}^{n}\right)$

under the norm $\left(\int_{0}^{T} \hat{\mathbb{E}}\left[\left|\eta_{t}\right|^{p}\right] d t\right)^{1 / p}$. It is not hard to prove that $\bar{M}_{G}^{p}\left(0, T ; \mathbb{R}^{n}\right) \subseteq$ $M_{G}^{p}\left(0, T ; \mathbb{R}^{n}\right)$. We consider all the problems in the space $\bar{M}_{G}^{p}\left(0, T ; \mathbb{R}^{n}\right)$, and the sublinear expectation space $(\Omega, \mathcal{H}, \hat{\mathbb{E}})$ is fixed.

We consider the following SDE driven by a $d$-dimensional $G$-Brownian motion:

$X_{t}=X_{0}+\int_{0}^{t} b\left(s, X_{s}\right) d s+\int_{0}^{t} h_{i j}\left(s, X_{s}\right) d\left\langle B^{i}, B^{j}\right\rangle_{s}+\int_{0}^{t} \sigma_{j}\left(s, X_{s}\right) d B_{s}^{j}, t \in[0, T]$,

where the initial condition $X_{0} \in \mathbb{R}^{n}$ is a given constant, and $b, h_{i j}, \sigma_{j}$ are given functions satisfying $b(\cdot, x), h_{i j}(\cdot, x), \sigma_{j}(\cdot, x) \in \bar{M}_{G}^{2}\left(0, T ; \mathbb{R}^{n}\right)$ for each $x \in \mathbb{R}^{n}$ and the Lipschitz condition, i.e., $\left|\phi(t, x)-\phi\left(t, x^{\prime}\right)\right| \leq K\left|x-x^{\prime}\right|$, for each $t \in[0, T]$, $x, x^{\prime} \in \mathbb{R}^{n}, \phi=b, h_{i j}$ and $\sigma_{j}$, respectively. Here the horizon $[0, T]$ can be arbitrarily large. The solution is a process $X \in \bar{M}_{G}^{2}\left(0, T ; \mathbb{R}^{n}\right)$ satisfying the SDE (1.1).

We first introduce the following mapping on a fixed interval $[0, T]$ :

$$
\Lambda . \bar{M}_{G}^{2}\left(0, T ; \mathbb{R}^{n}\right) \rightarrow \bar{M}_{G}^{2}\left(0, T ; \mathbb{R}^{n}\right)
$$


by setting $\Lambda_{t}, t \in[0, T]$, with

$$
\Lambda_{t}(Y)=X_{0}+\int_{0}^{t} b\left(s, Y_{s}\right) d s+\int_{0}^{t} h_{i j}\left(s, Y_{s}\right) d\left\langle B^{i}, B^{j}\right\rangle_{s}+\int_{0}^{t} \sigma_{j}\left(s, Y_{s}\right) d B_{s}^{j} .
$$

We immediately have the following lemma.

Lemma 1.1 For each $Y, Y^{\prime} \in \bar{M}_{G}^{2}\left(0, T ; \mathbb{R}^{n}\right)$, we have the following estimate:

$$
\hat{\mathbb{E}}\left[\left|\Lambda_{t}(Y)-\Lambda_{t}\left(Y^{\prime}\right)\right|^{2}\right] \leq C \int_{0}^{t} \hat{\mathbb{E}}\left[\left|Y_{s}-Y_{s}^{\prime}\right|^{2}\right] d s, t \in[0, T],
$$

where the constant $C$ depends only on the Lipschitz constant $K$.

We now prove that SDE (1.1) has a unique solution. By multiplying $e^{-2 C t}$ on both sides of (1.2) and integrating them on $[0, T]$, it follows that

$$
\begin{aligned}
\int_{0}^{T} \hat{\mathbb{E}}\left[\left|\Lambda_{t}(Y)-\Lambda_{t}\left(Y^{\prime}\right)\right|^{2}\right] e^{-2 C t} d t & \leq C \int_{0}^{T} e^{-2 C t} \int_{0}^{t} \hat{\mathbb{E}}_{G}\left[\left|Y_{s}-Y_{s}^{\prime}\right|^{2}\right] d s d t \\
& =C \int_{0}^{T} \int_{s}^{T} e^{-2 C t} d t \hat{\mathbb{E}}\left[\left|Y_{s}-Y_{s}^{\prime}\right|^{2}\right] d s \\
& =\frac{1}{2} \int_{0}^{T}\left(e^{-2 C s}-e^{-2 C T}\right) \hat{\mathbb{E}}\left[\left|Y_{s}-Y_{s}^{\prime}\right|^{2}\right] d s .
\end{aligned}
$$

We then have

$$
\int_{0}^{T} \hat{\mathbb{E}}\left[\left|\Lambda_{t}(Y)-\Lambda_{t}\left(Y^{\prime}\right)\right|^{2}\right] e^{-2 C t} d t \leq \frac{1}{2} \int_{0}^{T} \hat{\mathbb{E}}\left[\left|Y_{t}-Y_{t}^{\prime}\right|^{2}\right] e^{-2 C t} d t .
$$

We observe that the following two norms are equivalent on $\bar{M}_{G}^{2}\left(0, T ; \mathbb{R}^{n}\right)$, i.e.,

$$
\left(\int_{0}^{T} \hat{\mathbb{E}}\left[\left|Y_{t}\right|^{2}\right] d t\right)^{1 / 2} \sim\left(\int_{0}^{T} \hat{\mathbb{E}}\left[\left|Y_{t}\right|^{2}\right] e^{-2 C t} d t\right)^{1 / 2} .
$$

From (1.3) we can obtain that $\Lambda(Y)$ is a contraction mapping. Consequently, we have the following theorem.

Theorem 1.2 There exists a unique solution $X \in \bar{M}_{G}^{2}\left(0, T ; \mathbb{R}^{n}\right)$ of the stochastic differential equation (1.1).

We now consider the following linear SDE. For simplicity, we assume that $d=1$ and $n=1$.

$X_{t}=X_{0}+\int_{0}^{t}\left(b_{s} X_{s}+\tilde{b}_{s}\right) d s+\int_{0}^{t}\left(h_{s} X_{s}+\tilde{h}_{s}\right) d\langle B\rangle_{s}+\int_{0}^{t}\left(\sigma_{s} X_{s}+\tilde{\sigma}_{s}\right) d B_{s}, t \in[0, T]$,

where $X_{0} \in \mathbb{R}$ is given, $b, h ., \sigma$. are given bounded processes in $\bar{M}_{G}^{2}(0, T ; \mathbb{R})$ and $\tilde{b} ., \tilde{h} ., \tilde{\sigma}$. are given processes in $\bar{M}_{G}^{2}(0, T ; \mathbb{R})$. By Theorem 1.2, we know that the linear SDE (1.4) has a unique solution. 
Remark 1.3 The solution of the linear SDE (1.4) is

$X_{t}=\Gamma_{t}^{-1}\left(X_{0}+\int_{0}^{t} \tilde{b}_{s} \Gamma_{s} d s+\int_{0}^{t}\left(\tilde{h}_{s}-\sigma_{s} \tilde{\sigma}_{s}\right) \Gamma_{s} d\langle B\rangle_{s}+\int_{0}^{t} \tilde{\sigma}_{s} \Gamma_{s} d B_{s}\right), t \in[0, T]$,

where $\Gamma_{t}=\exp \left(-\int_{0}^{t} b_{s} d s-\int_{0}^{t}\left(h_{s}-\frac{1}{2} \sigma_{s}^{2}\right) d\langle B\rangle_{s}-\int_{0}^{t} \sigma_{s} d B_{s}\right)$.

In particular, if $b ., h ., \sigma$. are constants and $\tilde{b} ., \tilde{h} ., \tilde{\sigma}$. are zero, then $X$ is a geometric G-Brownian motion.

Definition 1.4 We call $X$ is a geometric G-Brownian motion if

$$
X_{t}=\exp \left(\alpha t+\beta\langle B\rangle_{t}+\gamma B_{t}\right),
$$

where $\alpha, \beta, \gamma$ are constants.

Exercise 1.5 Prove that $\bar{M}_{G}^{p}\left(0, T ; \mathbb{R}^{n}\right) \subseteq M_{G}^{p}\left(0, T ; \mathbb{R}^{n}\right)$.

Exercise 1.6 Complete the proof of Lemma 1.1

\section{$\S 2 \quad$ Backward Stochastic Differential Equations}

We consider the following type of BSDE:

$$
Y_{t}=\hat{\mathbb{E}}\left[\xi+\int_{t}^{T} f\left(s, Y_{s}\right) d s+\int_{t}^{T} h_{i j}\left(s, Y_{s}\right) d\left\langle B^{i}, B^{j}\right\rangle_{s} \mid \Omega_{t}\right], \quad t \in[0, T],
$$

where $\xi \in L_{G}^{1}\left(\Omega_{T} ; \mathbb{R}^{n}\right)$ is given, and $f, h_{i j}$ are given functions satisfying $f(\cdot, y)$, $h_{i j}(\cdot, y) \in \bar{M}_{G}^{1}\left(0, T ; \mathbb{R}^{n}\right)$ for each $y \in \mathbb{R}^{n}$ and the Lipschitz condition, i.e., $\left|\phi(t, y)-\phi\left(t, y^{\prime}\right)\right| \leq K\left|y-y^{\prime}\right|$, for each $t \in[0, T], y, y^{\prime} \in \mathbb{R}^{n}, \phi=f$ and $h_{i j}$, respectively. The solution is a process $Y \in \bar{M}_{G}^{1}\left(0, T ; \mathbb{R}^{n}\right)$ satisfying the above BSDE.

We first introduce the following mapping on a fixed interval $[0, T]$ :

$$
\Lambda .: \bar{M}_{G}^{1}\left(0, T ; \mathbb{R}^{n}\right) \rightarrow \bar{M}_{G}^{1}\left(0, T ; \mathbb{R}^{n}\right)
$$

by setting $\Lambda_{t}, t \in[0, T]$, with

$$
\Lambda_{t}(Y)=\hat{\mathbb{E}}\left[\xi+\int_{t}^{T} f\left(s, Y_{s}\right) d s+\int_{t}^{T} h_{i j}\left(s, Y_{s}\right) d\left\langle B^{i}, B^{j}\right\rangle_{s} \mid \Omega_{t}\right] .
$$

We immediately have

Lemma 2.1 For each $Y, Y^{\prime} \in \bar{M}_{G}^{1}\left(0, T ; \mathbb{R}^{n}\right)$, we have the following estimate:

$$
\hat{\mathbb{E}}\left[\left|\Lambda_{t}(Y)-\Lambda_{t}\left(Y^{\prime}\right)\right|\right] \leq C \int_{t}^{T} \hat{\mathbb{E}}\left[\left|Y_{s}-Y_{s}^{\prime}\right|\right] d s, \quad t \in[0, T],
$$

where the constant $C$ depends only on the Lipschitz constant $K$. 
We now prove that BSDE (2.6) has a unique solution. By multiplying $e^{2 C t}$ on both sides of (2.7) and integrating them on $[0, T]$, it follows that

$$
\begin{aligned}
\int_{0}^{T} \hat{\mathbb{E}}\left[\left|\Lambda_{t}(Y)-\Lambda_{t}\left(Y^{\prime}\right)\right|\right] e^{2 C t} d t & \leq C \int_{0}^{T} \int_{t}^{T} \hat{\mathbb{E}}\left[\left|Y_{s}-Y_{s}^{\prime}\right|\right] e^{2 C t} d s d t \\
& =C \int_{0}^{T} \hat{\mathbb{E}}\left[\left|Y_{s}-Y_{s}^{\prime}\right|\right] \int_{0}^{s} e^{2 C t} d t d s \\
& =\frac{1}{2} \int_{0}^{T} \hat{\mathbb{E}}\left[\left|Y_{s}-Y_{s}^{\prime}\right|\right]\left(e^{2 C s}-1\right) d s \\
& \leq \frac{1}{2} \int_{0}^{T} \hat{\mathbb{E}}\left[\left|Y_{s}-Y_{s}^{\prime}\right|\right] e^{2 C s} d s
\end{aligned}
$$

We observe that the following two norms are equivalent on $\bar{M}_{G}^{1}\left(0, T ; \mathbb{R}^{n}\right)$, i.e.,

$$
\int_{0}^{T} \hat{\mathbb{E}}\left[\left|Y_{t}\right|\right] d t \sim \int_{0}^{T} \hat{\mathbb{E}}\left[\left|Y_{t}\right|\right] e^{2 C t} d t
$$

From (2.8), we can obtain that $\Lambda(Y)$ is a contraction mapping. Consequently, we have the following theorem.

Theorem 2.2 There exists a unique solution $\left(Y_{t}\right)_{t \in[0, T]} \in \bar{M}_{G}^{1}\left(0, T ; \mathbb{R}^{n}\right)$ of the backward stochastic differential equation (2.6).

Let $Y^{v}, v=1,2$, be the solutions of the following BSDE:

$$
Y_{t}^{v}=\hat{\mathbb{E}}\left[\xi^{v}+\int_{t}^{T}\left(f\left(s, Y_{s}^{v}\right)+\varphi_{s}^{v}\right) d s+\int_{t}^{T}\left(h_{i j}\left(s, Y_{s}^{v}\right)+\psi_{s}^{i j, v}\right) d\left\langle B^{i}, B^{j}\right\rangle_{s} \mid \Omega_{t}\right] .
$$

Then the following estimate holds.

Proposition 2.3 We have

$$
\hat{\mathbb{E}}\left[\left|Y_{t}^{1}-Y_{t}^{2}\right|\right] \leq C e^{C(T-t)}\left(\hat{\mathbb{E}}\left[\left|\xi^{1}-\xi^{2}\right|\right]+\int_{t}^{T} \hat{\mathbb{E}}\left[\left|\varphi_{s}^{1}-\varphi_{s}^{2}\right|+\left|\psi_{s}^{i j, 1}-\psi_{s}^{i j, 2}\right|\right] d s\right),
$$

where the constant $C$ depends only on the Lipschitz constant $K$.

Proof. Similar to Lemma 2.1, we have

$$
\begin{aligned}
\hat{\mathbb{E}}\left[\left|Y_{t}^{1}-Y_{t}^{2}\right|\right] \leq & C\left(\int_{t}^{T} \hat{\mathbb{E}}\left[\left|Y_{s}^{1}-Y_{s}^{2}\right|\right] d s+\hat{\mathbb{E}}\left[\left|\xi^{1}-\xi^{2}\right|\right]\right. \\
& \left.+\int_{t}^{T} \hat{\mathbb{E}}\left[\left|\varphi_{s}^{1}-\varphi_{s}^{2}\right|+\left|\psi_{s}^{i j, 1}-\psi_{s}^{i j, 2}\right|\right] d s\right) .
\end{aligned}
$$

By the Gronwall inequality (see Exercise 2.5), we conclude the result. 
Remark 2.4 In particular, if $\xi^{2}=0, \varphi_{s}^{2}=-f(s, 0), \psi_{s}^{i j, 2}=-h_{i j}(s, 0), \varphi_{s}^{1}=$ $0, \psi_{s}^{i j, 1}=0$, we obtain the estimate of the solution of the BSDE. Let $Y$ be the solution of the BSDE (2.6). Then

$$
\hat{\mathbb{E}}\left[\left|Y_{t}\right|\right] \leq C e^{C(T-t)}\left(\hat{\mathbb{E}}[|\xi|]+\int_{t}^{T} \hat{\mathbb{E}}\left[|f(s, 0)|+\left|h_{i j}(s, 0)\right|\right] d s\right),
$$

where the constant $C$ depends only on the Lipschitz constant $K$.

Exercise 2.5 (The Gronwall inequality) Let $u(t)$ be a nonnegative function such that

$$
u(t) \leq C+A \int_{0}^{t} u(s) d s \text { for } 0 \leq t \leq T,
$$

where $C$ and $A$ are constants. Prove that $u(t) \leq C e^{A t}$ for $0 \leq t \leq T$.

Exercise 2.6 For each $\xi \in L_{G}^{1}\left(\Omega_{T} ; \mathbb{R}^{n}\right)$. Show that the process $\left(\hat{\mathbb{E}}\left[\xi \mid \Omega_{t}\right]\right)_{t \in[0, T]}$ belongs to $\bar{M}_{G}^{1}\left(0, T ; \mathbb{R}^{n}\right)$.

Exercise 2.7 Complete the proof of Lemma 2.1

\section{$\S 3 \quad$ Nonlinear Feynman-Kac Formula}

Consider the following SDE:

$$
\left\{\begin{aligned}
d X_{s}^{t, \xi} & =b\left(X_{s}^{t, \xi}\right) d s+h_{i j}\left(X_{s}^{t, \xi}\right) d\left\langle B^{i}, B^{j}\right\rangle_{s}+\sigma_{j}\left(X_{s}^{t, \xi}\right) d B_{s}^{j}, s \in[t, T], \\
X_{t}^{t, \xi} & =\xi
\end{aligned}\right.
$$

where $\xi \in L_{G}^{2}\left(\Omega_{t} ; \mathbb{R}^{n}\right)$ is given and $b, h_{i j}, \sigma_{j}: \mathbb{R}^{n} \rightarrow \mathbb{R}^{n}$ are given Lipschitz functions, i.e., $\left|\phi(x)-\phi\left(x^{\prime}\right)\right| \leq K\left|x-x^{\prime}\right|$, for each $x, x^{\prime} \in \mathbb{R}^{n}, \phi=b, h_{i j}$ and $\sigma_{j}$.

We then consider associated BSDE:

$$
Y_{s}^{t, \xi}=\hat{\mathbb{E}}\left[\Phi\left(X_{T}^{t, \xi}\right)+\int_{s}^{T} f\left(X_{r}^{t, \xi}, Y_{r}^{t, \xi}\right) d r+\int_{s}^{T} g_{i j}\left(X_{r}^{t, \xi}, Y_{r}^{t, \xi}\right) d\left\langle B^{i}, B^{j}\right\rangle_{r} \mid \Omega_{s}\right],
$$

where $\Phi: \mathbb{R}^{n} \rightarrow \mathbb{R}$ is a given Lipschitz function and $f, g_{i j}: \mathbb{R}^{n} \times \mathbb{R} \rightarrow \mathbb{R}$ are given Lipschitz functions, i.e., $\left|\phi(x, y)-\phi\left(x^{\prime}, y^{\prime}\right)\right| \leq K\left(\left|x-x^{\prime}\right|+\left|y-y^{\prime}\right|\right)$, for each $x, x^{\prime} \in \mathbb{R}^{n}, y, y^{\prime} \in \mathbb{R}, \phi=f$ and $g_{i j}$.

We have the following estimates:

Proposition 3.1 For each $\xi, \xi^{\prime} \in L_{G}^{2}\left(\Omega_{t} ; \mathbb{R}^{n}\right)$, we have, for each $s \in[t, T]$,

$$
\hat{\mathbb{E}}\left[\left|X_{s}^{t, \xi}-X_{s}^{t, \xi^{\prime}}\right|^{2} \mid \Omega_{t}\right] \leq C\left|\xi-\xi^{\prime}\right|^{2}
$$

and

$$
\hat{\mathbb{E}}\left[\left|X_{s}^{t, \xi}\right|^{2} \mid \Omega_{t}\right] \leq C\left(1+|\xi|^{2}\right),
$$

where the constant $C$ depends only on the Lipschitz constant $K$. 
Proof. It is easy to obtain

$$
\hat{\mathbb{E}}\left[\left|X_{s}^{t, \xi}-X_{s}^{t, \xi^{\prime}}\right|^{2} \mid \Omega_{t}\right] \leq C_{1}\left(\left|\xi-\xi^{\prime}\right|^{2}+\int_{t}^{s} \hat{\mathbb{E}}\left[\left|X_{r}^{t, \xi}-X_{r}^{t, \xi^{\prime}}\right|^{2} \mid \Omega_{t}\right] d r\right) .
$$

By the Gronwall inequality, we obtain

$$
\hat{\mathbb{E}}\left[\left|X_{s}^{t, \xi}-X_{s}^{t, \xi^{\prime}}\right|^{2} \mid \Omega_{t}\right] \leq C_{1} e^{C_{1} T}\left|\xi-\xi^{\prime}\right|^{2} .
$$

Similarly, we can get (3.14).

Corollary 3.2 For each $\xi \in L_{G}^{2}\left(\Omega_{t} ; \mathbb{R}^{n}\right)$, we have

$$
\hat{\mathbb{E}}\left[\left|X_{t+\delta}^{t, \xi}-\xi\right|^{2} \mid \Omega_{t}\right] \leq C\left(1+|\xi|^{2}\right) \delta \text { for } \delta \in[0, T-t],
$$

where the constant $C$ depends only on the Lipschitz constant $K$.

Proof. It is easy to obtain

$$
\hat{\mathbb{E}}\left[\left|X_{t+\delta}^{t, \xi}-\xi\right|^{2} \mid \Omega_{t}\right] \leq C_{1} \int_{t}^{t+\delta}\left(1+\hat{\mathbb{E}}\left[\left|X_{s}^{t, \xi}\right|^{2} \mid \Omega_{t}\right]\right) d s .
$$

By Proposition 3.1 we obtain the result.

Proposition 3.3 For each $\xi, \xi^{\prime} \in L_{G}^{2}\left(\Omega_{t} ; \mathbb{R}^{n}\right)$, we have

$$
\left|Y_{t}^{t, \xi}-Y_{t}^{t, \xi^{\prime}}\right| \leq C\left|\xi-\xi^{\prime}\right|
$$

and

$$
\left|Y_{t}^{t, \xi}\right| \leq C(1+|\xi|)
$$

where the constant $C$ depends only on the Lipschitz constant $K$.

Proof. For each $s \in[0, T]$, it is easy to check that

$$
\left|Y_{s}^{t, \xi}-Y_{s}^{t, \xi^{\prime}}\right| \leq C_{1} \hat{\mathbb{E}}\left[\left|X_{T}^{t, \xi}-X_{T}^{t, \xi^{\prime}}\right|+\int_{s}^{T}\left(\left|X_{r}^{t, \xi}-X_{r}^{t, \xi^{\prime}}\right|+\left|Y_{r}^{t, \xi}-Y_{r}^{t, \xi^{\prime}}\right|\right) d r \mid \Omega_{s}\right] .
$$

Since

$$
\hat{\mathbb{E}}\left[\left|X_{s}^{t, \xi}-X_{s}^{t, \xi^{\prime}}\right| \mid \Omega_{t}\right] \leq\left(\hat{\mathbb{E}}\left[\left|X_{s}^{t, \xi}-X_{s}^{t, \xi^{\prime}}\right|^{2} \mid \Omega_{t}\right]\right)^{1 / 2},
$$

we have

$$
\hat{\mathbb{E}}\left[\left|Y_{s}^{t, \xi}-Y_{s}^{t, \xi^{\prime}}\right| \mid \Omega_{t}\right] \leq C_{2}\left(\left|\xi-\xi^{\prime}\right|+\int_{s}^{T} \hat{\mathbb{E}}\left[\left|Y_{r}^{t, \xi}-Y_{r}^{t, \xi^{\prime}}\right| \mid \Omega_{t}\right] d r\right) .
$$

By the Gronwall inequality, we obtain (3.16). Similarly we can get (3.17).

We are more interested in the case when $\xi=x \in \mathbb{R}^{n}$. Define

$$
u(t, x):=Y_{t}^{t, x}, \quad(t, x) \in[0, T] \times \mathbb{R}^{n} .
$$

By the above proposition, we immediately have the following estimates:

$$
\begin{gathered}
\left|u(t, x)-u\left(t, x^{\prime}\right)\right| \leq C\left|x-x^{\prime}\right|, \\
|u(t, x)| \leq C(1+|x|),
\end{gathered}
$$

where the constant $C$ depends only on the Lipschitz constant $K$. 
Remark 3.4 It is important to note that $u(t, x)$ is a deterministic function of $(t, x)$, because $X_{s}^{t, x}$ and $Y_{s}^{t, x}$ are independent from $\Omega_{t}$.

Theorem 3.5 For each $\xi \in L_{G}^{2}\left(\Omega_{t} ; \mathbb{R}^{n}\right)$, we have

$$
u(t, \xi)=Y_{t}^{t, \xi} \text {. }
$$

Proposition 3.6 We have, for $\delta \in[0, T-t]$,

$u(t, x)=\hat{\mathbb{E}}\left[u\left(t+\delta, X_{t+\delta}^{t, x}\right)+\int_{t}^{t+\delta} f\left(X_{r}^{t, x}, Y_{r}^{t, x}\right) d r+\int_{t}^{t+\delta} g_{i j}\left(X_{r}^{t, x}, Y_{r}^{t, x}\right) d\left\langle B^{i}, B^{j}\right\rangle_{r}\right]$.

Proof. Since $X_{s}^{t, x}=X_{s}^{t+\delta, X_{t+\delta}^{t, x}}$ for $s \in[t+\delta, T]$, we get $Y_{t+\delta}^{t, x}=Y_{t+\delta}^{t+\delta, X_{t+\delta}^{t, x}}$. By Theorem 3.5, we have $Y_{t+\delta}^{t, x}=u\left(t+\delta, X_{t+\delta}^{t, x}\right)$, which implies the result.

For each $A \in \mathbb{S}(n), p \in \mathbb{R}^{n}, r \in \mathbb{R}$, we set

$$
F(A, p, r, x):=G(B(A, p, r, x))+\langle p, b(x)\rangle+f(x, r),
$$

where $B(A, p, r, x)$ is a $d \times d$ symmetric matrix with

$$
B_{i j}(A, p, r, x):=\left\langle A \sigma_{i}(x), \sigma_{j}(x)\right\rangle+\left\langle p, h_{i j}(x)+h_{j i}(x)\right\rangle+g_{i j}(x, r)+g_{j i}(x, r) .
$$

Theorem $3.7 u(t, x)$ is a viscosity solution of the following PDE:

$$
\left\{\begin{array}{l}
\partial_{t} u+F\left(D^{2} u, D u, u, x\right)=0 \\
u(T, x)=\Phi(x)
\end{array}\right.
$$

Proof. We first show that $u$ is a continuous function. By (3.19) we know that $u$ is a Lipschitz function in $x$. It follows from (2.10) and (3.14) that for $s \in[t, T]$, $\hat{\mathbb{E}}\left[\left|Y_{s}^{t, x}\right|\right] \leq C(1+|x|)$. Noting (3.15) and (3.22), we get $|u(t, x)-u(t+\delta, x)| \leq$ $C(1+|x|)\left(\delta^{1 / 2}+\delta\right)$ for $\delta \in[0, T-t]$. Thus $u$ is $\frac{1}{2}$-Hölder continuous in $t$, which implies that $u$ is a continuous function. We can also show, that for each $p \geq 2$,

$$
\hat{\mathbb{E}}\left[\left|X_{t+\delta}^{t, x}-x\right|^{p}\right] \leq C\left(1+|x|^{p}\right) \delta^{p / 2},
$$

Now for fixed $(t, x) \in(0, T) \times \mathbb{R}^{n}$, let $\psi \in C_{b}^{2,3}\left([0, T] \times \mathbb{R}^{n}\right)$ be such that $\psi \geq u$ and $\psi(t, x)=u(t, x)$. By (3.22), (3.24) and Taylor's expansion, it follows that, for $\delta \in(0, T-t)$,

$$
\begin{aligned}
0 \leq & \hat{\mathbb{E}}\left[\psi\left(t+\delta, X_{t+\delta}^{t, x}\right)-\psi(t, x)+\int_{t}^{t+\delta} f\left(X_{r}^{t, x}, Y_{r}^{t, x}\right) d r\right. \\
& \left.+\int_{t}^{t+\delta} g_{i j}\left(X_{r}^{t, x}, Y_{r}^{t, x}\right) d\left\langle B^{i}, B^{j}\right\rangle_{r}\right] \\
\leq & \frac{1}{2} \hat{\mathbb{E}}\left[\left(B\left(D^{2} \psi(t, x), D \psi(t, x), \psi(t, x), x\right),\langle B\rangle_{t+\delta}-\langle B\rangle_{t}\right)\right] \\
& +\left(\partial_{t} \psi(t, x)+\langle D \psi(t, x), b(x)\rangle+f(x, \psi(t, x))\right) \delta+C\left(1+|x|+|x|^{2}+|x|^{3}\right) \delta^{3 / 2} \\
\leq & \left(\partial_{t} \psi(t, x)+F\left(D^{2} \psi(t, x), D \psi(t, x), \psi(t, x), x\right)\right) \delta+C\left(1+|x|+|x|^{2}+|x|^{3}\right) \delta^{3 / 2},
\end{aligned}
$$


then it is easy to check that

$$
\partial_{t} \psi(t, x)+F\left(D^{2} \psi(t, x), D \psi(t, x), \psi(t, x), x\right) \geq 0 .
$$

Thus $u$ is a viscosity subsolution of (3.23). Similarly we can prove that $u$ is a viscosity supersolution of (3.23).

Example 3.8 Let $B=\left(B^{1}, B^{2}\right)$ be a 2-dimensional $G$-Brownian motion with

$$
G(A)=G_{1}\left(a_{11}\right)+G_{2}\left(a_{22}\right),
$$

where

$$
G_{i}(a)=\frac{1}{2}\left(\bar{\sigma}_{i}^{2} a^{+}-\underline{\sigma}_{i}^{2} a^{-}\right), \quad i=1,2 .
$$

In this case, we consider the following 1-dimensional SDE:

$$
d X_{s}^{t, x}=\mu X_{s}^{t, x} d s+\nu X_{s}^{t, x} d\left\langle B^{1}\right\rangle_{s}+\sigma X_{s}^{t, x} d B_{s}^{2}, \quad X_{t}^{t, x}=x,
$$

where $\mu, \nu$ and $\sigma$ are constants.

The corresponding function $u$ is defined by

$$
u(t, x):=\hat{\mathbb{E}}\left[\varphi\left(X_{T}^{t, x}\right)\right]
$$

Then

$$
u(t, x)=\hat{\mathbb{E}}\left[u\left(t+\delta, X_{t+\delta}^{t, x}\right)\right]
$$

and $u$ is the viscosity solution of the following PDE:

$$
\partial_{t} u+\mu x \partial_{x} u+2 G_{1}\left(\nu x \partial_{x} u\right)+\sigma^{2} x^{2} G_{2}\left(\partial_{x x}^{2} u\right)=0, u(T, x)=\varphi(x) .
$$

Exercise 3.9 For each $\xi \in L_{G}^{p}\left(\Omega_{t} ; \mathbb{R}^{n}\right)$ with $p \geq 2$, show that $S D E$ (3.11) has a unique solution in $\bar{M}_{G}^{p}\left(t, T ; \mathbb{R}^{n}\right)$. Furthermore, show that the following estimates hold.

$$
\begin{gathered}
\hat{\mathbb{E}}\left[\left|X_{s}^{t, x}-X_{s}^{t, x^{\prime}}\right|^{p}\right] \leq C\left|x-x^{\prime}\right|^{p}, \\
\hat{\mathbb{E}}\left[\left|X_{s}^{t, x}\right|^{p}\right] \leq C\left(1+|x|^{p}\right), \\
\hat{\mathbb{E}}\left[\left|X_{t+\delta}^{t, x}-x\right|^{p}\right] \leq C\left(1+|x|^{p}\right) \delta^{p / 2} .
\end{gathered}
$$

\section{Notes and Comments}

This chapter is mainly from Peng (2007) [102].

There are many excellent books on Itô's stochastic calculus and stochastic differential equations founded by Itô's original paper [65], as well as on martingale theory. Readers are referred to Chung and Williams (1990) 25], Dellacherie and Meyer (1978 and 1982) [33], He, Wang and Yan (1992) [57], Itô and McKean (1965) 66], Ikeda and Watanabe (1981) [63], Kallenberg (2002) 72], Karatzas and Shreve (1988) [73], Øksendal (1998) [87], Protter (1990) 110], Revuz and Yor (1999) [11]] and Yong and Zhou (1999) [124]. 
Linear backward stochastic differential equation (BSDE) was first introduced by Bismut in (1973) 12] and (1978) 13]. Bensoussan developed this approach in (1981) [10] and (1982) [11]. The existence and uniqueness theorem of a general nonlinear BSDE, was obtained in 1990 in Pardoux and Peng [88]. The present version of the proof was based on El Karoui, Peng and Quenez (1997) [44], which is also a very good survey on BSDE theory and its applications, specially in finance. Comparison theorem of BSDEs was obtained in Peng (1992) 90] for the case when $g$ is a $C^{1}$-function and then in [44] when $g$ is Lipschitz. Nonlinear Feynman-Kac formula for BSDE was introduced by Peng (1991) 89] and (1992) 91. Here we obtain the corresponding Feynman-Kac formula under the framework of $G$-expectation. We also refer to Yong and Zhou (1999) [124], as well as in Peng (1997) [93] (in 1997, in Chinese) and (2004) 95] for systematic presentations of BSDE theory. For contributions in the developments of this theory, readers can be referred to the literatures listing in the Notes and Comments in Chap. [] 


\section{Chapter VI}

\section{Capacity and Quasi-Surely Analysis for $G$-Brownian Paths}

In this chapter, we first present a general framework for an upper expectation defined on a metric space $(\Omega, \mathcal{B}(\Omega))$ and the corresponding capacity to introduce the quasi-surely analysis. The results are important for us to obtain the pathwise analysis for $G$-Brownian motion.

\section{$\S 1$ Integration Theory associated to an Upper Probability}

Let $\Omega$ be a complete separable metric space equipped with the distance $d, \mathcal{B}(\Omega)$ the Borel $\sigma$-algebra of $\Omega$ and $\mathcal{M}$ the collection of all probability measures on $(\Omega, \mathcal{B}(\Omega))$.

- $L^{0}(\Omega)$ : the space of all $\mathcal{B}(\Omega)$-measurable real functions;

- $B_{b}(\Omega)$ : all bounded functions in $L^{0}(\Omega)$;

- $C_{b}(\Omega)$ : all continuous functions in $B_{b}(\Omega)$.

All along this section, we consider a given subset $\mathcal{P} \subseteq \mathcal{M}$.

\subsection{Capacity associated to $\mathcal{P}$}

We denote

$$
c(A):=\sup _{P \in \mathcal{P}} P(A), \quad A \in \mathcal{B}(\Omega) .
$$

One can easily verify the following theorem. 
Theorem 1.1 The set function $c(\cdot)$ is a Choquet capacity, i.e. (see [24, 32]),

1. $0 \leq c(A) \leq 1, \quad \forall A \subset \Omega$.

2. If $A \subset B$, then $c(A) \leq c(B)$.

3. If $\left(A_{n}\right)_{n=1}^{\infty}$ is a sequence in $\mathcal{B}(\Omega)$, then $c\left(\cup A_{n}\right) \leq \sum c\left(A_{n}\right)$.

4. If $\left(A_{n}\right)_{n=1}^{\infty}$ is an increasing sequence in $\mathcal{B}(\Omega): A_{n} \uparrow A=\cup A_{n}$, then $c\left(\cup A_{n}\right)=\lim _{n \rightarrow \infty} c\left(A_{n}\right)$.

Furthermore, we have

Theorem 1.2 For each $A \in \mathcal{B}(\Omega)$, we have

$$
c(A)=\sup \{c(K): K \text { compact } K \subset A\} .
$$

Proof. It is simply because

$$
c(A)=\sup _{P \in \mathcal{P}} \sup _{\substack{K \text { compact } \\ K \subset A}} P(K)=\sup _{\substack{K \text { compact } \\ K \subset A}} \sup _{P \in \mathcal{P}} P(K)=\sup _{\substack{K \text { compact } \\ K \subset A}} c(K) .
$$

Definition 1.3 We use the standard capacity-related vocabulary: a set $A$ is polar if $c(A)=0$ and a property holds "quasi-surely" (q.s.) if it holds outside a polar set.

Remark 1.4 In other words, $A \in \mathcal{B}(\Omega)$ is polar if and only if $P(A)=0$ for any $P \in \mathcal{P}$.

We also have in a trivial way a Borel-Cantelli Lemma.

Lemma 1.5 Let $\left(A_{n}\right)_{n \in \mathbb{N}}$ be a sequence of Borel sets such that

$$
\sum_{n=1}^{\infty} c\left(A_{n}\right)<\infty .
$$

Then $\lim \sup _{n \rightarrow \infty} A_{n}$ is polar.

Proof. Applying the Borel-Cantelli Lemma under each probability $P \in \mathcal{P}$. The following theorem is Prokhorov's theorem.

Theorem 1.6 $\mathcal{P}$ is relatively compact if and only if for each $\varepsilon>0$, there exists a compact set $K$ such that $c\left(K^{c}\right)<\varepsilon$.

The following two lemmas can be found in [62].

Lemma 1.7 $\mathcal{P}$ is relatively compact if and only if for each sequence of closed sets $F_{n} \downarrow \emptyset$, we have $c\left(F_{n}\right) \downarrow 0$. 
Proof. We outline the proof for the convenience of readers.

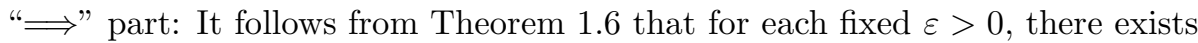
a compact set $K$ such that $c\left(K^{c}\right)<\varepsilon$. Note that $F_{n} \cap K \downarrow \emptyset$, then there exists an $N>0$ such that $F_{n} \cap K=\emptyset$ for $n \geq N$, which implies $\lim _{n} c\left(F_{n}\right)<\varepsilon$. Since $\varepsilon$ can be arbitrarily small, we obtain $c\left(F_{n}\right) \downarrow 0$.

"£" part: For each $\varepsilon>0$, let $\left(A_{i}^{k}\right)_{i=1}^{\infty}$ be a sequence of open balls of radius $1 / k$ covering $\Omega$. Observe that $\left(\cup_{i=1}^{n} A_{i}^{k}\right)^{c} \downarrow \emptyset$, then there exists an $n_{k}$ such that $c\left(\left(\cup_{i=1}^{n_{k}} A_{i}^{k}\right)^{c}\right)<\varepsilon 2^{-k}$. Set $K=\overline{\cap_{k=1}^{\infty} \cup_{i=1}^{n_{k}} A_{i}^{k}}$. It is easy to check that $K$ is compact and $c\left(K^{c}\right)<\varepsilon$. Thus by Theorem 1.6, $\mathcal{P}$ is relatively compact.

Lemma 1.8 Let $\mathcal{P}$ be weakly compact. Then for each sequence of closed sets $F_{n} \downarrow F$, we have $c\left(F_{n}\right) \downarrow c(F)$.

Proof. We outline the proof for the convenience of readers. For each fixed $\varepsilon>0$, by the definition of $c\left(F_{n}\right)$, there exists a $P_{n} \in \mathcal{P}$ such that $P_{n}\left(F_{n}\right) \geq c\left(F_{n}\right)-\varepsilon$. Since $\mathcal{P}$ is weakly compact, there exist $P_{n_{k}}$ and $P \in \mathcal{P}$ such that $P_{n_{k}}$ converge weakly to $P$. Thus

$$
P\left(F_{m}\right) \geq \limsup _{k \rightarrow \infty} P_{n_{k}}\left(F_{m}\right) \geq \limsup _{k \rightarrow \infty} P_{n_{k}}\left(F_{n_{k}}\right) \geq \lim _{n \rightarrow \infty} c\left(F_{n}\right)-\varepsilon .
$$

Letting $m \rightarrow \infty$, we get $P(F) \geq \lim _{n \rightarrow \infty} c\left(F_{n}\right)-\varepsilon$, which yields $c\left(F_{n}\right) \downarrow c(F)$.

Following [62] (see also [35, 50]) the upper expectation of $\mathcal{P}$ is defined as follows: for each $X \in L^{0}(\Omega)$ such that $E_{P}[X]$ exists for each $P \in \mathcal{P}$,

$$
\mathbb{E}[X]=\mathbb{E}^{\mathcal{P}}[X]:=\sup _{P \in \mathcal{P}} E_{P}[X] .
$$

It is easy to verify

Theorem 1.9 The upper expectation $\mathbb{E}[\cdot]$ of the family $\mathcal{P}$ is a sublinear expectation on $B_{b}(\Omega)$ as well as on $C_{b}(\Omega)$, i.e.,

1. for all $X, Y$ in $B_{b}(\Omega), X \geq Y \Longrightarrow \mathbb{E}[X] \geq \mathbb{E}[Y]$.

2. for all $X, Y$ in $B_{b}(\Omega), \mathbb{E}[X+Y] \leq \mathbb{E}[X]+\mathbb{E}[Y]$.

3. for all $\lambda \geq 0, X \in B_{b}(\Omega), \mathbb{E}[\lambda X]=\lambda \mathbb{E}[X]$.

4. for all $c \in \mathbb{R}, X \in B_{b}(\Omega), \mathbb{E}[X+c]=\mathbb{E}[X]+c$.

Moreover, it is also easy to check

Theorem 1.10 We have

1. Let $\mathbb{E}\left[X_{n}\right]$ and $\mathbb{E}\left[\sum_{n=1}^{\infty} X_{n}\right]$ be finite. Then $\mathbb{E}\left[\sum_{n=1}^{\infty} X_{n}\right] \leq \sum_{n=1}^{\infty} \mathbb{E}\left[X_{n}\right]$.

2. Let $X_{n} \uparrow X$ and $\mathbb{E}\left[X_{n}\right], \mathbb{E}[X]$ be finite. Then $\mathbb{E}\left[X_{n}\right] \uparrow \mathbb{E}[X]$.

Definition 1.11 The functional $\mathbb{E}[\cdot]$ is said to be regular if for each $\left\{X_{n}\right\}_{n=1}^{\infty}$ in $C_{b}(\Omega)$ such that $X_{n} \downarrow 0$ on $\Omega$, we have $\mathbb{E}\left[X_{n}\right] \downarrow 0$. 
Similar to Lemma 1.7 we have:

Theorem $1.12 \mathbb{E}[\cdot]$ is regular if and only if $\mathcal{P}$ is relatively compact.

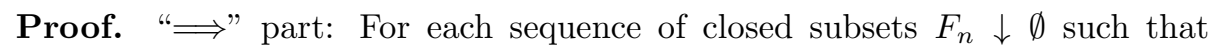
$F_{n}, n=1,2, \cdots$, are non-empty (otherwise the proof is trivial), there exists $\left\{g_{n}\right\}_{n=1}^{\infty} \subset C_{b}(\Omega)$ satisfying

$$
0 \leq g_{n} \leq 1, \quad g_{n}=1 \text { on } F_{n} \text { and } g_{n}=0 \text { on }\left\{\omega \in \Omega: d\left(\omega, F_{n}\right) \geq \frac{1}{n}\right\} .
$$

We set $f_{n}=\wedge_{i=1}^{n} g_{i}$, it is clear that $f_{n} \in C_{b}(\Omega)$ and $\mathbf{1}_{F_{n}} \leq f_{n} \downarrow 0$. $\mathbb{E}[\cdot]$ is regular implies $\mathbb{E}\left[f_{n}\right] \downarrow 0$ and thus $c\left(F_{n}\right) \downarrow 0$. It follows from Lemma 1.7 that $\mathcal{P}$ is relatively compact.

"£" part: For each $\left\{X_{n}\right\}_{n=1}^{\infty} \subset C_{b}(\Omega)$ such that $X_{n} \downarrow 0$, we have

$$
\mathbb{E}\left[X_{n}\right]=\sup _{P \in \mathcal{P}} E_{P}\left[X_{n}\right]=\sup _{P \in \mathcal{P}} \int_{0}^{\infty} P\left(\left\{X_{n} \geq t\right\}\right) d t \leq \int_{0}^{\infty} c\left(\left\{X_{n} \geq t\right\}\right) d t .
$$

For each fixed $t>0,\left\{X_{n} \geq t\right\}$ is a closed subset and $\left\{X_{n} \geq t\right\} \downarrow \emptyset$ as $n \uparrow \infty$. By Lemma 1.7 $c\left(\left\{X_{n} \geq t\right\}\right) \downarrow 0$ and thus $\int_{0}^{\infty} c\left(\left\{X_{n} \geq t\right\}\right) d t \downarrow 0$. Consequently $\mathbb{E}\left[X_{n}\right] \downarrow 0$.

\subsection{Functional spaces}

We set, for $p>0$,

- $\mathcal{L}^{p}:=\left\{X \in L^{0}(\Omega): \mathbb{E}\left[|X|^{p}\right]=\sup _{P \in \mathcal{P}} E_{P}\left[|X|^{p}\right]<\infty\right\}$;

- $\mathcal{N}^{p}:=\left\{X \in L^{0}(\Omega): \mathbb{E}\left[|X|^{p}\right]=0\right\}$;

- $\mathcal{N}:=\left\{X \in L^{0}(\Omega): X=0\right.$, c-q.s. $\}$.

It is seen that $\mathcal{L}^{p}$ and $\mathcal{N}^{p}$ are linear spaces and $\mathcal{N}^{p}=\mathcal{N}$, for each $p>0$.

We denote $\mathbb{L}^{p}:=\mathcal{L}^{p} / \mathcal{N}$. As usual, we do not take care about the distinction between classes and their representatives.

Lemma 1.13 Let $X \in \mathbb{L}^{p}$. Then for each $\alpha>0$

$$
c(\{|X|>\alpha\}) \leq \frac{\mathbb{E}\left[|X|^{p}\right]}{\alpha^{p}} .
$$

Proof. Just apply Markov inequality under each $P \in \mathcal{P}$.

Similar to the classical results, we get the following proposition and the proof is omitted which is similar to the classical arguments.

Proposition 1.14 We have

1. For each $p \geq 1, \mathbb{L}^{p}$ is a Banach space under the norm $\|X\|_{p}:=\left(\mathbb{E}\left[|X|^{p}\right]\right)^{\frac{1}{p}}$. 
2. For each $p<1, \mathbb{L}^{p}$ is a complete metric space under the distance $d(X, Y):=\mathbb{E}\left[|X-Y|^{p}\right]$.

We set

$$
\begin{aligned}
& \mathcal{L}^{\infty}:=\left\{X \in L^{0}(\Omega): \exists \text { a constant } M, \text { s.t. }|X| \leq M, \text { q.s. }\right\} \\
& \mathbb{L}^{\infty}:=\mathcal{L}^{\infty} / \mathcal{N} .
\end{aligned}
$$

Proposition 1.15 Under the norm

$$
\|X\|_{\infty}:=\inf \{M \geq 0:|X| \leq M, \quad \text { q.s. }\}
$$

$\mathbb{L}^{\infty}$ is a Banach space.

Proof. From $\left\{|X|>\|X\|_{\infty}\right\}=\cup_{n=1}^{\infty}\left\{|X| \geq\|X\|_{\infty}+\frac{1}{n}\right\}$ we know that $|X| \leq$ $\|X\|_{\infty}$, q.s., then it is easy to check that $\|\cdot\|_{\infty}$ is a norm. The proof of the completeness of $\mathbb{L}^{\infty}$ is similar to the classical result.

With respect to the distance defined on $\mathbb{L}^{p}, p>0$, we denote by

- $\mathbb{L}_{b}^{p}$ the completion of $B_{b}(\Omega)$.

- $\mathbb{L}_{c}^{p}$ the completion of $C_{b}(\Omega)$.

By Proposition 1.14, we have

$$
\mathbb{L}_{c}^{p} \subset \mathbb{L}_{b}^{p} \subset \mathbb{L}^{p}, \quad p>0 .
$$

The following Proposition is obvious and the proof is left to the reader.

Proposition 1.16 We have

1. Let $p, q>1, \frac{1}{p}+\frac{1}{q}=1$. Then $X \in \mathbb{L}^{p}$ and $Y \in \mathbb{L}^{q}$ implies

$$
X Y \in \mathbb{L}^{1} \text { and } \mathbb{E}[|X Y|] \leq\left(\mathbb{E}\left[|X|^{p}\right]\right)^{\frac{1}{p}}\left(\mathbb{E}\left[|Y|^{q}\right]\right)^{\frac{1}{q}} ;
$$

Moreover $X \in \mathbb{L}_{c}^{p}$ and $Y \in \mathbb{L}_{c}^{q}$ implies $X Y \in \mathbb{L}_{c}^{1}$;

2. $\mathbb{L}^{p_{1}} \subset \mathbb{L}^{p_{2}}, \mathbb{L}_{b}^{p_{1}} \subset \mathbb{L}_{b}^{p_{2}}, \mathbb{L}_{c}^{p_{1}} \subset \mathbb{L}_{c}^{p_{2}}, 0<p_{2} \leq p_{1} \leq \infty$;

3. $\|X\|_{p} \uparrow\|X\|_{\infty}$, for each $X \in \mathbb{L}^{\infty}$.

Proposition 1.17 Let $p \in(0, \infty]$ and $\left(X_{n}\right)$ be a sequence in $\mathbb{L}^{p}$ which converges to $X$ in $\mathbb{L}^{p}$. Then there exists a subsequence $\left(X_{n_{k}}\right)$ which converges to $X$ quasisurely in the sense that it converges to $X$ outside a polar set. 
Proof. Let us assume $p \in(0, \infty)$, the case $p=\infty$ is obvious since the convergence in $\mathbb{L}^{\infty}$ implies the convergence in $\mathbb{L}^{p}$ for all $p$.

One can extract a subsequence $\left(X_{n_{k}}\right)$ such that

$$
\mathbb{E}\left[\left|X-X_{n_{k}}\right|^{p}\right] \leq 1 / k^{p+2}, \quad k \in \mathbb{N} .
$$

We set for all $k$

$$
A_{k}=\left\{\left|X-X_{n_{k}}\right|>1 / k\right\},
$$

then as a consequence of the Markov property (Lemma 1.13) and the BorelCantelli Lemma 1.5. $c\left(\overline{\lim }_{k \rightarrow \infty} A_{k}\right)=0$. As it is clear that on $\left(\overline{\lim }_{k \rightarrow \infty} A_{k}\right)^{c}$, $\left(X_{n_{k}}\right)$ converges to $X$, the proposition is proved.

We now give a description of $\mathbb{L}_{b}^{p}$.

Proposition 1.18 For each $p>0$,

$$
\mathbb{L}_{b}^{p}=\left\{X \in \mathbb{L}^{p}: \lim _{n \rightarrow \infty} \mathbb{E}\left[|X|^{p} \mathbf{1}_{\{|X|>n\}}\right]=0\right\} .
$$

Proof. We denote $J_{p}=\left\{X \in \mathbb{L}^{p}: \lim _{n \rightarrow \infty} \mathbb{E}\left[|X|^{p} \mathbf{1}_{\{|X|>n\}}\right]=0\right\}$. For each $X \in J_{p}$ let $X_{n}=(X \wedge n) \vee(-n) \in B_{b}(\Omega)$. We have

$$
\mathbb{E}\left[\left|X-X_{n}\right|^{p}\right] \leq \mathbb{E}\left[|X|^{p} \mathbf{1}_{\{|X|>n\}}\right] \rightarrow 0, \text { as } n \rightarrow \infty .
$$

Thus $X \in \mathbb{L}_{b}^{p}$.

On the other hand, for each $X \in \mathbb{L}_{b}^{p}$, we can find a sequence $\left\{Y_{n}\right\}_{n=1}^{\infty}$ in $B_{b}(\Omega)$ such that $\mathbb{E}\left[\left|X-Y_{n}\right|^{p}\right] \rightarrow 0$. Let $y_{n}=\sup _{\omega \in \Omega}\left|Y_{n}(\omega)\right|$ and $X_{n}=\left(X \wedge y_{n}\right) \vee\left(-y_{n}\right)$. Since $\left|X-X_{n}\right| \leq\left|X-Y_{n}\right|$, we have $\mathbb{E}\left[\left|X-X_{n}\right|^{p}\right] \rightarrow 0$. This clearly implies that for any sequence $\left(\alpha_{n}\right)$ tending to $\infty, \lim _{n \rightarrow \infty} \mathbb{E}\left[\left|X-\left(X \wedge \alpha_{n}\right) \vee\left(-\alpha_{n}\right)\right|^{p}\right]=0$. Now we have, for all $n \in \mathbb{N}$,

$$
\begin{aligned}
\mathbb{E}\left[|X|^{p} \mathbf{1}_{\{|X|>n\}}\right] & =\mathbb{E}\left[(|X|-n+n)^{p} \mathbf{1}_{\{|X|>n\}}\right] \\
& \leq\left(1 \vee 2^{p-1}\right)\left(\mathbb{E}\left[(|X|-n)^{p} \mathbf{1}_{\{|X|>n\}}\right]+n^{p} c(|X|>n)\right) .
\end{aligned}
$$

The first term of the right hand side tends to 0 since

$$
\mathbb{E}\left[(|X|-n)^{p} \mathbf{1}_{\{|X|>n\}}\right]=\mathbb{E}\left[|X-(X \wedge n) \vee(-n)|^{p}\right] \rightarrow 0 .
$$

For the second term, since

$$
\frac{n^{p}}{2^{p}} \mathbf{1}_{\{|X|>n\}} \leq\left(|X|-\frac{n}{2}\right)^{p} \mathbf{1}_{\{|X|>n\}} \leq\left(|X|-\frac{n}{2}\right)^{p} \mathbf{1}_{\left\{|X|>\frac{n}{2}\right\}}
$$

we have

$$
\frac{n^{p}}{2^{p}} c(|X|>n)=\frac{n^{p}}{2^{p}} \mathbb{E}\left[\mathbf{1}_{\{|X|>n\}}\right] \leq \mathbb{E}\left[\left(|X|-\frac{n}{2}\right)^{p} \mathbf{1}_{\left\{|X|>\frac{n}{2}\right\}}\right] \rightarrow 0 .
$$

Consequently $X \in J_{p}$.

Proposition 1.19 Let $X \in \mathbb{L}_{b}^{1}$. Then for each $\varepsilon>0$, there exists a $\delta>0$, such that for all $A \in \mathcal{B}(\Omega)$ with $c(A) \leq \delta$, we have $\mathbb{E}\left[|X| \mathbf{1}_{A}\right] \leq \varepsilon$. 
Proof. For each $\varepsilon>0$, by Proposition 1.18 there exists an $N>0$ such that $\mathbb{E}\left[|X| \mathbf{1}_{\{|X|>N\}}\right] \leq \frac{\varepsilon}{2}$. Take $\delta=\frac{\varepsilon}{2 N}$. Then for a subset $A \in \mathcal{B}(\Omega)$ with $c(A) \leq \delta$, we have

$$
\begin{aligned}
\mathbb{E}\left[|X| \mathbf{1}_{A}\right] & \leq \mathbb{E}\left[|X| \mathbf{1}_{A} \mathbf{1}_{\{|X|>N\}}\right]+\mathbb{E}\left[|X| \mathbf{1}_{A} \mathbf{1}_{\{|X| \leq N\}}\right] \\
& \leq \mathbb{E}\left[|X| \mathbf{1}_{\{|X|>N\}}\right]+N c(A) \leq \varepsilon
\end{aligned}
$$

It is important to note that not every element in $\mathbb{L}^{p}$ satisfies the condition $\lim _{n \rightarrow \infty} \mathbb{E}\left[|X|^{p} \mathbf{1}_{\{|X|>n\}}\right]=0$. We give the following two counterexamples to show that $\mathbb{L}^{1}$ and $\mathbb{L}_{b}^{1}$ are different spaces even under the case that $\mathcal{P}$ is weakly compact.

Example 1.20 Let $\Omega=\mathbb{N}, \mathcal{P}=\left\{P_{n}: n \in \mathbb{N}\right\}$ where $P_{1}(\{1\})=1$ and $P_{n}(\{1\})=1-\frac{1}{n}, P_{n}(\{n\})=\frac{1}{n}$, for $n=2,3, \cdots$. $\mathcal{P}$ is weakly compact. We consider a function $X$ on $\mathbb{N}$ defined by $X(n)=n, n \in \mathbb{N}$. We have $\mathbb{E}[|X|]=2$ but $\mathbb{E}\left[|X| \mathbf{1}_{\{|X|>n\}}\right]=1 \not \rightarrow 0$. In this case, $X \in \mathbb{L}^{1}$ but $X \notin \mathbb{L}_{b}^{1}$.

Example 1.21 Let $\Omega=\mathbb{N}, \mathcal{P}=\left\{P_{n}: n \in \mathbb{N}\right\}$ where $P_{1}(\{1\})=1$ and $P_{n}(\{1\})=1-\frac{1}{n^{2}}, P_{n}(\{k n\})=\frac{1}{n^{3}}, k=1,2, \ldots, n$, for $n=2,3, \cdots$. $\mathcal{P}$ is weakly compact. We consider a function $X$ on $\mathbb{N}$ defined by $X(n)=n, n \in \mathbb{N}$. We have $\mathbb{E}[|X|]=\frac{25}{16}$ and $n \mathbb{E}\left[\mathbf{1}_{\{|X| \geq n\}}\right]=\frac{1}{n} \rightarrow 0$, but $\mathbb{E}\left[|X| \mathbf{1}_{\{|X| \geq n\}}\right]=\frac{1}{2}+\frac{1}{2 n} \not \rightarrow 0$. In this case, $X$ is in $\mathbb{L}^{1}$, continuous and $n \mathbb{E}\left[\mathbf{1}_{\{|X| \geq n\}}\right] \rightarrow 0$, but it is not in $\mathbb{L}_{b}^{1}$.

\subsection{Properties of elements in $\mathbb{L}_{c}^{p}$}

Definition 1.22 A mapping $X$ on $\Omega$ with values in a topological space is said to be quasi-continuous (q.c.) if

$\forall \varepsilon>0$, there exists an open set $O$ with $c(O)<\varepsilon$ such that $\left.X\right|_{O^{c}}$ is continuous.

Definition 1.23 We say that $X: \Omega \rightarrow \mathbb{R}$ has a quasi-continuous version if there exists a quasi-continuous function $Y: \Omega \rightarrow \mathbb{R}$ with $X=Y$ q.s..

Proposition 1.24 Let $p>0$. Then each element in $\mathbb{L}_{c}^{p}$ has a quasi-continuous version.

Proof. Let $\left(X_{n}\right)$ be a Cauchy sequence in $C_{b}(\Omega)$ for the distance on $\mathbb{L}^{p}$. Let us choose a subsequence $\left(X_{n_{k}}\right)_{k \geq 1}$ such that

$$
\mathbb{E}\left[\left|X_{n_{k+1}}-X_{n_{k}}\right|^{p}\right] \leq 2^{-2 k}, \quad \forall k \geq 1,
$$

and set for all $k$,

$$
A_{k}=\bigcup_{i=k}^{\infty}\left\{\left|X_{n_{i+1}}-X_{n_{i}}\right|>2^{-i / p}\right\}
$$

Thanks to the subadditivity property and the Markov inequality, we have

$$
c\left(A_{k}\right) \leq \sum_{i=k}^{\infty} c\left(\left|X_{n_{i+1}}-X_{n_{i}}\right|>2^{-i / p}\right) \leq \sum_{i=k}^{\infty} 2^{-i}=2^{-k+1} .
$$


As a consequence, $\lim _{k \rightarrow \infty} c\left(A_{k}\right)=0$, so the Borel set $A=\bigcap_{k=1}^{\infty} A_{k}$ is polar. As each $X_{n_{k}}$ is continuous, for all $k \geq 1, A_{k}$ is an open set. Moreover, for all $k,\left(X_{n_{i}}\right)$ converges uniformly on $A_{k}^{c}$ so that the limit is continuous on each $A_{k}^{c}$. This yields the result.

The following theorem gives a concrete characterization of the space $\mathbb{L}_{c}^{p}$.

Theorem 1.25 For each $p>0$,

$\mathbb{L}_{c}^{p}=\left\{X \in \mathbb{L}^{p}: X\right.$ has a quasi-continuous version, $\left.\lim _{n \rightarrow \infty} \mathbb{E}\left[|X|^{p} \mathbf{1}_{\{|X|>n\}}\right]=0\right\}$.

Proof. We denote

$J_{p}=\left\{X \in \mathbb{L}^{p}: X\right.$ has a quasi-continuous version, $\left.\lim _{n \rightarrow \infty} \mathbb{E}\left[|X|^{p} \mathbf{1}_{\{|X|>n\}}\right]=0\right\}$.

Let $X \in \mathbb{L}_{c}^{p}$, we know by Proposition 1.24 that $X$ has a quasi-continuous version. Since $X \in \mathbb{L}_{b}^{p}$, we have by Proposition 1.18 that $\lim _{n \rightarrow \infty} \mathbb{E}\left[|X|^{p} \mathbf{1}_{\{|X|>n\}}\right]=0$. Thus $X \in J_{p}$.

On the other hand, let $X \in J_{p}$ be quasi-continuous. Define $Y_{n}=(X \wedge n) \vee(-n)$ for all $n \in \mathbb{N}$. As $\mathbb{E}\left[|X|^{p} \mathbf{1}_{\{|X|>n\}}\right] \rightarrow 0$, we have $\mathbb{E}\left[\left|X-Y_{n}\right|^{p}\right] \rightarrow 0$.

Moreover, for all $n \in \mathbb{N}$, as $Y_{n}$ is quasi-continuous, there exists a closed set $F_{n}$ such that $c\left(F_{n}^{c}\right)<\frac{1}{n^{p+1}}$ and $Y_{n}$ is continuous on $F_{n}$. It follows from Tietze's extension theorem that there exists $Z_{n} \in C_{b}(\Omega)$ such that

$$
\left|Z_{n}\right| \leq n \text { and } Z_{n}=Y_{n} \text { on } F_{n} .
$$

We then have

$$
\mathbb{E}\left[\left|Y_{n}-Z_{n}\right|^{p}\right] \leq(2 n)^{p} c\left(F_{n}^{c}\right) \leq \frac{(2 n)^{p}}{n^{p+1}} .
$$

So $\mathbb{E}\left[\left|X-Z_{n}\right|^{p}\right] \leq\left(1 \vee 2^{p-1}\right)\left(\mathbb{E}\left[\left|X-Y_{n}\right|^{p}\right]+\mathbb{E}\left[\left|Y_{n}-Z_{n}\right|^{p}\right]\right) \rightarrow 0$, and $X \in \mathbb{L}_{c}^{p}$.

We give the following example to show that $\mathbb{L}_{c}^{p}$ is different from $\mathbb{L}_{b}^{p}$ even under the case that $\mathcal{P}$ is weakly compact.

Example 1.26 Let $\Omega=[0,1], \mathcal{P}=\left\{\delta_{x}: x \in[0,1]\right\}$ is weakly compact. It is seen that $\mathbb{L}_{c}^{p}=C_{b}(\Omega)$ which is different from $\mathbb{L}_{b}^{p}$.

We denote $\mathbb{L}_{c}^{\infty}:=\left\{X \in \mathbb{L}^{\infty}: X\right.$ has a quasi-continuous version $\}$, we have

Proposition 1.27 $\mathbb{L}_{c}^{\infty}$ is a closed linear subspace of $\mathbb{L}^{\infty}$.

Proof. For each Cauchy sequence $\left\{X_{n}\right\}_{n=1}^{\infty}$ of $\mathbb{L}_{c}^{\infty}$ under $\|\cdot\|_{\infty}$, we can find a subsequence $\left\{X_{n_{i}}\right\}_{i=1}^{\infty}$ such that $\left\|X_{n_{i+1}}-X_{n_{i}}\right\|_{\infty} \leq 2^{-i}$. We may further assume that each $X_{n}$ is quasi-continuous. Then it is easy to prove that for each $\varepsilon>0$, there exists an open set $G$ such that $c(G)<\varepsilon$ and $\left|X_{n_{i+1}}-X_{n_{i}}\right| \leq 2^{-i}$ for all $i \geq 1$ on $G^{c}$, which implies that the limit belongs to $\mathbb{L}_{c}^{\infty}$.

As an application of Theorem 1.25, we can easily get the following results. 
Proposition 1.28 Assume that $X: \Omega \rightarrow \mathbb{R}$ has a quasi-continuous version and that there exists a function $f: \mathbb{R}^{+} \rightarrow \mathbb{R}^{+}$satisfying $\lim _{t \rightarrow \infty} \frac{f(t)}{t^{p}}=\infty$ and $\mathbb{E}[f(|X|)]<\infty$. Then $X \in \mathbb{L}_{c}^{p}$.

Proof. For each $\varepsilon>0$, there exists an $N>0$ such that $\frac{f(t)}{t^{p}} \geq \frac{1}{\varepsilon}$, for all $t \geq N$. Thus

$$
\mathbb{E}\left[|X|^{p} \mathbf{1}_{\{|X|>N\}}\right] \leq \varepsilon \mathbb{E}\left[f(|X|) \mathbf{1}_{\{|X|>N\}}\right] \leq \varepsilon \mathbb{E}[f(|X|)] .
$$

Hence $\lim _{N \rightarrow \infty} \mathbb{E}\left[|X|^{p} \mathbf{1}_{\{|X|>N\}}\right]=0$. From Theorem 1.25 we infer $X \in \mathbb{L}_{c}^{p}$.

Lemma 1.29 Let $\left\{P_{n}\right\}_{n=1}^{\infty} \subset \mathcal{P}$ converge weakly to $P \in \mathcal{P}$. Then for each $X \in \mathbb{L}_{c}^{1}$, we have $E_{P_{n}}[X] \rightarrow E_{P}[X]$.

Proof. We may assume that $X$ is quasi-continuous, otherwise we can consider its quasi-continuous version which does not change the value $E_{Q}$ for each $Q \in \mathcal{P}$. For each $\varepsilon>0$, there exists an $N>0$ such that $\mathbb{E}\left[|X| \mathbf{1}_{\{|X|>N\}}\right]<\frac{\varepsilon}{2}$. Set $X_{N}=(X \wedge N) \vee(-N)$. We can find an open subset $G$ such that $c(G)<\frac{\varepsilon}{4 N}$ and $X_{N}$ is continuous on $G^{c}$. By Tietze's extension theorem, there exists $Y \in C_{b}(\Omega)$ such that $|Y| \leq N$ and $Y=X_{N}$ on $G^{c}$. Obviously, for each $Q \in \mathcal{P}$,

$$
\begin{aligned}
\left|E_{Q}[X]-E_{Q}[Y]\right| & \leq E_{Q}\left[\left|X-X_{N}\right|\right]+E_{Q}\left[\left|X_{N}-Y\right|\right] \\
& \leq \frac{\varepsilon}{2}+2 N \frac{\varepsilon}{4 N}=\varepsilon .
\end{aligned}
$$

It then follows that

$$
\limsup _{n \rightarrow \infty} E_{P_{n}}[X] \leq \lim _{n \rightarrow \infty} E_{P_{n}}[Y]+\varepsilon=E_{P}[Y]+\varepsilon \leq E_{P}[X]+2 \varepsilon,
$$

and similarly $\liminf _{n \rightarrow \infty} E_{P_{n}}[X] \geq E_{P}[X]-2 \varepsilon$. Since $\varepsilon$ can be arbitrarily small, we then have $E_{P_{n}}[X] \rightarrow E_{P}[X]$.

Remark 1.30 For continuous $X$, the above lemma is Lemma 3.8 .7 in [15].

Now we give an extension of Theorem 1.12

Theorem 1.31 Let $\mathcal{P}$ be weakly compact and let $\left\{X_{n}\right\}_{n=1}^{\infty} \subset \mathbb{L}_{c}^{1}$ be such that $X_{n} \downarrow X$, q.s.. Then $\mathbb{E}\left[X_{n}\right] \downarrow \mathbb{E}[X]$.

Remark 1.32 It is important to note that $X$ does not necessarily belong to $\mathbb{L}_{c}^{1}$.

Proof. For the case $\mathbb{E}[X]>-\infty$, if there exists a $\delta>0$ such that $\mathbb{E}\left[X_{n}\right]>$ $\mathbb{E}[X]+\delta, n=1,2, \cdots$, we then can find a $P_{n} \in \mathcal{P}$ such that $E_{P_{n}}\left[X_{n}\right]>$ $\mathbb{E}[X]+\delta-\frac{1}{n}, n=1,2, \cdots$. Since $\mathcal{P}$ is weakly compact, we then can find a subsequence $\left\{P_{n_{i}}\right\}_{i=1}^{\infty}$ that converges weakly to some $P \in \mathcal{P}$. From which it follows that

$$
\begin{aligned}
E_{P}\left[X_{n_{i}}\right] & =\lim _{j \rightarrow \infty} E_{P_{n_{j}}}\left[X_{n_{i}}\right] \geq \limsup _{j \rightarrow \infty} E_{P_{n_{j}}}\left[X_{n_{j}}\right] \\
& \geq \limsup _{j \rightarrow \infty}\left\{\mathbb{E}[X]+\delta-\frac{1}{n_{j}}\right\}=\mathbb{E}[X]+\delta, \quad i=1,2, \cdots .
\end{aligned}
$$


Thus $E_{P}[X] \geq \mathbb{E}[X]+\delta$. This contradicts the definition of $\mathbb{E}[\cdot]$. The proof for the case $\mathbb{E}[X]=-\infty$ is analogous.

We immediately have the following corollary.

Corollary 1.33 Let $\mathcal{P}$ be weakly compact and let $\left\{X_{n}\right\}_{n=1}^{\infty}$ be a sequence in $\mathbb{L}_{c}^{1}$ decreasingly converging to 0 q.s.. Then $\mathbb{E}\left[X_{n}\right] \downarrow 0$.

\subsection{Kolmogorov's criterion}

Definition 1.34 Let I be a set of indices, $\left(X_{t}\right)_{t \in I}$ and $\left(Y_{t}\right)_{t \in I}$ be two processes indexed by $I$. We say that $Y$ is a quasi-modification of $X$ if for all $t \in I$, $X_{t}=Y_{t}$ q.s..

Remark 1.35 In the above definition, quasi-modification is also called modification in some papers.

We now give a Kolmogorov criterion for a process indexed by $\mathbb{R}^{d}$ with $d \in \mathbb{N}$.

Theorem 1.36 Let $p>0$ and $\left(X_{t}\right)_{t \in[0,1]^{d}}$ be a process such that for all $t \in$ $[0,1]^{d}, X_{t}$ belongs to $\mathbb{L}^{p}$. Assume that there exist positive constants $c$ and $\varepsilon$ such that

$$
\mathbb{E}\left[\left|X_{t}-X_{s}\right|^{p}\right] \leq c|t-s|^{d+\varepsilon}
$$

Then $X$ admits a modification $\tilde{X}$ such that

$$
\mathbb{E}\left[\left(\sup _{s \neq t} \frac{\left|\tilde{X}_{t}-\tilde{X}_{s}\right|}{|t-s|^{\alpha}}\right)^{p}\right]<\infty
$$

for every $\alpha \in[0, \varepsilon / p)$. As a consequence, paths of $\tilde{X}$ are quasi-surely Höder continuous of order $\alpha$ for every $\alpha<\varepsilon / p$ in the sense that there exists a Borel set $N$ of capacity 0 such that for all $w \in N^{c}$, the map $t \rightarrow \tilde{X}(w)$ is Höder continuous of order $\alpha$ for every $\alpha<\varepsilon / p$. Moreover, if $X_{t} \in \mathbb{L}_{c}^{p}$ for each $t$, then we also have $\tilde{X}_{t} \in \mathbb{L}_{c}^{p}$.

Proof. Let $D$ be the set of dyadic points in $[0,1]^{d}$ :

$$
D=\left\{\left(\frac{i_{1}}{2^{n}}, \cdots, \frac{i_{d}}{2^{n}}\right) ; n \in \mathbb{N}, i_{1}, \cdots, i_{d} \in\left\{0,1, \cdots, 2^{n}\right\}\right\} .
$$

Let $\alpha \in[0, \varepsilon / p)$. We set

$$
M=\sup _{s, t \in D, s \neq t} \frac{\left|X_{t}-X_{s}\right|}{|t-s|^{\alpha}} .
$$

Thanks to the classical Kolmogorov's criterion (see Revuz-Yor [11]), we know that for any $P \in \mathcal{P}, E_{P}\left[M^{p}\right]$ is finite and uniformly bounded with respect to $P$ so that

$$
\mathbb{E}\left[M^{p}\right]=\sup _{P \in \mathcal{P}} E_{P}\left[M^{p}\right]<\infty .
$$


As a consequence, the map $t \rightarrow X_{t}$ is uniformly continuous on $D$ quasi-surely and so we can define

$$
\forall t \in[0,1]^{d}, \tilde{X}_{t}=\lim _{s \rightarrow t, s \in D} X_{s}
$$

It is now clear that $\tilde{X}$ satisfies the enounced properties.

\section{$\S 2 \quad G$-expectation as an Upper Expectation}

In the following sections of this Chapter, let $\Omega=C_{0}^{d}\left(\mathbb{R}^{+}\right)$denote the space of all $\mathbb{R}^{d}$-valued continuous functions $\left(\omega_{t}\right)_{t \in \mathbb{R}^{+}}$, with $\omega_{0}=0$, equipped with the distance

$$
\rho\left(\omega^{1}, \omega^{2}\right):=\sum_{i=1}^{\infty} 2^{-i}\left[\left(\max _{t \in[0, i]}\left|\omega_{t}^{1}-\omega_{t}^{2}\right|\right) \wedge 1\right],
$$

and let $\bar{\Omega}=\left(\mathbb{R}^{d}\right)^{[0, \infty)}$ denote the space of all $\mathbb{R}^{d}$-valued functions $\left(\bar{\omega}_{t}\right)_{t \in \mathbb{R}^{+}}$. Let $\mathcal{B}(\Omega)$ denote the $\sigma$-algebra generated by all open sets and let $\mathcal{B}(\bar{\Omega})$ denote the $\sigma$-algebra generated by all finite dimensional cylinder sets. The corresponding canonical process is $B_{t}(\omega)=\omega_{t}$ (respectively, $\left.\bar{B}_{t}(\bar{\omega})=\bar{\omega}_{t}\right), t \in[0, \infty)$ for $\omega \in \Omega$ (respectively, $\bar{\omega} \in \bar{\Omega}$ ). The spaces of Lipschitzian cylinder functions on $\Omega$ and $\bar{\Omega}$ are denoted respectively by

$L_{i p}(\Omega):=\left\{\varphi\left(B_{t_{1}}, B_{t_{2}}, \cdots, B_{t_{n}}\right): \forall n \geq 1, t_{1}, \cdots, t_{n} \in[0, \infty), \forall \varphi \in C_{l . L i p}\left(\mathbb{R}^{d \times n}\right)\right\}$,

$L_{i p}(\bar{\Omega}):=\left\{\varphi\left(\bar{B}_{t_{1}}, \bar{B}_{t_{2}}, \cdots, \bar{B}_{t_{n}}\right): \forall n \geq 1, t_{1}, \cdots, t_{n} \in[0, \infty), \forall \varphi \in C_{l . L i p}\left(\mathbb{R}^{d \times n}\right)\right\}$.

Let $G(\cdot): \mathbb{S}(d) \rightarrow \mathbb{R}$ be a given continuous monotonic and sublinear function. Following Sec.2 in Chap III, we can construct the corresponding $G$-expectation $\hat{\mathbb{E}}$ on $\left(\Omega, L_{i p}(\Omega)\right)$. Due to the natural correspondence of $L_{i p}(\bar{\Omega})$ and $L_{i p}(\Omega)$, we also construct a sublinear expectation $\overline{\mathbb{E}}$ on $\left(\bar{\Omega}, L_{i p}(\bar{\Omega})\right)$ such that $\left(\bar{B}_{t}(\bar{\omega})\right)_{t \geq 0}$ is a $G$-Brownian motion.

The main objective of this section is to find a weakly compact family of $(\sigma$ additive) probability measures on $(\Omega, \mathcal{B}(\Omega))$ to represent $G$-expectation $\hat{\mathbb{E}}$. The following lemmas are a variety of Lemma 3.3 and 3.4

Lemma 2.1 Let $0 \leq t_{1}<t_{2}<\cdots<t_{m}<\infty$ and $\left\{\varphi_{n}\right\}_{n=1}^{\infty} \subset C_{\text {l.Lip }}\left(\mathbb{R}^{d \times m}\right)$ satisfy $\varphi_{n} \downarrow 0$. Then $\overline{\mathbb{E}}\left[\varphi_{n}\left(\bar{B}_{t_{1}}, \bar{B}_{t_{2}}, \cdots, \bar{B}_{t_{m}}\right)\right] \downarrow 0$.

We denote $\mathcal{T}:=\left\{\underline{t}=\left(t_{1}, \ldots, t_{m}\right): \forall m \in \mathbb{N}, 0 \leq t_{1}<t_{2}<\cdots<t_{m}<\infty\right\}$.

Lemma 2.2 Let $E$ be a finitely additive linear expectation dominated by $\overline{\mathbb{E}}$ on $L_{i p}(\bar{\Omega})$. Then there exists a unique probability measure $Q$ on $(\bar{\Omega}, \mathcal{B}(\bar{\Omega}))$ such that $E[X]=E_{Q}[X]$ for each $X \in L_{i p}(\bar{\Omega})$.

Proof. For each fixed $\underline{t}=\left(t_{1}, \ldots, t_{m}\right) \in \mathcal{T}$, by Lemma 2.1. for each sequence $\left\{\varphi_{n}\right\}_{n=1}^{\infty} \subset C_{l . L i p}\left(\mathbb{R}^{d \times m}\right)$ satisfying $\varphi_{n} \downarrow 0$, we have $E\left[\varphi_{n}\left(\bar{B}_{t_{1}}, \bar{B}_{t_{2}}, \cdots, \bar{B}_{t_{m}}\right)\right] \downarrow$ 
0. By Daniell-Stone's theorem (see Appendix B), there exists a unique probability measure $Q_{\underline{\underline{t}}}$ on $\left(\mathbb{R}^{d \times m}, \mathcal{B}\left(\mathbb{R}^{d \times m}\right)\right)$ such that $E_{Q_{\underline{t}}}[\varphi]=E\left[\varphi\left(\bar{B}_{t_{1}}, \bar{B}_{t_{2}}, \cdots, \bar{B}_{t_{m}}\right)\right]$ for each $\varphi \in C_{l . L i p}\left(\mathbb{R}^{d \times m}\right)$. Thus we get a family of finite dimensional distributions $\left\{Q_{\underline{t}}: \underline{t} \in \mathcal{T}\right\}$. It is easy to check that $\left\{Q_{\underline{t}}: \underline{t} \in \mathcal{T}\right\}$ is consistent. Then by Kolmogorov's consistent theorem, there exists a probability measure $Q$ on $(\bar{\Omega}, \mathcal{B}(\bar{\Omega}))$ such that $\left\{Q_{\underline{t}}: \underline{t} \in \mathcal{T}\right\}$ is the finite dimensional distributions of $Q$. Assume that there exists another probability measure $\bar{Q}$ satisfying the condition, by Daniell-Stone's theorem, $Q$ and $\bar{Q}$ have the same finite-dimensional distributions. Then by monotone class theorem, $Q=\bar{Q}$. The proof is complete.

Lemma 2.3 There exists a family of probability measures $\mathcal{P}_{e}$ on $(\bar{\Omega}, \mathcal{B}(\bar{\Omega}))$ such that

$$
\overline{\mathbb{E}}[X]=\max _{Q \in \mathcal{P}_{e}} E_{Q}[X], \quad \text { for } X \in L_{i p}(\bar{\Omega}) .
$$

Proof. By the representation theorem of sublinear expectation and Lemma 2.2. it is easy to get the result.

For this $\mathcal{P}_{e}$, we define the associated capacity:

$$
\tilde{c}(A):=\sup _{Q \in \mathcal{P}_{e}} Q(A), \quad A \in \mathcal{B}(\bar{\Omega}),
$$

and the upper expectation for each $\mathcal{B}(\bar{\Omega})$-measurable real function $X$ which makes the following definition meaningful:

$$
\tilde{\mathbb{E}}[X]:=\sup _{Q \in \mathcal{P}_{e}} E_{Q}[X]
$$

Theorem 2.4 For $(\bar{B})_{t \geq 0}$, there exists a continuous modification $(\tilde{B})_{t \geq 0}$ of $\bar{B}$ (i.e., $\tilde{c}\left(\left\{\tilde{B}_{t} \neq \bar{B}_{t}\right\}\right)=0$, for each $\left.t \geq 0\right)$ such that $\tilde{B}_{0}=0$.

Proof. By Lemma 2.3. we know that $\overline{\mathbb{E}}=\tilde{\mathbb{E}}$ on $L_{i p}(\bar{\Omega})$. On the other hand, we have

$$
\tilde{\mathbb{E}}\left[\left|\bar{B}_{t}-\bar{B}_{s}\right|^{4}\right]=\overline{\mathbb{E}}\left[\left|\bar{B}_{t}-\bar{B}_{s}\right|^{4}\right]=d|t-s|^{2} \text { for } s, t \in[0, \infty),
$$

where $d$ is a constant depending only on $G$. By Theorem 1.36, there exists a continuous modification $\tilde{B}$ of $\bar{B}$. Since $\tilde{c}\left(\left\{\bar{B}_{0} \neq 0\right\}\right)=0$, we can set $\tilde{B}_{0}=0$. The proof is complete.

For each $Q \in \mathcal{P}_{e}$, let $Q \circ \tilde{B}^{-1}$ denote the probability measure on $(\Omega, \mathcal{B}(\Omega))$ induced by $\tilde{B}$ with respect to $Q$. We denote $\mathcal{P}_{1}=\left\{Q \circ \tilde{B}^{-1}: Q \in \mathcal{P}_{e}\right\}$. By Lemma 2.4 we get

$$
\tilde{\mathbb{E}}\left[\left|\tilde{B}_{t}-\tilde{B}_{s}\right|^{4}\right]=\tilde{\mathbb{E}}\left[\left|\bar{B}_{t}-\bar{B}_{s}\right|^{4}\right]=d|t-s|^{2}, \forall s, t \in[0, \infty) .
$$

Applying the well-known result of moment criterion for tightness of KolmogorovChentsov's type (see Appendix B), we conclude that $\mathcal{P}_{1}$ is tight. We denote by $\mathcal{P}=\overline{\mathcal{P}}_{1}$ the closure of $\mathcal{P}_{1}$ under the topology of weak convergence, then $\mathcal{P}$ is weakly compact.

Now, we give the representation of $G$-expectation. 
Theorem 2.5 For each continuous monotonic and sublinear function $G: \mathbb{S}(d) \rightarrow$ $\mathbb{R}$, let $\hat{\mathbb{E}}$ be the corresponding $G$-expectation on $\left(\Omega, L_{i p}(\Omega)\right)$. Then there exists a weakly compact family of probability measures $\mathcal{P}$ on $(\Omega, \mathcal{B}(\Omega)$ ) such that

$$
\hat{\mathbb{E}}[X]=\max _{P \in \mathcal{P}} E_{P}[X] \quad \text { for } X \in L_{i p}(\Omega) .
$$

Proof. By Lemma 2.3 and Lemma 2.4 we have

$$
\hat{\mathbb{E}}[X]=\max _{P \in \mathcal{P}_{1}} E_{P}[X] \quad \text { for } X \in L_{i p}(\Omega) .
$$

For each $X \in L_{i p}(\Omega)$, by Lemma 2.1, we get $\hat{\mathbb{E}}[|X-(X \wedge N) \vee(-N)|] \downarrow 0$ as $N \rightarrow \infty$. Noting that $\mathcal{P}=\overline{\mathcal{P}}_{1}$, by the definition of weak convergence, we get the result.

Remark 2.6 In fact, we can construct the family $\mathcal{P}$ in a more explicit way: Let $\left(W_{t}\right)_{t \geq 0}=\left(W_{t}^{i}\right)_{i=1, t \geq 0}^{d}$ be a d-dimensional Brownian motion in this space. The filtration generated by $W$ is denoted by $\mathcal{F}_{t}^{W}$. Now let $\Gamma$ be the bounded, closed and convex subset in $\mathbb{R}^{d \times d}$ such that

$$
G(A)=\sup _{\gamma \in \Gamma} \operatorname{tr}\left[A \gamma \gamma^{T}\right], \quad A \in \mathbb{S}(d),
$$

(see (1.13) in Ch. II) and $\mathcal{A}_{\Gamma}$ the collection of all $\Theta$-valued $\left(\mathcal{F}_{t}^{W}\right)_{t \geq 0}$-adapted process $[0, \infty)$. We denote

$$
B_{t}^{\gamma}:=\int_{0}^{T} \gamma_{s} d W_{s}, t \geq 0, \quad \gamma \in \mathcal{A}_{\Gamma} .
$$

and $\mathcal{P}_{0}$ the collection of probability measures on the canonical space $(\Omega, \mathcal{B}(\Omega))$ induced by $\left\{B^{\gamma}: \gamma \in \mathcal{A}_{\Gamma}\right\}$. Then $\mathcal{P}=\overline{\mathcal{P}}_{0}$ (see [3\}] for details).

\section{$\S 3 \quad G$-capacity and Paths of $G$-Brownian Motion}

According to Theorem 2.5, we obtain a weakly compact family of probability measures $\mathcal{P}$ on $(\Omega, \mathcal{B}(\Omega))$ to represent $G$-expectation $\hat{\mathbb{E}}[\cdot]$. For this $\mathcal{P}$, we define the associated $G$-capacity:

$$
\hat{c}(A):=\sup _{P \in \mathcal{P}} P(A), \quad A \in \mathcal{B}(\Omega)
$$

and upper expectation for each $X \in L^{0}(\Omega)$ which makes the following definition meaningful:

$$
\overline{\mathbb{E}}[X]:=\sup _{P \in \mathcal{P}} E_{P}[X] .
$$

By Theorem 2.5, we know that $\overline{\mathbb{E}}=\hat{\mathbb{E}}$ on $L_{i p}(\Omega)$, thus the $\hat{\mathbb{E}}[|\cdot|$-completion and the $\overline{\mathbb{E}}[|\cdot|]$-completion of $L_{i p}(\Omega)$ are the same. 
For each $T>0$, we also denote by $\Omega_{T}=C_{0}^{d}([0, T])$ equipped with the distance

$$
\rho\left(\omega^{1}, \omega^{2}\right)=\left\|\omega^{1}-\omega^{2}\right\|_{C_{0}^{d}([0, T])}:=\max _{0 \leq t \leq T}\left|\omega_{t}^{1}-\omega_{t}^{2}\right| .
$$

We now prove that $L_{G}^{1}(\Omega)=\mathbb{L}_{c}^{1}$, where $\mathbb{L}_{c}^{1}$ is defined in Sec.1. First, we need the following classical approximation lemma.

Lemma 3.1 For each $X \in C_{b}(\Omega)$ and $n=1,2, \cdots$, we denote

$$
X^{(n)}(\omega):=\inf _{\omega^{\prime} \in \Omega}\left\{X\left(\omega^{\prime}\right)+n\left\|\omega-\omega^{\prime}\right\|_{C_{0}^{d}([0, n])}\right\} \quad \text { for } \omega \in \Omega .
$$

Then the sequence $\left\{X^{(n)}\right\}_{n=1}^{\infty}$ satisfies:

(i) $-M \leq X^{(n)} \leq X^{(n+1)} \leq \cdots \leq X, M=\sup _{\omega \in \Omega}|X(\omega)|$;

(ii) $\left|X^{(n)}\left(\omega_{1}\right)-X^{(n)}\left(\omega_{2}\right)\right| \leq n\left\|\omega_{1}-\omega_{2}\right\|_{C_{0}^{d}([0, n])}$ for $\omega_{1}, \omega_{2} \in \Omega$;

(iii) $X^{(n)}(\omega) \uparrow X(\omega)$ for $\omega \in \Omega$.

Proof. (i) is obvious.

For (ii), we have

$$
\begin{aligned}
& X^{(n)}\left(\omega_{1}\right)-X^{(n)}\left(\omega_{2}\right) \\
\leq & \sup _{\omega^{\prime} \in \Omega}\left\{\left[X\left(\omega^{\prime}\right)+n\left\|\omega_{1}-\omega^{\prime}\right\|_{C_{0}^{d}([0, n])}\right]-\left[X\left(\omega^{\prime}\right)+n\left\|\omega_{2}-\omega^{\prime}\right\|_{C_{0}^{d}([0, n])}\right]\right\} \\
\leq & n\left\|\omega_{1}-\omega_{2}\right\|_{C_{0}^{d}([0, n])}
\end{aligned}
$$

and, symmetrically, $X^{(n)}\left(\omega_{2}\right)-X^{(n)}\left(\omega_{1}\right) \leq n\left\|\omega_{1}-\omega_{2}\right\|_{C_{0}^{d}([0, n])}$. Thus (ii) follows.

We now prove (iii). For each fixed $\omega \in \Omega$, let $\omega_{n} \in \Omega$ be such that

$$
X\left(\omega_{n}\right)+n\left\|\omega-\omega_{n}\right\|_{C_{0}^{d}([0, n])} \leq X^{(n)}(\omega)+\frac{1}{n} .
$$

It is clear that $n\left\|\omega-\omega_{n}\right\|_{C_{0}^{d}([0, n])} \leq 2 M+1$ or $\left\|\omega-\omega_{n}\right\|_{C_{0}^{d}([0, n])} \leq \frac{2 M+1}{n}$. Since $X \in C_{b}(\Omega)$, we get $X\left(\omega_{n}\right) \rightarrow X(\omega)$ as $n \rightarrow \infty$. We have

$$
X(\omega) \geq X^{(n)}(\omega) \geq X\left(\omega_{n}\right)+n\left\|\omega-\omega_{n}\right\|_{C_{0}^{d}([0, n])}-\frac{1}{n},
$$

thus

$$
n\left\|\omega-\omega_{n}\right\|_{C_{0}^{d}([0, n])} \leq\left|X(\omega)-X\left(\omega_{n}\right)\right|+\frac{1}{n} .
$$

We also have

$$
\begin{aligned}
X\left(\omega_{n}\right)-X(\omega)+n\left\|\omega-\omega_{n}\right\|_{C_{0}^{d}([0, n])} & \geq X^{(n)}(\omega)-X(\omega) \\
& \geq X\left(\omega_{n}\right)-X(\omega)+n\left\|\omega-\omega_{n}\right\|_{C_{0}^{d}([0, n])}-\frac{1}{n} .
\end{aligned}
$$


From the above two relations, we obtain

$$
\begin{aligned}
\left|X^{(n)}(\omega)-X(\omega)\right| & \leq\left|X\left(\omega_{n}\right)-X(\omega)\right|+n\left\|\omega-\omega_{n}\right\|_{C_{0}^{d}([0, n])}+\frac{1}{n} \\
& \leq 2\left(\left|X\left(\omega_{n}\right)-X(\omega)\right|+\frac{1}{n}\right) \rightarrow 0 \text { as } n \rightarrow \infty .
\end{aligned}
$$

Thus (iii) is obtained.

Proposition 3.2 For each $X \in C_{b}(\Omega)$ and $\varepsilon>0$, there exists $Y \in L_{i p}(\Omega)$ such that $\overline{\mathbb{E}}[|Y-X|] \leq \varepsilon$.

Proof. We denote $M=\sup _{\omega \in \Omega}|X(\omega)|$. By Theorem 1.12 and Lemma 3.1. we can find $\mu>0, T>0$ and $\bar{X} \in C_{b}\left(\Omega_{T}\right)$ such that $\overline{\mathbb{E}}[|X-\bar{X}|]<\varepsilon / 3$, $\sup _{\omega \in \Omega}|\bar{X}(\omega)| \leq M$ and

$$
\left|\bar{X}(\omega)-\bar{X}\left(\omega^{\prime}\right)\right| \leq \mu\left\|\omega-\omega^{\prime}\right\|_{C_{0}^{d}([0, T])} \quad \text { for } \omega, \omega^{\prime} \in \Omega .
$$

Now for each positive integer $n$, we introduce a mapping $\omega^{(n)}(\omega): \Omega \rightarrow \Omega$ :

$\omega^{(n)}(\omega)(t)=\sum_{k=0}^{n-1} \frac{\mathbf{1}_{\left[t_{k}^{n}, t_{k+1}^{n}\right)}(t)}{t_{k+1}^{n}-t_{k}^{n}}\left[\left(t_{k+1}^{n}-t\right) \omega\left(t_{k}^{n}\right)+\left(t-t_{k}^{n}\right) \omega\left(t_{k+1}^{n}\right)\right]+\mathbf{1}_{[T, \infty)}(t) \omega(t)$,

where $t_{k}^{n}=\frac{k T}{n}, k=0,1, \cdots, n$. We set $\bar{X}^{(n)}(\omega):=\bar{X}\left(\omega^{(n)}(\omega)\right)$, then

$$
\begin{aligned}
\left|\bar{X}^{(n)}(\omega)-\bar{X}^{(n)}\left(\omega^{\prime}\right)\right| & \leq \mu \sup _{t \in[0, T]}\left|\omega^{(n)}(\omega)(t)-\omega^{(n)}\left(\omega^{\prime}\right)(t)\right| \\
& =\mu \sup _{k \in[0, \cdots, n]}\left|\omega\left(t_{k}^{n}\right)-\omega^{\prime}\left(t_{k}^{n}\right)\right| .
\end{aligned}
$$

We now choose a compact subset $K \subset \Omega$ such that $\overline{\mathbb{E}}\left[\mathbf{1}_{K^{C}}\right] \leq \varepsilon / 6 M$. Since $\sup _{\omega \in K} \sup _{t \in[0, T]}\left|\omega(t)-\omega^{(n)}(\omega)(t)\right| \rightarrow 0$, as $n \rightarrow \infty$, we can choose a sufficiently large $n_{0}$ such that

$$
\begin{aligned}
\sup _{\omega \in K}\left|\bar{X}(\omega)-\bar{X}^{\left(n_{0}\right)}(\omega)\right| & =\sup _{\omega \in K}\left|\bar{X}(\omega)-\bar{X}\left(\omega^{\left(n_{0}\right)}(\omega)\right)\right| \\
& \leq \mu \sup _{\omega \in K} \sup _{t \in[0, T]}\left|\omega(t)-\omega^{\left(n_{0}\right)}(\omega)(t)\right| \\
& <\varepsilon / 3 .
\end{aligned}
$$

Set $Y:=\bar{X}^{\left(n_{0}\right)}$, it follows that

$$
\begin{aligned}
\overline{\mathbb{E}}[|X-Y|] & \leq \overline{\mathbb{E}}[|X-\bar{X}|]+\overline{\mathbb{E}}\left[\left|\bar{X}-\bar{X}^{\left(n_{0}\right)}\right|\right] \\
& \leq \overline{\mathbb{E}}[|X-\bar{X}|]+\overline{\mathbb{E}}\left[\mathbf{1}_{K}\left|\bar{X}-\bar{X}^{\left(n_{0}\right)}\right|\right]+2 M \overline{\mathbb{E}}\left[\mathbf{1}_{K^{C}}\right] \\
& <\varepsilon
\end{aligned}
$$

The proof is complete. 
By Proposition 3.2 we can easily get $L_{G}^{1}(\Omega)=\mathbb{L}_{c}^{1}$. Furthermore, we can get $L_{G}^{p}(\Omega)=\mathbb{L}_{c}^{p}, \forall p>0$.

Thus, we obtain a pathwise description of $L_{G}^{p}(\Omega)$ for each $p>0$ :

$L_{G}^{p}(\Omega)=\left\{X \in L^{0}(\Omega): X\right.$ has a quasi-continuous version and $\left.\lim _{n \rightarrow \infty} \overline{\mathbb{E}}\left[|X|^{p} I_{\{|X|>n\}}\right]=0\right\}$.

Furthermore, $\overline{\mathbb{E}}[X]=\hat{\mathbb{E}}[X]$, for each $X \in L_{G}^{1}(\Omega)$.

Exercise 3.3 Show that, for each $p>0$,

$L_{G}^{p}\left(\Omega_{T}\right)=\left\{X \in L^{0}\left(\Omega_{T}\right): X\right.$ has a quasi-continuous version and $\left.\lim _{n \rightarrow \infty} \overline{\mathbb{E}}\left[|X|^{p} I_{\{|X|>n\}}\right]=0\right\}$.

\section{Notes and Comments}

The results of this chapter for $G$-Brownian motions were mainly obtained by Denis, Hu and Peng (2008) 37] (see also Denis and Martini (2006) 38] and the related comments after Chapter III). Hu and Peng (2009) [58] then have introduced an intrinsic and simple approach. This approach can be regarded as a combination and extension of the original Brownian motion construction approach of Kolmogorov (for more general stochastic processes) and a sort of cylinder Lipschitz functions technique already introduced in Chap. III. Section 1 is from [37] and Theorem [2.5 is firstly obtained in [37], whereas contents of Sections 2 and 3 are mainly from [58].

Choquet capacity was first introduced by Choquet (1953) 24], see also Dellacherie (1972) 32] and the references therein for more properties. The capacitability of Choquet capacity was first studied by Choquet 24] under 2alternating case, see Dellacherie and Meyer (1978 and 1982) 33], Huber and Strassen (1972) 62] and the references therein for more general case. It seems that the notion of upper expectations was first discussed by Huber (1981) 61] in robust statistics. Recently, it was rediscovered in mathematical finance, especially in risk measure, see Delbaen $(1992,2002)$ [34, 35], Föllmer and Schied $(2002,2004)$ [50] and etc.. 


\section{Appendix A Preliminaries in Functional Analysis}

\section{$\S 1$ Completion of Normed Linear Spaces}

In this section, we suppose $\mathcal{H}$ is a linear space under the norm $\|\cdot\|$.

Definition 1.1 $\left\{x_{n}\right\} \in \mathcal{H}$ is a Cauchy sequence, if $\left\{x_{n}\right\}$ satisfies Cauchy's convergence condition:

$$
\lim _{n, m \rightarrow \infty}\left\|x_{n}-x_{m}\right\|=0
$$

Definition 1.2 A normed linear space $\mathcal{H}$ is called a Banach space if it is complete, i.e., if every Cauchy sequence $\left\{x_{n}\right\}$ of $\mathcal{H}$ converges strongly to a point $x_{\infty}$ of $\mathcal{H}$ :

$$
\lim _{n \rightarrow \infty}\left\|x_{n}-x_{\infty}\right\|=0 .
$$

Such a limit point $x_{\infty}$, if exists, is uniquely determined because of the triangle inequality $\left\|x-x^{\prime}\right\| \leq\left\|x-x_{n}\right\|+\left\|x_{n}-x^{\prime}\right\|$.

The completeness of a Banach space plays an important role in functional analysis. We introduce the following theorem of completion.

Theorem 1.3 Let $\mathcal{H}$ be a normed linear space which is not complete. Then $\mathcal{H}$ is isomorphic and isometric to a dense linear subspace of a Banach-space $\tilde{\mathcal{H}}$, i.e., there exists a one-to-one correspondence $x \leftrightarrow \tilde{x}$ of $\mathcal{H}$ onto a dense linear subspace of $\tilde{\mathcal{H}}$ such that

$$
\widetilde{x+y}=\tilde{x}+\tilde{y}, \widetilde{\alpha x}=\alpha \tilde{x},\|\tilde{x}\|=\|x\| .
$$

The space $\tilde{\mathcal{H}}$ is uniquely determined up to isometric isomorphism.

For a proof see Yosida [125] (1980, p.56). 


\section{$\S 2$ The Hahn-Banach Extension Theorem}

Definition 2.1 Let $T_{1}$ and $T_{2}$ be two linear operators with domains $D\left(T_{1}\right)$ and $D\left(T_{2}\right)$ both contained in a linear space $\mathcal{H}$, and the ranges $R\left(T_{1}\right)$ and $R\left(T_{2}\right)$ both contained in a linear space $\mathcal{M}$. Then $T_{1}=T_{2}$ if and only if $D\left(T_{1}\right)=D\left(T_{2}\right)$ and $T_{1} x=T_{2} x$ for all $x \in D\left(T_{1}\right)$. If $D\left(T_{1}\right) \subseteq D\left(T_{2}\right)$ and $T_{1} x=T_{2} x$ for all $x \in D\left(T_{1}\right)$, then $T_{2}$ is called an extension of $T_{1}$, or $T_{1}$ is called a restriction of $T_{2}$.

Theorem 2.2 (Hahn-Banach extension theorem in real linear spaces) Let $\mathcal{H}$ be a real linear space and let $p(x)$ be a real-valued function defined on $\mathcal{H}$ satisfying the following conditions:

$$
\begin{aligned}
& p(x+y) \leq p(x)+p(y) \quad \text { (subadditivity); } \\
& p(\alpha x)=\alpha p(x) \text { for } \alpha \geq 0 \text { (positive homogeneity). }
\end{aligned}
$$

Let $\mathcal{L}$ be a real linear subspace of $\mathcal{H}$ and $f_{0}$ be a real-valued linear functional defined on $\mathcal{L}$ :

$$
f_{0}(\alpha x+\beta y)=\alpha f_{0}(x)+\beta f_{0}(y) \text { for } x, y \in \mathcal{L} \text { and } \alpha, \beta \in \mathbb{R} .
$$

Let $f_{0}$ satisfy $f_{0}(x) \leq p(x)$ on $\mathcal{L}$. Then there exists a real-valued linear functional $F$ defined on $\mathcal{H}$ such that

(i) $F$ is an extension of $f_{0}$, i.e., $F(x)=f_{0}(x)$ for all $x \in \mathcal{L}$.

(ii) $F(x) \leq p(x) \quad$ for $x \in \mathcal{H}$.

For a proof see Yosida [125] (1980, p.102).

Theorem 2.3 (Hahn-Banach extension theorem in normed linear spaces) Let $\mathcal{H}$ be a normed linear space under the norm $\|\cdot\|, \mathcal{L}$ be a linear subspace of $\mathcal{H}$ and let $f_{1}$ be a continuous linear functional defined on $\mathcal{L}$. Then there exists a continuous linear functional $f$, defined on $\mathcal{H}$, such that

(i) $f$ is an extension of $f_{1}$.

(ii) $\left\|f_{1}\right\|=\|f\|$.

For a proof see for example Yosida [125] (1980, p.106).

\section{$\S 3$ Dini's Theorem and Tietze's Extension The- orem}

Theorem 3.1 (Dini's theorem) Let $\mathcal{H}$ be a compact topological space. If a monotone sequence of bounded continuous functions converges pointwise to a continuous function, then it also converges uniformly.

Theorem 3.2 (Tietze's extension theorem) Let $\mathcal{L}$ be a closed subset of a normal space $\mathcal{H}$ and let $f: \mathcal{L} \rightarrow \mathbb{R}$ be a continuous function. Then there exists a continuous extension of $f$ to all of $\mathcal{H}$ with values in $\mathbb{R}$. 


\section{Appendix B Preliminaries in Probability Theory}

\section{$\S 1$ Kolmogorov's Extension Theorem}

Let $X$ be a random variable with values in $\mathbb{R}^{n}$ defined on a probability space $(\Omega, \mathcal{F}, P)$. Denote by $\mathcal{B}$ the Borel $\sigma$-algebra on $\mathbb{R}^{n}$. We define $X$ 's law of distribution $P_{X}$ and its expectation $E_{P}$ with respect to $P$ as follows respectively:

$$
P_{X}(B):=P(\omega: X(\omega) \in B) ; E_{P}[X]:=\int_{-\infty}^{+\infty} x P(d x),
$$

where $B \in \mathcal{B}$.

In fact, we have $P_{X}(B)=E_{P}\left[\mathbf{I}_{B}(X)\right]$.

Now let $\left\{X_{t}\right\}_{t \in T}$ be a stochastic process with values in $\mathbb{R}^{n}$ defined on a probability space $(\Omega, \mathcal{F}, P)$, where the parameter space $T$ is usually the halfline $[0,+\infty)$.

Definition 1.1 The finite dimensional distributions of the process $\left\{X_{t}\right\}_{t \in T}$ are the measures $\mu_{t_{1}, \cdots, t_{k}}$ defined on $\mathbb{R}^{n k}, k=1,2, \cdots$, by

$\mu_{t_{1}, \cdots, t_{k}}\left(B_{1} \times \cdots \times B_{k}\right):=P\left[X_{t_{1}} \in B_{1}, \cdots, X_{t_{k}} \in B_{k}\right], t_{i} \in T, i=1,2, \cdots, k$,

where $B_{i} \in \mathcal{B}, i=1,2, \cdots, k$.

The family of all finite-dimensional distributions determine many (but not all) important properties of the process $\left\{X_{t}\right\}_{t \in T}$.

Conversely, given a family $\left\{\nu_{t_{1}, \cdots, t_{k}}: t_{i} \in T, i=1,2, \cdots, k, k \in \mathbb{N}\right\}$ of probability measures on $\mathbb{R}^{n k}$, it is important to be able to construct a stochastic process $\left(Y_{t}\right)_{t \in T}$ with $\nu_{t_{1}, \cdots, t_{k}}$ as its finite-dimensional distributions. The following famous theorem states that this can be done provided that $\left\{\nu_{t_{1}, \cdots, t_{k}}\right\}$ satisfy two natural consistency conditions. 
Theorem 1.2 (Kolmogorov's extension theorem) For all $t_{1}, t_{2}, \cdots, t_{k}$, $k \in \mathbb{N}$, let $\nu_{t_{1}, \cdots, t_{k}}$ be probability measures on $\mathbb{R}^{n k}$ such that

$$
\nu_{t_{\pi(1)}, \cdots, t_{\pi(k)}}\left(B_{1} \times \cdots \times B_{k}\right)=\nu_{t_{1}, \cdots, t_{k}}\left(B_{\pi^{-1}(1)} \times \cdots \times B_{\pi^{-1}(k)}\right)
$$

for all permutations $\pi$ on $\{1,2, \cdots, k\}$ and

$$
\nu_{t_{1}, \cdots, t_{k}}\left(B_{1} \times \cdots \times B_{k}\right)=\nu_{t_{1}, \cdots, t_{k}, t_{k+1}, \cdots, t_{k+m}}\left(B_{1} \times \cdots \times B_{k} \times \mathbb{R}^{n} \times \cdots \times \mathbb{R}^{n}\right)
$$

for all $m \in \mathbb{N}$, where the set on the right hand side has a total of $k+m$ factors. Then there exists a probability space $(\Omega, \mathcal{F}, P)$ and a stochastic process $\left(X_{t}\right)$ on $\Omega, X_{t}: \Omega \rightarrow \mathbb{R}^{n}$, such that

$$
\nu_{t_{1}, \cdots, t_{k}}\left(B_{1} \times \cdots \times B_{k}\right)=P\left[X_{t_{1}} \in B_{1}, \cdots, X_{t_{k}} \in B_{k}\right]
$$

for all $t_{i} \in T$ and all Borel sets $B_{i}, i=1,2, \cdots, k, k \in \mathbb{N}$.

For a proof see Kolmogorov [74] (1956, p.29).

\section{$\S 2$ Kolmogorov's Criterion}

Definition 2.1 Suppose that $\left(X_{t}\right)$ and $\left(Y_{t}\right)$ are two stochastic processes defined on $(\Omega, \mathcal{F}, P)$. Then we say that $\left(X_{t}\right)$ is a version of (or a modification of) $\left(Y_{t}\right)$ if

$$
P\left(\left\{\omega: X_{t}(\omega)=Y_{t}(\omega)\right\}\right)=1 \text { for all } t .
$$

Theorem 2.2 (Kolmogorov's continuity criterion) Suppose that the process $X=\left\{X_{t}\right\}_{t \geq 0}$ satisfies the following condition: for all $T>0$ there exist positive constants $\alpha, \beta, D$ such that

$$
E\left[\left|X_{t}-X_{s}\right|^{\alpha}\right] \leq D|t-s|^{1+\beta}, \quad 0 \leq s, t \leq T .
$$

Then there exists a continuous version of $X$.

For a proof see Stroock and Varadhan 117] (1979, p.51).

Let $E$ be a metric space and $\mathcal{B}$ be the Borel $\sigma$-algebra on $E$. We recall a few facts about the weak convergence of probability measures on $(E, \mathcal{B})$. If $P$ is such a measure, we say that a subset $A$ of $E$ is a $P$-continuity set if $P(\partial A)=0$, where $\partial A$ is the boundary of $A$.

Proposition 2.3 For probability measures $P_{n}(n \in \mathbb{N})$ and $P$, the following conditions are equivalent:

(i) For every bounded continuous function $f$ on $E$,

$$
\lim _{n \rightarrow \infty} \int f d P_{n}=\int f d P
$$


(ii) For every bounded uniformly continuous function $f$ on $E$,

$$
\lim _{n \rightarrow \infty} \int f d P_{n}=\int f d P
$$

(iii) For every closed subset $F$ of $E$, $\limsup _{n \rightarrow \infty} P_{n}(F) \leq P(F)$;

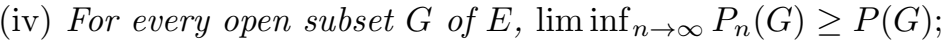

(v) For every $P$-continuity set $A, \lim _{n \rightarrow \infty} P_{n}(A)=P(A)$.

Definition 2.4 If $P_{n}$ and $P$ satisfy the equivalent conditions of the preceding proposition, we say that $\left(P_{n}\right)$ converges weakly to $P$.

Now let $\pi$ be a family of probability measures on $(E, \mathcal{B})$.

Definition 2.5 A family $\pi$ is weakly relatively compact if every sequence of $\pi$ contains a weakly convergent subsequence.

Definition 2.6 A family $\pi$ is tight if for every $\varepsilon \in(0,1)$, there exists a compact set $K_{\varepsilon}$ such that

$$
P\left(K_{\varepsilon}\right) \geq 1-\varepsilon \quad \text { for every } P \in \pi \text {. }
$$

With this definition, we have the following theorem.

Theorem 2.7 (Prokhorov's criterion) If a family $\pi$ is tight, then it is weakly relatively compact. If $E$ is a Polish space (i.e., a separable completely metrizable topological space), then a weakly relatively compact family is tight.

Definition 2.8 If $\left(X_{n}\right)_{n \in \mathbb{N}}$ and $X$ are random variables taking their values in a metric space $E$, we say that $\left(X_{n}\right)$ converges in distribution or converges in law to $X$ if their laws $P_{X_{n}}$ converge weakly to the law $P_{X}$ of $X$.

We stress the fact that the $\left(X_{n}\right)$ and $X$ need not be defined on the same probability space.

Theorem 2.9 (Kolmogorov's criterion for weak compactness) Let $\left\{X^{n}\right\}$ be a sequence of $\mathbb{R}^{d}$-valued continuous processes defined on probability spaces $\left(\Omega^{n}, \mathcal{F}^{n}, P^{n}\right)$ such that

(i) the family $\left\{P_{X_{0}^{n}}^{n}\right\}$ of initial laws is tight in $\mathbb{R}^{d}$.

(ii) there exist three strictly positive constants $\alpha, \beta, \gamma$ such that for each $s, t \in \mathbb{R}_{+}$ and each $n$,

$$
E_{P^{n}}\left[\left|X_{s}^{n}-X_{t}^{n}\right|^{\alpha}\right] \leq \beta|s-t|^{\gamma+1},
$$

then the set $\left(P_{X^{n}}^{n}\right)$ of the laws of the $\left(X_{n}\right)$ is weakly relatively compact.

For the proof see Revuz and Yor [11] (1999, p.517) 


\section{$\S 3 \quad$ Daniell-Stone Theorem}

Let $(\Omega, \mathcal{F}, \mu)$ be a measure space, on which we can define integration. One essential properties of integration is its linearity, thus it can be seen as a linear functional on $L^{1}(\Omega, \mathcal{F}, \mu)$. This idea leads to another approach to define integral-Daniell's integral.

Definition 3.1 Let $\Omega$ be an abstract set and $\mathcal{H}$ be a linear space formed by a family of real valued functions. $\mathcal{H}$ is called a vector lattice if

$$
f \in \mathcal{H} \Rightarrow|f| \in \mathcal{H}, f \wedge 1 \in \mathcal{H} .
$$

Definition 3.2 Suppose that $\mathcal{H}$ is a vector lattice on $\Omega$ and $I$ is a positive linear functional on $\mathcal{H}$, i.e.,

$$
\begin{aligned}
& f, g \in \mathcal{H}, \alpha, \beta \in \mathbb{R} \Rightarrow I(\alpha f+\beta g)=\alpha I(f)+\beta I(g) ; \\
& f \in \mathcal{H}, f \geq 0 \Rightarrow I(f) \geq 0 .
\end{aligned}
$$

If I satisfies the following condition:

$$
f_{n} \in \mathcal{H}, f_{n} \downarrow 0 \Rightarrow I\left(f_{n}\right) \rightarrow 0,
$$

or equivalently,

$$
f_{n} \in \mathcal{H}, f_{n} \uparrow f \in \mathcal{H} \Rightarrow I(f)=\lim _{n \rightarrow \infty} I\left(f_{n}\right),
$$

then I is called a Daniell's integral on $\mathcal{H}$.

Theorem 3.3 (Daniell-Stone theorem) Suppose that $\mathcal{H}$ is a vector lattice on $\Omega$ and $I$ is a Daniell integral on $\mathcal{H}$. Then there exists a measure $\mu \in \mathcal{F}$, where $\mathcal{F}:=\sigma(f: f \in \mathcal{H})$, such that $\mathcal{H} \subset L^{1}(\Omega, \mathcal{F}, \mu)$ and $I(f)=\mu(f), \forall f \in \mathcal{H}$. Furthermore, if $1 \in \mathcal{H}_{+}^{*}$, where $\mathcal{H}_{+}^{*}:=\left\{f: \exists f_{n} \geq 0, f_{n} \in \mathcal{H}\right.$ such that $\left.f_{n} \uparrow f\right\}$, then this measure $\mu$ is unique and is $\sigma$-finite.

For the proof see Dellacherie and Meyer 33] (1978, p.59), Dudley [41] (1995, p.142), or Yan [123] (1998, p.74). 


\section{Appendix $\mathrm{C}$ Solutions of Parabolic Partial Differential Equation}

\section{$\S 1$ The Definition of Viscosity Solutions}

The notion of viscosity solutions was firstly introduced by Crandall and Lions (1981) [28] and (1983) [29] (see also Evans's contribution (1978) 45] and (1980) 46]) for the first-order Hamilton-Jacobi equation, with uniqueness proof given in [29]. The the proof of second-order case for Hamilton-Jacobi-Bellman equations was firstly developed by Lions (1982) 80] and (1983) 81] using stochastic control verification arguments. A breakthrough was achieved in the second-order PDE theory by Jensen (1988) 67]. For all other important contributions in the developments of this theory we refer to the well-known user's guide by Crandall, Ishii and Lions (1992) 30]. For reader's convenience, we systematically interpret some parts of [30] required in this book into it's parabolic version. However, up to my knowledge, the presentation and the related proof for the domination theorems seems to be a new generalization of the maximum principle presented in [30]. Books on this theory are, among others, Barles (1994) [8], Fleming, and Soner (1992) [49], Yong and Zhou (1999) [124].

Let $T>0$ be fixed and let $\mathcal{O} \subset[0, T] \times \mathbb{R}^{N}$. We set

$$
\begin{aligned}
& U S C(\mathcal{O})=\{\text { upper semicontinuous functions } u: \mathcal{O} \rightarrow \mathbb{R}\} \\
& L S C(\mathcal{O})=\{\text { lower semicontinuous functions } u: \mathcal{O} \rightarrow \mathbb{R}\}
\end{aligned}
$$

Consider the following parabolic PDE:

$$
\left\{\begin{array}{l}
\text { (E) } \partial_{t} u-G\left(t, x, u, D u, D^{2} u\right)=0 \text { on }(0, T) \times \mathbb{R}^{N} \\
(\mathrm{IC}) u(0, x)=\varphi(x) \text { for } x \in \mathbb{R}^{N}
\end{array}\right.
$$

where $G:[0, T] \times \mathbb{R}^{N} \times \mathbb{R} \times \mathbb{R}^{N} \times \mathbb{S}(N) \rightarrow \mathbb{R}, \varphi \in C\left(\mathbb{R}^{N}\right)$. We always suppose 
that $G$ is continuous and satisfies the following degenerate elliptic condition:

$$
G(t, x, r, p, X) \geq G(t, x, r, p, Y) \text { whenever } X \geq Y .
$$

Next we recall the definition of viscosity solutions from Crandall, Ishii and Lions 30]. Let $u:(0, T) \times \mathbb{R}^{N} \rightarrow \mathbb{R}$ and $(t, x) \in(0, T) \times \mathbb{R}^{N}$. We denote by $\mathcal{P}^{2,+} u(t, x)$ (the "parabolic superjet" of $u$ at $(t, x))$ the set of triples $(a, p, X) \in \mathbb{R} \times \mathbb{R}^{N} \times$ $\mathbb{S}(N)$ such that

$$
\begin{aligned}
u(s, y) \leq & u(t, x)+a(s-t)+\langle p, y-x\rangle \\
& +\frac{1}{2}\langle X(y-x), y-x\rangle+o\left(|s-t|+|y-x|^{2}\right) .
\end{aligned}
$$

We define

$$
\begin{aligned}
\overline{\mathcal{P}}^{2,+} u(t, x):=\{( & (a, p, X) \in \mathbb{R} \times \mathbb{R}^{N} \times \mathbb{S}(N): \exists\left(t_{n}, x_{n}, a_{n}, p_{n}, X_{n}\right) \\
& \text { such that }\left(a_{n}, p_{n}, X_{n}\right) \in \mathcal{P}^{2,+} u\left(t_{n}, x_{n}\right) \text { and } \\
& \left.\left(t_{n}, x_{n}, u\left(t_{n}, x_{n}\right), a_{n}, p_{n}, X_{n}\right) \rightarrow(t, x, u(t, x), a, p, X)\right\} .
\end{aligned}
$$

Similarly, we define $\mathcal{P}^{2,-} u(t, x)$ (the "parabolic subjet" of $u$ at $(t, x)$ ) by $\mathcal{P}^{2,-} u(t, x):=-\mathcal{P}^{2,+}(-u)(t, x)$ and $\overline{\mathcal{P}}^{2,-} u(t, x)$ by $\overline{\mathcal{P}}^{2,-} u(t, x):=-\overline{\mathcal{P}}^{2,+}(-u)(t, x)$.

Definition 1.1 (i) A viscosity subsolution of $(E)$ on $(0, T) \times \mathbb{R}^{N}$ is a function $u \in U S C\left((0, T) \times \mathbb{R}^{N}\right)$ such that for each $(t, x) \in(0, T) \times \mathbb{R}^{N}$,

$$
a-G(t, x, u(t, x), p, X) \leq 0 \text { for }(a, p, X) \in \mathcal{P}^{2,+} u(t, x) ;
$$

likewise, a viscosity supersolution of $(E)$ on $(0, T) \times \mathbb{R}^{N}$ is a function $v \in$ $L S C\left((0, T) \times \mathbb{R}^{N}\right)$ such that for each $(t, x) \in(0, T) \times \mathbb{R}^{N}$,

$$
a-G(t, x, v(t, x), p, X) \geq 0 \quad \text { for }(a, p, X) \in \mathcal{P}^{2,-} v(t, x)
$$

and a viscosity solution of $(E)$ on $(0, T) \times \mathbb{R}^{N}$ is a function that is simultaneously a viscosity subsolution and a viscosity supersolution of $(E)$ on $(0, T) \times \mathbb{R}^{N}$. (ii) A function $u \in U S C\left([0, T) \times \mathbb{R}^{N}\right)$ is called a viscosity subsolution of (1.1) on $[0, T) \times \mathbb{R}^{N}$ if $u$ is a viscosity subsolution of $(E)$ on $(0, T) \times \mathbb{R}^{N}$ and $u(0, x) \leq \varphi(x)$ for $x \in \mathbb{R}^{N}$; the appropriate notions of a viscosity supersolution and a viscosity solution of (1.1) on $[0, T) \times \mathbb{R}^{N}$ are then obvious.

We now give the following equivalent definition (see Crandall, Ishii and Lions [30]).

Definition 1.2 A viscosity subsolution of $(E)$, or $G$-subsolution, on $(0, T) \times \mathbb{R}^{N}$ is a function $u \in U S C\left((0, T) \times \mathbb{R}^{N}\right)$ such that for all $(t, x) \in(0, T) \times \mathbb{R}^{N}$, $\phi \in C^{2}\left((0, T) \times \mathbb{R}^{N}\right)$ such that $u(t, x)=\phi(t, x)$ and $u<\phi$ on $(0, T) \times \mathbb{R}^{N} \backslash(t, x)$, we have

$$
\partial_{t} \phi(t, x)-G\left(t, x, \phi(t, x), D \phi(t, x), D^{2} \phi(t, x)\right) \leq 0 ;
$$


likewise, a viscosity supersolution of $(E)$, or $G$-supersolution, on $(0, T) \times \mathbb{R}^{N}$ is a function $v \in L S C\left((0, T) \times \mathbb{R}^{N}\right)$ such that for all $(t, x) \in(0, T) \times \mathbb{R}^{N}$, $\phi \in C^{2}\left((0, T) \times \mathbb{R}^{N}\right)$ such that $u(t, x)=\phi(t, x)$ and $u>\phi$ on $(0, T) \times \mathbb{R}^{N} \backslash(t, x)$, we have

$$
\partial_{t} \phi(t, x)-G\left(t, x, \phi(t, x), D \phi(t, x), D^{2} \phi(t, x)\right) \geq 0 ;
$$

and a viscosity solution of $(E)$ on $(0, T) \times \mathbb{R}^{N}$ is a function that is simultaneously a viscosity subsolution and a viscosity supersolution of $(E)$ on $(0, T) \times \mathbb{R}^{N}$. The definition of a viscosity solution of (1.1) on $[0, T) \times \mathbb{R}^{N}$ is the same as the above definition.

\section{$\S 2$ Comparison Theorem}

We will use the following well-known result in viscosity solution theory (see Theorem 8.3 of Crandall, Ishii and Lions [30]).

Theorem 2.1 Let $u_{i} \in U S C\left((0, T) \times \mathbb{R}^{N_{i}}\right)$ for $i=1, \cdots, k$. Let $\varphi$ be a function defined on $(0, T) \times \mathbb{R}^{N_{1}+\cdots+N_{k}}$ such that $\left(t, x_{1}, \ldots, x_{k}\right) \rightarrow \varphi\left(t, x_{1}, \ldots, x_{k}\right)$ is once continuously differentiable in $t$ and twice continuously differentiable in $\left(x_{1}, \cdots, x_{k}\right) \in \mathbb{R}^{N_{1}+\cdots+N_{k}}$. Suppose that $\hat{t} \in(0, T), \hat{x}_{i} \in \mathbb{R}^{N_{i}}$ for $i=1, \cdots, k$ and

$$
\begin{aligned}
w\left(t, x_{1}, \cdots, x_{k}\right) & :=u_{1}\left(t, x_{1}\right)+\cdots+u_{k}\left(t, x_{k}\right)-\varphi\left(t, x_{1}, \cdots, x_{k}\right) \\
& \leq w\left(\hat{t}, \hat{x}_{1}, \cdots, \hat{x}_{k}\right)
\end{aligned}
$$

for $t \in(0, T)$ and $x_{i} \in \mathbb{R}^{N_{i}}$. Assume, moreover, that there exists $r>0$ such that for every $M>0$ there exists constant $C$ such that for $i=1, \cdots, k$,

$$
\begin{aligned}
& b_{i} \leq C \text { whenever }\left(b_{i}, q_{i}, X_{i}\right) \in \mathcal{P}^{2,+} u_{i}\left(t, x_{i}\right) \\
& \left|x_{i}-\hat{x}_{i}\right|+|t-\hat{t}| \leq r \text { and }\left|u_{i}\left(t, x_{i}\right)\right|+\left|q_{i}\right|+\left\|X_{i}\right\| \leq M .
\end{aligned}
$$

Then for each $\varepsilon>0$, there exist $X_{i} \in \mathbb{S}\left(N_{i}\right)$ such that

(i) $\left(b_{i}, D_{x_{i}} \varphi\left(\hat{t}, \hat{x}_{1}, \cdots, \hat{x}_{k}\right), X_{i}\right) \in \overline{\mathcal{P}}^{2,+} u_{i}\left(\hat{t}, \hat{x}_{i}\right), \quad i=1, \cdots, k$,

(ii)

$$
-\left(\frac{1}{\varepsilon}+\|A\|\right) I \leq\left[\begin{array}{ccc}
X_{1} & \cdots & 0 \\
\vdots & \ddots & \vdots \\
0 & \cdots & X_{k}
\end{array}\right] \leq A+\varepsilon A^{2},
$$

(iii) $b_{1}+\cdots+b_{k}=\partial_{t} \varphi\left(\hat{t}, \hat{x}_{1}, \cdots, \hat{x}_{k}\right)$, where $A=D_{x}^{2} \varphi(\hat{t}, \hat{x}) \in \mathbb{S}\left(N_{1}+\cdots+N_{k}\right)$.

Observe that the above condition (2.3) will be guaranteed by having each $u_{i}$ be a subsolution of a parabolic equation given in the following two theorems. 
In this section we will give comparison theorem for $G$-solutions with different functions $G$.

(G) We assume that

$$
G:[0, T] \times \mathbb{R}^{N} \times \mathbb{R} \times \mathbb{R}^{N} \times \mathbb{S}(N) \rightarrow \mathbb{R}, \quad i=1, \cdots, k,
$$

are continuous in the following sense: for each $t \in[0, T), v \in \mathbb{R}, x, y, p \in \mathbb{R}^{N}$ and $X \in \mathbb{S}(N)$,

$$
\begin{aligned}
& \left|G_{i}(t, x, v, p, X)-G_{i}(t, y, v, p, X)\right| \\
& \leq \bar{\omega}\left(1+(T-t)^{-1}+|x|+|y|+|v|\right) \omega(|x-y|+|p| \cdot|x-y|),
\end{aligned}
$$

where $\omega, \bar{\omega}: \mathbb{R}^{+} \rightarrow \mathbb{R}^{+}$are given continuous functions with $\omega(0)=0$.

Theorem 2.2 (Domination Theorem) We are given constants $\beta_{i}>0, i=$ $1, \cdots, k$. Let $u_{i} \in U S C\left([0, T] \times \mathbb{R}^{N}\right)$ be subsolutions of

$$
\partial_{t} u-G_{i}\left(t, x, u, D u, D^{2} u\right)=0, \quad i=1, \cdots, k,
$$

on $(0, T) \times \mathbb{R}^{N}$ such that $\left(\sum_{i=1}^{k} \beta_{i} u_{i}(t, x)\right)^{+} \rightarrow 0$, uniformly as $|x| \rightarrow \infty$. We assume that the functions $\left\{G_{i}\right\}_{i}=1^{k}$ satisfies assumption $(G)$ and the following domination condition holds for:

$$
\sum_{i=1}^{k} \beta_{i} G_{i}\left(t, x, v_{i}, p_{i}, X_{i}\right) \leq 0,
$$

for each $(t, x) \in(0, T) \times \mathbb{R}^{N}$ and $\left(v_{i}, p_{i}, X_{i}\right) \in \mathbb{R} \times \mathbb{R}^{N} \times \mathbb{S}(N)$ such that $\sum_{i=1}^{k} \beta_{i} v_{i} \geq 0, \sum_{i=1}^{k} \beta_{i} p_{i}=0, \sum_{i=1}^{k} \beta_{i} X_{i} \leq 0$.

Then a domination also holds for the solutions: if the sum of initial values $\sum_{i=1}^{k} \beta_{i} u_{i}(0, \cdot)$ is a non-positive function on $\mathbb{R}^{N}$, then $\sum_{i=1}^{k} \beta_{i} u_{i}(t, \cdot) \leq 0$, for all $t>0$.

Proof. We first observe that for $\bar{\delta}>0$ and for each $1 \leq i \leq k$, the functions defined by $\tilde{u}_{i}:=u_{i}-\bar{\delta} /(T-t)$ is a subsolution of

$$
\partial_{t} \tilde{u}_{i}-\tilde{G}_{i}\left(t, x, \tilde{u}_{i}, D \tilde{u}_{i}, D^{2} \tilde{u}_{i}\right) \leq-\frac{\bar{\delta}}{(T-t)^{2}},
$$

where $\tilde{G}_{i}(t, x, v, p, X):=G_{i}(t, x, v+\bar{\delta} /(T-t), p, X)$. It is easy to check that the functions $\tilde{G}_{i}$ satisfy the same conditions as $G_{i}$. Since $\sum_{i=1}^{k} \beta_{i} u_{i} \leq 0$ follows from $\sum_{i=2}^{k} \beta_{i} \tilde{u}_{i} \leq 0$ in the limit $\bar{\delta} \downarrow 0$, it suffices to prove the theorem under the additional assumptions:

$$
\begin{aligned}
& \partial_{t} u_{i}-G_{i}\left(t, x, u_{i}, D u_{i}, D^{2} u_{i}\right) \leq-c, \text { where } c=\bar{\delta} / T^{2}, \\
& \text { and } \lim _{t \rightarrow T} u_{i}(t, x)=-\infty \text { uniformly on }[0, T) \times \mathbb{R}^{N} .
\end{aligned}
$$


To prove the theorem, we assume to the contrary that

$$
\sup _{(t, x) \in[0, T) \times \mathbb{R}^{N}} \sum_{i=1}^{k} \beta_{i} u_{i}(t, x)=m_{0}>0
$$

We will apply Theorem 2.1 for $x=\left(x_{1}, \cdots, x_{k}\right) \in \mathbb{R}^{k \times N}$ and

$$
w(t, x):=\sum_{i=1}^{k} \beta_{i} u_{i}\left(t, x_{i}\right), \quad \varphi(x)=\varphi_{\alpha}(x):=\frac{\alpha}{2} \sum_{i=1}^{k-1}\left|x_{i+1}-x_{i}\right|^{2} .
$$

For each large $\alpha>0$, the maximum of $w-\varphi_{\alpha}$ achieves at some $\left(t^{\alpha}, x^{\alpha}\right)$ inside a compact subset of $[0, T) \times \mathbb{R}^{k \times N}$. Indeed, since

$$
M_{\alpha}=\sum_{i=1}^{k} \beta_{i} u_{i}\left(t^{\alpha}, x_{i}^{\alpha}\right)-\varphi_{\alpha}\left(x^{\alpha}\right) \geq m_{0},
$$

we conclude $t^{\alpha}$ must be inside an interval $\left[0, T_{0}\right], T_{0}<T$ and $x^{\alpha}$ must be inside a compact set $\left\{x \in \mathbb{R}^{k \times N}: \sup _{t \in\left[0, T_{0}\right]} w(t, x) \geq \frac{m_{0}}{2}\right\}$. We can check that (see [30] Lemma 3.1)

$$
\left\{\begin{aligned}
\text { (i) } \lim _{\alpha \rightarrow \infty} \varphi_{\alpha}\left(x^{\alpha}\right)=0 \\
\text { (ii) } \begin{array}{rl}
\lim _{\alpha \rightarrow \infty} M_{\alpha} & \left.=\lim _{\alpha \rightarrow \infty} \beta_{1} u_{1}\left(t^{\alpha}, x_{1}^{\alpha}\right)+\cdots+\beta_{k} u_{k}\left(t^{\alpha}, x_{k}^{\alpha}\right)\right) \\
& =\sup _{(t, x) \in[0, T) \times \mathbb{R}^{N}}\left[\beta_{1} u_{1}(t, x)+\cdots+\beta_{k} u_{k}(t, x)\right] \\
& =\left[\beta_{1} u_{1}(\hat{t}, \hat{x})+\cdots+\beta_{k} u_{k}(\hat{t}, \hat{x})\right]=m_{0},
\end{array}
\end{aligned}\right.
$$

where $(\hat{t}, \hat{x})$ is a limit point of $\left(t^{\alpha}, x^{\alpha}\right)$. Since $u_{i} \in \mathrm{USC}$, for sufficiently large $\alpha$, we have

$$
\beta_{1} u_{1}\left(t^{\alpha}, x_{1}^{\alpha}\right)+\cdots+\beta_{k} u_{k}\left(t^{\alpha}, x_{k}^{\alpha}\right) \geq \frac{m_{0}}{2} .
$$

If $\hat{t}=0$, we have $\lim \sup _{\alpha \rightarrow \infty} \sum_{i=1}^{k} \beta_{i} u_{i}\left(t^{\alpha}, x_{i}^{\alpha}\right)=\sum_{i=1}^{k} \beta_{i} u_{i}(0, \hat{x}) \leq 0$. We know that $\hat{t}>0$ and thus $t^{\alpha}$ must be strictly positive for large $\alpha$. It follows from Theorem 2.1 that, for each $\varepsilon>0$ there exist $b_{i}^{\alpha} \in \mathbb{R}, X_{i} \in \mathbb{S}(N)$ such that

$$
\left(b_{i}^{\alpha}, \beta_{i}^{-1} D_{x_{i}} \varphi\left(x^{\alpha}\right), X_{i}\right) \in \overline{\mathcal{P}}^{2,+} u_{i}\left(t^{\alpha}, x_{i}^{\alpha}\right), \quad \sum_{i=1}^{k} \beta_{i} b_{i}^{\alpha}=0 \text { for } i=1, \cdots, k,
$$

and such that

$$
-\left(\frac{1}{\varepsilon}+\|A\|\right) I \leq\left(\begin{array}{cccc}
\beta_{1} X_{1} & \ldots & 0 & 0 \\
\vdots & \ddots & \vdots & \vdots \\
0 & \ldots & \beta_{k-1} X_{k-1} & 0 \\
0 & \ldots & 0 & \beta_{k} X_{k}
\end{array}\right) \leq A+\varepsilon A^{2},
$$


where $A=D^{2} \varphi_{\alpha}\left(x^{\alpha}\right) \in \mathbb{S}(k N)$ is explicitly given by

$$
A=\alpha J_{k N}, \text { where } J_{k N}=\left(\begin{array}{ccccc}
I_{N} & -I_{N} & \cdots & \cdots & 0 \\
-I_{N} & 2 I_{N} & \ddots & & \vdots \\
\vdots & \ddots & \ddots & \ddots & \vdots \\
\vdots & & \ddots & 2 I_{N} & -I_{N} \\
0 & \cdots & \cdots & -I_{N} & I_{N}
\end{array}\right) .
$$

The second inequality of (2.9) implies $\sum_{i=1}^{k} \beta_{i} X_{i} \leq 0$. Set

$$
\begin{aligned}
p_{1}^{\alpha} & =\beta_{1}^{-1} D_{x_{1}} \varphi_{\alpha}\left(x^{\alpha}\right)=\beta_{1}^{-1} \alpha\left(x_{1}^{\alpha}-x_{2}^{\alpha}\right), \\
p_{2}^{\alpha} & =\beta_{2}^{-1} D_{x_{2}} \varphi_{\alpha}\left(x^{\alpha}\right)=\beta_{2}^{-1} \alpha\left(2 x_{2}^{\alpha}-x_{1}^{\alpha}-x_{3}^{\alpha}\right), \\
& \vdots \\
p_{k-1}^{\alpha} & =\beta_{k-1}^{-1} D_{x_{k-1}} \varphi_{\alpha}\left(x^{\alpha}\right)=\beta_{k-1}^{-1} \alpha\left(2 x_{k-1}^{\alpha}-x_{k-2}^{\alpha}-x_{k}^{\alpha}\right), \\
p_{k}^{\alpha} & =\beta_{k}^{-1} D_{x_{k}} \varphi_{\alpha}\left(x^{\alpha}\right)=\beta_{k}^{-1} \alpha\left(x_{k}^{\alpha}-x_{k-1}^{\alpha}\right) .
\end{aligned}
$$

Thus $\sum_{i=1}^{k} \beta_{i} p_{i}^{\alpha}=0$. From this together with (2.8) and (2.6), it follows that

$$
b_{i}^{\alpha}-G_{i}\left(t^{\alpha}, x_{i}^{\alpha}, u_{i}\left(t^{\alpha}, x_{i}^{\alpha}\right), p_{i}^{\alpha}, X_{i}\right) \leq-c, \quad i=1, \cdots, k .
$$

By (2.7) (i), we also have $\lim _{\alpha \rightarrow \infty}\left|p_{i}^{\alpha}\right| \cdot\left|x_{i}^{\alpha}-x_{1}^{\alpha}\right| \rightarrow 0$. This, together with the domination condition (2.5) of $G_{i}$, implies

$$
\begin{aligned}
-c \sum_{i=1}^{k} \beta_{i}= & -\sum_{i=1}^{k} \beta_{i} b_{i}^{\alpha}-c \sum_{i=1}^{k} \beta_{i} \geq-\sum_{i=1}^{k} \beta_{i} G_{i}\left(t^{\alpha}, x_{i}^{\alpha}, u_{i}\left(t^{\alpha}, x_{i}^{\alpha}\right), p_{i}^{\alpha}, X_{i}\right) \\
\geq & -\sum_{i=1}^{k} \beta_{i} G_{i}\left(t^{\alpha}, x_{1}^{\alpha}, u_{i}\left(t^{\alpha}, x_{i}^{\alpha}\right), p_{i}^{\alpha}, X_{i}\right) \\
& -\sum_{i=1}^{k} \beta_{i}\left|G_{i}\left(t^{\alpha}, x_{i}^{\alpha}, u_{i}\left(t^{\alpha}, x_{i}^{\alpha}\right), p_{i}^{\alpha}, X_{i}\right)-G_{i}\left(t^{\alpha}, x_{1}^{\alpha}, u_{i}\left(t^{\alpha}, x_{i}^{\alpha}\right), p_{i}^{\alpha}, X_{i}\right)\right| \\
\geq & -\sum_{i=1}^{k} \beta_{i} \bar{\omega}\left(1+\left(T-T_{0}\right)^{-1}+\left|x_{1}^{\alpha}\right|+\left|x_{i}^{\alpha}\right|+\left|u_{i}\left(t^{\alpha}, x_{i}^{\alpha}\right)\right|\right) \cdot \omega\left(\left|x_{i}^{\alpha}-x_{1}^{\alpha}\right|\right. \\
& \left.\quad+\left|p_{i}^{\alpha}\right| \cdot\left|x_{i}^{\alpha}-x_{1}^{\alpha}\right|\right) .
\end{aligned}
$$

The right side tends to zero as $\alpha \rightarrow \infty$, which induces a contradiction. The proof is complete.

Theorem 2.3 We assume that the functions $G_{i}=G_{i}(t, x, v, p, X), i=1, \cdots, k$ and $G=G(t, x, v, p, X)$ satisfy assumption $(G)$. We also assume $G$ dominates $\left\{G_{i}\right\}_{i=1}^{k}$ in the following sense: for each $(t, x) \in[0, \infty) \times \mathbb{R}^{N}$ and $\left(v_{i}, p_{i}, X_{i}\right) \in$ 
$\mathbb{R} \times \mathbb{R}^{N} \times \mathbb{S}(N)$

$$
\sum_{i=1}^{k} G_{i}\left(t, x, v_{i}, p_{i}, X_{i}\right) \leq G\left(t, x, \sum_{i=1}^{k} v_{i}, \sum_{i=1}^{k} p_{i}, \sum_{i=1}^{k} X_{i}\right) .
$$

Moreover there exists a constant $\bar{C}$ such that, for each $(t, r, x, p) \in[0, \infty) \times$ $\mathbb{R}^{N} \times \mathbb{R}^{N}$ and, $Y_{1}, Y_{2} \in \mathbb{S}(N)$ such that $Y_{2} \geq Y_{1}$

$$
G\left(t, x, r, p, Y_{2}\right)-G\left(t, x, r_{1}, p, Y_{1}\right) \geq 0
$$

and there exists a constant $\bar{C}$ such that

$$
\left|G\left(t, x, r, p, X_{1}\right)-G\left(t, x, r, p, X_{2}\right)\right| \leq \bar{C}\left(\left|r_{1}-r_{2}\right|+\left|X_{1}-X_{2}\right|\right) .
$$

Let $u_{i} \in U S C\left([0, T] \times \mathbb{R}^{N}\right)$ be a $G_{i}$-subsolution and $u \in L S C\left([0, T] \times \mathbb{R}^{N}\right)$ be a $G$-supersolution such that $u_{i}$ and $u$ satisfy polynomial growth condition. Then $\sum_{i=1}^{k} u_{i}(t, x) \leq u(t, x)$ on $[0, T) \times \mathbb{R}^{N}$ provided that $\left.\sum_{i=1}^{k} u_{i}\right|_{t=0} \leq\left. u\right|_{t=0}$.

Proof. For a fixed and large constant $\lambda>\bar{C}+C$ we set $\xi(x):=\left(1+|x|^{2}\right)^{l / 2}$ and

$$
\tilde{u}_{i}(t, x):=u_{i}(t, x) \xi(x) e^{-\lambda t}, i=1, \cdots, k, \quad \tilde{u}_{k+1}(t, x):=-u(t, x) \xi(x) e^{-\lambda t},
$$

where $l$ is chosen to be large enough such that $\sum\left|\tilde{u}_{i}(t, x)\right| \rightarrow 0$ uniformly as $|x| \rightarrow \infty$. It is easy to check that, for each $i=1, \cdots, k+1, \tilde{u}_{i}$ is a subsolution of

$$
\partial_{t} \tilde{u}_{i}+\lambda \tilde{u}_{i}-\tilde{G}_{i}\left(t, x, \tilde{u}_{i}, D \tilde{u}_{i}, D^{2} \tilde{u}_{i}\right)=0,
$$

where, for each $i=1, \cdots, k$, the function $\tilde{G}_{i}(t, x, v, p, X)$ is given by

$$
e^{-\lambda t} \xi^{-1} G_{i}\left(t, x, e^{\lambda t} v \xi, e^{\lambda t}(p+v D \xi), e^{\lambda t}\left(X \xi+p \otimes D \xi+D \xi \otimes p+v D^{2} \xi\right)\right),
$$

and $\tilde{G}_{k+1}(t, x, v, p, X)$ is given by

$-e^{-\lambda t} \xi^{-1} G\left(t, x,-e^{\lambda t} v \xi,-e^{\lambda t}(p+v D \xi),-e^{\lambda t}\left(X \xi+p \otimes D \xi+D \xi \otimes p+v D^{2} \xi\right)\right)$.

Observe that

$$
\begin{aligned}
D \xi(x) & =l \xi(x)\left(1+|x|^{2}\right)^{-1} x, \\
D^{2} \xi(x) & =\xi(x)\left[l\left(1+|x|^{2}\right)^{-1} I+l(l-2)\left(1+|x|^{2}\right)^{-2} x \otimes x\right] .
\end{aligned}
$$

Thus both $\xi^{-1}(x)|D \xi(x)|$ and $\xi^{-1}(x)\left|D^{2} \xi(x)\right|$ converges to zero uniformly as $|x| \rightarrow \infty$.

From the domination condition (2.10), for each $\left(v_{i}, p_{i}, X_{i}\right) \in \mathbb{R} \times \mathbb{R}^{N} \times \mathbb{S}(N)$, $i=1, \cdots, k+1$, such that $\sum_{i=1}^{k+1} v_{i}=0, \sum_{i=1}^{k+1} p_{i}=0$, and $\sum_{i=1}^{k+1} X_{i}=0$, we have

$$
\sum_{i=1}^{k+1} \tilde{G}_{i}\left(t, x, v_{i}, p_{i}, X_{i}\right) \leq 0
$$


For $v, r \in \mathbb{R}, p \in \mathbb{R}^{N}$ and $X, Y \in \mathbb{S}(N)$ such that $r \geq 0, Y \geq 0$ and $r>0$, since $\tilde{G}$ is still monotone in $X$,

$$
\begin{aligned}
& \tilde{G}_{k+1}(t, x, v, p, X)-\tilde{G}_{k+1}(t, x, v-r, p, X+Y) \\
\leq & \tilde{G}_{k+1}(t, x, v, p, X)-\tilde{G}_{k+1}(t, x, v-r, p, X) \\
\leq & c\left(\hat{C}+C_{1}\right) r
\end{aligned}
$$

where the constant $C_{1}$ does not depend on $(t, x, v, p, X)$. We then apply the above theorem by choosing $\beta_{i}=1, i=1, \cdots, k+1$. Thus $\left.\sum_{i=1}^{k+1} \tilde{u}_{i}\right|_{t=0} \leq 0$. Moreover for each $v_{i} \in \mathbb{R}, p_{i} \in \mathbb{R}^{N}$ and $X_{i} \in \mathbb{S}(N)$ such that $\hat{v}=\sum_{i=1}^{k+1} v_{i} \geq 0$, $\sum_{i=1}^{k+1} p_{i}=0$ and $\hat{X}=\sum_{i=1}^{k+1} X_{i} \leq 0$ we have

$$
\begin{aligned}
& -\lambda \sum_{i=1}^{k+1} v_{i}+\sum_{i=1}^{k+1} \tilde{G}_{i}\left(t, x, v_{i}, p_{i}, X_{i}\right) \\
= & -\lambda \hat{v}+\sum_{i=1}^{k} \tilde{G}_{i}\left(t, x, v_{i}, p_{i}, X_{i}\right)+\tilde{G}_{k+1}\left(t, x, v_{k+1}-\hat{v}, p_{k+1}, X_{k+1}-\hat{X}\right) \\
& +\tilde{G}_{k+1}\left(t, x, v_{k+1}, p_{k+1}, X_{k+1}\right)-\tilde{G}_{k+1}\left(t, x, v_{k+1}-\hat{v}, p_{k+1}, X_{k+1}-\hat{X}\right) \\
\leq & -\lambda \hat{v}+\tilde{G}_{k+1}\left(t, x, v_{k+1}, p_{k+1}, X_{k+1}\right)-\tilde{G}_{k+1}\left(t, x, v_{k+1}-\hat{v}, p_{k+1}, X_{k+1}\right) \\
\leq & -\lambda \hat{v}+c(\bar{C}+C) \hat{v} \leq 0
\end{aligned}
$$

It follows that all conditions in Theorem 2.2 are satisfied. Thus we have $\sum_{i=1}^{k+1} \tilde{u}_{i} \leq 0$, or equivalently, $\sum_{i=1}^{k+1} u_{i}(t, x) \leq u(t, x)$ for $(t, x) \in[0, T) \times \mathbb{R}^{N}$.

The following comparison theorem is a direct consequence of the above domination theorem.

Theorem 2.4 (Comparison Theorem) We are given two functions $G=G(t, x, v, p, X)$ and $G_{1}=G_{1}(t, x, v, p, X)$ satisfying condition $(G)$. We also assume that, for each $(t, x, v, p, X) \in[0, \infty) \times \mathbb{R}^{N} \times \mathbb{R} \times \mathbb{R}^{N}$ and $Y \in \mathbb{S}(N)$ such that $X \geq Y$,

$$
\begin{aligned}
G(t, x, v, p, X) & \geq G_{1}(t, x, v, p, X), \\
G(t, x, v, p, Y) & \geq G(t, x, v, p, X) .
\end{aligned}
$$

We also assume that $G$ is a uniform Lipschitz function in $v$ and $X$, namely, for each $(t, x) \in[0, \infty) \times \mathbb{R}^{N}$ and $(v, p, X),\left(v^{\prime}, p, X^{\prime}\right) \in \mathbb{R} \times \mathbb{R}^{N} \times \mathbb{S}(N)$,

$$
\left|G(t, x, v, p, X)-G\left(t, x, v^{\prime}, p^{\prime}, X^{\prime}\right)\right| \leq \bar{C}\left(\left|v-v^{\prime}\right|+\left|X-X^{\prime}\right|\right) .
$$

Let $u_{1} \in U S C\left([0, T] \times \mathbb{R}^{N}\right)$ be a $G_{1}$-subsolution and $u \in L S C\left([0, T] \times \mathbb{R}^{N}\right)$ be a G-supersolution on $(0, T) \times \mathbb{R}^{N}$ satisfying the polynomial growth condition. Then $u \geq u_{1}$ on $[0, T) \times \mathbb{R}^{N}$ provided that $\left.u\right|_{t=0} \geq\left. u_{1}\right|_{t=0}$. In particular this comparison holds for the case where $G \equiv G_{1}$, which is a Lipschitz function in $(v, X)$ and satisfies the elliptic condition (2.12).

The following special case of the above domination theorem is also very useful. 
Theorem 2.5 (Domination Theorem) We assume that $G_{1}$ and $G$ satisfy the same conditions given in the previous theorem except that the condition (2.11) is replaced by the following one: for each $(t, x) \in[0, \infty) \times \mathbb{R}^{N}$ and $(v, p, X)$, $\left(v^{\prime}, p^{\prime}, X^{\prime}\right) \in \mathbb{R} \times \mathbb{R}^{N} \times \mathbb{S}(N)$,

$$
G_{1}(t, x, v, p, X)-G_{1}\left(t, x, v^{\prime}, p^{\prime}, X^{\prime}\right) \geq G\left(t, x, v-v^{\prime}, p-p^{\prime}, X-X^{\prime}\right)
$$

Let $u \in U S C\left([0, T] \times \mathbb{R}^{N}\right)$ be a $G_{1}$-subsolution and $v \in L S C\left([0, T] \times \mathbb{R}^{N}\right)$ be a $G_{1}$-supersolution on $(0, T) \times \mathbb{R}^{N}$ and $w$ is a $G$-supersolution. They satisfy the polynomial growth condition. If $\left.(u-v)\right|_{t=0}=\left.w\right|_{t=0}$ then $u-v \leq w$ on $[0, T) \times$ $\mathbb{R}^{N}$.

The following theorem will be frequently used in this book. Let $G: \mathbb{R}^{N} \times \mathbb{S}(N) \rightarrow$ $\mathbb{R}$ be a given continuous sublinear function monotonic in $A \in \mathbb{S}(N)$. Obviously, $G$ satisfies conditions (G) of Theorem 2.3 . We consider the following $G$-equation:

$$
\partial_{t} u-G\left(D u, D^{2} u\right)=0, \quad u(0, x)=\varphi(x)
$$

Theorem 2.6 Let $G: \mathbb{R}^{N} \times \mathbb{S}(N) \rightarrow \mathbb{R}$ be a given continuous sublinear function monotonic in $A \in \mathbb{S}(N)$. Then we have

(i) If $u \in \operatorname{USC}\left([0, T] \times \mathbb{R}^{N}\right)$ with polynomial growth is a viscosity subsolution of (2.13) and $v \in \operatorname{LSC}\left([0, T] \times \mathbb{R}^{N}\right)$ with polynomial growth is a viscosity supersolution of (2.13), then $u \leq v$.

(ii) If $u^{\varphi} \in C\left([0, T] \times \mathbb{R}^{N}\right)$ denotes the polynomial growth solution of (2.13) with initial condition $\varphi$, then $u^{\lambda \varphi}=\lambda u^{\varphi}$ for each $\lambda \geq 0$ and $u^{\varphi+\psi} \leq u^{\varphi}+u^{\psi}$.

(iii) If a given function $\widetilde{G}: \mathbb{R}^{N} \times \mathbb{S}(N) \mapsto \mathbb{R}$ is dominated by $G$, i.e.

$$
\widetilde{G}(p, X)-\widetilde{G}\left(p^{\prime}, X^{\prime}\right) \leq G\left(p-p^{\prime}, X-X^{\prime}\right), \text { for } p, p^{\prime} \in \mathbb{R}, X, X^{\prime} \in \mathbb{S}(N),
$$

then for each $\varphi \in C\left(\mathbb{R}^{N}\right)$ satisfying polynomial growth condition, there exists a unique $\widetilde{G}$-solution $\tilde{u}^{\varphi}(t, x)$ on $[0, \infty) \times \mathbb{R}^{N}$ with initial condition $\left.\tilde{u}^{\varphi}\right|_{t=0}=\varphi$ (see the next section for the proof of existence), i.e.,

$$
\partial_{t} \tilde{u}^{\varphi}-\widetilde{G}\left(D \tilde{u}^{\varphi}, D^{2} \tilde{u}^{\varphi}\right)=0,\left.\quad \tilde{u}^{\varphi}\right|_{t=0}=\varphi .
$$

Moreover

$$
\tilde{u}^{\varphi}(t, x)-\tilde{u}^{\psi}(t, x) \leq u^{\varphi-\psi}(t, x), \text { for } t \geq 0, x \in \mathbb{R}^{N} .
$$

Consequently, the following comparison holds: $\psi \geq \varphi$ implies $\tilde{u}^{\psi} \geq \tilde{u}^{\varphi}$.

Proof. By the above corollaries, it is easy to obtain the results. 


\section{$\S 3$ Perron's Method and Existence}

The combination of Perron's method and viscosity solutions was introduced by H. Ishii [64]. For the convenience of readers, we interpret the proof provided in Crandall, Ishii and Lions [30] into its parabolic situation.

We consider the following parabolic PDE:

$$
\left\{\begin{array}{l}
\partial_{t} u-G\left(t, x, u, D u, D^{2} u\right)=0 \text { on }(0, \infty) \times \mathbb{R}^{N} \\
u(0, x)=\varphi(x) \text { for } x \in \mathbb{R}^{N}
\end{array}\right.
$$

where $G:[0, \infty) \times \mathbb{R}^{N} \times \mathbb{R} \times \mathbb{R}^{N} \times \mathbb{S}(N) \rightarrow \mathbb{R}, \varphi \in C\left(\mathbb{R}^{N}\right)$.

To discuss Perron's method, we will use the following notations: if $u: \mathcal{O} \rightarrow$ $[-\infty, \infty]$ where $\mathcal{O} \subset[0, \infty) \times \mathbb{R}^{N}$, then

$$
\left\{\begin{array}{l}
u^{*}(t, x)=\lim _{r \downarrow 0} \sup \left\{u(s, y):(s, y) \in \mathcal{O} \text { and } \sqrt{|s-t|+|y-x|^{2}} \leq r\right\} \\
u_{*}(t, x)=\lim _{r \downarrow 0} \inf \left\{u(s, y):(s, y) \in \mathcal{O} \text { and } \sqrt{|s-t|+|y-x|^{2}} \leq r\right\} .
\end{array}\right.
$$

One calls $u^{*}$ the upper semicontinuous envelope of $u$; it is the smallest upper semicontinuous function (with values in $[-\infty, \infty]$ ) satisfying $u \leq u^{*}$. Similarly, $u_{*}$ is the lower semicontinuous envelope of $u$.

Theorem 3.1 (Perron's Method) Let comparison hold for (3.14), i.e., if $w$ is a viscosity subsolution of (3.14) and $v$ is a viscosity supersolution of (3.14), then $w \leq v$. Suppose also that there is a viscosity subsolution $\underline{u}$ and a viscosity supersolution $\bar{u}$ of (3.14) that satisfy the condition $\underline{u}_{*}(0, x)=\bar{u}^{*}(0, x)=\varphi(x)$ for $x \in \mathbb{R}^{N}$. Then

$W(t, x)=\sup \{w(t, x): \underline{u} \leq w \leq \bar{u}$ and $w$ is a viscosity subsolution of (3.14) $\}$

is a viscosity solution of (3.14).

The proof consists of two lemmas. For the proof of the following two lemmas, we also see [1]. The first one is

Lemma 3.2 Let $\mathcal{F}$ be a family of viscosity subsolution of 3.14 on $(0, \infty) \times \mathbb{R}^{N}$. Let $w(t, x)=\sup \{u(t, x): u \in \mathcal{F}\}$ and assume that $w^{*}(t, x)<\infty$ for $(t, x) \in$ $(0, \infty) \times \mathbb{R}^{N}$. Then $w^{*}$ is a viscosity subsolution of (3.14) on $(0, \infty) \times \mathbb{R}^{N}$.

Proof. Let $(t, x) \in(0, \infty) \times \mathbb{R}^{N}$ and consider a sequence $s_{n}, y_{n}, u_{n} \in \mathcal{F}$ such that $\lim _{n \rightarrow \infty}\left(s_{n}, y_{n}, u_{n}\left(s_{n}, y_{n}\right)\right)=\left(t, x, w^{*}(t, x)\right)$. There exists $r>0$ such that $N_{r}=\left\{(s, y) \in(0, \infty) \times \mathbb{R}^{N}: \sqrt{|s-t|+|y-x|^{2}} \leq r\right\}$ is compact. For $\phi \in C^{2}$ such that $\phi(t, x)=w^{*}(t, x)$ and $w^{*}<\phi$ on $(0, \infty) \times \mathbb{R}^{N} \backslash(t, x)$, let $\left(t_{n}, x_{n}\right)$ be a maximum point of $u_{n}-\phi$ over $N_{r}$, hence $u_{n}(s, y) \leq u_{n}\left(t_{n}, x_{n}\right)+\phi(s, y)-\phi\left(t_{n}, x_{n}\right)$ for $(s, y) \in N_{r}$. Suppose that (passing to a subsequence if necessary) $\left(t_{n}, x_{n}\right) \rightarrow$ $(\bar{t}, \bar{x})$ as $n \rightarrow \infty$. Putting $(s, y)=\left(s_{n}, y_{n}\right)$ in the above inequality and taking the limit inferior as $n \rightarrow \infty$, we obtain

$$
\begin{aligned}
w^{*}(t, x) & \leq \liminf _{n \rightarrow \infty} u_{n}\left(t_{n}, x_{n}\right)+\phi(t, x)-\phi(\bar{t}, \bar{x}) \\
& \leq w^{*}(\bar{t}, \bar{x})+\phi(t, x)-\phi(\bar{t}, \bar{x}) .
\end{aligned}
$$


From the above inequalities and the assumption on $\phi$, we get $\lim _{n \rightarrow \infty}\left(t_{n}, x_{n}, u_{n}\left(t_{n}, x_{n}\right)\right)=$ $\left(t, x, w^{*}(t, x)\right)$ (without passing to a subsequence). Since $u_{n}$ is a viscosity subsolution of (3.14), by definition we have

$$
\partial_{t} \phi\left(t_{n}, x_{n}\right)-G\left(t_{n}, x_{n}, u_{n}\left(t_{n}, x_{n}\right), D \phi\left(t_{n}, x_{n}\right), D^{2} \phi\left(t_{n}, x_{n}\right)\right) \leq 0 .
$$

Letting $n \rightarrow \infty$, we conclude that

$$
\partial_{t} \phi(t, x)-G\left(t, x, w^{*}(t, x), D \phi(t, x), D^{2} \phi(t, x)\right) \leq 0 .
$$

Thus $w^{*}$ is a viscosity subsolution of (3.14) by definition.

The second step in the proof of Theorem 3.1 is a simple "bump" construction that we now describe. Suppose that $u$ is a viscosity subsolution of (3.14) on $(0, \infty) \times \mathbb{R}^{N}$ and $u_{*}$ is not a viscosity supersolution of (3.14), so that there is $(t, x) \in(0, \infty) \times \mathbb{R}^{N}$ and $\phi \in C^{2}$ with $u_{*}(t, x)=\phi(t, x), u_{*}>\phi$ on $(0, \infty) \times$ $\mathbb{R}^{N} \backslash(t, x)$ and

$$
\partial_{t} \phi(t, x)-G\left(t, x, \phi(t, x), D \phi(t, x), D^{2} \phi(t, x)\right)<0 .
$$

The continuity of $G$ provides $r, \delta_{1}>0$ such that $N_{r}=\left\{(s, y): \sqrt{|s-t|+|y-x|^{2}} \leq\right.$ $r\}$ is compact and

$$
\partial_{t} \phi-G\left(s, y, \phi+\delta, D \phi, D^{2} \phi\right) \leq 0
$$

for all $s, y, \delta \in N_{r} \times\left[0, \delta_{1}\right]$. Lastly, we obtain $\delta_{2}>0$ for which $u_{*}>\phi+\delta_{2}$ on $\partial N_{r}$. Setting $\delta_{0}=\min \left(\delta_{1}, \delta_{2}\right)>0$, we define

$$
U= \begin{cases}\max \left(u, \phi+\delta_{0}\right) & \text { on } N_{r} \\ u & \text { elsewhere. }\end{cases}
$$

By the above inequalities and Lemma 3.2 , it is easy to check that $U$ is a viscosity subsolution of (3.14) on $(0, \infty) \times \mathbb{R}^{N}$. Obviously, $U \geq u$. Finally, observe that $U_{*}(t, x) \geq \max \left(u_{*}(t, x), \phi(t, x)+\delta_{0}\right)>u_{*}(t, x)$; hence there exists $(s, y)$ such that $U(s, y)>u(s, y)$. We summarize the above discussion as the following lemma.

Lemma 3.3 Let $u$ be a viscosity subsolution of (3.14) on $(0, \infty) \times \mathbb{R}^{N}$. If $u_{*}$ fails to be a viscosity supersolution at some point $(s, z)$, then for any small $\kappa>0$ there is a viscosity subsolution $U_{\kappa}$ of (3.14) on $(0, \infty) \times \mathbb{R}^{N}$ satisfying

$$
\left\{\begin{array}{l}
U_{\kappa}(t, x) \geq u(t, x) \text { and } \sup \left(U_{\kappa}-u\right)>0, \\
U_{\kappa}(t, x)=u(t, x) \text { for } \sqrt{|t-s|+|x-z|^{2}} \geq \kappa .
\end{array}\right.
$$

Proof of Theorem 3.1. With the notation of the theorem observe that $\underline{u}_{*} \leq$ $W_{*} \leq W \leq W^{*} \leq \bar{u}^{*}$ and, in particular, $W_{*}(0, x)=W(0, x)=W^{*}(0, x)=\varphi(x)$ for $x \in \mathbb{R}^{N}$. By lemma 3.2. $W^{*}$ is a viscosity subsolution of (3.14) and hence, by comparison, $W^{*} \leq \bar{u}$. It then follows from the definition of $W$ that $W=W^{*}$ (so $W$ is a viscosity subsolution). If $W_{*}$ fails to be a viscosity supersolution at some point $(s, z) \in(0, \infty) \times \mathbb{R}^{N}$, let $W_{\kappa}$ be provided by Lemma 3.3. Clearly $\underline{u} \leq W_{\kappa}$ and $W_{\kappa}(0, x)=\varphi(x)$ for sufficiently small $\kappa$. By comparison, $W_{\kappa} \leq \bar{u}$ 
and since $W$ is the maximal viscosity subsolution between $\underline{u}$ and $\bar{u}$, we arrive at the contradiction $W_{\kappa} \leq W$. Hence $W_{*}$ is a viscosity supersolution of (3.14) and then, by comparison for (3.14), $W^{*}=W \leq W_{*}$, showing that $W$ is continuous and is a viscosity solution of (3.14). The proof is complete.

Let $G: \mathbb{R}^{N} \times \mathbb{S}(N) \rightarrow \mathbb{R}$ be a given continuous sublinear function monotonic in $A \in \mathbb{S}(N)$. We now consider the existence of viscosity solution of the following $G$-equation:

$$
\partial_{t} u-G\left(D u, D^{2} u\right)=0, \quad u(0, x)=\varphi(x) .
$$

Case 1: If $\varphi \in C_{b}^{2}\left(\mathbb{R}^{N}\right)$, then $\underline{u}(t, x)=\underline{M} t+\varphi(x)$ and $\bar{u}(t, x)=\bar{M} t+\varphi(x)$ are respectively the classical subsolution and supersolution of (3.16), where $\underline{M}=$ $\inf _{x \in \mathbb{R}^{N}} G\left(D \varphi(x), D^{2} \varphi(x)\right)$ and $\bar{M}=\sup _{x \in \mathbb{R}^{N}} G\left(D \varphi(x), D^{2} \varphi(x)\right)$. Obviously, $\underline{u}$ and $\bar{u}$ satisfy all the conditions in Theorem 3.1. By Theorem 2.6 we know the comparison holds for (3.16). Thus by Theorem 3.1, we obtain that $G$-equation (3.16) has a viscosity solution.

Case 2: If $\varphi \in C_{b}\left(\mathbb{R}^{N}\right)$ with $\lim _{|x| \rightarrow \infty} \varphi(x)=0$, then we can choose a sequence $\varphi_{n} \in C_{b}^{2}\left(\mathbb{R}^{N}\right)$ which uniformly converge to $\varphi$. For $\varphi_{n}$, by Case 1 , there exists a viscosity solution $u^{\varphi_{n}}$. By comparison theorem, it is easy to show that $u^{\varphi_{n}}$ is uniformly convergent, the limit denoted by $u$. Similar to the proof of Lemma 3.2, it is easy to prove that $u$ is a viscosity solution of $G$-equation (3.16) with initial condition $\varphi$.

Case 3: If $\varphi \in C\left(\mathbb{R}^{N}\right)$ with polynomial growth, then we can choose a large $l>0$ such that $\tilde{\varphi}(x)=\varphi(x) \xi^{-1}(x)$ satisfies the condition in Case 2, where $\xi(x)=\left(1+|x|^{2}\right)^{l / 2}$. It is easy to check that $u$ is a viscosity solution of $G$ equation (3.16) if and only if $\tilde{u}(t, x)=u(t, x) \xi^{-1}(x)$ is a viscosity solution of the following PDE:

$$
\partial_{t} \tilde{u}-\tilde{G}\left(x, \tilde{u}, D \tilde{u}, D^{2} \tilde{u}\right)=0, \tilde{u}(0, x)=\tilde{\varphi},
$$

where $\tilde{G}(x, v, p, X)=G(p+v \eta(x), X+p \otimes \eta(x)+\eta(x) \otimes p+v \kappa(x))$. Here

$$
\begin{aligned}
& \eta(x):=\xi^{-1}(x) D \xi(x)=l\left(1+|x|^{2}\right)^{-1} x, \\
& \kappa(x):=\xi^{-1}(x) D^{2} \xi(x)=l\left(1+|x|^{2}\right)^{-1} I+l(l-2)\left(1+|x|^{2}\right)^{-2} x \otimes x .
\end{aligned}
$$

Similar to the above discussion, we obtain that there exists a viscosity solution of (3.17) with initial condition $\tilde{\varphi}$. Thus there exists a viscosity solution of $G$ equation (3.16).

We summarize the above discussions as a theorem.

Theorem 3.4 Let $\varphi \in C\left(\mathbb{R}^{N}\right)$ with polynomial growth. Then there exists a viscosity solution of $G$-equation (3.16) with initial condition $\varphi$.

Theorem 3.5 Let the function $G$ be given as in the previous theorem and let $\tilde{G}(t, x, p, X):[0, \infty] \times \mathbb{R}^{N} \times \mathbb{R}^{N} \times \mathbb{S}(N) \mapsto \mathbb{R}$ be a given function satisfying condition $(G)$ in Theorem 2.3. We assume that $\tilde{G}$ is dominated by $G$ in the sense of

$$
\begin{aligned}
\tilde{G}(t, x, p, X)-\tilde{G}\left(t, x, p^{\prime}, X^{\prime}\right) \leq & G\left(p-p^{\prime}, X-X^{\prime}\right), \\
& \text { for each } t, x, p, p^{\prime} \text { and } X, X^{\prime} .
\end{aligned}
$$


Then for each given $\varphi \in C\left(\mathbb{R}^{N}\right)$ with polynomial growth condition the viscosity solution of $\partial_{t} \tilde{u}-\tilde{G}\left(t, x, D \tilde{u}, D^{2} \tilde{u}\right)=0$ with $\left.\tilde{u}\right|_{t=0}=\varphi$ exists and is unique. Moreover the comparison property also holds.

Proof. It is easy to check that a $G$-solution with $\left.u\right|_{t=0}=\varphi$ is a $\tilde{G}$-supersolution. Similarly denoting $G_{*}(p, X):=-G(-p,-X)$, a $G_{*}$-solution with $\left.u\right|_{t=0}=\varphi$ is a $\tilde{G}$-subsolution.

We now prove that comparison holds for $\tilde{G}$-solutions. Let $u_{1}$ be a $\tilde{G}$-supersolution and $u_{2}$-be a $\tilde{G}$-subsolution with $\left.u_{1}\right|_{t=0}=\varphi_{1} \in \mathrm{SC}\left([0, T) \times \mathbb{R}^{N}\right),\left.u_{2}\right|_{t=0}=$ $\varphi_{2} \in \operatorname{PSC}\left([0, T) \times \mathbb{R}^{N}\right)$, then by the above domination theorem we can obtain $u_{1}-u_{2} \leq \hat{u}$ where $\hat{u}$ is a $G$-supersolution with $\left.\hat{u}\right|_{t=0}=\varphi_{1}-\varphi_{2}$. On the other hand it is easy to prove that, in the case when $\varphi_{1} \leq \varphi_{2}, \hat{u}=$ $\left(\varphi_{1}(x)-\varphi_{2}(x)\right) \mathbf{1}_{\{0\}}(t)$ such type of $G$-supersolution. Consequently we have $u_{1} \leq u_{2}$. This implies that the comparison holds for $\tilde{G}$-equations. We then can apply Theorem 3.1 to prove that $\tilde{G}$-solution $u$ with $\left.u\right|_{t=0}=\varphi$ exists.

\section{$\S 4$ Krylov's Regularity Estimate for Parabolic PDE}

The proof of our new central limit theorem is based on a powerful $C^{1+\alpha / 2,2+\alpha_{-}}$ regularity estimates for fully nonlinear parabolic PDE obtained in Krylov [76]. A more recent result of Wang [119] (the version for elliptic PDE was initially introduced in Cabre and Caffarelli [17]), using viscosity solution arguments, can also be applied.

For simplicity, we only consider the following type of PDE:

$$
\partial_{t} u+G\left(D^{2} u, D u, u\right)=0, \quad u(T, x)=\varphi(x),
$$

where $G: \mathbb{S}(d) \times \mathbb{R}^{d} \times \mathbb{R} \rightarrow \mathbb{R}$ is a given function and $\varphi \in C_{b}\left(\mathbb{R}^{d}\right)$.

Following Krylov 76], we fix constants $K \geq \varepsilon>0, T>0$ and set $Q=(0, T) \times$ $\mathbb{R}^{d}$. Now we give the definition of $\mathcal{G}(\varepsilon, K, Q)$ and $\overline{\mathcal{G}}(\varepsilon, K, Q)$.

The following definition is according to Definition 5.5.1 in Krylov [76].

Definition 4.1 Let $G: \mathbb{S}(d) \times \mathbb{R}^{d} \times \mathbb{R} \rightarrow \mathbb{R}$ be given, written it as $G\left(u_{i j}, u_{i}, u\right)$, $i, j=1, \ldots, d$. We denote $G \in \mathcal{G}(\varepsilon, K, Q)$ if $G$ is twice continuously differentiable with respect to $\left(u_{i j}, u_{i}, u\right)$ and, for each real-valued $u_{i j}=u_{j i}, \tilde{u}_{i j}=\tilde{u}_{j i}$, $u_{i}, \tilde{u}_{i}, u, \tilde{u}$ and $\lambda^{i}$, the following inequalities hold:

$$
\begin{gathered}
\varepsilon|\lambda|^{2} \leq \sum_{i, j} \lambda^{i} \lambda^{j} \partial_{u_{i j}} G \leq K|\lambda|^{2}, \\
\left|G-\sum_{i, j} u_{i j} \partial_{u_{i j}} G\right| \leq M_{1}^{G}(u)\left(1+\sum_{i}\left|u_{i}\right|^{2}\right), \\
\left|\partial_{u} G\right|+\left(1+\sum_{i}\left|u_{i}\right|\right) \sum_{i}\left|\partial_{u_{i}} G\right| \leq M_{1}^{G}(u)\left(1+\sum_{i}\left|u_{i}\right|^{2}+\sum_{i, j}\left|u_{i j}\right|\right),
\end{gathered}
$$




$$
\begin{aligned}
{\left[M_{2}^{G}\left(u, u_{k}\right)\right]^{-1} G_{(\eta)(\eta)} \leq } & \sum_{i, j}\left|\tilde{u}_{i j}\right|\left[\sum_{i}\left|\tilde{u}_{i}\right|+\left(1+\sum_{i, j}\left|u_{i j}\right|\right)|\tilde{u}|\right] \\
& +\sum_{i}\left|\tilde{u}_{i}\right|^{2}\left(1+\sum_{i, j}\left|u_{i j}\right|\right)+\left(1+\sum_{i, j}\left|u_{i j}\right|^{3}\right)|\tilde{u}|^{2}
\end{aligned}
$$

where the arguments $\left(u_{i j}, u_{i}, u\right)$ of $G$ and its derivatives are omitted, $\eta=\left(\tilde{u}_{i j}, \tilde{u}_{i}, \tilde{u}\right)$, and

$$
\begin{aligned}
G_{(\eta)(\eta)}:= & \sum_{i, j, r, s} \tilde{u}_{i j} \tilde{u}_{r s} \partial_{u_{i j} u_{r s}}^{2} G+2 \sum_{i, j, r} \tilde{u}_{i j} \tilde{u}_{r} \partial_{u_{i j} u_{r}}^{2} G+2 \sum_{i, j} \tilde{u}_{i j} \tilde{u} \partial_{u_{i j} u}^{2} G \\
& +\sum_{i, j} \tilde{u}_{i} \tilde{u}_{j} \partial_{u_{i} u_{j}}^{2} G+2 \sum_{i} \tilde{u}_{i} \tilde{u} \partial_{u_{i} u}^{2} G+|\tilde{u}|^{2} \partial_{u u}^{2} G
\end{aligned}
$$

$M_{1}^{G}(u)$ and $M_{2}^{G}\left(u, u_{k}\right)$ are some continuous functions which grow with $|u|$ and $u_{k} u_{k}$ and $M_{2}^{G} \geq 1$.

Remark 4.2 Let $\varepsilon I \leq A=\left(a_{i j}\right) \leq K I$. It is easy to check that

$$
G\left(u_{i j}, u_{i}, u\right)=\sum_{i, j} a_{i j} u_{i j}+\sum_{i} b_{i} u_{i}+c u
$$

belongs to $\mathcal{G}(\varepsilon, K, Q)$.

The following definition is Definition 6.1.1 in Krylov [76].

Definition 4.3 Let a function $G=G\left(u_{i j}, u_{i}, u\right): \mathbb{S}(d) \times \mathbb{R}^{d} \times \mathbb{R} \rightarrow \mathbb{R}$ be given. We write $G \in \overline{\mathcal{G}}(\varepsilon, K, Q)$ if there exists a sequence $G_{n} \in \mathcal{G}(\varepsilon, K, Q)$ converging to $G$ as $n \rightarrow \infty$ at every point $\left(u_{i j}, u_{i}, u\right) \in \mathbb{S}(d) \times \mathbb{R}^{d} \times \mathbb{R}$ such that

(i) $M_{i}^{G_{1}}=M_{i}^{G_{2}}=\cdots=: M_{i}^{G}, i=1,2$;

(ii) for each $n=1,2, \ldots$, the function $G_{n}$ is infinitely differentiable with respect to $\left(u_{i j}, u_{i}, u\right)$

(iii) there exist constants $\delta_{0}=: \delta_{0}^{G}>0$ and $M_{0}=: M_{0}^{G}>0$ such that

$$
G_{n}\left(u_{i j}, 0,-M_{0}\right) \geq \delta_{0}, G_{n}\left(-u_{i j}, 0, M_{0}\right) \leq-\delta_{0}
$$

for each $n \geq 1$ and symmetric nonnegative matrices $\left(u_{i j}\right)$.

The following theorem is Theorem 6.4.3 in Krylov [76], which plays important role in our proof of central limit theorem.

Theorem 4.4 Suppose that $G \in \overline{\mathcal{G}}(\varepsilon, K, Q)$ and $\varphi \in C_{b}\left(\mathbb{R}^{d}\right)$ with $\sup _{x \in \mathbb{R}^{d}}|\varphi(x)| \leq$ $M_{0}^{G}$. Then PDE (4.18) has a solution u possessing the following properties:

(i) $u \in C\left([0, T] \times \mathbb{R}^{d}\right),|u| \leq M_{0}^{G}$ on $Q$; 
(ii) there exists a constant $\alpha \in(0,1)$ only depending on $d, K, \varepsilon$ such that for each $\kappa>0$,

$$
\|u\|_{C^{1+\alpha / 2,2+\alpha}\left([0, T-\kappa] \times \mathbb{R}^{d}\right)}<\infty .
$$

Now we consider the $G$-equation. Let $G: \mathbb{R}^{d} \times \mathbb{S}(d) \rightarrow \mathbb{R}$ be a given continuous sublinear function monotonic in $A \in \mathbb{S}(d)$. Then there exists a bounded, convex and closed subset $\Sigma \subset \mathbb{R}^{d} \times \mathbb{S}_{+}(d)$ such that

$$
G(p, A)=\sup _{(q, B) \in \Sigma}\left[\frac{1}{2} \operatorname{tr}[A B]+\langle p, q\rangle\right] \quad \text { for }(p, A) \in \mathbb{R}^{d} \times \mathbb{S}(d) .
$$

The $G$-equation is

$$
\partial_{t} u+G\left(D u, D^{2} u\right)=0, \quad u(T, x)=\varphi(x) .
$$

We set

$$
\tilde{u}(t, x)=e^{t-T} u(t, x) .
$$

It is easy to check that $\tilde{u}$ satisfies the following PDE:

$$
\partial_{t} \tilde{u}+G\left(D \tilde{u}, D^{2} \tilde{u}\right)-\tilde{u}=0, \quad \tilde{u}(T, x)=\varphi(x) .
$$

Suppose that there exists a constant $\varepsilon>0$ such that for each $A, \bar{A} \in \mathbb{S}(d)$ with $A \geq \bar{A}$, we have

$$
G(0, A)-G(0, \bar{A}) \geq \varepsilon \operatorname{tr}[A-\bar{A}] .
$$

Since $G$ is continuous, it is easy to prove that there exists a constant $K>0$ such that for each $A, \bar{A} \in \mathbb{S}(d)$ with $A \geq \bar{A}$, we have

$$
G(0, A)-G(0, \bar{A}) \leq K \operatorname{tr}[A-\bar{A}] .
$$

Thus for each $(q, B) \in \Sigma$, we have

$$
2 \varepsilon I \leq B \leq 2 K I .
$$

By Remark 4.2, it is easy to check that $\tilde{G}\left(u_{i j}, u_{i}, u\right):=G\left(u_{i}, u_{i j}\right)-u \in$ $\overline{\mathcal{G}}(\varepsilon, K, Q)$ and $\delta_{0}^{G}=M_{0}^{G}$ can be any positive constant. By Theorem 4.4 and (4.22), we have the following regularity estimate for $G$-equation (4.21).

Theorem 4.5 Let $G$ satisfy (4.20) and (4.24), $\varphi \in C_{b}\left(\mathbb{R}^{d}\right)$ and let $u$ be a solution of $G$-equation (4.21). Then there exists a constant $\alpha \in(0,1)$ only depending on $d, G, \varepsilon$ such that for each $\kappa>0$,

$$
\|u\|_{C^{1+\alpha / 2,2+\alpha}\left([0, T-\kappa] \times \mathbb{R}^{d}\right)}<\infty .
$$




\section{Bibliography}

[1] Alvarez, O. and Tourin, A. (1996) Viscosity solutions of nonlinear integrodifferential equations. Ann. Inst. H. Poincaré Anal. Non Linéaire, 13(3), $293-317$.

[2] Artzner, Ph., Delbaen, F., Eber, J. M. and Heath, D. (1997) Thinking coherently RISK 10, 86-71.

[3] Artzner, Ph., Delbaen, F., Eber, J. M. and Heath, D. (1999) Coherent Measures of Risk, Mathematical Finance 9, 203-228.

[4] Atlan,M. (2006) Localizing volatilities, in arXiv:math/0604316v1 [math.PR].

[5] Avellaneda, M., Levy, A. and Paras, A. (1995) Pricing and hedging derivative securities in markets with uncertain volatilities. Appl. Math. Finance 2 73-88.

[6] Bachelier, L. (1990) Théorie de la spéculaton. Annales Scientifiques de l'École Normale Supérieure, 17, 21-86

[7] Barenblatt, G. I. (1979) Similarity, Self-Similarity and Intermediate Asymptotics, Consultants Bureau, New York.

[8] Barles, G. (1994) Solutions de viscosité des équations de Hamilton-Jacobi. Collection "Math ématiques et Applications" de la SMAI, no.17, SpringerVerlag.

[9] Barrieu, P. and El Karoui, N. (2004) Pricing, hedging and optimally designing derivatives via minimization of risk measures, Preprint, in Rene Carmona (Eds.), Volume on Indifference Pricing, Princeton University Press (in press).

[10] Bensoussan, A. (1981) Lecture on stochastic control, Lecture Notes in Mathematics, Vol.972, Springer-Verlag.

[11] Bensoussan, A. (1982) Stochastic Control by Functional Analysis Methods, North-Holland. 
[12] Bismut, J.M. (1973) Conjugate convex functions in optimal stochastic control, J.Math. Anal. Apl. 44, pp.384-404.

[13] Bismut, J.M. (1978) Contrôle des systemes linéaires quadratiques : applications de l'integrale stochastique, Sémin. Proba. XII., Lect. Notes in Mathematics, 649, pp.180-264, Springer.

[14] Briand, Ph., Coquet, F., Hu, Y., Mémin J. and Peng, S. (2000) A converse comparison theorem for BSDEs and related properties of g-expectations, Electron. Comm. Probab, 5, 101-117.

[15] Bogachev, V.I.: Gaussian Measures. Amer. Math. Soc., Mathematical Surveys and Monographs, Vol 62 (1998)

[16] Bouleau,N., Hirsch,F. (1991) Dirichlet forms and analysis on Wiener space. De Gruyter Studies in Math.

[17] Cabre, X. and Caffarelli, L. A. (1997) Fully Nonlinear Elliptic Partial Differential Equations, American Math. Society.

[18] Chen, Z. (1998) A property of backward stochastic differential equations, C.R. Acad. Sci. Paris Sér.I 326(4), 483-488.

[19] Chen, Z. and Epstein, L. (2002) Ambiguity, risk and asset returns in continuous time, Econometrica, 70(4), 1403-1443.

[20] Chen, Z., Kulperger, R. and Jiang L. (2003) Jensen's inequality for gexpectation: part 1, C. R. Acad. Sci. Paris, Sér.I 337, 725-730.

[21] Chen, Z. and Peng, S. (1998) A nonlinear Doob-Meyer type decomposition and its application. SUT Journal of Mathematics (Japan), 34(2), 197-208.

[22] Chen, Z. and Peng, S. (2000) A general downcrossing inequality for gmartingales, Statist. Probab. Lett. 46(2), 169-175.

[23] Cheridito, P., Soner, H.M., Touzi, N. and Victoir, N. (2007) Second order backward stochastic differential equations and fully non-linear parabolic PDEs, Communications on Pure and Applied Mathematics, 60 (7), 10811110.

[24] Choquet, G. (1953) Theory of capacities, Annales de Institut Fourier, 5, 131-295.

[25] Chung, K.L. and Williams, R. (1990) Introduction to Stochastic Integration, 2nd Edition, Birkhäuser.

[26] Coquet, F., Hu, Y., Mémin, J. and Peng, S. (2001) A general converse comparison theorem for Backward stochastic differential equations, C.R.Acad. Sci. Paris, t.333, Serie I, 577-581. 
[27] Coquet, F., Hu, Y., Mémin J. and Peng, S. (2002) Filtration-consistent nonlinear expectations and related g-expectations, Probab. Theory Relat. Fields, 123, 1-27.

[28] Crandall, M.G. and Lions, P.L., Condition d'unicité pour les solutions generalisées des équations de Hamilton-Jacobi du premier ordre, C. R. Acad. Sci. Paris Sér. I Math. 292 (1981), 183-186.

[29] Crandall, M. G. and Lions, P. L. (1983) Viscosity solutions of HamiltonJacobi equations, Trans. Amer. Math. Soc. 277, 1-42.

[30] Crandall, M. G., Ishii, H., and Lions, P. L. (1992) User's guide to viscosity solutions of second order partial differential equations, Bulletin Of The American Mathematical Society, 27(1), 1-67.

[31] Daniell, P.J. (1918) A general form of integral. The Annals of Mathematics, 19, 279-294.

[32] Dellacherie,C. (1972) Capacités et Processus Stochastiques. Springer Verlag.

[33] Dellacherie, C. and Meyer, P.A. (1978 and 1982) Probabilities and Potential A and B, North-Holland.

[34] Delbaen, F. (1992) Representing martingale measures when asset prices are continuous and bounded. Math. Finance 2, No.2, 107-130.

[35] Delbaen, F. (2002) Coherent Risk Measures (Lectures given at the Cattedra Galileiana at the Scuola Normale di Pisa, March 2000), Published by the Scuola Normale di Pisa.

[36] Delbaen, F., Rosazza, G. E. and Peng S. (2005) m-Stable sets, risk measures via g-expectations, Preprint.

[37] Denis, L., Hu, M. and Peng, S. (2008) Function spaces and capacity related to a sublinear expectation: application to G-Brownian motion paths, see arXiv:0802.1240v1 [math.PR] 9 Feb, 2008.

[38] Denis, L. and Martini, C. (2006) A theoretical framework for the pricing of contingent claims in the presence of model uncertainty, The Ann. of Appl. Probability 16(2), 827-852.

[39] Denneberg, D. (1994) Non-Additive Measure and Integral, Kluwer.

[40] Doob, J. L. (1984) Classical Potential Theory and its Probabilistic Counterpart. Springer, NY.

[41] Dudley, R. M. (1989) Real analysis and Probability. Wadsworth, Brooks\& Cole, Pacific Grove, CA. 
[42] Einstein, A. (1905) On the movement of small particles suspended in a stationary liquid demanded by the molecular-kinetic theory of heat. Engl. trans. in Investigations on the Theory of the Brownian Movement. Repr. Dover, NY 1956.

[43] El Karoui, N. and Quenez, M.C. (1995) Dynamic programming and pricing of contingent claims in incomplete market. SIAM J.of Control and Optimization, 33(1).

[44] El Karoui, N., Peng, S. and Quenez, M.C. (1997) Backward stochastic differential equation in finance, Mathematical Finance 7(1): 1-71.

[45] Evans, L. C. (1978) A convergence theorem for solutions of nonlinear second order elliptic equations, Indiana Univ. Math. J. 27, 875-887.

[46] Evans, L. C. (1980) On solving certain nonlinear differential equations by accretive operator methods, Israel J. Math. 36, 225-247.

[47] Feller, W. $(1968,1971)$ An Introduction to Probability Theory and its Applications, 1 (3rd ed.);2 (2nd ed.). Wiley, NY.

[48] Feyel, D. and de La Pradelle, A. (1989) Espaces de Sobolev gaussiens. Ann. Inst. Fourier 39 875-908.

[49] Fleming, W.H. and Soner, H.M. (1992) Controlled Markov Processes and Viscosity Solutions. Springer-Verleg, New York.

[50] Föllmer,H., Schied, A. (2002) Convex measures of risk and trading constraints . Finance and Stochastics 6 (4), 429-447.

[51] Föllmer, H. and Schied, A. (2004) Statistic Finance, An introduction in discrete time (2nd Edition), Walter de Gruyter.

[52] Frittelli, M. and Rossaza Gianin, E. (2002) Putting order in risk measures, Journal of Banking and Finance, 26(7) 1473-1486.

[53] Frittelli, M. and Rossaza Gianin, E. (2004) Dynamic convex risk measures, Risk Measures for the 21st Century, J. Wiley. 227-247.

[54] Gao, F.Q. (2009) Pathwise properties and homeomorphic flows for stochastic differential equations driven by $G$-Brownian motion Stochastic Processes and their Applications Volume 119, Issue 10, Pages 3356-3382.

[55] Gao Fuqing and Jiang Hui (2009) Large Deviations For Stochastic Differential Equations Driven By G-Brownian Motion, preprint.

[56] Gao, F.Q. and Xu M.Z. (2009) Large Deviations And Moderate Deviations for Independent Random Variables under Sublinear Expectation, Preprint.

[57] He, S.W., Wang, J.G. and Yan J. A. (1992) Semimartingale Theory and Stochastic Calculus, CRC Press, Beijing. 
[58] Hu,M. and Peng,S. (2009) On Representation Theorem of $G$-Expectations and Paths of $G$-Brownian Motion, Acta Mathematicae Applicatae Sinica, English Series 25(3), 539-546.

[59] Hu,M. and Peng,S. (2009) G-Lévy Processes under Sublinear Expectations, preprint: arXiv:0911.3533v1 [math.PR] 18 Nov 2009.

[60] Hu, Y. (2005) On Jensens inequality for g-expectation and for nonlinear expectation, Archiv der Mathematik, Volume 85, Number 6, 572-580.

[61] Huber,P. J. (1981) Robust Statistics, John Wiley \& Sons.

[62] Huber,P., Strassen,V. (1973) Minimax tests and the Neyman-Pearson Lemma for capacity. The Annals of Statistics, Vol. 1, No. 2 pp 252-263.

[63] Ikeda, N. and Watanabe, S. (1981) Stochastic Differential Equations and Diffusion Processes, North-Holland, Amsterdam.

[64] Ishii, H. (1987) Perron's method for Hamilton-Jacobi equations, Duke Math. J. 55, 369-384.

[65] Itô, K. Differential equations determining a Markoff process, Journ. PanJapan Math. Coll. No. 1077, 1942, In Kiyosi Itô: Selected Papers, Springer, 1987.

[66] Itô, K. and McKean, M. (1965) Diffusion Processes and Their Sample Paths, Springer-Verlag.

[67] Jensen, R. (1988) The maximum principle for viscosity solutions of fully nonlinear second order partial differential equations, Arch. Rat. Mech. Anal. 101, 1-27.

[68] Jia, G. and Peng, S. (2007) A new look at Jensen's inequality for gexpectations: g-convexity and their applications, Preprint.

[69] Jiang, L. (2004) Some results on the uniqueness of generators of backward stochastic differential equations, C. R. Acad. Sci. Paris, Ser. I 338 575580 .

[70] Jiang L. and Chen, Z. (2004) A result on the probability measures dominated by g-expectation, Acta Mathematicae Applicatae Sinica, English Series 20(3) 507-512.

[71] Jiang L. and Chen Z. (2004) On Jensen's inequality for g-expectation, Chin. Ann. Math. 25B(3),401-412.

[72] Kallenberg, O. (2002) Foundations of Modern Probability (2nd edition), Springer-Verlag.

[73] Karatzas, I. and Shreve, S. E., (1988) Brownian Motion and Stochastic Calculus, Springer-Verlag, New York. 
[74] Kolmogorov.A.N. (1956) Foundations of the Theory of Probability. Chelsea, New York, 1956; second edition [Osnovnye poniatiya teorii veroyatnostei]. "Nauka", Moscow, 1974.

[75] Krylov, N.V. (1980) Controlled Diffusion Processes. Springer-Verlag, New York.

[76] Krylov, N.V. (1987) Nonlinear Parabolic and Elliptic Equations of the Second Order, Reidel Publishing Company (Original Russian version by Nauka, Moscow, 1985).

[77] Lévy, P. (1925) Calcul dés Probabilités, Gautier-Villars.

[78] Lévy P. (1965) Processus Stochastiques et Mouvement Brownian, Jacques Gabay, 2ème édition, Gautier-Villars.

[79] Li, X and Peng S., (2009) Stopping times and related Itô's calculus with $G$-Brownian motion, Preprint in arXiv:0910.3871v1 [math.PR].

[80] Lions, P.L. (1982) Some recent results in the optimal control of diffusion processes, Stochastic analysis, Proceedings of the Taniguchi International Symposium on Stochastic Analysis (Katata and Kyoto 1982), Kinokuniya, Tokyo.

[81] Lions, P.L. (1983) Optimal control of diffusion processes and HamiltonJacobi-Bellman equations. Part 1: The dynamic programming principle and applications and Part 2: Viscosity solutions and uniqueness, Comm. Partial Differential Equations 8, 1101-1174 and 1229-1276.

[82] Lyons, T. (1995). Uncertain volatility and the risk free synthesis of derivatives. Applied Mathematical Finance 2, 11 7-133.

[83] Marinacci, M. (1999) Limit laws for non-additive probabilities and their frequentist interpretation, Journal of Economic Theory 84, 145-195

[84] Maccheroni, F. and Marinacci, M. (2005) A strong law of large numbers for capacities. The Annals of Probability Vol. 33, No. 3, 1171-1178.

[85] Nisio, M. (1976) On a nonlinear semigroup attached to optimal stochastic control. Publ. RIMS, Kyoto Univ., 13: 513-537.

[86] Nisio, M. (1976) On stochastic optimal controls and envelope of Markovian semi-groups. Proc. of int. Symp. Kyoto, 297-325.

[87] Øksendal B. (1998) Stochastic Differential Equations, Fifth Edition, Springer.

[88] Pardoux, E., Peng, S. (1990) Adapted solutions of a backward stochastic differential equation, Systems and Control Letters, 14(1): 55-61. 
[89] Peng, S. (1991) Probabilistic Interpretation for Systems of Quasilinear Parabolic Partial Differential Equations, Stochastics, 37, 61-74.

[90] Peng, S. (1992) A generalized dynamic programming principle and Hamilton-Jacobi-Bellman equation. Stochastics and Stochastic Reports, 38(2): 119-134.

[91] Peng S. (1992) A Nonlinear Feynman-Kac Formula and Applications, in Proceedings of Symposium of System Sciences and Control Theory, Chen \& Yong eds., 173-184, World Scientific, Singapore.

[92] Peng, S. (1997) Backward SDE and related g-expectations, in Backward Stochastic Differential Equations, Pitman Research Notes in Math. Series, No.364, El Karoui Mazliak edit. 141-159.

[93] Peng, S. (1997) BSDE and Stochastic Optimizations, Topics in Stochastic Analysis, Yan, J., Peng, S., Fang, S., Wu, L.M. Ch.2, (Chinese vers.), Science Press, Beijing.

[94] Peng, S. (1999) Monotonic limit theorem of BSDE and nonlinear decomposition theorem of Doob-Meyer's type, Prob. Theory Rel. Fields 113(4) 473-499.

[95] Peng, S. (2004) Nonlinear expectation, nonlinear evaluations and risk measurs, in K. Back T. R. Bielecki, C. Hipp, S. Peng, W. Schachermayer, Stochastic Methods in Finance Lectures, 143-217, LNM 1856, SpringerVerlag.

[96] Peng, S. (2004) Filtration consistent nonlinear expectations and evaluations of contingent claims, Acta Mathematicae Applicatae Sinica, English Series 20(2), 1-24.

[97] Peng, S. (2004) Dynamical evaluations, C. R. Acad. Sci. Paris, Ser.I 339 $585-589$.

[98] Peng, S. (2005) Nonlinear expectations and nonlinear Markov chains, Chin. Ann. Math. 26B(2), 159-184.

[99] Peng, S. (2005), Dynamically consistent nonlinear evaluations and expectations, preprint (pdf-file available in arXiv:math.PR/0501415 v1 24 Jan 2005).

[100] Peng, S. (2006) G-Expectation, $G$-Brownian Motion and Related Stochastic Calculus of Itô's type, The Abel Symposium 2005, Abel Symposia 2, Edit. Benth et. al., 541-567, Springer-Verlag, 2006.

[101] Peng, S. (2007) Law of large numbers and central limit theorem under nonlinear expectations, in arXiv:math.PR/0702358v1 13 Feb 2007.

[102] Peng, S. (2007) Lecture Notes: G-Brownian motion and dynamic risk measure under volatility uncertainty, in arXiv:0711.2834v1 [math.PR]. 
[103] Peng, S. (2007) Law of large numbers and central limit theorem under nonlinear expectations, in arXiv:math.PR/0702358v1 13 Feb 2007

[104] Peng, S. (2008) Multi-dimensional G-Brownian motion and related stochastic calculus under $G$-expectation, Stochastic Processes and their Applications, 118(12), 2223-2253.

[105] Peng, S. (2008) A new central limit theorem under sublinear expectations, in arXiv:0803.2656v1 [math.PR].

[106] Peng, S. (2009) Survey on normal distributions, central limit theorem, Brownian motion and the related stochastic calculus under sublinear expectations, Science in China Series A: Mathematics, Volume 52, Number 7, 1391-1411.

[107] Peng, S. and Xu, M. (2006) The Numerical Algorithms and Simulations for BSDEs, Preprint, see arXiv:math.PR/0611864 v1.

[108] Peng, S. and Xu, M. (2005) $g_{\Gamma^{-}}$expectations and the Related Nonlinear Doob-Meyer Decomposition Theorem, preprint.

[109] Rogers, L. C.G. and Williams, D. (2000a/b) Diffusions, Markov Processes, and Martingales, 1 (2nd ed.);2. Cambridge Univ. Press.

[110] Protter, Ph. (1990) Stochastic Integration and Differential Equations, Springer-Verlag.

[111] Revuz, D. and Yor, M. (1999) Continuous Martingales and Brownian Motion, 3rd ed. Springer, Berlin.

[112] Rosazza, G. E. (2006) Some examples of risk measures via g-expectations, preprint, Insurance: Mathematics and Economics, Volume 39, 19-34.

[113] Shiryaev, A.N. (1984) Probability, 2nd Edition, Springer-Verlag.

[114] Soner, M., Touzi, N. and Zhang, J. (2009) Martingale Representation Theorem under $G$-expectation. Preprint.

[115] Song, Y. (2007) A general central limit theorem under Peng's G-normal distribution, Preprint.

[116] Song, Y. (2009) Some properties on $G$-evaluation and its applications to $G$-martingale decomposition, Preprint.

[117] Stroock, D.W., Varadhan, S.R.S. (1979). Multidimensional Diffusion Processes. Springer-Verlag.

[118] Szegö, G. (edits) (2004) Risk Measures for the 21 Centrury, WileyFinance.

[119] Wang, L. (1992) On the regularity of fully nonlinear parabolic equations: II, Comm. Pure Appl. Math. 45, 141-178. 
[120] Walley, P. (1991) Statistical Reasoning with Imprecise Probabilities, Chapman and Hall, London, New York.

[121] Wiener, N. (1923) Differential space. J. Math. PHys. 2, 131-174.

[122] Xu, J. and Zhang, B. Martingale characterization of G-Brownian motion, Stochastic Processes and their Applications 119, 232-248.

[123] Yan, J.-A. (1998) Lecture Note on Measure Theory, Science Press, Beijing (Chinese version).

[124] Yong, J. and Zhou, X. (1999) Stochastic Controls: Hamiltonian Systems and HJB Equations. Springer-Verlag.

[125] Yosida, K. (1980) Functional Analysis, Sixth-Edition, Springer. 


\section{Index of Symbols}

$\mathcal{A}$

$B_{b}(\Omega)$

$B$

$\langle B\rangle$

$C_{b}(\Omega)$

$C_{b, \text { Lip }}\left(\mathbb{R}^{n}\right)$

$C_{l, L i p}\left(\mathbb{R}^{n}\right)$

$\mathbb{E}$

$\hat{\mathbb{E}}$

$\mathcal{H}$

$L^{0}(\Omega)$

$\mathbb{L}_{b}^{p}$

$\mathbb{L}_{c}^{p}$

$M_{G}^{p, 0}(0, T)$

$M_{G}^{p}(0, T)$

$\bar{M}_{G}^{p}(0, T)$

q.s.

$\mathbb{S}(d)$

$\mathbb{S}_{+}(d)$

$\rho$

$(\Omega, \mathcal{H}, \mathbb{E})$

$\underline{\underline{d}}$

$\langle x, y\rangle$

$|x|$

$(A, B)$
Coherent acceptable set 14

Space of bounded functions on $\Omega 91$

G-Brownian motion 36

Quadratic variation process of $B \quad 46$

Space of bounded and continuous functions on $\Omega 91$

Space of bounded and Lipschitz continuous functions on $\mathbb{R}^{n}$

Space of locally Lipschitz functions 2

Sublinear expectation 1

$G$-expectation 39

Space of random variables 1

Space of all $\mathcal{B}(\Omega)$-measurable real functions 91

Completion of $B_{b}(\Omega)$ under norm $\|\cdot\|_{p} \quad 95$

Completion of $C_{b}(\Omega)$ under norm $\|\cdot\|_{p}$

Space of simple processes 42

Completion of $M_{G}^{p, 0}(0, T)$ under norm $\left\{\hat{\mathbb{E}}\left[\int_{0}^{T}\left|\eta_{t}\right|^{p} d t\right]\right\}^{1 / p}$

Completion of $M_{G}^{p, 0}(0, T)$ under norm $\left\{\int_{0}^{T} \hat{\mathbb{E}}\left[\left|\eta_{t}\right|^{p} d t\right]\right\}^{1 / p}$

Quasi-surely 92

Space of $d \times d$ symmetric matrices 18

Space of non-negative $d \times d$ symmetric matrices 22

Coherent risk measure 14

Sublinear expectation space 2

Identically distributed 7

Scalar product of $x, y \in \mathbb{R}^{n}$

Euclidean norm of $x$

Inner product $(A, B):=\operatorname{tr}[A B]$ 


\section{Index}

$G$-Brownian motion, 36

$G$-convex, 75

$G$-distributed, 19

$G$-equation, 19

$G$-expectation, 39

$G$-heat equation, 22

$G$-martingale, 71

$G$-normal distribution, 18

$G$-submartingale, 71

$G$-supermartingale, 71

Banach space, 107

Bochner integral, 43

Cauchy sequence, 107

Cauchy's convergence condition, 107

Central limit theorem with law of large numbers, 27

Central limit theorem with zero-mean, 26

Coherent acceptable set, 14

Coherent risk measure, 14

Complete, 107

Converge in distribution, 9, 111

Converge in law, 9, 111

Converge weakly, 111

Convex acceptable set, 14

Convex expectation, 2

Daniell's integral, 112

Daniell-Stone theorem, 112

Dini's theorem, 108

Distribution, 6

Domination Theorem, 116

Einstein convention, 56, 72

Extension, 108
Finite dimensional distributions, 109

Generalized $G$-Brownian motion, 61

Geometric $G$-Brownian motion, 83

Hahn-Banach extension theorem, 108

Identically distributed, 7

Independent, 9

Independent copy, 11

Kolmogorov's continuity criterion, 110

Kolmogorov's criterion for weak compactness, 111

Kolmogorov's extension theorem, 110

Law of large numbers, 25

Lower semicontinuous envelope, 122

Maximal distribution, 17

Mean-uncertainty, 7

Modification, 110

Mutual variation process, 49

Nonlinear expectation, 2

Nonlinear expectation space, 2

Nonlinear G-Brownian motion, 64

Parabolic subjet, 114

Parabolic superjet, 114

Polar, 92

Product space of sublinear expectation space, 10

Prokhorov's criterion, 111

Quadratic variation process, 46

Quasi-surely, 92

Regular, 93 
Restriction, 108

Robust expectation, 3

Stochastic process, 35

Sublinear expectation, 1

Sublinear expectation space, 2

Sublinearity, 2

Tight, 111

Upper semicontinuous envelope, 122

Variance-uncertainty, 7

Vector lattice, 112

Version, 110

Viscosity solution, 114, 115

Viscosity subsolution, 114

Viscosity supersolution, 114, 115

Weakly relatively compact, 111 